\title{
IntechOpen
}

\section{Vascular Malformations of the Central Nervous System}

Edited by Bora Gürer and Pinar Kuru Bektaşoğlu 



\title{
Vascular Malformations of the Central Nervous System
}

\author{
Edited by Bora Gürer \\ and Pinar Kuru Bektaşoğlu
}



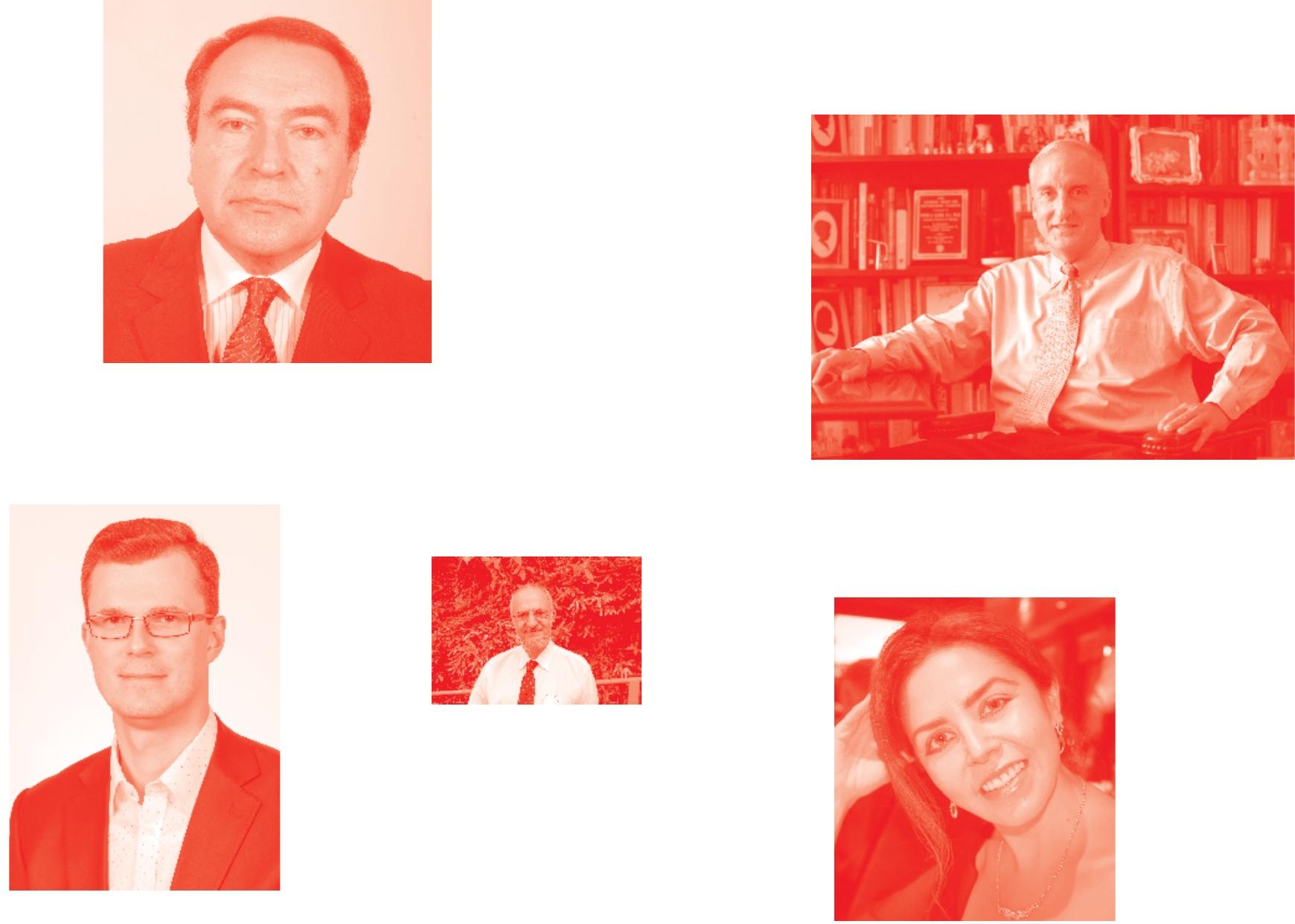

Supporting open minds since 2005
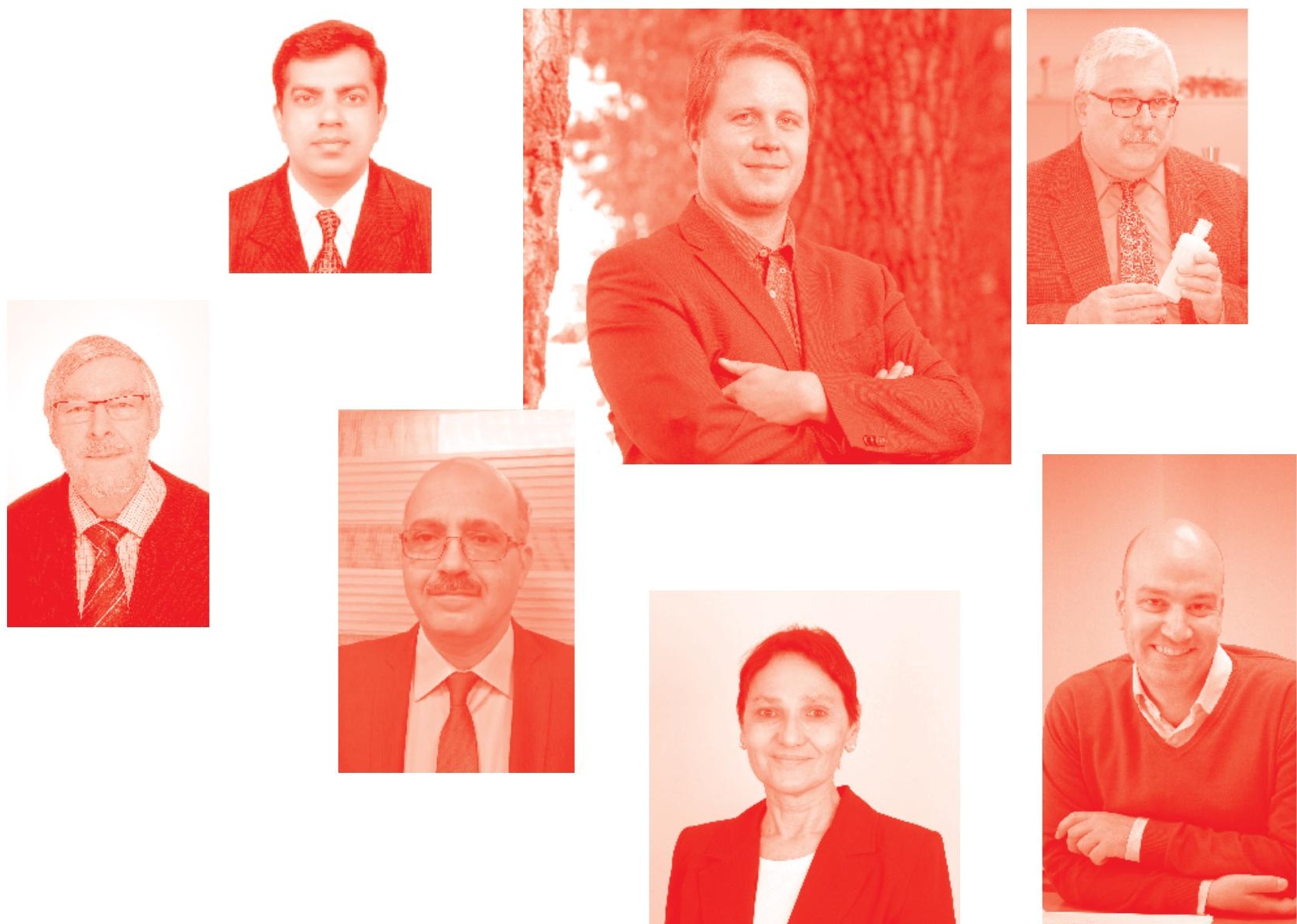
Vascular Malformations of the Central Nervous System

http : //dx. doi. org/10.5772/intechopen. 78160

Edited by Bora Gürer and Pinar Kuru Bektaşoğlu

\section{Contributors}

Nissar Shaikh, Amer Jaradat, Sara Abusini, Nour Tashtush, Abdelwahab Aleshawi, Adel. E. Ahmad Ganaw, Abdulgafoor Tharayil, Moad Ehfeda, Ali Ayyad, Nabil Shalik, Dr Nissar Shaikh, Ibrahim Naemi, Mohamed Alfared, Qazi Zeeshan Ul Haq, Hossam Algallie, Sohel Mohamed Gamal Ahmed, Sujith Madambikattil Prabhakaran, Arshad Husain Chanda, Simi Praveen, Ajithkumar Choran, Sedef Tavukçu Özkan, Themistoklis Papasilekas, Konstantinos Themistoklis, Stefanos Korfias, Damianos Sakas, Saar Golan, Amir Arthur, Nitzan Hirsh, David Kieser, Sherry Soltani, Michael Wyatt, Khoon S Lim, Sandra Kieser, Cagin Senturk

(-) The Editor(s) and the Author(s) 2020

The rights of the editor(s) and the author(s) have been asserted in accordance with the Copyright, Designs and Patents Act 1988. All rights to the book as a whole are reserved by INTECHOPEN LIMITED . The book as a whole (compilation) cannot be reproduced, distributed or used for commercial or non-commercial purposes without INTECHOPEN LIMITED's written permission. Enquiries concerning the use of the book should be directed to INTECHOPEN LIMITED rights and permissions department (permissions@intechopen.com).

Violations are liable to prosecution under the governing Copyright Law

\section{(cc) BY}

Individual chapters of this publication are distributed under the terms of the Creative Commons Attribution 3.0 Unported License which permits commercial use, distribution and reproduction of the individual chapters, provided the original author(s) and source publication are appropriately acknowledged. If so indicated, certain images may not be included under the Creative Commons license. In such cases users will need to obtain permission from the license holder to reproduce the material. More details and guidelines concerning content reuse and adaptation can be found at http : //www . intechopen . com/copyright-policy . html .

\section{Notice}

Statements and opinions expressed in the chapters are these of the individual contributors and not necessarily those of the editors or publisher. No responsibility is accepted for the accuracy of information contained in the published chapters. The publisher assumes no responsibility for any damage or injury to persons or property arising out of the use of any materials, instructions, methods or ideas contained in the book.

First published in London, United Kingdom, 2020 by IntechOpen IntechOpen is the global imprint of INTECHOPEN LIMITED, registered in England and Wales, registration number: 11086078, 7th floor, 10 Lower Thames Street, London,

EC3R 6AF, United Kingdom

Printed in Croatia

British Library Cataloguing-in-Publication Data

A catalogue record for this book is available from the British Library

Additional hard and PDF copies can be obtained from orders@intechopen.com

Vascular Malformations of the Central Nervous System

Edited by Bora Gürer and Pinar Kuru Bektaşoğlu

p. cm.

Print ISBN 978-1-78985-711-5

Online ISBN 978-1-78985-712-2

eBook (PDF) ISBN 978-1-83880-146-5 


\section{We are IntechOpen, \\ the world's leading publisher of Open Access books}

\section{Built by scientists, for scientists}

\section{$4,600+$}

Open access books available

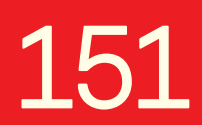

Countries delivered to

$119,000+$

International authors and editors

Our authors are among the

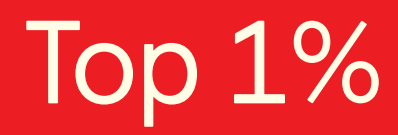

most cited scientists
$135 \mathrm{M}+$

Downloads

\section{$12.2 \%$}

Contributors from top 500 universities

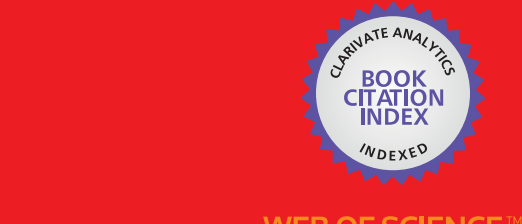

Selection of our books indexed in the Book Citation Index in Web of Science ${ }^{\mathrm{TM}}$ Core Collection (BKCI)

\section{Interested in publishing with us? \\ Contact book.department@intechopen.com}

Numbers displayed above are based on latest data collected.

For more information visit www.intechopen.com 



\section{Meet the editors}

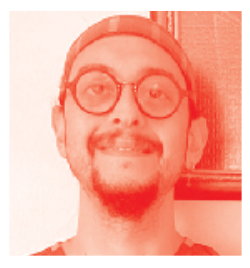

Bora Gürer is currently affiliated to Department of Neurosurgery, University of Health Sciences, Fatih Sultan Mehmet Education and Research Hospital, Istanbul, Turkey. He graduated from Dokuz Eylul University, Faculty of Medicine. Besides being the youngest associate professor of neurosurgery in Turkey, he has a keen interest in complex neurovascular, neurooncological, and skull base surgeries. He has authored numerous papers in peer-reviewed national and international journals.

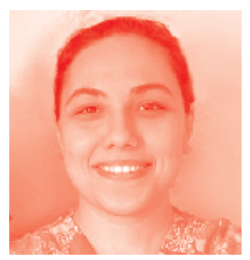

Pınar Kuru Bektaşoğlu graduated from Marmara University School of Medicine. She is currently pursuing her residency in the Department of Neurosurgery, University of Health Sciences, Fatih Sultan Mehmet Education and Research Hospital and doing her Ph.D. in the Department of Physiology, Marmara University School of Medicine. She has a keen interest in neurovascular and neuro-oncological surgery. She has authored numerous papers in peer-reviewed national and international journals. 



\section{Contents}

Preface

Section 1

General Approach for Cerebral Vascular Malformations

Chapter 1

Cerebral Arteriovenous Malformations (cAVMs): What Is New?

by Adel E. Ahmed Ganaw, Abdulgafoor M. Tharayil, Moad Ehfeda,

Mohamed Hassan Alfared, Qazi Zeeshan, Sohel M.G. Ahmed, Ali Ayyad,

Ibrahim Naemi, Hossam Algallie, Nissar Shaikh and Nabil Shallik

Chapter 2

Intensive Care Management in Cerebral Aneurysm and Arteriovenous

Malformations

by Sedef Tavukçu Özkan

Section 2

Cerebral Arteriovenous Malformations

Chapter 3

Cerebral Arteriovenous Malformation from Classification

to the Management

by Nissar Shaikh, Aisha Al-Kubaisi, Muhammad Mohsin Khan,

Adnan Khan, Zia Mahmood, Arshad Chanda, Adel Ganaw,

Gamal Al-Ameri, Mostafa Rezk, Moad Ehfeda, Muhammad Zubair,

Jazib Hassan and AR Raju Vegesna

Chapter 4

Endovascular Treatment of Cerebral Arteriovenous Malformations

by Cagin Senturk

Chapter 5

Advocating Intraluminal Radiation Therapy in Cerebral Arteriovenous

Malformation Treatment

by Nitzan Hirsh, Amir Arthur and Saar Golan 
Section 3

Blister Aneurysms

Chapter 6

Blister Aneurysms

by Themistoklis I. Papasilekas, Konstantinos M. Themistoklis,

Stefanos I. Korfias and Damianos E. Sakas

\section{Section 4}

Arteriovenous Fistulas

Chapter 7

Arteriovenous Fistulas: The Pathological Bridge

by Sara A. AbuSini, Nour A. Tashtush, Abdelwahab J. Aleshawi

and Amer A. Jaradat

Section 5

Moyamoya Disease

Chapter 8

Moyamoya Disease: A Rare Vascular Disease of the CNS

by Abdulgafoor M. Tharayil, Adel E. Ahmed Ganaw, Nissar Shaikh, Sujith M. Prabhakaran, Arshad H. Chanda, Simi Praveen, Ajith Kumar Choran and Qazi Zeeshan ul Haq

\section{Section 6}

Spinal Epidural Haematomas

Chapter 9

Spontaneous Spinal Epidural Haematomas

by David Kieser, Scheherezade Soltani, Michael Wyatt, Khoon Lim

and Sandra Kieser 


\section{Preface}

Vascular malformations of the central nervous system are challenging pathologies. Our knowledge about their biology and natural history is increasing. Diagnostic modalities help clinicians to better evaluate the individual cases, and to decide the best treatment options. Treatment alternatives are also evolving and clinicians are doing their best for each individual patient. The purpose of this book is to review the current knowledge about vascular malformations of the central nervous system, to evaluate the treatment alternatives, and to make suggestions for special considerations. We hope the informations gathered in this book will be helpful for our readers.

Pınar Kuru Bektaşoğlu, MD

Istanbul Fatih Sultan Mehmet Education and Research Hospital,

Department of Neurosurgery, Istanbul, Turkey

Marmara University School of Medicine, Değartment of Physiology,

Istanbul, Turkey

Bora Gürer, MD

Istanbul Fatih Sultan Mehmet Education and Research Hospital,

Department of Neurosurgery,

Istanbul, Turkey 

Section 1

\section{General Approach for Cerebral Vascular Malformations}





\title{
Cerebral Arteriovenous Malformations (cAVMs): What Is New?
}

\author{
Adel E. Ahmed Ganaw, Abdulgafoor M. Tharayil, \\ Moad Ehfeda, Mohamed Hassan Alfared, Qazi Zeeshan, \\ Sohel M.G. Ahmed, Ali Ayyad, Ibrahim Naemi, \\ Hossam Algallie, Nissar Shaikh and Nabil Shallik
}

\begin{abstract}
Cerebral arteriovenous malformations (cAVMs) are rare congenital anomalies of cerebral blood vessels that result from maldevelopment of the capillary bed, permitting direct communication between cerebral arteries and veins. It usually occurs in the supratentorial area of the brain; however, it can occur anywhere in the brain and spinal cord. Most of the patients with cAVMs present with a variety of complaints such as seizures, intracerebral hemorrhage, headache, and progressive focal neurological deficit. Imaging such as CT, MRI, and angiography plays a vital role in diagnosis, grading, risk assessment, and posttherapeutic follow-up. The multidisciplinary team use three therapeutic modalities in the treatment of cAVMs. This chapter reviews the clinical presentations, diagnosis, classification, and treatment of cAVMs.
\end{abstract}

Keywords: cerebral angiography, endovascular embolisation, focal neurological deficit, headache, intracerebral hemorrhage, microsurgery, radiosurgery, seizers

\section{Introduction}

Arteriovenous malformations (AVMs) are rare congenital anomalies of cerebral blood vessels with critical clinical implications. These anomalies may occur anywhere in the central nervous system (brain and spinal cord). It is a neurosurgical emergency that is characterized by abnormal vascular web, consisting of a tangle of dysplastic vessels (nidus) fed by arteries and drained by veins without intervening capillaries, generating a high flow, low resistance shunt between cerebral arteries and veins (Figure 1). They are a significant form of vascular malformation, with dilated arteries congregating from different directions and distended veins twisting from turbulence of shunted blood flow. This gives the pathology its radiological characteristic (early venous drainage) on formal catheter-based cerebral angiography. Although cAVMs are considered as a congenital cerebrovascular disease, patients may present with intracerebral hemorrhage, convulsions, chronic headache, and 


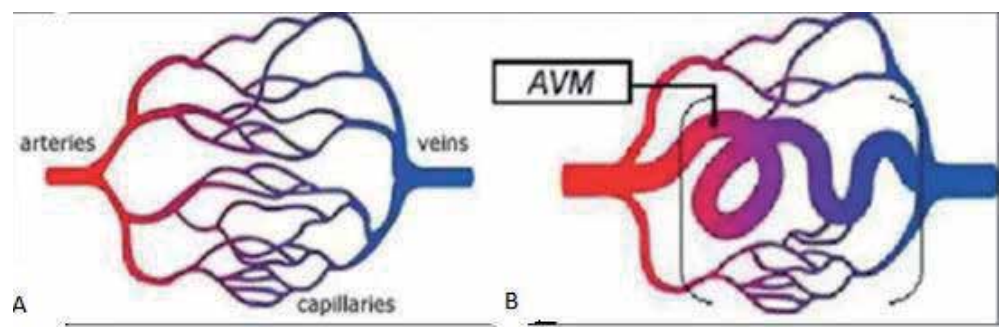

Figure 1.

(A) Normal vasculature, arteries, and veins communicate at capillary bed. (B) cAVM direct communication between high-flow arterial vessels and low-resistance venous capacitance vessels. And an arteriovenous malformation. Steal phenomenon (blood flow through low resistance shunt (low resistance) and stealing blood flow from adjacent brain) [1].

progressive neurological deficit. Some of them remain asymptomatic and are noticed only incidentally on cerebral imaging for other indications. AVMs are associated with a lifelong risk of intracerebral hemorrhage (ICH); therefore, these lesions necessitate appropriate assessment, workup, and considerations for treatment based upon many factors such as age, size, and location of the lesions [2-5].

\section{Epidemiology}

The prevalence of cAVMs is very low, and a collection of population-based studies from the literature estimates the incidence of cerebral AVMs at 0.69-1.32 per 100,000. However, they are considered as an important cause of intracerebral hemorrhage in a young age group. Bleeding rate is almost $1 \%$ per year in previously unruptured arteriovenous malformations. cAVMs cause approximately $2-4 \%$ of all hemorrhagic strokes $[2,4,6,7]$.

They are a congenital disease that is developed from indistinguishable causes in developing a fetus. While numerous mechanisms of cAVM formation have been suggested, the heterogeneity and lack of inheritance of these pathologies make their exact cause to remain idiopathic. They are associated with a few syndromes, such as hereditary hemorrhagic telangiectasia, Wyburn-Mason syndrome, von HippelLindau disease, and Sturge-Weber syndrome [2].

Cerebral AVMs generally occur as a single lesion, and multiple lesions are observed only in $9 \%$ of the patients. cAVMs are equally distributed between sexes $[8,9]$.

Patients may present at any age, although they most commonly present in the second through fourth decades of life $[6,7]$.

\section{Clinical manifestation}

cAVM patients may present with a variety of clinical symptoms such as hemorrhage, seizure, headaches, or focal neurologic deficits. The clinical presentations of cAVMs depend on the patient's age, size, location, and vascular features of the cAVMs. Some patients are asymptomatic at presentation and cAVMs are accidently discovered when imagines are performed for potentially unrelated reasons.

\subsection{Hemorrhage}

Hemorrhage is the most common initial presentation of cAVMs. Studies reported that hemorrhage is the initial presentation in $50 \%$ of patients with cAVMs. The risk of hemorrhagic stroke in cAVM patients is about $2-5 \%$ per year. It is fatal 
in $5-25 \%$ of all cAVM patients. Children more frequently present with intracerebral hemorrhage than do adults. Hypertension, previous intracerebral hemorrhage (ICH), AVMs with feeding artery aneurysm, AVMs with multiple arterial feeders, deep-seated cAVMs, and cAVMs with limited venous drainage are associated with increased risk of hemorrhagic stroke. Hemorrhage begins in the lesion itself and then accumulates, increases in size, and produces a mass effect. Therefore, hemispherical lesions generate less severe syndromes in comparison with primary hemorrhage that originates from functioning parts of the brain. Hemorrhages in polar regions such as frontal, temporal, and occipital regions may cause only minor headache, while those in deeper structures affect vital regions packed into tiny spaces and produce severe neurological manifestations. Hence, patients with ICH may present with a wide span of presentations such as sudden severe headache, drop in the level of consciousness, progressive neurological deficits, hemiparesis, aphasia, cranial nerve palsy, visual field deficits, and coma [2,9-11].

The type of the hemorrhage and clinical presentations depend on the location of cAVMs. Superficial lesions cause subarachnoid hemorrhage with low risk of cerebral vasospasm and the bleeding confined in local sulci, whereas lesions with deep drainage may vent blood in the ventricular system, causing hemohydrocephalus. Lesions embedded in the brain parenchyma cause parenchymatous hemorrhage. Figure $2[11,13]$.

\subsection{Seizure}

Seizures are the second most common presentation of cAVMs; it occurs in 20-29\% of patients with cAVMs at initial diagnosis. One study showed that the 5-year risk of first seizure was $8 \%$ (95\% CI 0-20). The risk of the seizures rose to $23 \%$ in the presence of ICH and focal neurological deficit. The seizures are frequently observed in male patients with cortical lesions, especially in the frontal and temporal lobe. Large lesions (nidus greater than $3 \mathrm{~cm}$ in diameter) and superficial venous drainage are associated with increase in seizure frequency. Posterior fossa, coexisting aneurysms, and deep locations were associated with the absence of seizures. AVM may cause focal or generalized seizures or both. The exact pathophysiology of the seizures is not completely understood. However, increased venous back pressure secondary to venous outflow obstruction may be involved. cAVM-related seizures may occur secondary to ICH, from the hemosiderin deposition, or secondary to venous hypertension, and ischemia following steal. Seizure control rate ranges between 49 and $85 \%$ according to approach (surgery, radiosurgery, or embolisation), and the highest seizure freedom has been achieved in patients with approved complete angiographic obliteration after radiosurgery $[2,9,14]$.

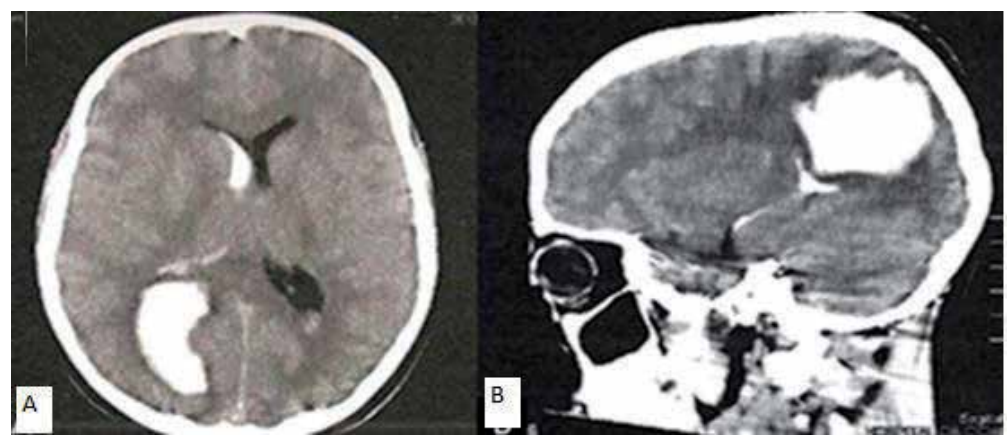

Figure 2.

(A) Axial and (B) sagittal plain CT brain of 13-year-old patient presented with sever headache; the scan demonstrates right parieto-occipital intra-axial hemorrhage with intra-ventricular extension. Urgent craniotomy was done due to deterioration of the neurological status confirming underlying AVM [12]. 


\subsection{Headache}

Approximately $0.2 \%$ of cAVM patients present with headache and normal neurologic examination. The headache associated with cAVMs is incidental, without any specifications. Headache is the presenting symptom in nearly $15 \%$ of cAVM patients without evidence of rupture. Headache can be characterized as similar to migraines with lateralization to one side $[11,13]$. The attack occurrence, aurae, duration, and pulsatile quality are all shared. Treatment of headache in AVM patients has not been studied so far. No specific medication has been exclusively useful in headache management. Expert opinions do not recommend using any vasoconstrictors in these patients as it may lead to rupture and $\mathrm{ICH}$. However, there are limited data to support this recommendation [9].

\subsection{Neurological deficit}

Neurological deficit is a relatively rare presentation of cAVMs. Patients may present with transient, progressive, permanent, or progressive focal neurological deficits not secondary to ICH or seizures. Vascular steal syndrome is assumed to be the main reason of neurologic deficit. Moreover, recurrent ICH, local mass effect, or hydrocephalus may cause neurological deficits $[2,9]$.

\section{Diagnosis of cAVMs}

Diagnosis of cAVMs must include a detailed assessment of the nidus and the adjacent structures. Plain CT is the initial diagnostic tool for cAVM patients who present with one of previously mentioned clinical manifestations, while CT angiography or digital subtraction angiography are used to locate the exact site of rupture in patients with ICH. Magnetic resonance imaging (MRI) is the recommended investigation for patients with nonhemorrhagic manifestations. Digital subtraction angiography is the investigation of choice for the characterization of the feeding arteries, nidus angioarchitecture, and draining veins and is mandatory for precise AVM grading and management planning $[2,4]$.

\subsection{Computerized tomography (CT) and related techniques}

CT scan is the first radiological investigation that is performed in patients who present with convulsions, focal neurological deficit, or clinical signs of ICH. Plain head CT is useful for confirming ICH or assessing abnormal suspicious dilated dural veins, abnormal faint hyperdense cerebral mass, and focal cerebral calcification, which are alert signs for the radiologist for the possibility of underlying cAVMs; however, negative CT scan cannot be excluding the presence of cAVMs. Compression of the nidus by hematoma impedes CT diagnosis of cAVM in patients with acute ICH. Therefore, CT angiography should be done promptly to diagnose the underlying cAVMs. CT angiogram with intravenous iodinated contrast media with images acquired by bolus tracking technique is very helpful for maximum cerebral as well as cAVM arterial opacification. The CT head angiogram is used for confirming the diagnosis, assessing the cAVM size, site, number, and origin of feeding arteries and draining vein as well as the presence of associated aneurysm and site of bleeding. The high spatial resolution of CTA allows the generation of multiplanar reformations, maximum intensity projections, and volumetric reconstructions for analysis (Figures 3-5) $[4,16,18]$. 
Cerebral Arteriovenous Malformations (cAVMs): What Is New?

DOI: http://dx.doi.org/10.5772/intechopen.90096
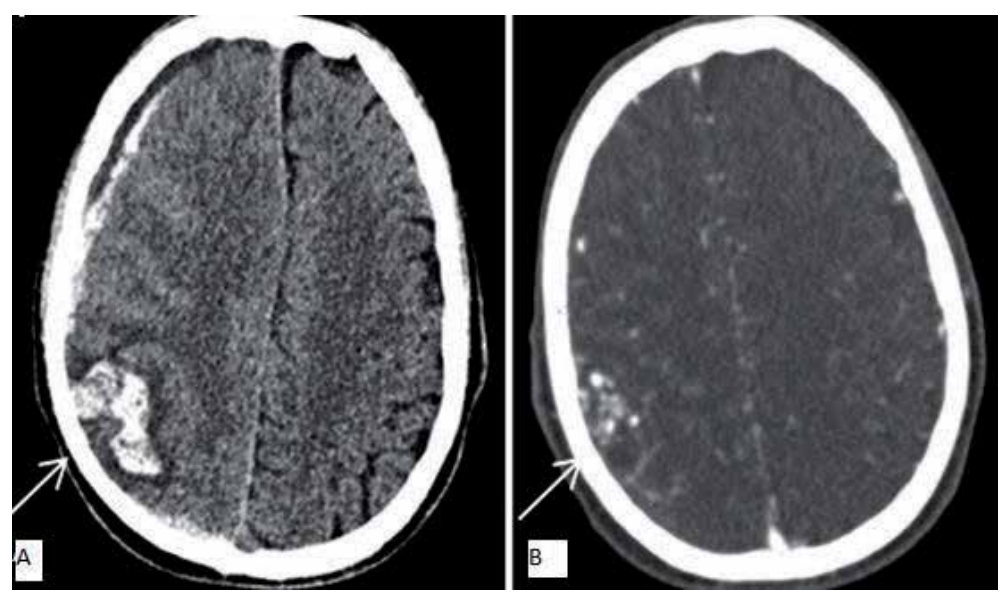

Figure 3.

Plain CT head (A) and CT head angiogram (B) of young adult patient demonstrate right parietal parenchyma brain hemorrhage (the arrow on $A$ image) and multiple serpiginous tortuous vascular malformation AVM (the arrow on the B image) [15].

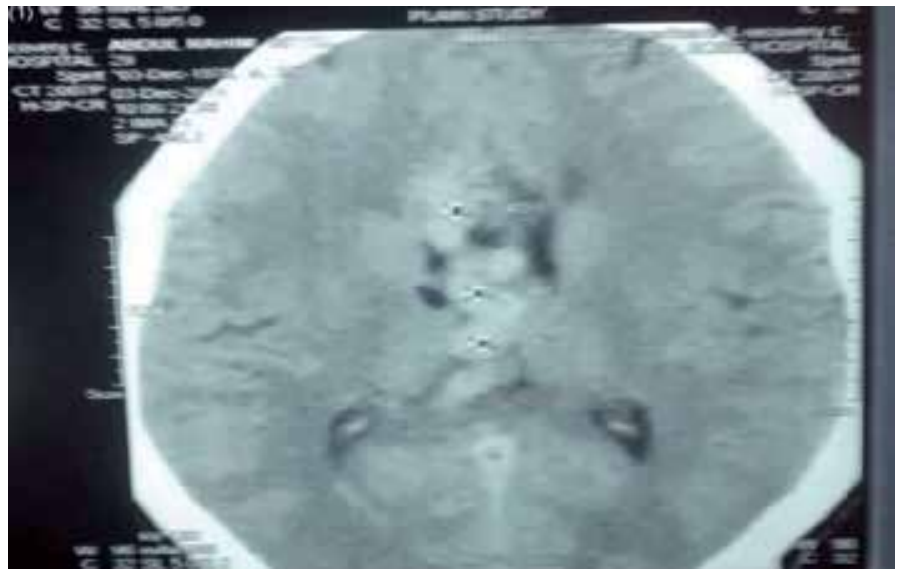

Figure 4.

Plain CT shows hyperdense tortuous structure at lateral ventricular region representing AVM [16].
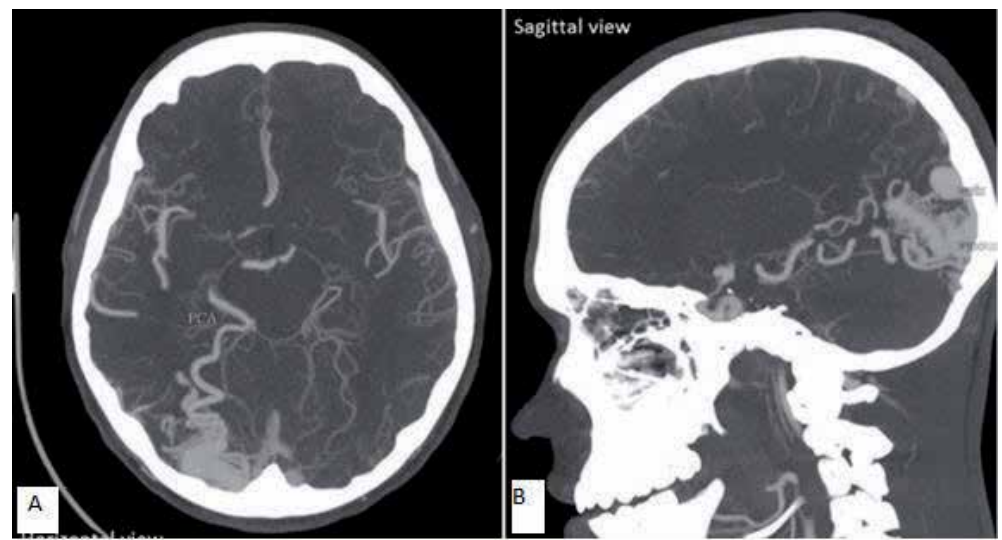

Figure 5.

CT head angiogram MIP (maximum intensity projection) axial and sagittal view ( $A$ and $B$ respectively) demonstrates right occipital lobe AVM supplied mainly by right posterior cerebral artery and drained to straight and right transverse dural sinuses [17]. 
In recent decades, the radiological equipment has showed visible development making us more aware of the pathophysiology of many diseases by using perfusion CT and MRI scan for cAVMs to provide information regarding the blood flow abnormality within and around such vascular anomalies by assessing cerebral blood flow (CBF), cerebral blood volume (CBV), and the mean transient time (MTT). Through these, we can demonstrate three different forms of extra-nidal parenchymal perfusion abnormalities:

1. Functional steal is characterized by redistribution of the blood flow from surrounding brain tissue through the arteriovenous malformation that leads to disturbance of normal cerebral perfusion. Patients with this phenomenon usually present with convulsions. CT scan shows decrease in CBV, CBF, and MTT as a result of sump effect from the artery supplying the arteriovenous malformation Figures 6 and 7.

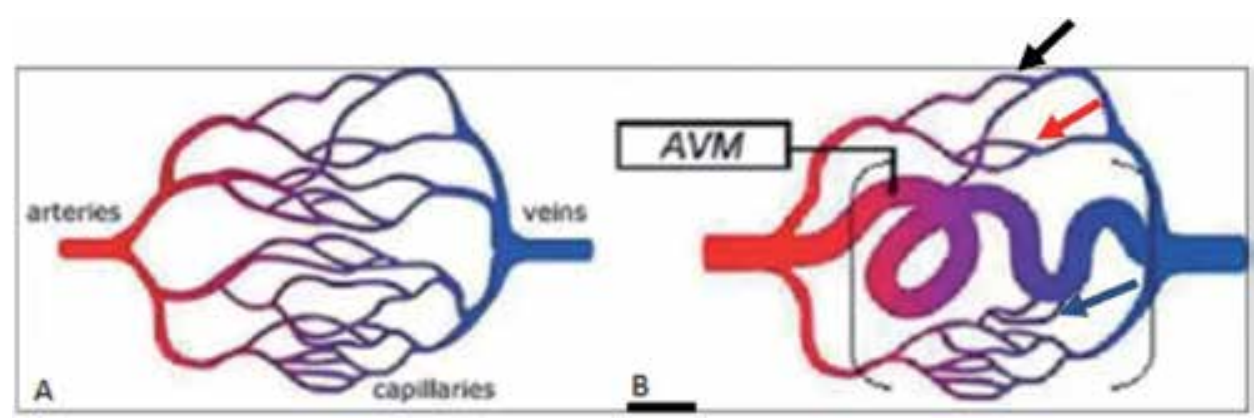

Figure 6.

Normal and Perfusion abnormalities diagram in cAVMs. (A) Illustrate normal brain tissue blood flow. (B) Illustrate the abnormal brain tissue blood flow within and adjacent to the AVM. The Black arrow represent functional steal region in which the blood shunted away to the nearby AVM. The red arrow representing ischemic steal region due to indirect collateral of the near the AVMs. The blue arrow is a brain region drained by high pressure veins of AVM leading to venous congestion [15].

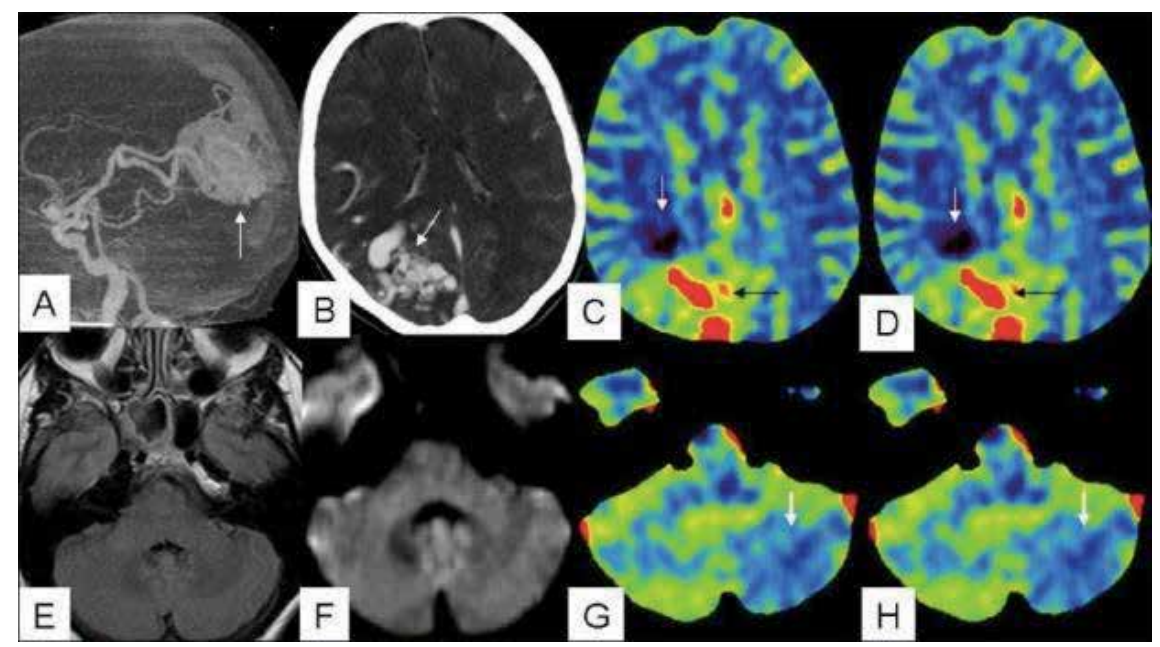

Figure 7.

Illustrate the effect of steal phenomenon in Brain's AVM on adjacent brain tissue $(A)$ and $(B)$ CT angiograms demonstrate right side occipital AVM (arrows). Selected images from brain CT perfusion (C and $D)$ shows high CBF (black arrows in $C$ ) and CBV (black arrows in D) in the nidus and low CBF (white arrows in C) and $C B V$ (white arrows in $D$ ) in the perinidal area, suggestive of perinidal ischemia. There is no evidence of cerebellar ischemia on the FLAIR (E) and diffusion $(F)$ images. CBF (arrows in $G$ ) and CBV (arrows in $H$ ) are low in the contralateral cerebellar hemisphere, suggestive of cerebellar diaschisis [19]. 
2. Ischemic steal is characterized by a reduction in CBF and CBV with increased MTT in the brain parenchyma secondary to indirect collateral flow to the shunt from nearby arteries. These patients usually present with a focal neurological deficit.

3. Venous congestion is characterized by increased CBV and MTT in remote parts of the brain as a result of the high-pressure flow in the draining veins of the AVM. These patients may present with progressive neurological deficits $[4,15,16,18]$.

\subsection{Magnetic resonance imaging (MRI) of the brain and related techniques}

MRI of the brain is very sensitive for the exact determination of the cAVM nidus location and an associated draining vein; it also has exclusive sensitivity in determining remote bleeding due to these lesions. On noncontrast brain MRI, the cAVM nidus is seen as signal void on T2-weighted images with dilated feeding arteries and draining veins, and susceptibility-weighted imaging can detect the presence of hemosiderin component of chronic bleeding as low signal intensity. The ischemic and brain steal area can appear as hyperintense T2 weighted and FLAIR. MRA also could be used to add crucial information regarding the feeding arteries, draining veins, and the presence of associated aneurysm, which appear Could be gained from CT perfusion (CTP). The same pathophysiological information that could be gained from CTP can also be done by MRI perfusion using noncontrast ALS (arterial spine labeling) technique or with the usage of contrast media Figures 8 and 9 [2, 4].

MRI of the brain is especially valuable in follow-up patients after treatment. After radiosurgery, MRI is essential to follow up regression of the nidal volume. While the MR angiography is very useful to characterize the venous drainage and other vascular features, the adjacent tissue that is exposed to the radiation field can be precisely observed for posttherapy edema or radiation necrosis $[4,16]$.

\subsection{Digital subtraction angiography (DSA)}

Digital subtraction angiography is the gold standard for assessment of nature and number of the feeding arteries, presence of flow-related aneurysms, quality of venous drainage, associated varices, and stenosis.
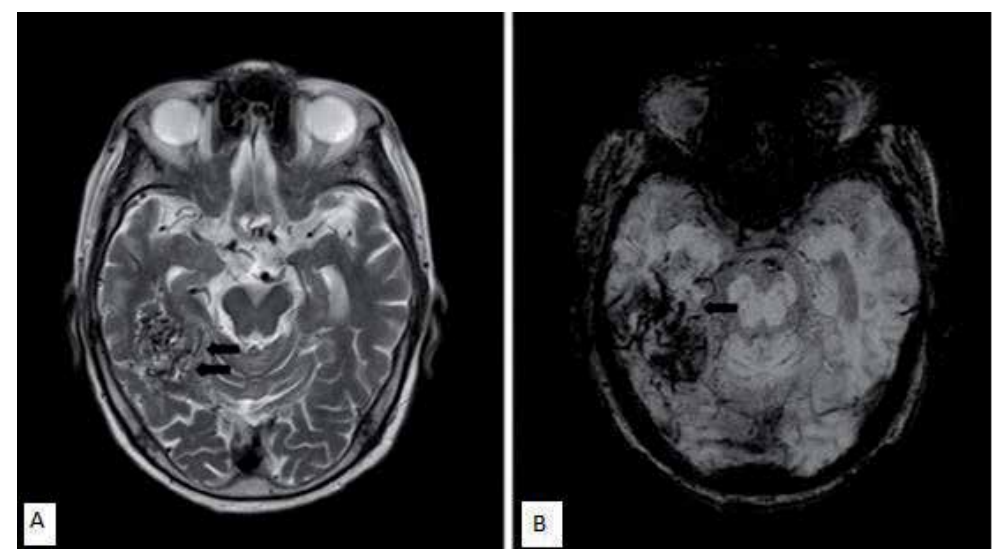

Figure 8.

MRI brain of an adult patient T2-weighted image A and SWI (susceptibility weighted image). B demonstrates right temporal T2WI and DWI serpiginous hypointensity representing AVM [20]. 

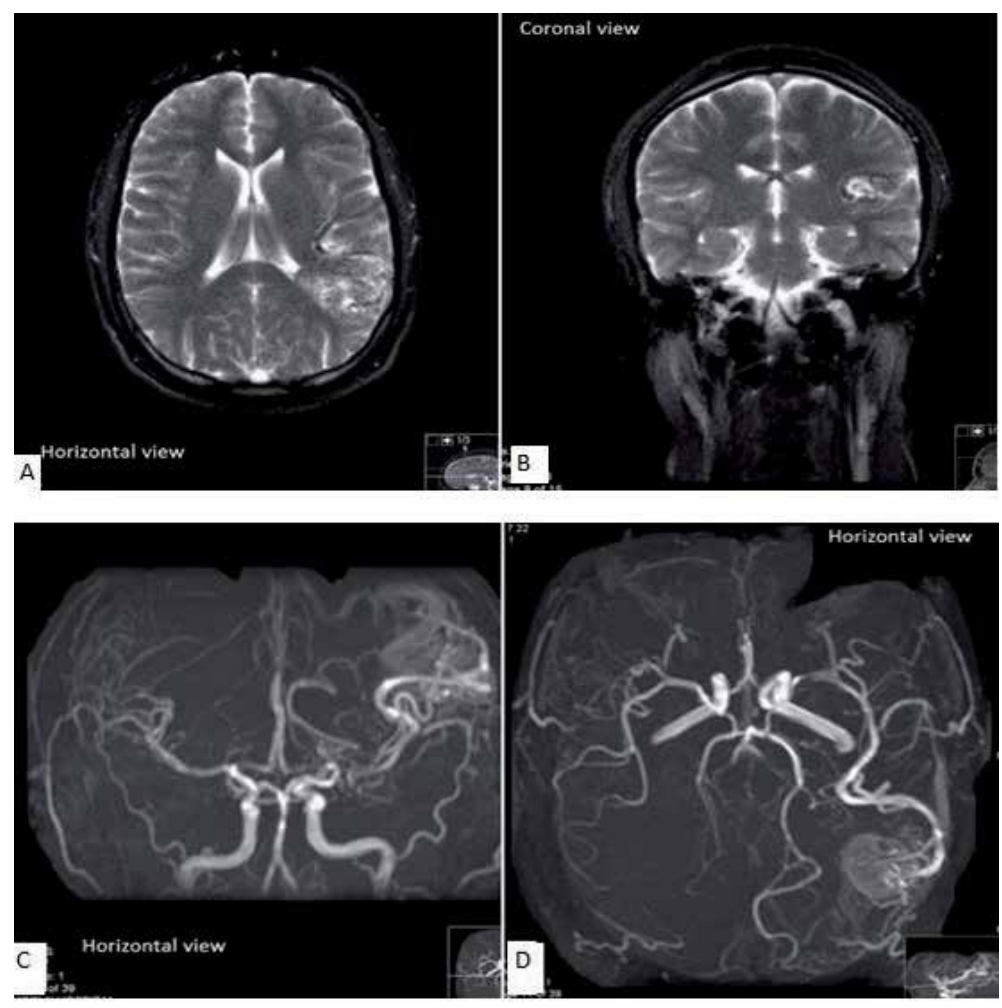

Figure 9.

MRI T2-weighted image A\&B and MRA brain C\&D images the brain demonstrate $T 2$ weighted signal void serpiginous structure at left inferior parietal lobe; on $M R A$, images show the nidus fed by arterial feeder from left middle cerebral artery and drained to superficial cortical veins to left transverse sinus [16].

Two types of feeding arteries that can be identified by DSA are as follows:

1. Direct arterial feeders: they end in the AVM; it is either large cortical arteries or small perforator coursing through white matter or ventricles.

2. Indirect arterial feeders: they supply the AVM through small branches that arise from the involved but also pass to healthy brain tissue distal to AVM.

Flow-related aneurysms in the feeding arteries usually result from amplified shear stress and considered as an indicator of vascular friability and hemorrhage risk. These aneurysms may resolve following curative treatment of the AVM. Intranidal aneurysms are usually small, less than $3 \mathrm{~mm}$, and pseudoaneurysms and can be successfully treated with embolisation.

The AVM nidus may have both fistulous and glomerular compartments; the fistulous compartments form high-flow shunt that can be treated with endovascular therapy, while the glomerular compartments form an intervening network of pathological vessels and are very difficult to treat with embolisation.

Assessment of the venous part of AVM is critical and should include the anatomy of drainage (superficial, deep, or mixed) and characteristics of the venous outflow (focal stenosis, venous pouches, and sinus stenosis or occlusion). Drainage into the deep venous system is associated with a high risk of bleeding and indicates deep location that may make surgical intervention difficult $[2,4]$. 


\section{Grading of cAVMs}

Before starting the management of cAVMs, all management risks should be considered, starting from those associated with diagnosis, surgical intervention, radiotherapy, to interventional radiology. Treating physicians should also consider both early and late complications such as hemorrhage and convulsions. There are many grading systems that may help physicians to assess the risk of these complications, take appropriate intervention for each patient, predict prognosis, and standardize patient's evaluation for scientific study purposes [21-23].

\subsection{Spetzler-Martin (S-M) grading}

In 1986, Spetzler and Martin introduced the most commonly used grading scale for cAVMs; it uses three anatomical features of AVM (nidus size, nidus location relative to the eloquent brain (Figure 10), and pattern of venous drainage) to generate five cAVM grades (Table 1).

To determine S-M grade of cAVMs, size of the nidus, venous drainage, and eloquence of adjacent brain tissue are determined in angiography, brain CT scan, and/ or MRI of the brain. A numerical value is assigned for each category, as described in Table 1. The grade is derived from adding the points awarded for each category; for example, for grade I, the lesion would be small (less than $3 \mathrm{~cm} / 1$ point), in noneloquent region ( 0 point), and have only superficial drainage ( 0 point) (Figure 11); therefore, excision of such lesion would be complete, minimally invasive, and associated with low surgical complications. Whereas for grade $\mathrm{V}$, the lesion would be large (more than $6 \mathrm{~cm} / 3$ points), sited within or immediately close to eloquent brain

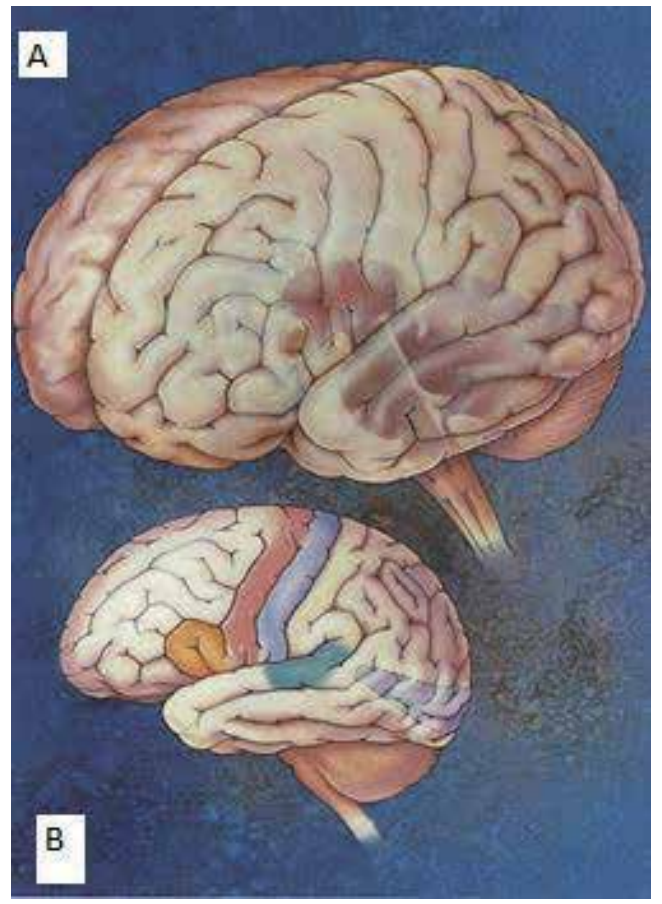

Figure 10.

The anatomic areas considered neurologically eloquent. (A) Deep eloquent area (hypothalamus, thalamus, brain stem, and cerebellar peduncle). (B) Eloquent area in cerebral cortex (sensory-motor, primary visual, and language area) [19]. 


\begin{tabular}{lc}
\hline Size of cAVM & Point \\
\hline $0-3 \mathrm{~cm}$ & 1 \\
\hline $3.1-6 \mathrm{~cm}$ & 2 \\
\hline$>6 \mathrm{~cm}$ & 3 \\
\hline Location of cAVM (eloquence area) & 0 \\
\hline No & 1 \\
\hline Yes & \\
\hline Venous drainage & 0 \\
\hline Superficial & 1 \\
\hline Deep & \\
\hline
\end{tabular}

Table 1.

Spetzler-Martin (S-M) grading scale is used to make decisions about treatment risks.

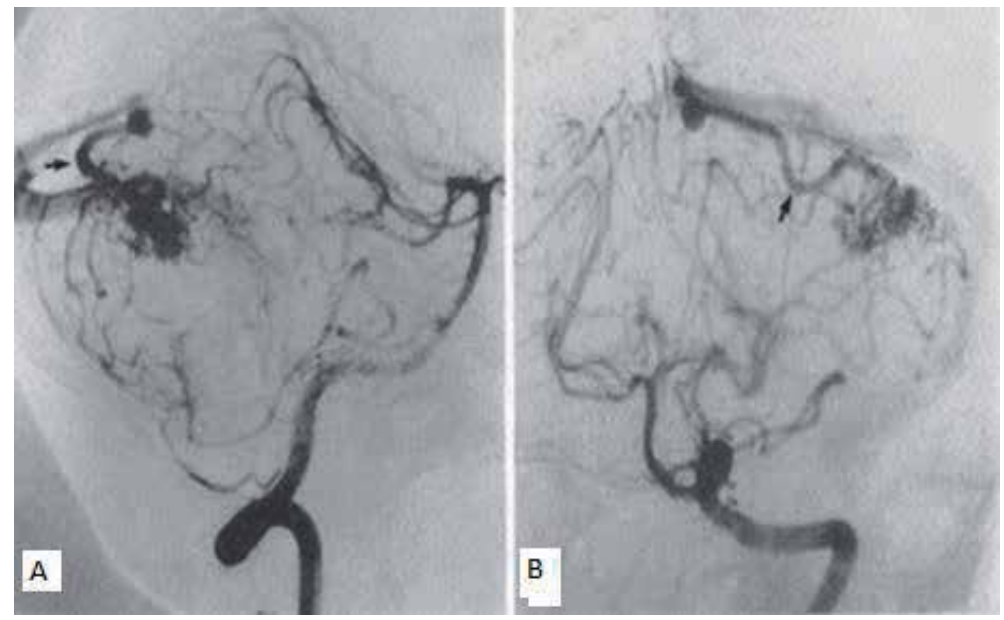

Figure 11.

Grade I AVM; (A) Vertebral angiograms. (B) Anteroposterior view cerebellar AVM less than $3 \mathrm{~cm}$ (1 point), superficial on cerebellar cortex (o point), superficial drainage (through superficial cerebellar hemisphere veins-arrows: o point) [19].

(1 point), and venous drainage would drain into deep venous system (Figure 12), so excision of such lesion is extremely difficult and associated with significant risk of bleeding and surgical morbidity and mortality. Moreover, obliteration of large cAVMs leads to sudden increase in perfusion, which leads to vasogenic edema or hemorrhage (normal perfusion pressure break through phenomenon). There are certain lesions that should not be considered for surgery such as large AVM that invades eloquent critical area or diffuse nidus that involves crucial structure such as hypothalamus or brain stem (Figure 13), so the surgical resection of such lesions would necessarily be associated with significant neurological deficit or death. These AVMs are graded under separate category (grade VI) and considered as inoperable AVM [22, 23].

The S-M grading scale was introduced to predict the outcome of surgical resection but can also be used to predict the radiosurgical outcome. Lower grades (grades I and II) are associated with a good result and less postoperative neurological deficit in comparison with grades IV and V [21-23].

Multiple retrospective studies showed that rates of poor outcome were highly correlated with S-M grade. Precisely, rates of poor outcome were calculated as follows: S-M grade I = 4\% (95\% CI, 2-7), S-M grade II = 10\% (95\% CI, 7-13), S-M 
grade III = 18\% (95\% CI, 15-22), S-M grade IV = 31\% (95\% CI, 25-37), and S-M grade $\mathrm{V}=37 \%$ (95\% CI, 26-49). These results prove that microsurgical resection is suitable for low-grade cAVMs (S-M grades I and II), whereas surgical resection of high-grade cAVMs (S-M grades IV and V) is associated with a high risk of poor patient outcome. Lawton and Davies et al. subcategorized grade III into three groups based on a specific combination of the same anatomical features (size, location, and venous drainage) to improve prediction of treatment outcome:

Group 1: Patients with S-M grade III cAVMs (a combination of small size, eloquent location, and deep venous drainage) have surgical outcomes similar to those of S-M grades I and II.
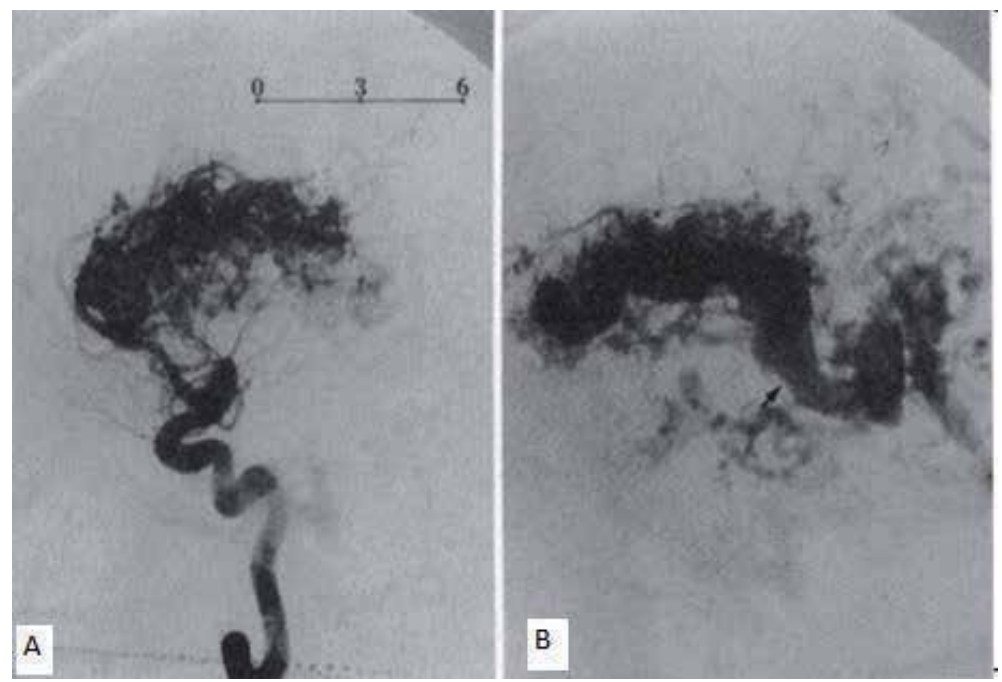

Figure 12.

Grade VAVM; (A) Carotid angiogram, arterial phase. (B) Venous phase, AVM greater than $6 \mathrm{~cm}$ (3 points), situated in corpus callosum with a deep thalamic component (eloquent: 1 point), draining into a hugely dilated internal cerebral vein (deep drainage-arrow 1 point) [19].

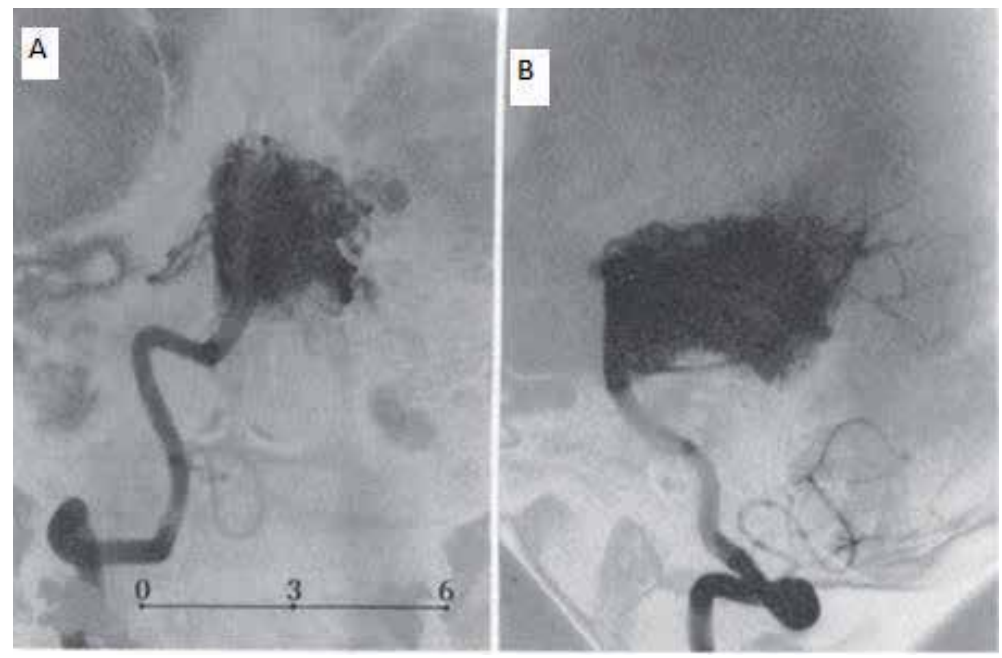

Figure 13.

Grade IV AVM (inoperable AVM), (A) Vertebral angiogram, anteroposterior view. (B) Lateral view, arterial phase this lesion fed by multiple vessels from rostral basilar artery, surrounding and invading the mesencephalon [19]. 


\begin{tabular}{lcc}
\hline Size of cAVM & Point & Patient's age \\
\hline $0-3 \mathrm{~cm}$ & 1 & $<20$ \\
\hline $3.1-6 \mathrm{~cm}$ & 2 & $20-40$ \\
\hline$>6 \mathrm{~cm}$ & 3 & $>40$ \\
\hline Location of cAVM (eloquence area) & \multicolumn{2}{c}{ Compactness } \\
\hline No & 0 & Yes \\
\hline Yes & 1 & No \\
\hline Venous drainage & \multicolumn{2}{c}{ Bleeding } \\
\hline Superficial & 0 & Yes \\
\hline Deep & 1 & No \\
\hline
\end{tabular}

Table 2.

Supplementary Spetzler-Martin or Lawton-Young cAVM grading scale.

Group 2: Patients with S-M grade III cAVMs (a combination of medium size, noneloquent location, and deep venous drainage) have a poor surgical outcome similar to that of S-M grades IV and V.

Group 3: Patients with S-M grade III cAVMs (a combination of medium size, eloquent location, and superficial venous drainage) have a poor surgical outcome similar to that of S-M grades IV and V [23].

Supplementary grading scale to traditional S-M grading scale was proposed in 2010 to enhance its predictive power for predicted outcomes after surgical resection of cAVMs. This supplemental scoring system added three factors that affect patient's outcome (age, hemorrhagic presentation, and nidus configuration) (Table 2). The supplementary grading scale named Lawton-Young supplementary grading scale has been authorized in a separate cohort of 1009 patients [21, 23].

\section{Treatment of cAVMs}

Complete resection or obliteration of the nidus and AV shunt is considered as the treatment of choice of cAVMs. Incomplete nidal resection does not decrease hemorrhage risk. However, There are few reports of partial resection may improve neurological symptoms in patients who have neurological symptoms related to hemodynamic. There are three complementary therapeutic techniques that have been developed to achieve these goals (microsurgery \pm embolization, stereotactic radiosurgery, and embolisation). The treatment plans of cAVMs should be carefully taken by a multidisciplinary team of knowledgeable and skilled physicians. They should take the size, location, and vascular features of the AVM in consideration. Moreover, risks of significant complications such as short- and long-term hemorrhage risk, feasibility, associated aneurysm, patient's age, risks of intervention, availability of interventional radiologist, and size and compactness of the nidus should be assessed. The short- and long-term hemorrhage risk is associated with a history of raptured cAVM, patient age, AVM location, size, and vascular morphological features [23-25].

Children and young adults have a long life expectancy, and the risk of intracerebral hemorrhage (ICH) is gradually increasing over the years. Therefore, curative therapy and complete obliteration are recommended whenever possible, while conservative treatment may be considered for elderly patients. The cumulative risk of hemorrhage can be calculated by the formula: lifetime risk of hemorrhage $=1-$ $(1-\mathrm{P}) \times \mathrm{N}(\mathrm{N}$ is the expected years of life remaining, and $\mathrm{P}$ is the annual probability of hemorrhage). Another more straightforward formula is as follows: lifetime risk of hemorrhage $=105$-patient's age in years. 


\subsection{Microsurgery}

Craniotomy and surgical resection of cAVMs were first introduced in the 1920s, where the outcomes were inferior. The development of angiography played an essential role in understanding vascular anatomy and hemodynamics of AVM lesions. The introduction of microsurgery and bipolar diathermy has resulted in significant improvement of morbidity and mortality in comparison to the early known surgical resections. Microsurgical excision of cAVMs is considered as the most efficient approach as it offers the best chance of immediate cure and eliminates morbidity and mortality associated with its potential rupture. Before taking patients to the operating theater for surgical resection, preoperative angiogram and MRI should be done for intraoperative stereotactic localization of the lesion. Moreover, endovascular embolisation has frequently been considered to improve surgical safety and to possibly magnify the pool of cAVMs amenable to safe surgical resection. The main goals of endovascular embolisation are elimination of arterial feeders (particularly those that would be challenging to access in early stages of surgical intervention), decrease of nidus volume that would warrant safer surgical resection, and management of high-risk angiographic characters such as feeding artery and intranidal aneurysms.

Essential steps in the resection process of AVM are wide dural opening, identification of AVM lesion, elimination of superficial feeding arteries, circumferential resection of the nidus with control of deep arterial pedicle, and transection of draining veins. When all arterial feeders to the nidus have been determined and coagulated, the direct venous drainage can be blocked and the nidus resected. Intraoperative vascular imaging, such as DSA, is beneficial to confirm complete nidus resection at the time of surgery. Furthermore, indocyanine green videoangiography with either fluorescein or indocyanine can be very useful to intraoperatively map the angioarchitecture of the lesions, including distinguishing arterial feeders from arterialized draining veins.

The surgeons should vigilantly inspect the resection bed microscopically to make sure that hemostasis has been achieved, during which the blood pressure is maintained at $15-20 \mathrm{~mm} \mathrm{Hg}$ above the baseline. Oxidized cellulose (Surgicel) can be used to line the nidus bed, and the patient's goal blood pressure should be maintained within the normal range for the first 24 hours after surgical resection.

Although the microscopic complete nidus resection has long-term durability and leads to abrupt elimination of hemorrhage risks, it is an invasive intervention and associated with neurological risks $[23,25]$.

\subsection{Stereotactic radiosurgery (SRS)}

Radiosurgery is typically reserved for the obliteration of compact cAVMs (less than $3 \mathrm{~cm}$ ) in patients who are poor surgical candidates either radiographically (Spetzler-Martin grades $>$ III) or due to existing medical comorbidities. SRS promotes endothelia cell proliferation, gradual, concentric vessel wall thickening, and ultimately luminal closure. Numerous studies about efficiency and safety of radiosurgery indicated that radiosurgery seems to be suitable for small-tomoderate volume lesions (less than $12 \mathrm{~cm}^{3}$ in volume or less than $3 \mathrm{~cm}$ in maximum diameter). Furthermore, radiosurgery is best suited for injuries situated in deep or eloquent regions of the brain. The primary goal of SRS is the obliteration of cAVMs. With obliteration, the risk of hemorrhage from cAVM nidus is eliminated. Complete obliteration of cAVM nidus after SRS is associated with fading of cAVMassociated epilepsy as well as improvement of nidus-associated neurological signs and symptoms. 


\begin{tabular}{lccccccc}
\hline Study & Number & Year & Design & Follow-up & $\begin{array}{c}\text { Obliteration } \\
\text { rate\% }\end{array}$ & $\begin{array}{c}\text { Annual } \\
\text { hemorrhage } \\
\text { rate before } \\
\text { obliteration \% }\end{array}$ & $\begin{array}{c}\text { Permanent } \\
\text { radiation } \\
\text { injury \% }\end{array}$ \\
\hline Ding et al & 444 & 2013 & Retrospective & $\begin{array}{c}86 \text { Month } \\
\text { (mean) }\end{array}$ & 62 & 1.6 & 2 \\
\hline Starke et al & 2236 & 2016 & $\begin{array}{c}\text { Multicenter } \\
\text { registry }\end{array}$ & $\begin{array}{c}7 \text { year } \\
\text { (median) }\end{array}$ & 64.7 & 1.1 & 2.7 \\
\hline Pollock & 174 & 2013 & Retrospective & $\begin{array}{c}64 \text { month } \\
\text { (mean) }\end{array}$ & 78.9 & Not reported & 4 \\
\hline
\end{tabular}

Table 3.

Radio-surgical outcomes for unruptured cAVMs [23].

Most series with long-term follow-up show obliteration in 70-80\% of cAVMs and it is achieved within 2-3 years after starting stereotactic radiosurgery (Table 3 ) $[17,20]$. The time between starting radiosurgery and complete obliteration is named the latency period; it varies from 1 to 3 years. During this latency period, the risk of hemorrhage ranges between 1 and 3\% per year. The outcome of radiosurgery has been affected by many factors such as age, nidus volume, preceding embolisation, and previous hemorrhage. Therefore, all these factors should be considered before deciding to proceed with radiosurgery.

Though preceding endovascular embolisation may shrink large nidus to a suitable target volume for SRS as well as eradicate high-risk characters associated with cAVM such as perinidal or intranidal aneurysms, it may lead to difficulties in precisely targeting residual nidus and reducing successful obliteration rates after SRS.

Radiosurgery is not entirely safe; during latency period, symptomatic changes secondary to radiation occur in 10 percent of the patients. However, permanent neurological changes are reported only in $2-3 \%$ of patients.

The main risk factors for symptomatic changes secondary to radiations are the location of AVM, target volume, and dose to surrounding healthy tissue (margin dose). The symptomatic changes secondary to radiation effect can be reduced with corticosteroids and bevacizumab.

Delayed cyst formation and radiation-induced neoplasia are uncommon although may occur after 10 years from radiosurgery [23, 26, 27].

\subsection{Embolisation}

Endovascular embolisation plays a crucial role in multidisciplinary approach to treat cAVMs. Embolisation may be considered in the following scenarios:

\subsubsection{Preoperative embolization}

The primary goal of preoperative embolisation is to facilitate surgical resection of cAVMs and reduce complication rates of microsurgery. It is instrumental in the reduction of intraoperative bleeding and postoperative complications such as healthy perfusion pressure breakthrough. The healthy brain tissue surrounding cAVMs exposes to chronic low perfusion pressure. When the lesion is wholly or partially resected, these areas are suddenly subject to normal perfusion pressure, and their autoregulation ability may be impaired. Therefore, a sudden increase in perfusion pressure may lead to brain edema, delayed hemorrhage, and seizures. Large cAVMs should go through staged embolisation to gradually 
reduce flow to the AVM before resection. Moreover, preoperative embolisation may be useful in the elimination of feeding artery pedicles that may be deep and not accessible $[23,28,29]$.

\subsubsection{Curative embolisation}

The role of endovascular embolisation as a stand-alone treatment in AVM has been reported in small case series. The introduction of detachable-tip microcatheters plays a vital role in improvement of curative embolisation rates as it facilitates extended Onyx infusion. Small lesions with few arterial feeders are most suitable for complete curative embolisation. However, most of these lesions are graded as S-M grades I and II, for which microsurgery is considered as the best therapeutic approach. Therefore, the relative risk of curative embolisation must be weighed cautiously against this traditional therapeutic approach. The durability of embolic materials and the length of follow-up are needed to establish a complete cure. AVM recurrence after successful complete angiographic elimination has been reported in various case reports [23, 30, 31].

\subsubsection{Before radiosurgery}

Embolisation before radiosurgery is commonly used when cAVM nidus is greater than $3 \mathrm{~cm}$ in diameter to reduce the size of the nidus. However, embolisation may lower rates of successful obliteration, most likely due to the artifact effect of the radiopaque embolic interfere with targeting the lesion $[23,32]$.

\subsubsection{Targeted embolization}

Targeted embolisation is used to treat high-risk angiographic features such as nidal and perinidal aneurysms and arteriovenous fistulas that may lead to rupture of AVM [33].

\subsubsection{Palliative embolization}

Infrequently, cAVMs may cause focal neurological deficits as a result of vascular steal or local venous hypertension. In these situations, decreased venous hypertension and steal by embolisation of select, high-flow feeders may improve focal neurological deficit and quality of life. However, there is no substantial evidence to support this approach and further studies are needed [23, 34, 35].

\subsubsection{Complications of embolization}

Stroke and ICH are the most common complications of endovascular embolisation. The ischemic stroke is most likely secondary to thromboembolic complications of catheterization and nontarget embolization, while ICH may occur secondary to vascular injury or AVM rupture. Arterial feeders' injury and perforation occur when a microcatheter or wire is introduced through tiny tortuous pial arteries. Accidental closure of draining veins before elimination of the nidus during embolization may cause nidal rupture and ICH $[23,35,36]$.

\section{Conclusion}

Cerebral arteriovenous malformations (cAVMs) are rare congenital disease. However, they are considered as a significant cause of hemorrhagic stroke in 
children and young adults. They account for 33\% of intracerebral hemorrhage in young patients. Although it is congenital disease, patients with cAVMs mostly present in their second and fourth decades of life. Intracerebral hemorrhage is the most common presentation of AVM patients. Imaging such as CT, MRI, and angiography is advantageous in diagnosis, grading, risk assessment, and posttherapeutic follow-up. cAVMs are graded by many grading systems. However, Spetzler-Martin grading and supplementary Spetzler-Martin grading scales are the most important grading scales as they can be used to assess risk of postoperative complications and therapeutic outcomes. Therefore, they are beneficial in deciding best therapeutic approach for each patient. There are three therapeutic modalities for management of cAVMs. The multidisciplinary team approach is the best for management of cAVM patients. The surgical excision of cAVM gives definitive therapy in cAVM patients. Radiosurgery is a curative approach in selected cAVM patients, and embolisation is rarely used as a sole therapy. It is usually used as adjunctive therapy to microsurgery and SRS.

\section{Author details}

Adel E. Ahmed Ganaw ${ }^{1 *}$, Abdulgafoor M. Tharayil ${ }^{1}$, Moad Ehfeda ${ }^{1}$, Mohamed Hassan Alfared ${ }^{2}$, Qazi Zeeshan ${ }^{1}$, Sohel M.G. Ahmed ${ }^{1}$, Ali Ayyad ${ }^{3}$, Ibrahim Naemi ${ }^{1}$, Hossam Algallie ${ }^{1}$, Nissar Shaikh ${ }^{1}$ and Nabil Shallik ${ }^{1}$

1 Anesthesiology, Intensive Care and Perioperative Medicine, Hamad General Hospital, Hamad Medical Corporation, Qatar

2 Radiology Department, Hamad General Hospital, Hamad Medical Corporation, Qatar

3 Neurosurgery Department, Hamad General Hospital, Hamad Medical Corporation, Qatar

*Address all correspondence to: aganaw@hamad.qa

IntechOpen

(C) 2019 The Author(s). Licensee IntechOpen. This chapter is distributed under the terms of the Creative Commons Attribution License (http://creativecommons.org/licenses/ by/3.0), which permits unrestricted use, distribution, and reproduction in any medium, provided the original work is properly cited. (cc) BY 


\section{References}

[1] Pandhare MN, Jyoti DB, Suresh RB. Acquired arteriovenous malformation of lip occurring as occupational hazard: A case report with review of literature. Journal of Oral and Maxillofacial Pathology. 2018;22(2):287

[2] Osbun JW. Arteriovenous and cavernous malformations. In: Handbook of Clinical Neurology. Vol. 143. 3rd series. B.V USA: Elsevier; 2017. DOI: 10.1016/B978-0-444-63640-9.00003-5

[3] Ondra SL, Troupp H, George ED. The natural history of symptomatic arteriovenous malformations of the brain: A 24-year follow-up assessment. Journal of Neurosurgery. 1990;73(3):387-391

[4] Lawton MT. Brain arteriovenous malformations. Nature Reviews Disease Primers. 2015;28:15008. DOI: 10.1038/ nrdp. 2015.8

[5] Berman MF, Sciacca RR, PileSpell-man J. The epidemiology of brain arteriovenous malformations. Neurosurgery. 2000;47:389-397

[6] Hofmeister C. Demographic, morphological, and clinical characteristics of 1289 patients with brain arteriovenous malformation. Stroke. 2000;31:1307-1310

[7] Kim H. Untreated brain arteriovenous malformation: Patientlevel meta-analysis of hemorrhage predictors. Neurology. 2014;83:590-597

[8] Willinsky RA, Lasjaunias P, Terbrugge K, Burrows P. Multiple cerebral arteriovenous malformations (AVMs). Review of our experience from 203 patients with cerebral vascular lesions. Neuroradiology. 1990;32:207

[9] Mohr JP, Kejda-Scharler J, PileSpellman J. Diagnosis and treatment of arteriovenous malformations. Current
Neurology and Neuroscience Reports. 2013;13(2):324

[10] Hetts SW, Cooke DL, Nelson J. Influence of patient age on angioarchitecture of brain arteriovenous malformations. AJNR. American Journal of Neuroradiology. 2014;35(7):1376-1380

[11] Stapf C, Mast H, Sciacca RR. Predictors of hem-orrhage in patients with untreated brain arteriovenous mal-formation. Neurology.

2006;66(9):1350-1355

[12] Manzato LB, Vanzin JR, Frighetto L, Filho PM, Pedrolo E, et al. Multiple cerebral arteriovenous malformation: Report of two cases and treatment strategy. Journal of Neurology and Neurophysiology. 2017;3(1):1-4. DOI: $10.16966 / 2379-7150.138$

[13] Choi JH, Mast H, Sciacca RR. Clinical outcome after first and recurrent hemorrhage in patients with untreated brain arteriovenous malformation. Stroke. 2006;37:1243-1247

[14] Ding D, Starke RM, Quigg M. Cerebral arteriovenous malformations and epilepsy, part 1: Predictors of seizure presentation. World Neurosurgery. 2015;84(3):645-652

[15] July J, Wahjoepramono EJ. editors. Gamma knife surgery for AVM.

Neurovascular Surgery. 2019:275-283.

DOI: $10.1007 / 978-981-10-8950-3-32$

[16] Rajesh RU, George T, Manickam A. Cerebral arterio venous malformation. International Journal of Clinical \& Medical Images. 2015;2(9):1-2. DOI: 10.4172/2376-0249.1000369

[17] Zahouani T, Lopez S, StandishParkin L, et al. High grade incidental arteriovenous malformation. Annals 
of Clinical and Laboratory Research. 2017;5:1

[18] Kim DJ, Krings T. Whole-brain perfusion CT patterns of brain arteriovenous malformations: A pilot study in 18 patients. AJNR. American Journal of Neuroradiology.

2011;32:2061-2066

[19] Shiva Shankar JJ, Lum C. Whole brain perfusion on a 320-slice CT scanner. Indian Journal of Radiology and Imaging. 2011;21:209-214

[20] Roh K, Kang H, Kim I. Clinical application of neuroimaging with susceptibility weighted imaging: Review article. Journal of the Korean Society of Magnetic Resonance in Medicine. 2014;18(4):290-302

[21] Poncea FA, Spetzler RF. Arteriovenous malformations: Classification to cure. Clinical Neurosurgery. 2011;58:10-12

[22] Spetzler RF, Martin NA. Proposed grading system for arteriovenous malformation. Journal of Neurosurgery. 1986;65:476-483

[23] Derdeyn CP et al. Management of brain arteriovenous malformations. A scientific statement for healthcare professionals from the American Heart Association/American Stroke Association. Stroke. 2017:200-224. DOI: 10.1161/STR. 0000000000000134

[24] Solomon RA, Connolly ES. Arteriovenous malformations of the brain. Review article. The New England Journal of Medicine. 2017;376:19

[25] Barr JC, Ogilvy CS. Selection of treatment modalities or observation of arteriovenous malformations. Neurosurgery Clinics of North America. 2012;23:63

[26] Pan HC, Sheehan J. Late cyst formation following gamma knife surgery of arteriovenous malformation. Journal of Neurosurgery. 2005;103(suppl):124-127

[27] Starke RM, Yen CP, Chen CJ, Ding D, Mohila CA, Jensen ME, et al. An updated assessment of the risk of radiation-induced neoplasia after radiosurgery of arteriovenous malformations. World Neurosurgery. 2014;82:395-401. DOI: 10.1016/j. wneu.2013.210.02.008

[28] Gutiérrez-González R, Gil A, Serna C, López-Ibor L, Boto GR. Normal per-fusion pressure breakthrough phenomenon: What still remains unknown. British Journal of Neurosurgery. 2012;26:403-405. DOI: $10.3109 / 02688697.2011 .633637$

[29] Spetzler RF, Wilson CB, Weinstein P, Mehdorn M, Townsend J, Telles D. Normal perfusion pressure breakthrough theory. Clinical Neurosurgery. 1978;25:651-672

[30] Moon K, Levitt MR, Almefty RO, Nakaji P, Albuquerque FC,

Zabramski JM, et al. Safety and efficacy of surgical resection of unruptured low-grade arteriovenous malformations from the modern decade. Neurosurgery. 2015;77:948-952. DOI: $10.1227 /$ NEU.0000000000000968

[31] Bauer AM, Bain MD, Rasmussen PA. Onyx resorbtion with AVM re-canalization after complete AVM obliteration. Interventional Neuroradiology. 2015;21:351-356. DOI: $10.1177 / 1591019915581985$

[32] Kano H, Kondziolka D, Flickinger JC, Park KJ, Iyer A, Yang HC, et al. Stereotactic radiosurgery for arteriovenous malformations after embolization: A case-control study. Journal of Neurosurgery. 2012;117:265275. DOI: 10.3171/2012.4.JNS111935

[33] Brown RD, Wiebers DO, Forbes GS. Unruptured intracranial 
aneu-rysms and arteriovenous malformations: Frequency of intracranial hem-orrhage and relationship of lesions. Journal of Neurosurgery. 1990;73:859-863. DOI: 10.3171/jns.1990.73.6.0859

[34] Batjer HH, Devous MD, Seibert GB, Purdy PD, Ajmani AK, Delarosa M, et al. Intracranial arteriovenousmalformation: Relationships between clinical and radiographic factors and ipsilateral steal severity. Neurosurgery. 1988;23:322-328

[35] Kusske JA, Kelly WA. Embolization and reduction of the "steal" syndrome in cerebral arteriovenous malformations. Journal of Neurosurgery. 1974;40:313321. DOI: $10.3171 /$ jns.1974.40.3.0313

[36] Asadi H, Kok HK, Looby S, Brennan P, O'Hare A, Thornton J. Outcomes and complications after endovascular treatment of brain arteriovenous malformations: A prognostication attempt using artificial intelligence. World Neurosurgery. 2016;96:562-569.e1. DOI: 10.1016/j. wneu.2016.09.086 



\title{
Intensive Care Management in Cerebral Aneurysm and Arteriovenous Malformations
}

\author{
Sedef Tavukçu Özkan
}

\begin{abstract}
Aneurysmal subarachnoid hemorrhage is an important group of intracranial hemorrhage with a high risk of disability and mortality. The initial amount of bleeding, rebleeding, and delayed cerebral ischemia are considered as the most important factors in determining the prognosis of aneurysm-induced bleeding. In arteriovenous malformations, its location and deep venous drainage play a role in the prognosis. Cardiac complications, neurogenic pulmonary edema, hypertension, hyperglycemia, infections, and prolonged mechanical ventilation in aneurysmal subarachnoid hemorrhage lead to morbidity and mortality. Aneurysm bleeding control, appropriate fluid replacement to ensure euvolemia, when necessary external ventricular drainage and/or decompressive craniectomy, mannitol or hypertonic saline application, and infection control are the main principles of treatment.
\end{abstract}

Keywords: aneurysmal subarachnoid hemorrhage, cerebral arteriovenous malformation, cerebral vasospasm, delayed cerebral ischemia, intracranial pressure

\section{Definition and etiology}

The modern definition of cerebral artery aneurysms began in 1874 with Duret's description of the middle cerebral artery (MCA). It is known that $20 \%$ of all aneurysms are composed of MCA aneurysms and $90 \%$ of aneurysms originate from anterior circulation. It is more common in women aged 35-60 years [1]. Subarachnoid hemorrhage (SAH) is a major health problem worldwide with a high mortality rate. Despite a 17\% decrease in case fatality in the last three decades associated with improved management strategies, 30-day mortality and sudden death rate unfortunately are still high, around 35 and $15 \%$, respectively [2].

Cerebral arteriovenous malformations (AVM) are composed of a complexity of abnormal arteries and veins and are a major source of brain hemorrhage, resulting in morbidity and mortality, representing a diagnostic and therapeutic challenge in young adults. Cerebral AVM generally represents 1 and $3 \%$ of the total annual risk for epilepsy and bleeding in patients with AVM. The rate risk of bleeding in undamaged AVMs is $2.2 \%$ per year, and the rate of torn lesions is $4.5 \%$ per year. Important risk factors for the development of bleeding due to AVM include deep localization, deep venous drainage, associated aneurysms, pregnancy, age, and gender. Disconnection of arteriovenous malformations from circulation and prevention 


\begin{tabular}{ll}
\hline Formation of intracranial aneurysms & Hypertension \\
Smoking \\
Chronic alcohol use \\
Family history \\
Female sex \\
Polycystic kidney disease \\
Marfan syndrome \\
Ehler-Danlos syndrome \\
Fibromuscular dysplasia \\
Neurofibromatosis type I \\
\hline
\end{tabular}

Table 1.

Risk factors for the formation of intracranial aneurysms.

\begin{tabular}{ll}
\hline Rupture of intracranial aneurysms & Female sex \\
Smoking \\
Hypertension \\
Cocaine abuse \\
Sympathomimetic drug use \\
Posterior circulation aneurysms \\
Type of aneurysms \\
Giant aneurysms \\
\hline
\end{tabular}

Table 2.

Risk factors for the rupture of intracranial aneurysms.

of epileptic seizures remain the mainstay of treatment. The size, location, and presence of deep venous drainage (using the Spetzler-Martin score) determine the mortality and management of the arteriovenous malformations.

The most common causes of aneurysm remain hemodynamic instability, degenerative changes, vascular injury, atherosclerosis, vasculopathy, high flow, arteriovenous malformation, and fistula. In addition, the important risk factors for the development of cerebral aneurysms are hypertension, smoking, chronic alcohol use, family history of intracranial aneurysms in first-degree relatives, and female sex. Autosomal dominant polycystic kidney disease is an inherited systemic disorder that is strongly associated with intracranial aneurysms. Autosomal dominant polycystic kidney disease has a prevalence rate 2-4 times higher than the general population. Other conditions such as Marfan syndrome, Ehler-Danlos syndrome type IV, neurofibromatosis type I, hereditary hemorrhagic telangiectasia, Moyamoya disease, pseudoxanthoma elastum, and fibromuscular dysplasia are weakly associated with intracranial aneurysms. Multiple aneurysms are detected in $10-30 \%$ of cases. Both localization and type of aneurysm are important considerations in describing the risk for rupture. Aneurysm rupture is directly proportional to size, and ruptured aneurysm causes subdural, subarachnoid, or intracranial hematoma. $25 \%$ of the cases with ruptured aneurysm are lost and $50 \%$ of them have partial or complete recovery. Overall incidence of mortality and complications in ruptured aneurysm is $80 \%$. Risk factors for the formation of and rupture of intracranial aneurysms are shown in Tables 1 and 2 [1, 3, 4].

Aneurysms are classified according to their shape or size.

According to the shape:

1. Saccular (traumatic, mycotic, oncotic, flow-dependent vasculopathy, druginduced).

2. Fusiform. 
3. Dissecting.

According to the size:

1. Small $(<15 \mathrm{~mm})$.

2. Large (15-25 mm).

3. Giant $(25-50 \mathrm{~mm})$.

4. Super giant $(>50 \mathrm{~mm})$.

Saccular berry aneurysms account for $90 \%$ of the total aneurysm morphology, and their rupture is the most common cause of SAH. Fusiform aneurysms account for the remaining $10 \%$, and their most common location is posteriorly. Atherosclerosis and dissection are found to be responsible as possible mechanisms for formation of fusiform aneurysms.

\section{Clinical presentation of subarachnoid hemorrhage}

The most common clinical reflection of aneurysm is SAH; it can occur in several ways: headache, bilateral temporal hemianopsia and bilateral lower limb weakness, unilateral nerve palsy, facial or orbital pain, nosebleed, progressive vision loss and/or ophthalmoplegia, and symptoms of brain stem dysfunction. Patients with complex middle cerebral artery aneurysms may present with intracranial hemorrhage, mass effects, epilepsy, or cerebral ischemia; in addition, the aneurysm may be incidentally discovered. A high mortality rate of $65-85 \%$ within 2 years has been reported, and aneurysm rupture survivors are often left with severe neurological deficits [5-9].

In the presence of rupture, aneurysmal SAH may cause coma in $20-30 \%$ of patients. In "poor-grade" patients, neurologic findings may include extensor posturing and loss of upper brain stem reflexes, and further progression may occur in hours from the ictus. A wide light-fixed pupil may indicate oculomotor palsy from a ruptured posterior communicating aneurysm or lateral brain stem displacement from a hematoma in the temporal or frontal lobe. Pinpoint pupils may indicate the presence of an acute hydrocephalus with ventricles often filled with blood. The causes of coma after aneurysmal SAH are shown in Table 3.

The severity of SAH is clinically assessed and graded using either the Hunt and Hess classification or the World Federation of Neurosurgeons (WFNS) scale (Tables 4 and 5). The WFNS, widely used, is a combination of focal neurological deficits and the Glasgow coma scale (GCS). Higher grades on both scales are

- Diffuse ischemic global cortical injury

- Early cerebral edema

- Acute hydrocephalus

- Hematoma in the temporal lobe and brain stem shift

- Hematoma in the pons

- Nonconvulsive status epilepticus

Table 3.

Causes of coma in aneurysmal subarachnoid hemorrhage. 


\begin{tabular}{ll}
\hline Grade & Clinical description \\
\hline 0 & Unruptured aneurysm \\
\hline 1 & Asymptomatic or minimal headache and slight nuchal rigidity \\
\hline 2 & Moderate/severe headache, nuchal rigidity, cranial nerve palsy \\
\hline 3 & Drowsiness, confusion, or mild focal deficit \\
\hline 4 & Stupor, severe hemiparesis, vegetative disturbance \\
\hline 5 & Deep coma, decerebrate rigidity, moribund appearance \\
\hline $\begin{array}{l}\text { Hypertension, diabetes, arteriosclerosis, chronic pulmonary disease, or vasospasm assigns patient to the next less } \\
\text { favorable category. }\end{array}$
\end{tabular}

Table 4.

Modified Hunt and Hess classification [13].

\begin{tabular}{lll}
\hline Grade & Glasgow coma scale score & Motor deficit \\
\hline I & 15 & Absent \\
\hline II & $14-13$ & Absent \\
\hline III & $14-13$ & Present \\
\hline IV & $12-7$ & Present/Absent \\
\hline V & $6-3$ & Present/Absent \\
\hline Source: Ref. [10]. & \\
\hline
\end{tabular}

Table 5.

World Federation of neurosurgeons scale.

\begin{tabular}{cllll}
\hline Grade & Fisher scale & $\begin{array}{l}\text { \% with } \\
\text { symptomatic } \\
\text { vasospasm }\end{array}$ & $\begin{array}{l}\text { Modified Fisher } \\
\text { scale }\end{array}$ & $\begin{array}{l}\text { \% with } \\
\text { symptomatic } \\
\text { vasospasm }\end{array}$ \\
\hline I & Focal thin & $21 \%$ & $\begin{array}{l}\text { Focal or diffuse } \\
\text { thin SAH, no IVH }\end{array}$ & $24 \%$ \\
\hline II & Diffuse thin SAH & $25 \%$ & $\begin{array}{l}\text { Focal or diffuse } \\
\text { thin SAH, with } \\
\text { IVH }\end{array}$ & $33 \%$ \\
\hline III & Thick SAH present & $37 \%$ & $\begin{array}{l}\text { Thick SAH present, } \\
\text { no IVH }\end{array}$ & $33 \%$ \\
\hline IV & $\begin{array}{l}\text { Focal or diffuse thin SAH } \\
\text { with significant ICH or }\end{array}$ & $31 \%$ & $\begin{array}{l}\text { Thick SAH present, } \\
\text { with IVH }\end{array}$ & $40 \%$ \\
& IVH & & & \\
\hline Source: Ref. [11]. & & &
\end{tabular}

Table 6.

Fisher and modified fisher grading scale.

associated with the worst outcomes. The WFNS classification provides a more objective assessment. The first computerized tomography scan uses the Fisher grading scale to determine the amount, localization, prognosis, and severity of bleeding (Table 6). The risk of vasospasm is high in patients with Grade III and IV SAH.

The overall mortality in patients with SAH is over $30 \%$, and approximately $10-20 \%$ of survivors show functional dependence despite intensive neurological care. Several extensive studies have been conducted to improve intensive neurological care in patients with SAH [12]. Poor-grade aneurysmal SAH (WFNS Grades IV and V) 
reflect $20-30 \%$ of all aneurysmal SAH. Mortality is commonly associated with neurological injury resulting from the initial bleeding and rebleeding and from delayed cerebral ischemia (DCI). The volume of initial hemorrhage and initial neurological status following SAH remain major factors for mortality. Elderly patients and patients with coexisting medical conditions are at higher risk. The clinical goal is to prevent rebleeding and DCI [13-15].

In general, the prognosis of aneurysmal SAH is considered to be inversely related to grading at first presentation. Aggressive early interventions such as emergency surgical clip application or endovascular treatment of the aneurysm can lead to positive outcomes in poor-grade aneurysmal SAH patients. Subarachnoid hemorrhage patients can be followed with conservative treatment without invasive intervention. External ventricular drainage is a frequently preferred method in the case of hydrocephalus which can be observed during this clinical follow-up. In a multicenter, prospective observational study conducted with 324 patients, the relationship between the potential clinical risk factors and the prognosis of aneurysmal SAH in intracranial aneurysm patients was investigated. Results showed that age, female gender, ventilated respiratory status, pupil dilatation, low GCS, WFNS grade, intraventricular hemorrhage, higher Fisher grade, higher Modified Fisher grade, and a relatively poor outcome in aneurysmal SAH patients receiving conservative treatment play a major role. There are many studies showing that age has a strong relationship with clinical outcomes in aneurysmal SAH patients [16-23]. Global cerebral edema occurs in up to $57 \%$ of patients suffering from subarachnoid hemorrhage and is associated with prolonged hospital stay and poor outcome. The pathogenesis of brain injury after intracerebral hemorrhage is thought to be due to mechanical damage followed by ischemic, cytotoxic, and inflammatory changes in the underlying and surrounding tissue. Typically, a sudden rise in intracranial pressure at the moment of rupture reduces cerebral perfusion globally in both hemispheres and results in marked ischemic changes. Intraventricular extension of the hemorrhage and hydrocephalus may be a cause of coma, and thus, an improvement may be seen after ventriculostomy $[3,24,25]$.

\section{Delayed cerebral ischemia}

Rebleeding after SAH remains one of the most serious early complications; the reported incidence is up to $15 \%$ in the first 24 hours, and the mortality rate is approximately 70\% [26-28]. Prognosis is closely related to initial bleeding, rebleeding, and DCI. The presence of intraventricular and intracerebral hemorrhage also adversely affects prognosis. Cardiac symptoms and neurogenic pulmonary edema are considered indicative of SAH severity. Hypernatremia after SAH is considered as a poor neurological marker. DCI generally begins approximately 3 days after bleeding, and its most severe presence is 1 week after bleeding. Smoking is considered as a risk factor for the development of DCI. The mechanism of DCI is not completely known, but the degree of initial bleeding is likely to be multifactorial with the severity of a function. It is also known that DCI occurs in cerebral regions without signs of angiographic vasospasm. The general recommendation is that angiographic vasospasm should not be treated in the absence of DCI.

DCI is usually treated by administration of nimodipine, via effect of maintenance of normal circulating blood volume and induced hypertension. Nimodipine is a calcium antagonist, and its oral administration is useful in the treatment of vasospasm and DCI. IV application of nimodipine is not recommended. High-dose IV nicardipine $(0.15 \mathrm{mg} / \mathrm{kg} / \mathrm{hr}$ for $14 \mathrm{~d})$ has been shown to reduce symptomatic vasospasm but not positively affect the 3 -month neurological outcome in a prospective double-blind randomized controlled trial [4, 29-35]. 
The clinical goal is to prevent rebleeding and DCI, because, in patients with $\mathrm{SAH}, \mathrm{DCI}$ is considered the most important preventable cause of death and poor neurological prognosis. Delayed cerebral ischemia affects up to $30 \%$ of patients and leaves the majority of survivors with motor deficits, cognitive dysfunction, and reduced quality of life. The risk of developing DCI is associated with the severity of initial bleeding [36].

In patients with GCS 8 or less with intracranial hemorrhage, endotracheal intubation should be considered to protect the airway or to clear tracheal secretions. Intubation should be performed with a rapid induction with minimal effect on hemodynamics and not increasing intracranial pressure. Drugs used in induction should be preferred accordingly (e.g., propofol). It should be remembered that propofol may cause a drop in blood pressure. Preventive isotonic liquid bolus should be applied if necessary. Post-expiratory positive pressure (PEEP) up to $12 \mathrm{mmHg}$ is considered safe as long as the mean arterial pressure is maintained [37].

\section{Factors determining prognosis in subarachnoid hemorrhage}

The prognosis after SAH may vary from severe disability to death, depending on the complications usually seen in the first 2 weeks associated with the severity of bleeding.

Common causes of this deterioration include neurological events such as hydrocephalus, seizures, ischemia, and systemic conditions, such as fever and infections, respiratory failure, and electrolyte abnormalities. The level of consciousness is considered the most important early predictor of outcome. For these reasons, patients presenting with a GCS score of less than 13 have traditionally been defined as having poor-grade SAH (classified as grade 4 and 5 according to the Hunt and Hess or the WFNS grading scales 5 or more recently as VASOGRADE-Red 6). Brain injury refers to the acute consequences of $\mathrm{SAH}$-associated sudden increase of intracranial pressure (ICP), which can cause decreased cerebral perfusion and transient global cerebral ischemia. The global cerebral ischemia can result in transient loss of consciousness or progressive intracranial hypertension [2, 38-41].

\section{Complications and current treatment approaches}

During aneurysmal SAH, extravasation of high-pressure arterial blood in the subarachnoid space is associated with a sudden ICP increase that, if severe and sustained, may compromise cerebral perfusion, causing global cerebral ischemia and early brain injury. Recently, the treatment of hypertension in intracranial hemorrhage patients has been discussed with INTERACT and ATACH training. The American Heart Association/American Stroke Association and Neurocritical Care guidelines include mean arterial blood pressure monitor, unsafe aneurysm types, and 110 or $160 \mathrm{~mm} \mathrm{Hg}$ (or both) of the systolic blood bridge. Remember to keep it below $180 \mathrm{~mm} \mathrm{Hg}$. After aneurysm treatment, these parameters should not be made in such a way that spontaneous high blood pressure may be beneficial [29, 42-44].

Intracranial hypertension (ICP of at least $20 \mathrm{~mm} \mathrm{Hg}$ ) is a relatively common complication of SAH, especially in patients presenting with poor neurological condition. Multiple factors such as cerebral edema, intraparenchymal hematoma, acute communicating hydrocephalus, intraventricular hemorrhage, aneurysm rerupture, complications related to aneurysm treatment, early brain injury, and DCI may contribute to the development of intracranial hypertension. High ICP is associated with severe regulation of brain metabolism, increased risk of neurological deterioration, 
and poor outcome, particularly in the absence of medical treatment. An ICP greater than $20 \mathrm{~mm} \mathrm{Hg}$ is considered as an independent predictor of severe disability and death in aneurysmal SAH. Critical cerebral perfusion pressure levels (less than $70 \mathrm{~mm} \mathrm{Hg}$ ) are significantly associated with cerebral infarction after SAH [45-51].

If the autoregulation mechanism is intact when intracranial pressure rises, the body tries to keep the cerebral blood flow (CBF) constant. As the ICP rises, the brain perfusion pressure drops. Systemic vascular resistance decreases, and vasodilatation occurs at the limits of autoregulation to keep CBF stable. Cerebral blood flow is mainly regulated by arterial carbon dioxide tension $\left(\mathrm{PaCO}_{2}\right)$. Abnormal $\mathrm{PaCO}_{2}$ levels are considered to cause major changes in $\mathrm{CBF}$ through vasoconstriction and vasodilation, respectively, possibly contributing to further brain injury [52-56].

Main management principles of intracranial hypertension after SAH are traditionally guided by the literature on traumatic brain injury, due to high numbers. It should be noted that pathophysiology is completely different in our scenario. The role of therapies such as hyperosmolar agents, hypothermia, barbiturates and decompressive craniotomy is not clear in SAH patients with intracranial hypertension resistant to first-line therapies. The first approach to elevated ICP is cerebral venous drainage, normoventilation $\left(\mathrm{PaCO}_{2}: 35-40 \mathrm{~mm} \mathrm{Hg}\right)$, and positioning bed height from $30^{\circ}$ to $45^{\circ}$. During sedation and aspiration of tracheal secretions and physiotherapy, neuromuscular blocking agents should be added if necessary. However, the role of these drugs for ICP management has not been fully established, and some authors report that they may be more harmful than useful [57].

The use of hyperosmolar agents, such as mannitol and hypertonic saline, are current popular options in the treatment of high ICP in SAH.

Studies have shown that hypertonic saline is effective in controlling ICP and improving cerebral blood flow. The last treatment steps at highly resistant ICP include barbiturate, induced hypothermia, and decompressive craniectomy. Therapeutic hypothermia has been shown to be effective in controlling ICP in SAH but has not been shown to be associated with improved functional outcome and low mortality rates.

There are studies showing that hypertonic saline is more effective than mannitol in lowering ICP in traumatic brain injury. But however, there is no specific recommendation to select hypertonic saline or mannitol as the first line for patients with high ICP caused by traumatic brain injury [58-66]. Recent literature reports effectivity of hypertonic saline like mannitol in reducing of ICP in SAH. However further studies are needed to evaluate safest and optimal dose concentration and impact on improvement of outcomes.

Decompressive craniectomy, an important approach in refractory ICP in SAH patients, is often discussed in patients with poor prognosis. Decompressive craniectomy has been associated with decreased mortality, significant decrease in ICP, increased cerebral oxygenation, and increased cerebral metabolism in many studies. However, severe disability or death was also observed in patients undergoing decompressive craniectomy for refractory ICP [67-72].

The hemodynamic approach, known as triple $\mathrm{H}$ therapy, has played a very important role in SAH treatment for many years. However, its safety and efficacy are discussed due to complications that may develop. In patients with $\mathrm{SAH}$, a bolus normal saline fluid application is known to increase cerebral blood flow in areas of cerebral ischemia. The main purpose of fluid treatment in SAH is to maintain euvolemia and normal circulating blood volume. It should be noted that uncontrolled hypervolemia and hemodilution do not improve cerebral oxygen formation and may cause adverse events [73-77].

Noradrenaline perfusion may be added to the treatment to provide normotension in cases where appropriate blood pressure is not achieved despite fluid replacement 
or in conditions limiting fluid therapy such as heart failure. If the pathology persists after blood pressure therapy in neurological examination, intravenous angioplasty or salvage therapy with intravenous infusion may be helpful. Prophylactic use of angioplasty is not recommended. Cardiac complications after SAH may vary from benign electrocardiogram changes to cardiogenic shock. Positive troponin value is common after SAH and is considered a good indicator of left ventricular dysfunction, which increases the risk of hypotension, pulmonary edema, and cerebral infarction. Treatment is symptomatic and most patients have spontaneous recovery within 2 weeks. However, aggressive ICU management may be necessary for severely impaired left ventricular functions. Dobutamine, levosimendan, milrinone, and even an intra-aortic balloon pump may be added to the treatment to maintain cerebral blood flow. It is known that the risk of heart failure and pulmonary complications is much higher in patients with low-grade SAH. Hypovolemia and pulmonary edema are known to increase the risk of delayed cerebral ischemia in this patient group. Long-term intensive care hospitalization may be required in SAH patients. This may result in pulmonary complications such as hospital-acquired pneumonia, cardiogenic or neurogenic pulmonary edema, aspiration pneumonia, and pulmonary embolism, which occurs in approximately $30 \%$ of patients.

Acute respiratory distress syndrome occurs in $27 \%$ of SAH patients and is an independent predictor of outcome. However, diuretics can be dangerous because of the risk of brain ischemia caused by hypovolemia. Early pulmonary edema and late pulmonary edema after SAH are caused by heart failure and inflammatory (i.e., non-cardiogenic) conditions, respectively.

Measurement of extravascular lung water index, cardiac index, and pulmonary vascular permeability index with Pulse Contour Cardiac Output (PiCCO) is considered to be useful in the identification of pulmonary edema in SAH patients [78-95]. Neither statin therapy nor magnesium infusions should be initiated for delayed cerebral ischemia. Cerebral vasospasm is just one component of delayed cerebral edema.

Hyponatremia is common in subarachnoid hemorrhage and is associated with longer hospitalization time, but not increased mortality. Sodium abnormalities are common and carry a risk of poor prognosis in acute $\mathrm{SAH}$ patients. We performed a 10-year observational sodium study. Hyponatremia was defined as serum sodium ( $\mathrm{sNa}$ ) concentration below $135 \mathrm{mmol} / \mathrm{L}$, whereas hypernatremia as sNa above $150 \mathrm{mmol} / \mathrm{L}$. Our 10-year targeted sodium management regimen in acute SAH patients showed that dysnatremias were frequent, predominantly hyponatremic state, due to cerebral salt wasting syndrome (CSW) and not syndrome of inappropriate antidiuretic hormone secretion (SIADH). Hypernatremia was shown to be an independent risk factor for inpatient mortality and poor outcome. The standard sodium protocol lowered the frequency of SIADH, which was encountered in only one patient over 5 years. However, it did not significantly reduce the incidence and outcome improvement of hyponatremia. Hypernatremia occurred more often and had a higher mortality and worse outcome than hyponatremia, but these patients were neurologically worse upon its onset. Hyponatremia is the most common electrolyte imbalance that occurs in $50 \%$ of patients after SAH. Two mechanisms are accepted for hyponatremia after SAH: CSW and SIADH. Cerebral salt wasting syndrome and SIADH have different pathogenesis. However, it is not always easy to distinguish in the clinic, and they can be observed in the same patient [96-100]. 


\section{Author details}

Sedef Tavukçu Özkan ${ }^{1,2}$

1 Department of Anesthesiology and Reanimation, İstinye University School of Medicine, Istanbul, Turkey

2 ICU, VM Medical Park Hospital Pendik, Istanbul, Turkey

*Address all correspondence to: sedefto@gmail.com

\section{IntechOpen}

(C) 2019 The Author(s). Licensee IntechOpen. This chapter is distributed under the terms of the Creative Commons Attribution License (http://creativecommons.org/licenses/ by/3.0), which permits unrestricted use, distribution, and reproduction in any medium, provided the original work is properly cited. (cc) BY 


\section{References}

[1] Sarıhasan B. Nöroanestezi. Serebral Anevrizmalar ve AVM da Anestezi Yönetimi. In: Sarıhasan B, Madenoğlu H, Şatırlar ZO, Türe H, Karacalar KS, editors. Kayseri: M GrupMatbaacılık; 2017. pp. 235-247

[2] de Oliveira Manoel AL, Goffi A, Marotta TR, Schweizer TA, Abrahamson S, Macdonald RL. The critical care management of poor-grade subarachnoid haemorrhage. Critical Care. 2016;20:21

[3] Wijdicks EFM. The comatose patient. In: Comatose and Aneurysmal Subarachnoid Hemorrhage. New York: Oxford University Press; 2008. pp. 268-274

[4] D’Souza S. Aneurysmal subarachnoid hemorrhage. Journal of Neurosurgical Anesthesiology. 2015;27(3):222-240

[5] Zhu W, Liu P, Tian Y, et al. Complex middle cerebral artery aneurysms: A new classification based on the angioarchitecture and surgical strategies. Acta Neurochirurgica. 2013;155:1481-1491

[6] Choi IS, David C. Giant intracranial aneurysms: Development, clinical presentation and treatment. European Journal of Radiology. 2003;46:178-194

[7] Drake CG, Peerless SJ. Giant fusiform intracranial aneurysms: Review of 120 patients treated surgically from 1965 to 1992. Journal of Neurosurgery. 1997;87:141-162

[8] Gomes F, Dujovny M, Umansky F, et al. Microsurgical anatomy of the recurrent artery of Heubner. Journal of Neurosurgery. 1984;60:130-139

[9] Gomes FB, Dujovny M, Umansky F, et al. Microanatomy of the anterior cerebral artery. Surgical Neurology. 1986;26:129-141
[10] Drake CG. Report of World Federation of Neurological Surgeons Committee on a Universal SAH grading scale. The Journal of Neurosurgery. 1988;68:985

[11] Claassen J, Bernardini GL, Kreiter K, Bates J, Du YE, Copeland D, et al. Effect of cisternal and ventricular blood on risk of delayed cerebral ischemia after subarachnoid hemorrhage: The Fisher scale revisited. Stroke. 2001;32(9):2012-2020

[12] Yokoyama S, Hifumi T, Okazaki T, et al. Association of abnormal carbon dioxide levels with poor neurological outcomes in aneurysmal subarachnoid hemorrhage: A retrospective observational study. Journal of Intensive Care. 2018;6:83

[13] Rosen DS, Macdonald RL. Subarachnoid hemorrhage grading scales: A systematic review. Neurocritical Care. 2005;2:110-118

[14] Broderick JP, Brott TG, Duldner JE, et al. Initial and recurrent bleeding are the major causes of death following subarachnoid hemorrhage. Stroke. 1994;25:1342-1347

[15] Kassell NF, Torner JC, Haley EC Jr, Adams HP, Kongable GL. The international cooperative study on the timing of aneurysm surgery. Part 1 : Overall management results. Journal of Neurosurgery. 1990;73:18-36

[16] Zheng K, Zhong M, Zhao B, et al. Poor-grade aneurysmal subarachnoid hemorrhage: Risk factors affecting clinical outcomes in intracranial aneurysm patients in a multi-center study. Frontiers in Neurology. 2019;10:123

[17] Hutchinson PJ, Seeley HM, Kirkpatrick PJ. Factors implicated in deaths from subarachnoid haemorrhage: 
Are they avoidable? British Journal of Neurosurgery. 1998;12:37-40

[18] Ransom ER, Mocco J, Komotar RJ, et al. External ventricular drainage response in poor grade aneurysmal subarachnoid hemorrhage: Effect on preoperative grading and prognosis. Neurocritical Care. 2007;6:174-180

[19] Schuss P, Konczalla J, Platz J, et al. Aneurysm-related subarachnoid hemorrhage and acute subdural hematoma: Single center series and systematic review. Journal of Neurosurgery. 2013;118:984-990

[20] Van den Berg R, Foumani M, Schroder RD, et al. Predictors of outcome in World Federation of Neurologic Surgeons grade V aneurysmal subarachnoid hemorrhage patients. Critical Care Medicine. 2011;39:2722-2727

[21] Wostrack M, Sandow N, Vajkoczy P, et al. Subarachnoid haemorrhage WFNS grade V: Is maximal treatment worthwhile? Acta Neurochirurgica. 2013;155:579-586

[22] HutterBO,Kreitschmann-AndermahrI, Gilsbach JM. Health-related quality of life after aneurysmal subarachnoid hemorrhage: Impacts of bleeding severity, computerized tomography findings, surgery, vasospasm, and neurological grade. Journal of Neurosurgery. 2001;94:241-251

[23] Hutter BO, KreitschmannAndermahr I, Mayfrank L, et al. Functional outcome after aneurysmal subarachnoid hemorrhage. Acta Neurochirurgica. Supplement. 1999;72:157-174

[24] Bernstein JE, Savla P, Dong F, et al. Inflammatory markers and severity of Intracerebral. Cureus. 2018;10:123

[25] Rass V, Ianosi BA, Wegmann A, et al. Delayed resolution of cerebral edema is associated with poor outcome after nontraumatic subarachnoid hemorrhage. Stroke. 2019;50(4):828-836

[26] Germans MR, Coert BA, Vandertop WP, et al. Time intervals from subarachnoid hemorrhage to rebleed. Journal of Neurology. 2014;261:1425-1431

[27] Fujii Y, Takeuchi S, Sasaki O, et al. Ultra-early rebleeding in spontaneous subarachnoid hemorrhage. Journal of Neurosurgery. 1996;84:35-42

[28] Starke RM, Connolly ES, Participants in the International Multidisciplinary Consensus Conference on the Critical Care Management of Subarachnoid Hemorrhage. Rebleeding after aneurysmal subarachnoid hemorrhage. Neurocritical Care. 2011;15:241-246

[29] Connolly ES Jr, Rabinstein AA, Carhuapoma JR, Derdeyn CP, Dion J, Higashida RT. Guidelines for the management of aneurysmal subarachnoid hemorrhage: A guideline for healthcare professionals from the American Heart Association/American Stroke Association. Stroke. 2012;43:1711-1737

[30] Diringer MN, Bleck TP, Claude Hemphill JIII, et al. Neurocritical Care Society. Critical care management of patients following aneurysmal subarachnoid hemorrhage: Recommendations from the neurocritical care society's multidisciplinary consensus conference. Neurocritical Care. 2011;15:211-240

[31] DeRooji NK, Rinkel GJ, Dankbaar JW, et al. Delayed cerebral ischemia after subarachnoid hemorrhage: A systemic review of clinical, laboratory, and radiological predictors. Stroke. 2013;44:43-54

[32] Dorhout Mees SM, Rinkel GJE, Feigin VL, et al. Calcium antagonists for 
aneurysmal subarachnoid hemorrhage. Stroke. 2008;39:514-515

[33] Barker FG II, Ogilvy CS. Efficacy of prophylactic nimodipine for delayed ischemic deficit after subarachnoid hemorrhage: A metaanalysis. Journal of Neurosurgery. 1996;84:405-414

[34] Haley EC Jr, Kassell NF, Torner JC. A randomized controlled trial of highdose intravenous nicardipine in aneurysmal subarachnoid hemorrhage. A report of the cooperative aneurysm study. Journal of Neurosurgery. 1993;78:537-547

[35] Güçyetmez B. Klinik Yoğun Bakım. Nörolojik Problemler. Subaraknoid Kanamaya Yaklaşım. In: Albert RK, Slutsky A, Ranieri M, Takala J, Torres A, Akpir K, Tuğrul S, editors. İstanbul: İstanbul Tip Kitabevi; 2009. pp. 405-415

[36] Francoeur CL, Mayer SA. Management of delayed cerebral ischemia after subarachnoid hemorrhage. Critical Care. 2016;20(1):277

[37] Çolak T, Küçükceran K. Critical Care Emergency Medicine. İntraserebral Hemoraji. In: Farcy DA, Chıu CW, Marshall JP, Osborn TM, Cander B, Gül M, Çakır ZG, Okumuş M, editors. İstanbul: İstanbul Tip Kitabevleri. 2. Baskı; 2018. pp. 249-359

[38] Ropper AH. Lateral displacement of the brain and level of consciousness in patients with an acute hemispheral mass. The New England Journal of Medicine. 1986;314:953-958

[39] Ropper AH, Shafran B. Brain edema after stroke. Clinical syndrome and intracranial pressure. Archives of Neurology. 1984;41:26-29

[40] Zazulia AR, Diringer MN, Derdeyn CP, Powers WJ. Progression of mass effect after intracerebral hemorrhage. Stroke. 1999;30:1167-1173
[41] Corry JJ, Varelas P, Abdelhak T, et al. Variable change in renal function by hypertonic saline. World Journal of Critical Care Medicine. 2014;3(2):61-67

[42] Diringer MN, Bleck TP, Hemphill C, et al. Neurocritical care society: Critical care management of patients

following aneurysmal subarachnoid hemorrhage: Recommendations from the neurocritical care society's multidisciplinary consensus conference. Neurocritical Care. 2011;15:211-240

[43] Steiner T, Juvela S, Unterberg A, et al. European stroke organization guidelines for the management of intracranial aneurysms and subarachnoid haemorrhage. Cerebrovascular Diseases. 2013;35:93-112

[44] Ryttlefors M, Howells T, Nilsson P, et al. Secondary insults in subarachnoid hemorrhage: Occurrence and impact on outcome and clinical deterioration. Neurosurgery. 2007;61:704-705

[45] Mack WJ, King RG, Ducruet AF, et al. Intracranial pressure following aneurysmal subarachnoid hemorrhage: Monitoring practices and outcome data. Neurosurgical Focus. 2003;14:e3

[46] Bailes JE, Spetzler RF, Hadley MN, et al. Management morbidity and mortality of poor-grade aneurysm patients. Journal of Neurosurgery. 1990;72:559-566

[47] Zoerle T, Lombardo A, Colombo A, et al. Intracranial pressure after subarachnoid hemorrhage. Critical Care Medicine. 2015;43:168-176

[48] Heuer GG, Smith MJ, Elliott JP, et al. Relationship between intracranial pressure and other clinical variables in patients with aneurysmal subarachnoid hemorrhage. Journal of Neurosurgery. 2004;101:408-416 
[49] Nagel A, Graetz D, Schink T, et al. Relevance of intracranial hypertension for cerebral metabolism in aneurysmal subarachnoid hemorrhage. Journal of Neurosurgery. 2009;111:94-101

[50] Soehle M, Chatfield DA, Czosnyka M, et al. Predictive value of initial clinical status, intracranial pressure and transcranial Doppler pulsatility after subarachnoid haemorrhage. Acta Neurochirurgica. 2007;149:575-583

[51] Väth A, Kunze E, Roosen K, Meixensberger J. Therapeutic aspects of brain tissue $\mathrm{pO}_{2}$ monitoring after subarachnoid hemorrhage. Acta Neurochirurgica. Supplement. 2002;81:307-309

[52] Yokoyama S, Hifumi T, Okazaki T, et al. Association of abnormal carbon dioxide levels with poor neurological outcomes in aneurysmal subarachnoid hemorrhage: Retrospective observational study. Journal of Intensive Care. 2018;6:83

[53] Kinoshita K. Traumatic brain injury: Pathophysiology for neurocritical care. Journal of Intensive Care. 2016;4:29

[54] Westermaier T, Stetter C, Kunze E, et al. Controlled hypercapnia enhances cerebral blood flow and brain tissue oxygenation after aneurysmal subarachnoid hemorrhage: Results of a phase 1 study. Neurocritical Care. 2016;25:205-214

[55] Pynnonen L, Falkenbach P, Kamarainen A, et al. Therapeutic hypothermia after cardiac arrest - cerebral perfusion and metabolism during upper and lower threshold normocapnia. Resuscitation. 2011;82:1174-1179

[56] Floyd TF, Clark JM, Gelfand R, et al. Independent cerebral vasoconstrictive effects of hyperoxia and accompanying arterial hypocapnia at 1 ATA. Journal of Applied Physiology. 2003;1985(95):2453-2461
[57] Sanfilippo F, Santonocito C, Veenith T, Astuto M, Maybauer MO. The role of neuromuscular blockade in patients with traumatic brain injury: A systematic review. Neurocritical Care. 2015;22:325-334

[58] Li M, Chen T, Chen SD, et al. Comparison of equimolar doses of mannitol and hypertonic saline for the treatment of elevated intracranial pressure after traumatic brain injury: A systematic review and metaanalysis. Medicine (Baltimore). 2015;94(17):e736

[59] Gu J, Huang H, Huang Y, et al. Hypertonic saline or mannitol for treating elevated intracranial pressure in traumatic brain injury: A metaanalysis of randomized controlled trials. Neurosurgical Review. 2019;42(2):499-509

[60] Tseng MY, Al-Rawi PG, Czosnyka M, et al. Enhancement of cerebral blood flow using systemic hypertonic saline therapy improves outcome in patients with poor-grade spontaneous subarachnoid hemorrhage. Journal of Neurosurgery. 2007;107:274-282

[61] Al-Rawi PG, Tseng MY, Richards HK, et al. Hypertonic saline in patients with poor-grade subarachnoid hemorrhage improves cerebral blood flow, brain tissue oxygen, and pH. Stroke. 2009;41:122-128

[62] Tseng M-Y, Al-Rawi PG, Pickard JD, et al. Effect of hypertonic saline on cerebral blood flow in poor-grade patients with subarachnoid hemorrhage. Stroke. 2003;34:1389-1396

[63] Suarez JI, Qureshi AI, Parekh PD, et al. Administration of hypertonic (3\%) sodium chloride/acetate in hyponatremic patients with symptomatic vasospasm following subarachnoid hemorrhage. Journal of Neurosurgical Anesthesiology. 1999;11:178-184 
[64] Bentsen G, Breivik H, Lundar T, et al. Hypertonic saline (7.2\%) in 6\% hydroxyethyl starch reduces intracranial pressure and improves hemodynamics in a placebo-controlled study involving stable patients with subarachnoid hemorrhage. Critical Care Medicine. 2006;34:2912-2917

[65] Mak CHK, Lu YY, Wong GK. Review and recommendations on management of refractory raised intracranial pressure in aneurysmal subarachnoid hemorrhage. Vascular Health and Risk Management. 2013;9:353-359

[66] Inamasu J, Ichikizaki K. Mild hypothermia in neurologic emergency: An update. Annals of Emergency Medicine. 2002;40:220-230

[67] Buschmann U, Yonekawa Y, Fortunati M, et al. Decompressive hemicraniectomy in patients with subarachnoid hemorrhage and intractable intracranial hypertension. Acta Neurochirurgica. 2007;149:59-65

[68] Schirmer CM, Hoit DA, Malek AM. Decompressive hemicraniectomy for the treatment of intractable intracranial hypertension after aneurysmal subarachnoid hemorrhage. Stroke. 2007;38:987-992

[69] Stuart RM, Claassen J, Schmidt M, et al. Multimodality neuromonitoring and decompressive hemicraniectomy after subarachnoid hemorrhage. Neurocritical Care. 2011;15:146-150

[70] Jaeger M, Soehle M,

Meixensberger J. Effects of decompressive craniectomy on brain tissue oxygen in patients with intracranial hypertension. Journal of Neurology, Neurosurgery, and Psychiatry. 2003;74:513-515

[71] Stiefel MF, Heuer GG, Smith MJ, et al. Cerebral oxygenation following decompressive hemicraniectomy for the treatment of refractory intracranial hypertension. Journal of Neurosurgery. 2004;101:241-247

[72] Nagel A, Graetz D, Vajkoczy P, et al. Decompressive craniectomy in aneurysmal subarachnoid hemorrhage: Relation to cerebral perfusion pressure and metabolism. Neurocritical Care. 2009;11:384-394

[73] de Oliveira Manoel AL, Turkel-Parrella D, Duggal A, et al. Managing aneurysmal subarachnoid hemorrhage: It takes a team.

Cleveland Clinic Journal of Medicine. 2015;82:177-192

[74] Macdonald RL. Delayed neurological deterioration after subarachnoid haemorrhage. Nature Reviews. Neurology. 2013;10:44-58

[75] Egge A, Waterloo K, Sjøholm H, et al. Prophylactichyperdynamicpostoperative fluid therapy after aneurysmal subarachnoid hemorrhage: A clinical, prospective, randomized, controlled study. Neurosurgery. 2001;49:593-605

[76] Lennihan L, Mayer SA, Fink ME, et al. Effect of hypervolemic therapy on cerebral blood flow after subarachnoid hemorrhage: A randomized controlled trial. Stroke. 2000;31:383-391

[77] Jost SC, Diringer MN, Zazulia AR, et al. Effect of normal saline bolus on cerebral blood flow in regions with low baseline flow in patients with vasospasm following subarachnoid hemorrhage. Journal of Neurosurgery. 2005;103:25-30

[78] Kassell NF, Peerless SJ, Durward QJ, et al. Treatment of ischemic deficits from vasospasm with intravascular volume expansion and induced arterial hypertension. Neurosurgery. 1982;11:337-343

[79] Kimball MM, Velat GJ, Hoh BL, Participants in the International Multidisciplinary 
Consensus Conference on the Critical Care Management of Subarachnoid Hemorrhage. Critical care guidelines on the endovascular management of cerebral vasospasm. Neurocritical Care. 2011;15:336-341

[80] Zwienenberg-Lee M, Hartman J, Rudisill N, et al. Effect of prophylactic transluminal balloon angioplasty on cerebral vasospasm and outcome in patients with fisher grade III subarachnoid hemorrhage: Results of a phase ii multicenter, randomized, clinical trial. Stroke. 2008;39:1759-1765

[81] Mayer SA, LiMandri G, Sherman D, et al. Electrocardiographic markers of abnormal left ventricular wall motion in acute subarachnoid hemorrhage. Journal of Neurosurgery. 1995;83:889-896

[82] Taccone FS, Lubicz B, Piagnerelli M, et al. Cardiogenic shock with stunned myocardium during triple- $\mathrm{H}$ therapy treated with intra-aortic balloon pump counterpulsation. Neurocritical Care. 2008;10:76-82

[83] Deibert E, Barzilai B, Braverman AC, et al. Clinical significance of elevated troponin I levels in patients with nontraumatic subarachnoid hemorrhage. Journal of Neurosurgery. 2003;98:741-746

[84] Banki N, Kopelnik A, Tung P, et al. Prospective analysis of prevalence, distribution, and rate of recovery of left ventricular systolic dysfunction in patients with subarachnoid hemorrhage. Journal of Neurosurgery. 2006;105:15-20

[85] Levy ML, Rabb CH, Zelman V, et al. Cardiac performance enhancement from dobutamine in patients refractory to hypervolemic therapy for cerebral vasospasm. Journal of Neurosurgery. 1993;79:494-499

[86] Busani S, Rinaldi L, Severino C, et al. Levosimendan in cardiac failure after subarachnoid hemorrhage. The Journal of Trauma. 2010;68:E108-E110

[87] Lannes M, Teitelbaum J, Pilar Cortés M, etal. Milrinone and homeostasis to treat cerebral vasospasm associated with subarachnoid hemorrhage: The Montreal neurological hospital protocol. Neurocritical Care. 2012;16:354-362

[88] Lazaridis C, Pradilla G, Nyquist PA, et al. Intra-aortic balloon pump counterpulsation in the setting of subarachnoid hemorrhage, cerebral vasospasm, and neurogenic stress cardiomyopathy. Case report and review of the literature. Neurocritical Care. 2010;13:101-108

[89] Yoneda H, Nakamura T, Shirao S, et al. Multicenter prospective cohort study on volume management after subarachnoid hemorrhage: Hemodynamic changes according to severity of subarachnoid hemorrhage and cerebral vasospasm. Stroke. 2013;44:2155-2161

[90] Hoff RG, Rinkel GJ, Verweij BH, et al. Pulmonary edema and blood volume after aneurysmal subarachnoid hemorrhage: A prospective observational study. Critical Care. 2010;14:R43

[91] Hoff R, Rinkel G, Verweij B, et al. Blood volume measurement to guide fluid therapy after aneurysmal subarachnoid hemorrhage: A prospective controlled study. Stroke. 2009;40:2575-2577

[92] Friedman JA, Pichelmann MA, Piepgras DG, et al. Pulmonary complications of aneurysmal subarachnoid hemorrhage. Neurosurgery. 2003;52:1025-1031

[93] Kahn JM, Caldwell EC, Deem S, et al. Acute lung injury in patients with subarachnoid hemorrhage: Incidence, risk factors, and outcome. Critical Care Medicine. 2006;34:196-202 
[94] Obata Y, Takeda J, Sato Y, et al. A multicenter prospective cohort study of volume management after subarachnoid hemorrhage: Circulatory characteristics of pulmonary edema after subarachnoid hemorrhage. Journal of Neurosurgery. 2016;125(2):254-263

[95] Tagami T, Kuwamoto K, Watanabe A, et al. SAH PiCCO Study Group. Optimal range of global enddiastolic volume for fluid management after aneurysmal subarachnoid hemorrhage: A multicenter prospective cohort study. Critical Care Medicine. 2014;42(6):1348-1356

[96] Dority JS, Oldham JS. Subarachnoid hemorrhage: An update. Anesthesiology Clinics. 2016;34(3):577-600

[97] Spatenkova V, Bradac O, de Lacy P, et al. Dysnatremia as a poor prognostic indicator in patients with acute subarachnoid hemorrhage. Journal of Neurosurgical Sciences. 2017;61(4):371-379

[98] Spatenkova V, Bradac O, Skrabalek P. The impact of a standardized sodium protocol on incidence and outcome of dysnatremias in neurocritical care. Journal of Neurological Surgery Part A: Central European Neurosurgery. 2015;76(4):279-290

[99] Rabinstein AA, Bruder N. Management of hyponatremia and volume contraction. Neurocritical Care. 2011;15:354-360

[100] Audibert G, Steinmann G, de Talancé N, et al. Endocrine response after severe subarachnoid hemorrhage related to sodium and blood volume regulation. Anesthesia and Analgesia. 2009;108:1922-1928 
Section 2

\section{Cerebral Arteriovenous Malformations}





\title{
Cerebral Arteriovenous Malformation from Classification to the Management
}

\author{
Nissar Shaikh, Aisha Al-Kubaisi, Muhammad Mohsin Khan, \\ Adnan Khan, Zia Mahmood, Arshad Chanda, Adel Ganaw, \\ Gamal Al-Ameri, Mostafa Rezk, Moad Ehfeda, \\ Muhammad Zubair, Jazib Hassan and AR Raju Vegesna
}

\begin{abstract}
Cerebral arteriovenous malformations (cAVMs) are the rare neurosurgical emergency. cAVM is an abnormal vascular web, composed of nidus, feeding artery and draining veins. It commonly occurs in the supratentorial area of the brain. The common grading system used in cAVM is Spetzler-Martin grading, which takes into consideration the size of nidus, the location of cAVM and the venous drainage. The cAVMs may develop flow and pressure-related aneurysms, which will increase the morbidity and mortality in these patients. cAVMs vary in size and undergo growth, remodeling and rarely regression. Most of the cAVMs are asymptomatic, but the common presentation are headache, seizure, intracerebral hemorrhage or focal neurological deficit. The cerebral angiography remains the gold standard for the diagnosis of cAVM. Management of the cAVM includes medical therapy, surgical excision, radiosurgery and embolization.
\end{abstract}

Keywords: cerebral angiography, headache, embolization, focal neurological deficit, intracerebral hemorrhage, medical therapy, radiosurgery, seizers, surgical excision, Spetzler-Martin grading

\section{Introduction}

\subsection{Definition}

Cerebral arteriovenous malformations (cAVMs) are the vascular abnormality composed of a complex, tangled web of afferent arteries and draining veins linked by an abnormal dysplastic intervening capillary bed or no capillary bed (Figure 1). The raptured cAVM is a neurosurgical emergency requiring team management approach including neurosurgeons, neuro-interventional radiologist, neuroanaesthesiologist and an intensivist. 

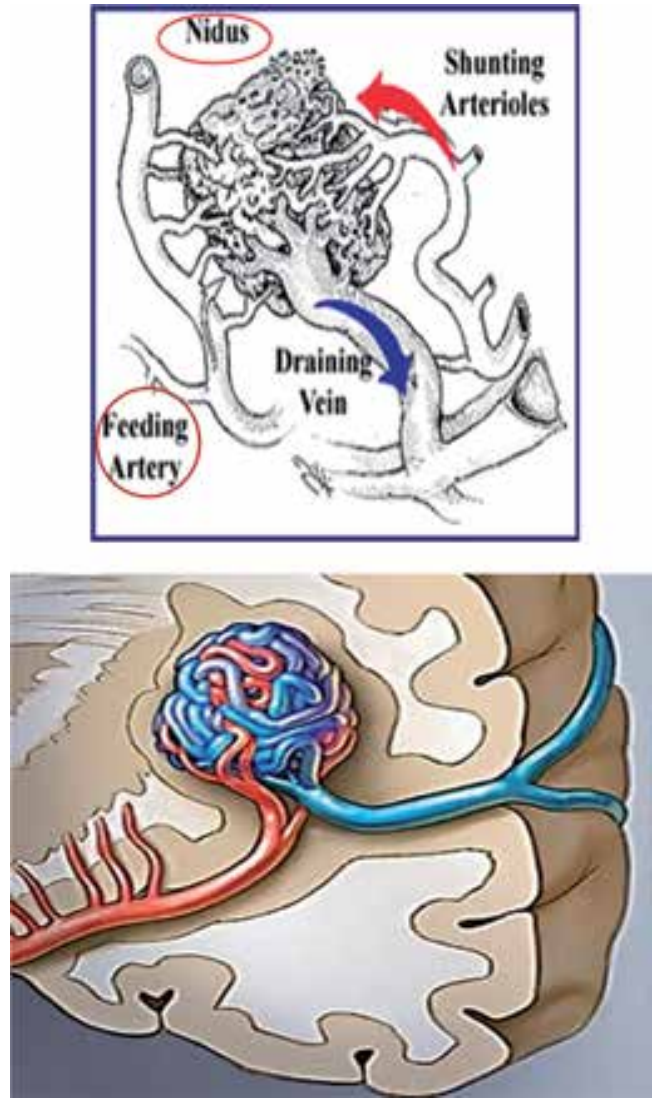

Figure 1.

Cerebral arteriovenous malformation.

\section{Epidemiology}

Cerebral arteriovenous malformations are rare, found in around $0.1 \%$ of the population and one-tenth (1/10th) of the incidence of the brain aneurysms. About $15 \%$ of cAVMs remain asymptomatic based on autopsy series. The majority (90\%) of cAVMs are found in the supratentorial region, the remaining $10 \%$ occur in the posterior fossa. cAVMs commonly occur as single lesions, and in $9 \%$ of the patients, there are multiple AVMs $[1,2]$. cAVM has no gender preference.

cAVM causes subarachnoid hemorrhages in $9 \%$ of the patients. The mean age of presentation is $30-40$ years; $3 \%$ of young patients had stroke due to cAVM. It also causes primary intracerebral hemorrhage in $33 \%$ of young adults [3].

\section{Classifications}

cAVM is classified as follows:

1. Luessenhop-Gennarelli anatomical grading

2. Spetzler-Martin grading (Table 1)/supplementary Spetzler-Martin grading (Table 2) 
Cerebral Arteriovenous Malformation from Classification to the Management

DOI: http://dx.doi.org/10.5772/intechopen.86659

\section{Nataf grading (Table 3)}

\section{Vienna classification (Table 4).}

\begin{tabular}{lc}
\hline Size of cAVM & 1 \\
\hline $0-3 \mathrm{~cm}$ & 2 \\
\hline $3.1-6.0 \mathrm{~cm}$ & 3 \\
\hline$>6 \mathrm{~cm}$ & 0 \\
\hline $\begin{array}{l}\text { Location of cAVM: eloquent areas (sensorimotor, language, visual, thalamus, hypothalamus, internal capsule, } \\
\text { brain stem, cerebellar peduncles and deep cerebellar nuclei) }\end{array}$ & 1 \\
\hline Non-eloquent brain area & 0 \\
\hline Eloquent brain area & 1 \\
\hline Deep venous drainage & \\
\hline Absent & \\
\hline Present & \\
\hline AVM grade $=$ size + eloquence + deep component & \\
\hline
\end{tabular}

Table 1.

Spetzler-Martin grading scale for cAVM.

\begin{tabular}{lcc}
\hline Size of cAVM & \multicolumn{2}{c}{ Patient's age (years) } \\
\hline $0-3 \mathrm{~cm}$ & 1 & $<20$ \\
\hline $3.1-6.0 \mathrm{~cm}$ & 2 & $20-40$ \\
\hline$>6 \mathrm{~cm}$ & 3 & $>40$ \\
\hline Location of cAVM (eloquence area) & & Compactness \\
\hline No & 0 & Yes \\
\hline Yes & 1 & No \\
\hline Venous drainage & & Bleeding \\
\hline Superficial & 0 & Yes \\
\hline Deep & 1 & No \\
\hline
\end{tabular}

Table 2.

Supplementary Spetzler-Martin or Lawton-Young cAVM grading scale.

\begin{tabular}{ll}
\hline Grade I & No risk factor for bleeding \\
\hline Grade Ia & With venous recruitment \\
\hline Grade Ib & Without venous recruitment grade \\
\hline Grade II & Venous stenosis or venous reflux \\
\hline Grade III & Deep venous drainage only \\
\hline Grade IV & Intra- or juxta-nidal aneurysm \\
\hline
\end{tabular}

According to this grading system, there is $13 \%$ of hemorrhage in grade Ia, in grade Ib 38\%, in grade II $48 \%$ and in grade III and IV risk of hemorrhage $90 \%$ [7].

Table 3.

Nataf grading. 


\begin{tabular}{lcccc}
\hline & Gr 0 & Gr I & Gr II & Gr III \\
\hline Feeders & & Pial & Pial + perforators & Perforator \\
\hline Number of feeders & $1-2$ & $>2$ & & \\
\hline Nidus $(\mathrm{cm})$ & $<2$ & $2-4$ & $>4$ & \\
\hline
\end{tabular}

Table 4.

Vienna classification.

\subsection{Luessenhop-Gennarelli grading}

This is the anatomical grading of supratentorial cAVM described in 1977, according to the degrees of surgical difficulty for total obliteration, graded into I-IV. Luessenhop-Gennarelli grading of cAVM is based upon the number of directly participating arteries for which there is a standardized nomenclature [4].

\subsection{Spetzler-Martin (S-M) grading}

In 1986 Spetzler and Martin graded cAVMs according to location, size and deep venous drainage, into the following five grades [5]:

1. Low-grade AVM: Grade I, grade II and grade III

2. High-grade AVM: Grade IV and grade V

3. Inoperable lesions: Grade VI (supplementary Spetzler-Martin (S-M Supp)).

The disadvantages of S-M grading of cAVM are definition of eloquence, lack of consideration for nidus compactness, not useful for posterior fossa AVM, only applicable for surgical outcome and grade III in homogeneity.

Supplementary Spetzler-Martin or Lawton-Young grading scale for cAVM was described in 2010, apart from the risk factors included in S-M grading; this S-M Supp grading system includes factors affecting the patient's outcome, namely, age, bleeding or compactness of the cAVMs (Table 2) [6]. Kim et al. from their cohort multicentre study concluded that S-M Supp grading system works better than the S-M grading for operability decisions and cut-off value for operability is S-M Supp scale of 6. S-M Supp also gives better post-operative neurological outcome predictability than the S-M score.

\subsection{Nataf grading}

In this 4-point grading, five angiographic parameters are used for the determinants of the bleeding risk.

\subsection{Vienna classification}

This classification is helpful in choosing the mode of treatment.

\section{Morphology and type cAVM}

Depending on the type and morphology, cAVM (Figure 2) is divided into the following (Tables 5 and 6 ): 


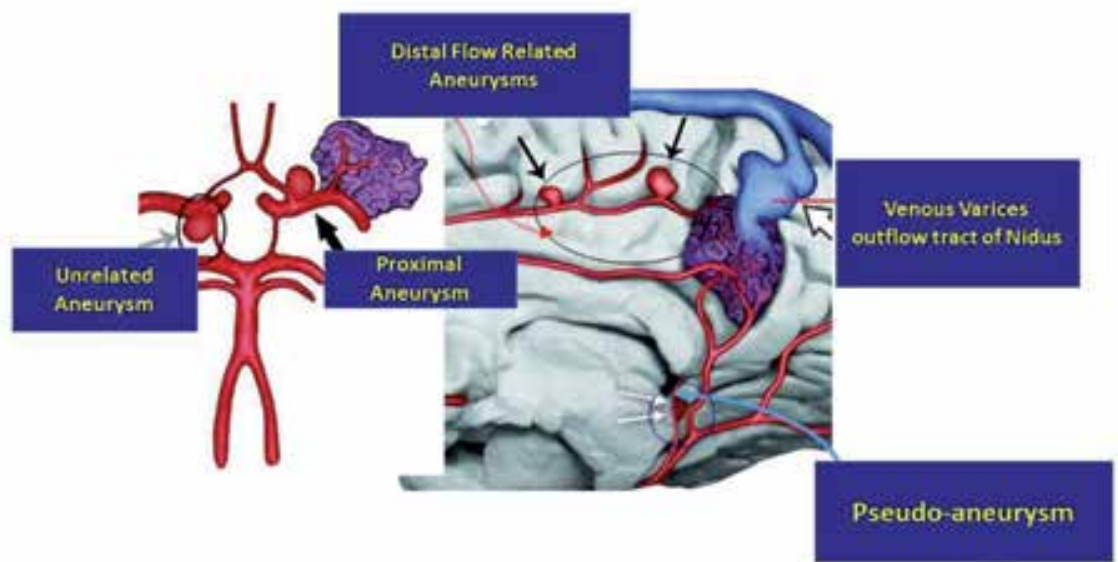

Figure 2.

Details of cAVM and associated aneurysms.

\begin{tabular}{ll}
\hline Cryptic & Absent on angiogram/surgery/pathology \\
\hline Occult & Absent on angiogram/surgery/seen only in pathology examination \\
\hline According to the size of cAVM & \\
\hline Micro-AVM & $<1 \mathrm{~cm}$ \\
\hline Small AVM & $<2.5 \mathrm{~cm}$ \\
\hline Moderate AVM & $2.5-5 \mathrm{~cm}$ \\
\hline Large AVM & $>5 \mathrm{~cm}$ \\
\hline
\end{tabular}

Table 5.

Morphology of cAVM.

\begin{tabular}{llll}
\hline Type & Nature & $\begin{array}{l}\text { Pathological features of the } \\
\text { vessel wall }\end{array}$ & Treatment modality \\
\hline Intranidal & Pseudo-aneurysm & $\begin{array}{l}\text { Disruption of internal elastic } \\
\text { lamina (IEL) }\end{array}$ & Embolization \\
\hline $\begin{array}{l}\text { Distal } \\
\text { flow-related }\end{array}$ & $\begin{array}{l}\text { Saccular aneurysm, } \\
\text { arising from the } \\
\text { arterial trunk }\end{array}$ & $\begin{array}{l}\text { Disruption of IEL, deficiency in } \\
\text { the smooth muscle layer }\end{array}$ & $\begin{array}{l}\text { The aneurysm and the } \\
\text { parent artery were occluded } \\
\text { with Onyx at the same } \\
\text { sitting }\end{array}$ \\
\hline $\begin{array}{l}\text { Proximal } \\
\text { flow-related }\end{array}$ & Segmental ectasia & $\begin{array}{l}\text { Stretched/fragmented IEL: no } \\
\text { luminal thrombus }\end{array}$ & $\begin{array}{l}\text { Coiling for symptomatic } \\
\text { lesion }\end{array}$ \\
\hline Unrelated & $\begin{array}{l}\text { Saccular, arising } \\
\text { from the Willis } \\
\text { circle or a site of } \\
\text { bifurcation }\end{array}$ & Disruption of the IEL & $\begin{array}{l}\text { Simply coiling or stent- } \\
\text { assisted coiling }\end{array}$ \\
& & & \\
\hline
\end{tabular}

Table 6.

Classification of aneurysm with cAVM [8].

\section{Pathophysiology}

The pathogenesis of cAVMs is not well understood; they are considered congenital and can undergo growth, remodeling or regression over the period. The 
size of cAVMs varies widely, and cAVM can remain clinically silent for decades. In cAVM blood flows directly between them without capillary bed and not intervening neural parenchyma in nidus. cAVMs is a high-flow and high-pressure system. Particularly the small AVMs are having significantly higher pressure in the feeding arteries; hence the small AVMs are more lethal than the larger ones and bleed more frequently.

Familial cAVMs have been reported, but it is not clear whether these are coincidental or indicate a familial occurrence. Angio-architecture of cAVMs is direct arterial to venous connections without an intervening capillary network. Both the arterial supply and the venous drainage may be by single or multiple vessels. The high-flow arteriovenous communication potentiates a variety of flow-related phenomena such as the development of afferent and efferent pedicle aneurysms, which occur in $20-25 \%$ of patients, and arterialization of the venous limb. Aneurysms can be a source of bleeding with cAVMs and may indicate a grave prognosis [9]. Abnormal flow and a vascular steal phenomenon have been suggested to underlie some clinical symptoms associated with cAVMs [10]. Histopathological studies demonstrate areas of chronic ischemia and gliosis in the region of the cAVM malformation. Angio-structure of cAVM is typically composed of arterial feeder, nidus, draining vein and intervening gliotic neural parenchyma. The arterial feeder can be single or multiple originating from pial or perforating or dural arteries, and it can be a direct feeder supply as terminal branch or indirect feeder supply enpassage. Nidus is a compact tangle of dysplastic, thin-walled vessels of varied length connecting feeding arteries to draining veins. cAVM nidus can either be globular or conical in shape and may be compact or diffuse. Within the nidus, arterial blood is shunted directly into draining veins without passage through a normal, highresistance arteriolar-capillary network.

\section{Clinical presentation}

cAVMs commonly present in younger age group ranging from 10 to 40 years. The clinical presentation of cAVM depends on the patient's age, size, location and vascular features of the cAVM. These patients commonly present with headache or neurological deficit or convulsions or intracerebral hemorrhage.

\subsection{Headache}

Around $0.2 \%$ of patients with headache and normal neurologic examinations were associated with cAVM [11]. Usually headache associated with cAVM is incidental, without any specifications. Headaches are the presenting symptom in approximately $15 \%$ of patients without evidence of rupture. Headache can be characterized as similar to migraines with lateralization to one side.

\subsection{Focal neurologic deficit}

This is quite an unusual presentation of cAVM. A vascular steal syndrome is hypothesized to be the cause for focal neurologic deficit [1].

\subsection{Seizure (convulsions)}

About $11-33 \%$ of cAVM patients present with convulsions, and patients with cortical-located, large, multiple and superficial-draining cAVMs present with convulsions [12]. Convulsions are focal, simple or partial complex with secondary 


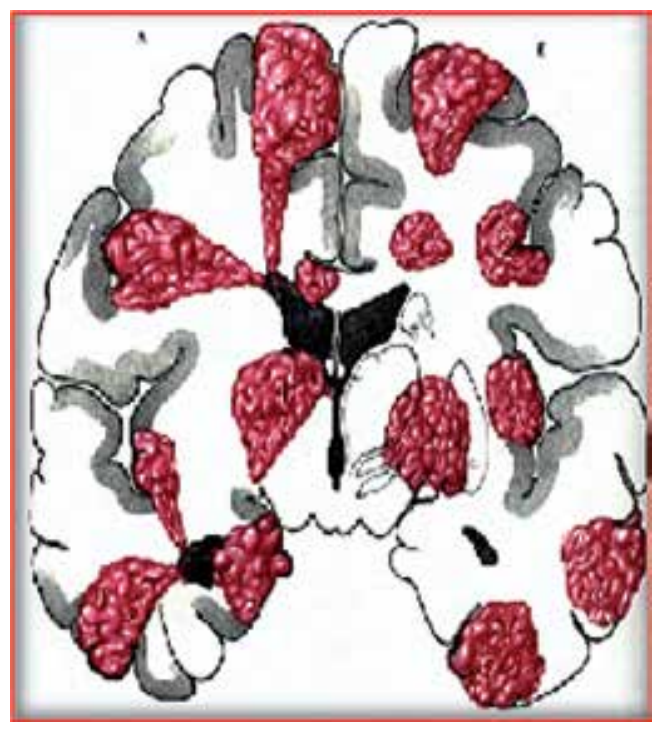

Figure 3.

Graphic representation of intracerebral hemorrhage.

generalization. cAVM-related seizures may occur due to the overt intracranial hemorrhage, from the hemosiderin deposition, or secondary to venous hypertension, ischemia following steal. The cAVM location influences the seizure type and semiology. The 5-year seizure risk for cAVMs is found to be $8 \%$ [13].

\subsection{Intracerebral hemorrhage}

About $41-79 \%$ of cAVM patients present with intracerebral hemorrhage (ICH) (Figure 3). Children more frequently present with intracerebral hemorrhage than adults [14]; $23 \%$ of patients with ICH or focal neurologic deficits had a 5-year risk of seizures. Hemorrhage can be parenchymal, intraventricular or subarachnoid.

Patients with cAVMs can have ICH on initial presentation. The deep venous drainage and deep brain location of cAVM had higher incidence of $\mathrm{ICH}$, a recurrent AVM hemorrhage of $12 \%$ over an average with an average rebleeding rate of $7 \%$ annually [15]. Pregnancy is not a risk factor for hemorrhage from a cAVM, but the data are not definitive [16]; cAVM hemorrhage rate in pregnant women was not significantly different compared with the rate for non-pregnant women [17].

\section{Diagnosis}

Imaging studies are essential for the establishment of the diagnosis of $\mathrm{cAVM}$, to make a pre-therapeutic evaluation of the cAVM to help in decision-making, to treat the AVM as a sole therapy or a multidisciplinary approach. It also helps in posttherapeutic evaluation of cAVM.

\subsection{Computerized tomography (CT) of the brain}

The CT of the brain is the first imaging procedure used to rule out hemorrhage. cAVM is suspected in young patients with lobar hematoma without significant oedema and calcifications. The parenchymal calcifications are found in $20 \%$ of cases, related to intravascular thrombosis or evolution of an old hematoma. 


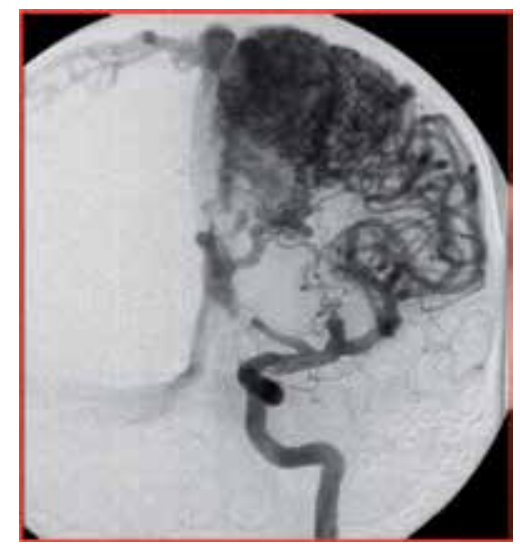

Figure 4.

Cerebral angiogram showing $c A V M$.

The compression of the nidus by hematoma precludes CT diagnosis of cAVM in patients with acute intracerebral hemorrhage; in these patients CT angiography and magnetic resonance angiography are essential.

\subsection{Magnetic resonance imaging (MRI) of the brain}

MRI of the brain is highly sensitive for the location of the cAVM nidus and an associated draining vein; it also has a unique sensitivity in demonstrating remote bleeding related to these lesions. MRI of the brain is particularly valuable in follow-up patients after treatment. After radiosurgery MRI is important for the regression of the nidal volume. Whereas the MR angiography techniques can characterize the venous drainage and other vascular features, the surrounding tissue that is subjected to the radiation field can be accurately monitored for post-therapy oedema or radiation necrosis [18].

\subsection{Cerebral angiography}

It is the gold standard for the diagnosis, treatment planning and follow-up after treatment of cAVMs. The anatomical and physiological information of the cAVM nidus, its relationship to surrounding vessels and localization of the draining or efferent portion of the cAVM are readily obtained with this cerebral angiography. It will also help in demonstration of associated aneurysm suggesting a lesion at higher risk for subsequent hemorrhage. The contrast transit times provide additional useful information regarding (Figure 4).

\section{Treatment}

The treatment plans of cAVM are most appropriately made by a multidisciplinary team of experienced clinicians with the consideration of size, location and vascular features of the AVM [19]. While making the decision, whether a patient should undergo AVM treatment, several factors have to be considered including the short- and long-term risk of hemorrhage, feasibility, associated aneurysm, patient's age, risks of intervention, availability of interventional radiologist and size and compactness of the nidus. The short-term and long-term hemorrhage risk is associated with a history of raptured cAVM, patient age, AVM location, size and vascular morphological features. 
The three types of modalities of treatment are microsurgery and excision of cAVM, embolization and stereotactic radiosurgery. It's frequent that these modalities are used in combination to achieve the complete treatment. In a systemic review, it was found that available treatment modalities were associated with risks including $5.1-7.4 \%$ of the median rate of permanent neurologic complications or death and incomplete efficacy of $13-96 \%$ [20]. Medical management of cAVM is considered when the patient has suffered a devastating neurological deficit; cAVM is very extensive, located deep in the brain, with blood supply primarily from deep perforating vessels, which are not amenable to endovascular or radiosurgical therapy, advanced age and poorly controlled comorbidities. We will discuss these modalities in the following subheadings.

\subsection{Microsurgical cAVM excision}

Microsurgical AVM excision is the most effective treatment of cAVM; it has the longest history of use for the definitive treatment of cAVMs and offers the best chance of immediate cure in patients at high risk of hemorrhage [19]. Factors associated with increased surgical risk are large size, deep venous drainage, deep location, diffuse nidus and feeders from deep perforating system. Surgeons should follow few basic surgical principles, cAVM excision should be an elective procedure, irrespective of the ruptured or unraptured cAVMs, preoperative steroids, anticonvulsants and antibiotics to be started, a wider craniotomy and dural opening, if the nidus is below the surface, an arterialized draining vein (red vein) can be followed to the AVM, localization may be assisted with ultrasound or frameless stereotaxic, dura should be open carefully, circumferential dissection of the nidus layer by layer, at least one major draining vein should be preserved till end of dissection, at end of dissection this vein becomes bluish proving that feeders have been eliminated, securing the individual vessels can be done with low power bipolar coagulation, but care should be taken as the excessive bipolar coagulation usage can cause retraction into eloquent tissue leading to the significant neurological morbidity. The complete AVM excision should be documented by post-op angiogram. Excision of the whole nidus is necessary to protect against rebleeding. If cAVM are high-flow, preoperative embolization should be considered, and cAVM supplies with multiple vascular territories may require a surgical staging. If cAVM are ectatic, high blood flow and irregular vessels will help in the identification. The feeding arteries (Table 7) can be distinguished from draining veins not by sight or look, as the veins will have arterial blood but by noting if the distal vessel collapses with gentle occlusion of the vein. The surgeon should always secure the feeding artery as close to the nidus as possible to ensure that this is not a passage artery feeding the normal brain, unless there is definite evidence on an angiogram that an artery is a direct nidus feeder.

\begin{tabular}{ll}
\hline Size & Vein dilates considerably, the vessel $>4 \mathrm{~mm}$ is a vein unless proven otherwise \\
\hline Color & $\begin{array}{l}\text { Due to oxygenated blood, both appear red, but on careful view under illumining microscopy, } \\
\text { the vein appears bright red due to thin muscular layer, and the artery appears light pink }\end{array}$ \\
\hline $\begin{array}{l}\text { Flow on } \\
\text { occlusion }\end{array}$ & Upon gentle compression of the vein, it will collapse distally unlike the artery \\
\hline $\begin{array}{l}\text { Vascular } \\
\text { course }\end{array}$ & Arteries dive into sulci and fissure when veins rise to the surface towards sinuses \\
\hline
\end{tabular}

Table 7.

Differentiation of the artery from the vein in cAVM. 


\subsection{The morbidity and mortality of microsurgical excision of cAVMs}

According to Castel and Kantor, morbidity and mortality after microsurgical excision of cAVM vary from 1.5 to $18.7 \%$ and from 0 to $15 \%$, respectively [21]. Morbidity is high (20\%) in deep-seated cAVM compared to the smaller cAVM with morbidity of up to $9 \%$ (Tables 8 and 9 ).

\subsection{Stereotactic radiosurgery for cAVMs}

High-energy beams of photons or protons to a defined volume containing cAVM nidus are stereotactically focused, and it induces progressive thrombosis of lesions by fibrointimal hyperplasia and subsequent luminal obliteration (Figure 5). The time between treatment and obliteration is referred to as the latency period, and it varies from 1 to 3 years. One has to wait for radiosurgery till the hematoma resolves. The factors influencing the obliteration rate of cAVM are cAVM size, target determination, angio-architecture and haemodynamics, cAVM location (hemispheric better) and radiation dosage. Stereotactic radiosurgery for cAVMs has disadvantages of the risk of hemorrhage in latency period and individual variability to radiosensitivity and neurological deficit.

The neurological deficit after radiosurgery is observed in $5.37 \%$ of the cases, and it was permanent in $1.46 \%$. The mortality was $1.6 \%$, and it is mainly due to the bleeding in the latency period [24].

The other complications after radiosurgery are radiation necrosis, which causes new neurologic deficits and seizures, and around $8 \%$ of the patients develop the parenchymal lesions, cranial nerve deficits, seizures, headaches and cyst formation [25]. The incidence of complications is related to cAVM location and the volume treated. Thalamic, basal ganglionic and brainstem locations are prone to development of deficits after radiosurgery [26]. The risk of complications is also related to

\begin{tabular}{l}
\hline Intraoperative rupture \\
\hline Post-retraction oedema \\
\hline Seizures \\
\hline Retrograde thrombosis of feeding vessels \\
\hline Resection of eloquent brain \\
\hline Normal perfusion pressure breakthrough (haemorrhage in normal brain tissue surrounding the lesion) \\
\hline
\end{tabular}

Table 8.

Complication of microvascular surgery.

\begin{tabular}{lc}
\hline S-M grading & Patient's outcome \\
\hline Grade I & $92-100 \%$ \\
\hline Grade II & $95 \%$ \\
\hline Grade III & $88 \%$ \\
\hline Grade IV & $73 \%$ \\
\hline Grade V & $57 \%$ \\
\hline (Heros RC, Korusure K, Diebuild PM. Surgical excision if cerebral arteriovenous malformations. Neurosurgery. \\
1990;26:570-577).
\end{tabular}

Table 9.

Spetzler-Martin grading and patient's outcome after microsurgery [22, 23]. 


\section{cAVM Obliteration rate (\%) Accoding to Patients Age (Years)}

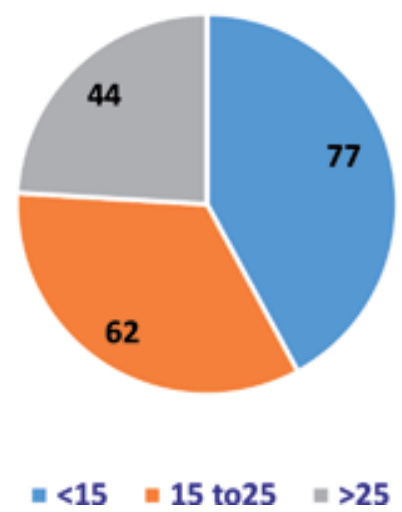

Figure 5.

Percentage of cAVM obliteration according to the nidus size $(\mathrm{mm})$ by radiosurgery [25].

the radiation dose directed to the surrounding tissue and is increased in large brain AVMs that require larger treatment volumes [27]. Repeated radiosurgery is associated with increased complications [28].

\subsection{Embolization}

A rapidly evolving technique uses embolic agents such as silk, Histoacryl with Lipiodol and Onyx and delivery systems such as balloons with calibrated leaks, flow-guided micro-catheters and over-the-wire micro-catheters (Figure 6). It was used initially as an adjunct to microsurgery or radiosurgery; nowadays in selected cases, embolization is a sole treatment modality. By embolization less than $5 \%$ of cAVMs are cured.

Embolization can be an effective adjunct to radiosurgery and surgery. Embolization prior to radiosurgery is used to reduce the nidus size of large brain AVMs as the large AVMs have a lower cure rate with radiosurgery alone. Embolization prior to surgery is employed to reduce blood loss and to occlude

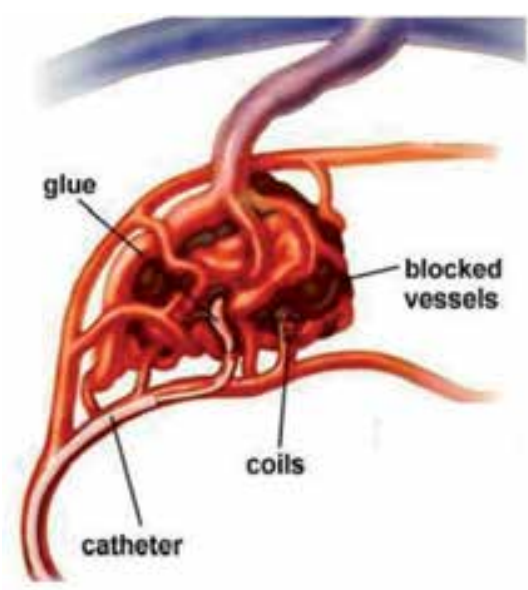

Figure 6.

Embolization process. 
vessels that may be difficult to control during surgery [29]. Pre-embolization steroids and anti-epileptic medications should be started; the procedure is done under general anesthesia. The aims of cAVM embolization could be curative or palliative, and it could be partial prior to the radio- or microscopic surgery. Careful analyses of angiographic information including size, eloquent location, deep versus superficial venous drainage and vascular anatomy/number of feeders are considered for the suitability for embolization of cAVMs.

The cAVM embolization can be complicated into partial occlusion, intracranial hemorrhage, brain ischemia, glueing of micro-catheter or perfusion pressure breakthrough syndrome. Perfusion pressure breakthrough syndrome occurs in approximately $5 \%$ of the patients, thought to be related to the loss of autoregulation causing oedema or hemorrhages in the surrounding normal brain tissues [30]. The successful cAVM embolization occurs in $10-40 \%$ of the patients and may have to be done in the multiple settings.

To summarize the treatment of cAVMs, the following factors have to be considered: the patient's age and anatomic and vascular morphology of the cAVM.

\subsection{Patient's age}

It is an important factor in the decision to treat cAVMs. Those with a longer life expectancy will accrue a higher lifetime risk of hemorrhage. Accordingly therapy is recommended for children and young adults, whereas the older cAVM patients with shorter life expectancies may be managed more conservatively. The cumulative risk of hemorrhage in cAVM patients can be calculated by the formula [31]: Lifetime risk of hemorrhage $=1-(1-\mathrm{P}) \times \mathrm{N}$ ( $\mathrm{N}$ is the expected years of life remaining, and $\mathrm{P}$ is the annual probability of hemorrhage). Another simpler formula is as follows: [32] Lifetime risk of hemorrhage $=105$ - patient's age in years.

\section{6 cAVM location}

cAVM in eloquent brain territories presents a challenge for risk assessment. Significant clinical morbidity is likely to result if a surgical complication occurs as well as if the cAVM ruptures. Such patients may be more likely to be considered for radiosurgery.

\subsection{Deep venous drainage}

Deep venous drainage is a risk factor for surgical complications as well as for $A V M$ rupture. If the lesion is otherwise amenable for treatment, radiosurgery may be the best option.

\subsection{The cAVM size}

Larger lesions are more difficult to treat but are not clearly at higher risk of bleeding than smaller lesions. cAVMs greater than $6 \mathrm{~cm}$ are managed conservatively. In patients with large lesions, the endovascular treatments may be useful to reduce the size of the aneurysm, if the vascular anatomy is determined to be amenable to this approach.

\subsection{Associated aneurysm}

The treatment of aneurysms associated with AVMs varies depending on aneurysm location and diameter [33]. When believed to be the source of hemorrhage, 
Cerebral Arteriovenous Malformation from Classification to the Management DOI: http://dx.doi.org/10.5772/intechopen.86659

\begin{tabular}{lccc}
\hline & Microsurgery & Radiosurgery & Embolization \\
\hline Low procedural invasivity & 1 & 3 & 2 \\
\hline Occlusion capacity & 3 & 2 & $1-2$ \\
\hline Speed of efficacy & 3 & 1 & $2-3$ \\
\hline Long-term reliability & 3 & 3 & $2-3$ \\
\hline Independent from size & 2 & 1 & 3 \\
\hline Independent from brain functionality & 1 & 2 & 3 \\
\hline Independent from angio-architecture & 2 & 2 & 1 \\
\hline Independent from flow & 3 & & \\
\hline 1: no/low, 2: medium, and 3: yes/high. & & & \\
\hline
\end{tabular}

Table 10.

Factors for selecting the modalities of therapy in cAVM patients [34].

aneurysms are generally treated with surgery or endovascular therapy, depending on their location and size, according to the expertise of the available experienced clinicians. Aneurysms associated with unruptured cAVMs do not necessarily require treatment, depending on their size and other anatomic features.

Spetzler-Martin grading scale: Spetzler-Martin grading scale classifies surgical risk of removing cAVM according to its size, location and the presence of deep venous drainage. According to S-M grading, surgery is the modality of choice for grade I and II lesions, with radiosurgery as an alternative for small lesions based on location or other vascular anatomic features, while grade IV and V lesions are generally managed conservatively. S-M Supp grading gives a cut-off scale of 6 for operability of cAVM, and it is also a better predictor for post-cAVM excision neurological outcome. Hafez et al. [34] nicely described that apart from the S-M Supp grading, perforates play an important role in cAVM patient's outcome (Table 10) [35].

\section{Conclusion}

Cerebral arteriovenous malformations are rare and 10 times less frequent than the cerebral aneurysms. cAVMs are rare in the posterior fossa and account for $33 \%$ of intracerebral hemorrhage in young patients. They are classified by four different ways, but the commonly used classifications are Spetzler-Martin grading and supplementary Spetzler-Martin grading, as they also help in deciding the therapeutic approach. But it is not much useful in dealing the posterior fossa cAVMs. cAVMs can complicate into the formation of flow-related aneurysms and, according to their locations in relation to the cAVM, are classified into intranidal, distal, proximal or unrelated aneurysms. Angio-structure of cAVM is the direct connection between arterial and venous circulations without any intervening capillary network, and this makes cAVM high in flow and pressure structure at the risk of rapture.

The common presentation of cAVM is with intracerebral hemorrhage, with convulsions and rarely with focal neurological deficit. The most commonly used imaging modality for the diagnosis of cAVMs is computerized tomography. MRI scan has a unique sensitivity for the detection of remote hemorrhage, and conventional catheter cerebral angiography remained a gold standard in diagnosis of cAVMs. Multidisciplinary team approach is the best for management of cAVM patients. The surgical excision of cAVM gives definitive therapy in cAVM patients, radiosurgery is curative in selected cAVM patients and cerebral angiographic embolization is 
rarely a sole therapy and usually in combination with surgical therapy. The medical therapies of cAVM are anticonvulsants and steroids. While taking a therapeutic decision for cAVMs, it is of vital importance to consider the patient's age, location, deep venous drainage, size of cAVMs and the presence of the associated aneurysms. The associated aneurysms must be secured before the interventions for cAVMs.

\section{Author details}

Nissar Shaikh ${ }^{1 *}$, Aisha Al-Kubaisi², Muhammad Mohsin Khan ${ }^{2}$, Adnan Khan², Zia Mahmood ${ }^{1}$, Arshad Chanda ${ }^{1}$, Adel Ganaw ${ }^{1}$, Gamal Al-Ameri ${ }^{1}$, Mostafa Rezk ${ }^{1}$, Moad Ehfeda ${ }^{1}$, Muhammad Zubair ${ }^{1}$, Jazib Hassan ${ }^{1}$ and AR Raju Vegesna ${ }^{1}$

1 Surgical Intensive Care, Hamad Medical Corporation, Doha, Qatar

2 Department of Neurosciences, Hamad Medical Corporation, Doha, Qatar

*Address all correspondence to: nissatfirdous99@gmail.com

\section{IntechOpen}

(C) 2019 The Author(s). Licensee IntechOpen. This chapter is distributed under the terms of the Creative Commons Attribution License (http://creativecommons.org/licenses/ by/3.0), which permits unrestricted use, distribution, and reproduction in any medium, provided the original work is properly cited. (cc) BY 


\section{References}

[1] Mohr JP, Kejda-Scharler J, PileSpellman J. Diagnosis and treatment of arteriovenous malformations. Current Neurology and Neuroscience Reports. 2013;13(2):324

[2] Willinsky RA, Lasjaunias P, Terbrugge K, Burrows P. Multiple cerebral arteriovenous malformations (AVMs). Review of our experience from 203 patients with cerebral vascular lesions. Neuroradiology. 1990;32:207

[3] Diringer MN, Bleck TP, Claude Hemphill J 3rd, et al. Critical care management of patients following aneurysmal subarachnoid hemorrhage: Recommendations from the Neurocritical Care Society's Multidisciplinary Consensus Conference. Neurocritical Care. 2011;15:211

[4] Luessenhop AJ, Gennarelli TA. Anatomical grading of supratentorial arteriovenous malformations for determining operability. Neurosurgery.

1977;1(1):30-35

[5] Spetzler RF, Martin NA. A proposed grading system for arteriovenous malformations. Journal of Neurosurgery. 1986;65(4):476-483

[6] Kim H, Abla AA, Nelson J, McCulloch CE, Bervini D, Morgan MK, et al. Validation of the supplemented Spetzler-Martin grading system for brain arteriovenous malformations in a multicentre cohort of 1009 surgical patients. Neurosurgery. 2015;76(1):25-33

[7] Nataf F, Schlienger M, Bayram M, Ghossoub M, George B, Roux FX. Microsurgery or radiosurgery for cerebral arteriovenous malformations? A study of two paired series. Neurosurgery. 2007;61:39-49
[8] Lv X, Wu Z, He H, Ge H, Li Y. Proposal of classification of aneurysms coexisting with AVM and possible treatment strategies. Turk Neurosurgical. 2016;26(2):229-233. DOI: 10.5137/1019-5149.JTN.8600-13.1

[9] Perata HJ, Tomsick TA, Tew JM Jr. Feeding artery pedicle aneurysms: Association with parenchymal hemorrhage and arteriovenous malformation in the brain. Journal of Neurosurgery. 1994;80:631

[10] Moftakhar P, Hauptman JS, Malkasian D, Martin NA. Cerebral arteriovenous malformations. Part 2: Physiology. Neurosurgical Focus. 2009;26:E11

[11] Evans RW. Diagnostic testing for the evaluation of headaches. Neurologic Clinics. 1996;14:1

[12] Garcin B, Houdart E, Porcher R, et al. Epileptic seizures at initial presentation in patients with brain arteriovenous malformation. Neurology. 2012;78:626

[13] Josephson CB, Leach JP, Duncan R, et al. Seizure risk from cavernous or arteriovenous malformations: Prospective population-based study. Neurology. 2011;76:1548

[14] Fullerton HJ, Achrol AS, Johnston SC, et al. Long-term hemorrhage risk in children versus adults with brain arteriovenous malformations. Stroke. 2005;36:209

[15] Choi JH, Mast H, Sciacca RR, et al. Clinical outcome after first and recurrent haemorrhage in patients with untreated brain arteriovenous malformation. Stroke. 2006;37:1243

[16] Brown RD Jr, Flemming KD, Meyer FB, et al. Natural history, evaluation, and management of intracranial vascular malformations. Mayo Clinic Proceedings. 2005;80:269 
[17] Horton JC, Chambers WA, Lyons SL, et al. Pregnancy and the risk of hemorrhage from cerebral arteriovenous malformations. Neurosurgery. 1990;27:867

[18] Saleh RS, Singhal A, Lohan D, et al. Assessment of cerebral arteriovenous malformations with high temporal and spatial resolution contrast-enhanced magnetic resonance angiography: A review from protocol to clinical application. Topics in Magnetic Resonance Imaging. 2008;19:251

[19] Barr JC, Ogilvy CS. Selection of treatment modalities or observation of arteriovenous malformations. Neurosurgery Clinics of North America. 2012;23:63

[20] van Beijnum J, van der Worp HB, Buis DR, et al. Treatment of brain arteriovenous malformations: A systematic review and meta-analysis. Journal of the American Medical Association. 2011;2011:306

[21] Castel JP, Kantor G. Postoperative morbidity and mortality after microsurgical exclusion of cerebral arteriovenous malformations. Current data and analysis of recent literature. Neuro-Chirurgie. 2001;47:369-383

[22] Sasaki T, Kurita H, Saito I, Kawamoto S, Nemoto S, Terahara A, et al. Arteriovenous malformations in the basal ganglia and thalamus: Management and results in 101 cases. Journal of Neurosurgery. 1998;88(2):285-292

[23] Schaller C, Schramm J, Haun D. Significance of factors contributing to surgical complications and to late outcome after elective surgery of cerebral arteriovenous malformations. Journal of Neurology, Neurosurgery and Psychiatry. 1998;65:547-554

[24] Nataf F et al. Results of series of 705 AVM treated by radiosurgery. Neuro-Chirurgie. 2001;47:268-282
[25] Flickinger JC, Kondziolka D, Lunsford LD, et al. A multi-institutional analysis of complication outcomes after arteriovenous malformation radiosurgery. International Journal of Radiation Oncology, Biology, Physics. 1999;44:67

[26] Pollock BE, Gorman DA, Brown PD. Radiosurgery for arteriovenous malformations of the basal ganglia, thalamus, and brainstem. Journal of Neurosurgery. 2004;100:210

[27] Miyawaki L, Dowd C, Wara W, et al. Five year results of LINAC radiosurgery for arteriovenous malformations: Outcome for large AVMS. International Journal of Radiation Oncology, Biology, Physics. 1999;44:1089

[28] Buis DR, Meijer OW, van den Berg $\mathrm{R}$, et al. Clinical outcome after repeated radiosurgery for brain arteriovenous malformations. Radiotherapy and Oncology. 2010;95:250

[29] Krings T, Hans FJ, Geibprasert S, Terbrugge K. Partial "targeted" embolization of brain arteriovenous malformations. European Radiology. 2010;20:2723

[30] Young WL, Kader A, et al. Pressure autoregulation is intact after arteriovenous malformation resection. Neurosurgery. 1993;32:491-497

[31] Kondziolka D, McLaughlin MR, Kestle JR. Simple risk predictions for arteriovenous malformation hemorrhage. Neurosurgery. 1995; 37:851

[32] Brown RD Jr. Simple risk predictions for arteriovenous malformation hemorrhage. Neurosurgery. 2000;46:1024

[33] Friedlander RM. Clinical practice. Arteriovenous malformations of the brain. The New England Journal of Medicine. 2007;356:2704 
Cerebral Arteriovenous Malformation from Classification to the Management DOI: http://dx.doi.org/10.5772/intechopen.86659

[34] Hafez A, Koroknay-Pál P, Oulasvirta E, Elseoud AA, Lawton MT, Niemelä $\mathrm{M}$, et al. The application of the novel grading scale (Lawton-Young Grading System) to predict the outcome of brain arteriovenous malformation. Neurosurgery. 2019;84(2):529-536

[35] Ogilvy CS, Stieg PE, Awad I, et al. AHA Scientific Statement: Recommendations for the management of intracranial arteriovenous malformations: A statement for healthcare professionals from a special writing group of the Stroke Council, American Stroke Association. Stroke. 2001;32:1458 



\title{
Chapter 4
}

\section{Endovascular Treatment of Cerebral Arteriovenous Malformations}

\author{
Cagin Senturk
}

\begin{abstract}
Arteriovenous malformations (AVM) are vascular malformations composed of a network of abnormal vessels connecting directly between the artery and vein without an intervening capillary bed. Cerebral arteriovenous malformations have an incidence of $0.8-1.3$ per 100.000 person years. Clinical symptoms include intracranial hemorrhage, seizure, headache, and focal neurological deficit. Annual mortality rate associated with hemorrhage is $1-5 \%$, and $10-30 \%$ of survivors will have disability. Treatment options for cerebral AVMs include open surgery, embolization, and radiosurgery. Depending on the grade and angioarchitectural characteristics, a combination of these modalities can be used. Endovascular treatment can be performed for size and grade reduction, presurgical devascularization, size reduction before radiosurgery, targeted embolization, and as stand-alone treatment for cure. Targeted embolization can address intranidal or flow-related aneurysms and high flow arteriovenous shunts. Complications of the endovascular treatment include hemorrhage related to vessel perforation or normal pressure breakthrough phenomenon, ischemia, microcatheter retention, and other general complications associated with angiographic procedures. Mortality associated with endovascular treatment is less than $2 \%$ and permanent neurological deficit can be seen up to $2-8.9 \%$ of cases. New endovascular techniques include balloon-assisted embolization, transvenous embolization, and double microcatheter techniques like pressure cooker technique.
\end{abstract}

Keywords: cerebral arteriovenous malformation, intracranial hemorrhage, endovascular, embolization

\section{Introduction}

Arteriovenous malformations (AVM) are vascular malformations composed of a network of abnormal vessels connecting directly between the artery and vein without an intervening capillary bed. AVMs are thought to be congenital lesions originated from persistence of primitive arteriovenous connections [1].

\subsection{Epidemiology}

According to epidemiological studies, including Netherland Antilles [2], Olmsted county Minnesota [3], and New York islands [4], incidence of cerebral 
arteriovenous malformations is between 0.8 and 1.3 per 100.000 person years. In addition to sporadic cases, brain AVMs can be associated with syndromes including hereditary hemorrhagic telangiectasia, Wyburn-Mason syndrome, and SturgeWeber syndrome $[5,6]$.

\subsection{Clinical presentation}

Symptomatic brain AVMs may present with intracranial hemorrhage (50\%), seizure (33\%), headache (16\%), or focal neurologic deficit (6\%) [7]. Annual risk of bleeding of due to brain AVMs is approximately 2-4\%. Risk factors increasing the odds of bleeding include prior history of intracranial bleeding due to AVM, deep location, exclusive deep venous drainage and single draining vein, intranidal aneurysm, and high intranidal pressure [7-9]. When there are three factors, risk may increase up to $34 \%$. In patients presenting with hemorrhage, rebleeding risk in the first year is approximately $32 \%$ that decrease to $11 \%$ in subsequent years [10]. Annual mortality rate is approximately $1.5 \%$ and $10-30 \%$ of survivors have long-term disability. Neurological disability is more common in ruptured AVMs compared to aneurysm rupture due to higher likelihood of a lobar hematoma [11]. Although there is conflictive data, smaller AVMs have a higher tendency to present with hemorrhage. Spetzler et al. [12] found that $82 \%$ of smaller AVMs $(<3 \mathrm{~cm})$ present with hemorrhage compared to $21 \%$ of hemorrhage seen in larger AVMs $(>6 \mathrm{~cm})$. Ondra et al. [13] published a series of 160 symptomatic untreated AVM cases and found that $23 \%$ of the patients died during a mean follow-up of 23.7 years.

\subsection{Therapeutic strategies}

AVM treatment includes medical management, surgical, endovascular, and radiosurgical modalities. ARUBA, largest multicenter randomized trial to date, showed that medical management alone is superior to interventional therapy for the prevention of death and stroke in patients with unruptured AVMs [14, 15]. However, the follow-up period in this study was only 33 months, and 5-year follow-up results that will prove whether these results are persistent are yet to be published. Nevertheless, ruptured AVMs, unruptured AVMs with significant risk factors, and some symptomatic AVMs in young patients must be treated. These treatment modalities can be used as stand-alone treatment for cure, or a combination of different techniques can be used to increase the efficiency and minimize the risks associated with treatment. Although there is still no consensus on the ideal treatment, every case is evaluated specifically for its rupture risk and risks associated with its treatment. The most common grading system used to stratify the risks of surgical treatment of AVMs is the Spetzler-Martin system [16]. This system classifies the AVMs according to size, location, and venous drainage. Larger lesions, AVMs with deep venous drainage, and lesions in eloquent locations have higher surgical risk. Eloquent locations include sensorimotor cortex, visual cortex, thalamus, internal capsule, brainstem, cerebellar peduncles, and deep cerebellar nuclei. Deep venous drainage sites are straight sinus, internal cerebral veins, basal veins of Rosenthal, and precentral cerebellar veins. Spetzler and Ponce proposed a modified version of the grading system in 2011 [17].

AVMs larger than $3 \mathrm{~cm}$ in a non-eloquent and superficial location can be safely treated with embolization followed by surgery. AVMs larger than $3 \mathrm{~cm}$ with a deep or eloquent location can be embolized and then radiosurgery can be used for the ultimate cure. Smaller lesions $(<3 \mathrm{~cm})$ can be safely treated with surgery alone or 
radiosurgery can be used as stand-alone treatment in case of eloquent or deep location. For ruptured small deeply located surgically inaccessible AVMs, embolization can be used as a stand-alone treatment for cure.

\section{Endovascular treatment}

The first report of embolization of an AVM was published by Luessenhop and Spence et al. in 1960 [18] who used methyl methacrylate pellets after a direct carotid puncture. Selective catheterization of the intracranial circulation with microcatheters was first described by Serbinenko et al. [19] and Kerber. Further evolution has occurred with the use of new liquid embolic agents like cyanoacrylate by Drake et al. [20] and Debrun et al. [21]. After years of embolization with $\mathrm{N}$-butyl cyanoacrylate, introduction of a new agent composed of ethylene vinyl alcohol polymer (Onyx, Medtronic, Irvine, CA, USA) has changed the practice of AVMs once more.

Endovascular treatment of cerebral AVMs can be used before open surgery, before radiosurgery, for cure as a stand-alone treatment, to target the weak angioarchitectural points, or for palliative purposes. The advantages of endovascular treatment include minimally invasiveness, immediate angiographic evaluation during and after the treatment, and immediate occlusive effect. Angiography is the gold standard for the diagnosis and treatment planning for AVMs. Angiograms show the location, size, and number of arterial feeders and draining veins and locate weak points such as intranidal aneurysms, flow-related aneurysms, venous drainage stenosis, ectasia, or aneurysm of the draining veins. These angioarchitectural characteristics lead to decision on the treatment strategy for a specific AVM including surgical, endovascular, or radiosurgical techniques.

\subsection{Embolization before surgical resection}

Preoperative embolization reduces the blood loss during surgery and decreases surgery times. By decreasing the size of an AVM, the Spetzler-Martin grade and eventually surgical morbidity-mortality are decreased (Figure 1). Grade 1 and grade 2 AVMs, which are amenable to stand-alone surgery, may benefit from the embolization of the deeply located feeders. Grade 3 AVMs with deep and eloquent location can be treated with embolization preoperatively and surgical morbidity and mortality can be reduced significantly [22]. Presurgical embolization is the most beneficial for grade 3 AVMs.

\subsection{Embolization before radiosurgery}

Obliteration rates after radiosurgery decreases as the size of an arteriovenous malformation increases. Obliteration rate decreases from 80 to $50 \%$ when the size of the AVM increases from 2.5 to $3 \mathrm{~cm}$ [23]. The main aim of embolization before radiosurgery is to decrease the size of the lesion before radiosurgery [24] (Figure 2). AVMs larger than $3 \mathrm{~cm}$ can benefit significantly by embolization. A successful embolization obliterating the periphery of an AVM can help to decrease the required dose for obliteration and negative effects of the radiosurgery on the neighboring tissues [25]. If an AVM has intranidal aneurysms, these aneurysms can be embolized to decrease the bleeding risk during the latency period. Additionally, targeted embolization can be used to obliterate direct arteriovenous shunts in the arteriovenous malformations to increase the efficiency of the radiosurgery [26]. 


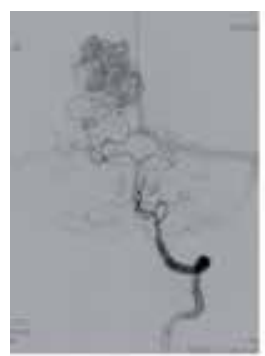

A

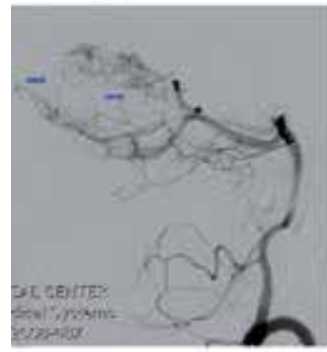

E

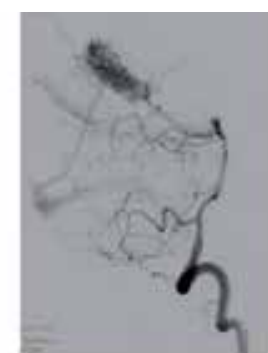

B

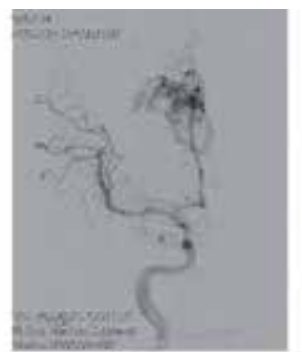

C

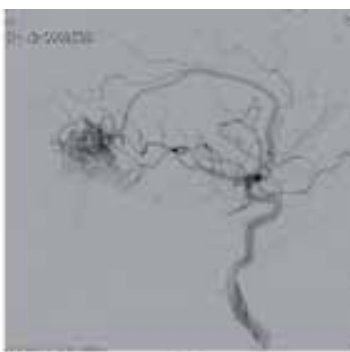

D

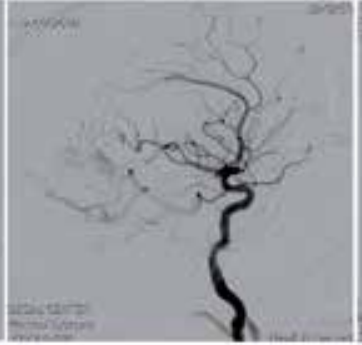

F

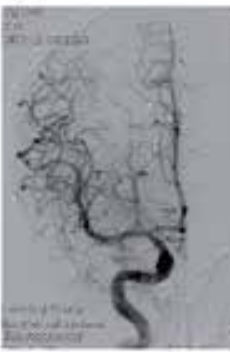

G

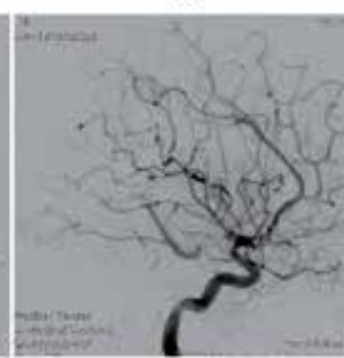

H

Figure 1.

(A-D) A ruptured choroidal AVM that caused intraventricular and subarachnoid hemorrhage in a 26-yearold male. AVM is supplied by the right posterior cerebral artery (PCA), parietooccipital branch, medial and lateral posterior choroidal arteries, and right pericallosal artery from anterior cerebral artery $(A C A)$. ( $E$ and $F$ ) Residual AVM supplied by en passage arterial feeders (arrows) from the posterior choroidal arteries and more than $90 \%$ size reduction after embolization. These en passage feeders were not amenable to embolization and patient went to surgery for AVM resection on the following day after embolization. Control angiogram after surgical resection does not demonstrate any evidence of a residual $A V M(G$ and $H)$.

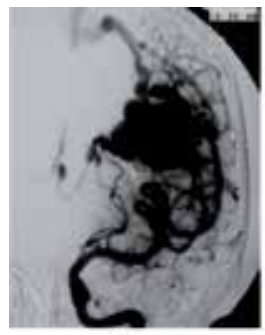

A

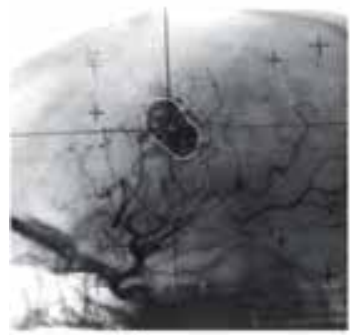

E

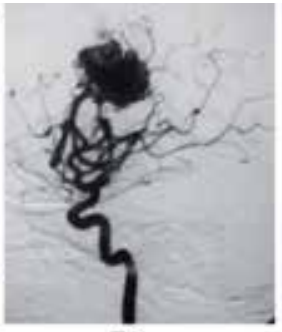

B

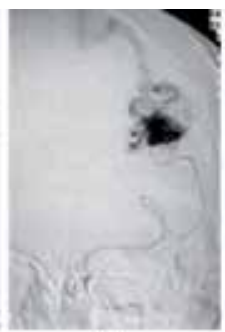

C

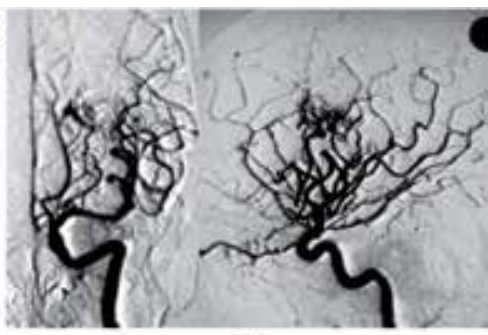

D

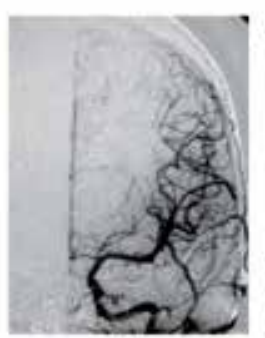

$\mathrm{F}$

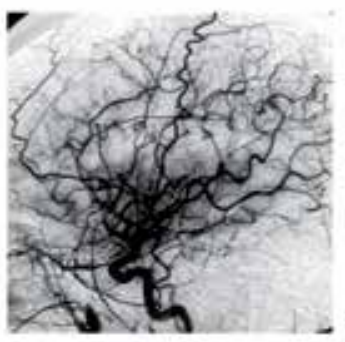

G

Figure 2.

( $A$ and $B$ ) An unruptured left perirolandic AVM supplied by middle cerebral artery branches in a 46-yearold female with headaches. (C) Superselective catheterization and angiogram during preradiosurgical embolization. Postembolization angiograms (D) show at least $90 \%$ size reduction with a small residual lesion in the Rolandic region. Stereotactic surgery is planned (E) following embolization due to eloquent location. Follow-up angiograms 2 years after radiosurgery ( $F$ and $G$ ) demonstrate complete obliteration of the AVM without any residual or recurrent lesion. 
There are conflictive reports in the literature regarding the efficiency of embolization before radiosurgery. Whereas some studies have recently mentioned decreased obliteration rates after embolization [27,28], other studies have demonstrated increased efficacy of radiosurgery with better obliteration rates after embolization [29].

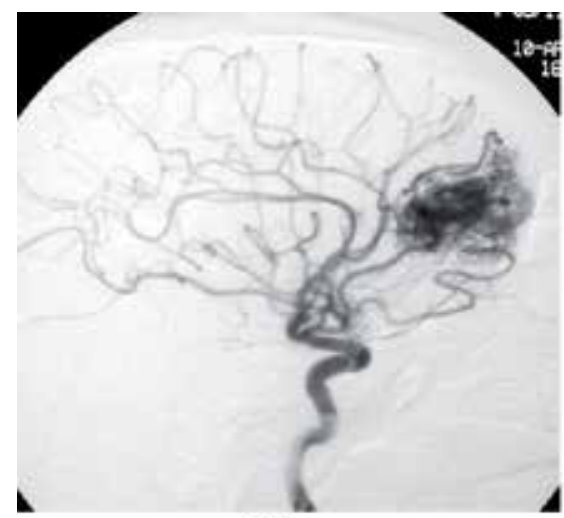

(a)

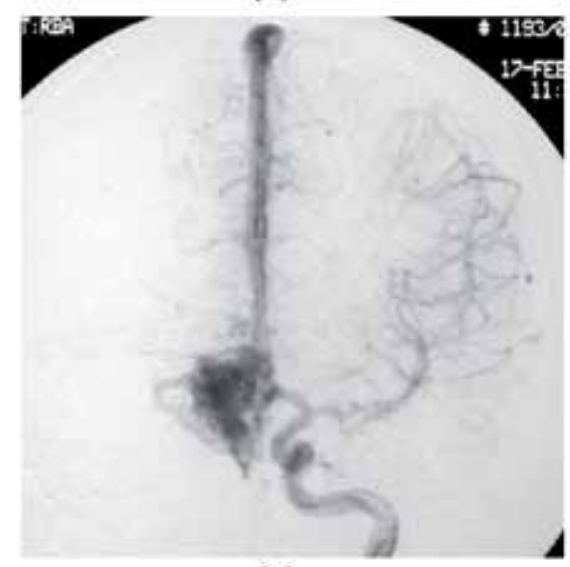

(c)

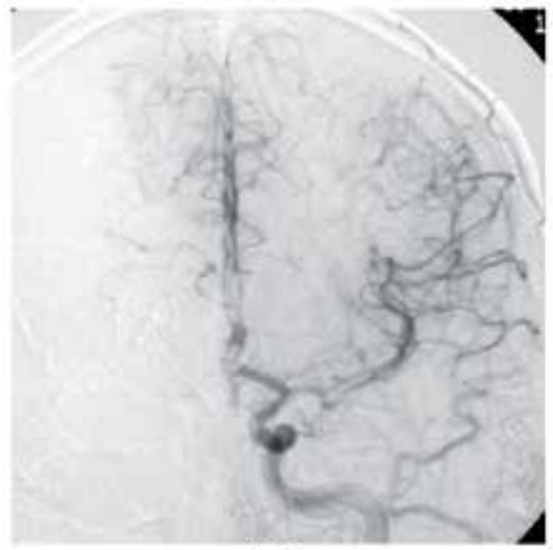

(e)

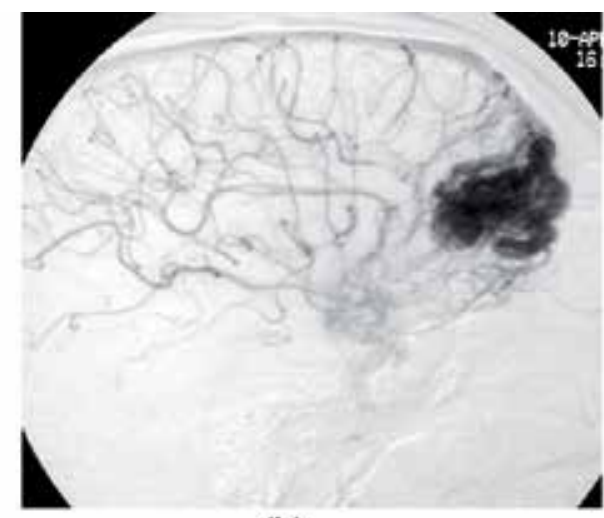

(b)

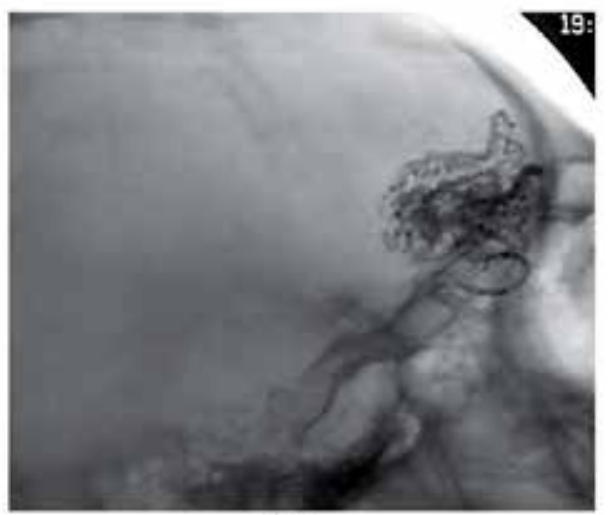

(d)

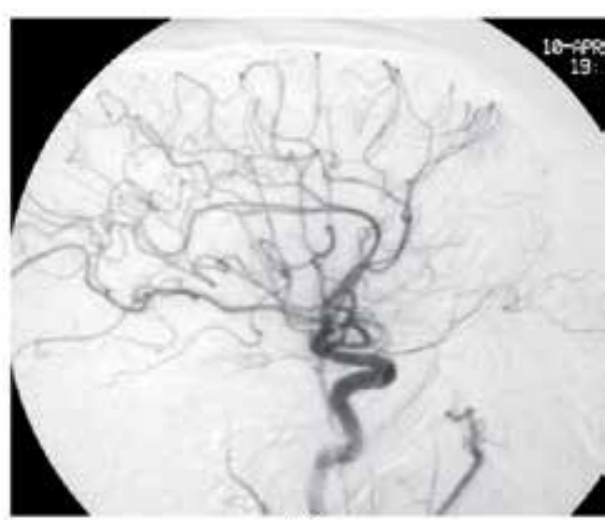

(f)

Figure 3.

(A-C) An unruptured frontopolar AVM supplied by the orbitofrontal and frontopolar branches of the right ACA and drained by a single frontal cortical vein into the superior sagittal sinus. (D) The ETOH cast after embolization of two feeders in the same session with a curative intent. ETOH cast completely matches with the angioarchitecture of the AVM. Final angiograms ( $E$ and $F$ ) show complete obliteration of the AVM without any residual filling. 
In our practice, we recommend embolization before radiosurgery for cases with a volume larger than $10 \mathrm{cc}$, fistulous arteriovenous shunting, and intranidal aneurysms.

\subsection{Curative embolization}

Complete obliteration rates with embolization have been reported between 9.7 and 14\% with NBCA (N-butyl cyanoacrylate) [30]. During the Onyx era, complete obliteration rates with stand-alone embolization rose up to $18-51 \%$ [31]. Even higher cure rates up to $94 \%$ has been reported in smaller series with selective cases [32]. There were higher rates in AVMs with smaller size and a smaller number of arterial feeders (Figure 3). AVMs that have less prominent angiogenic nidus and predominantly fistulous AVMs compared to pure plexiform lesions were also more prone to complete obliteration. Small AVMs with single or less than two feeders with deep and central location are good candidates for stand-alone endovascular treatment. Larger size of the feeding artery also increases the chance of complete angiographic obliteration [33]. According to Valavanis and Yasargil, sulcal AVMs fed by pial arteries are more amenable to safe and effective embolization [34]. Most recent meta-analysis by $\mathrm{Wu}$ et al. [31] found an overall complication rate of

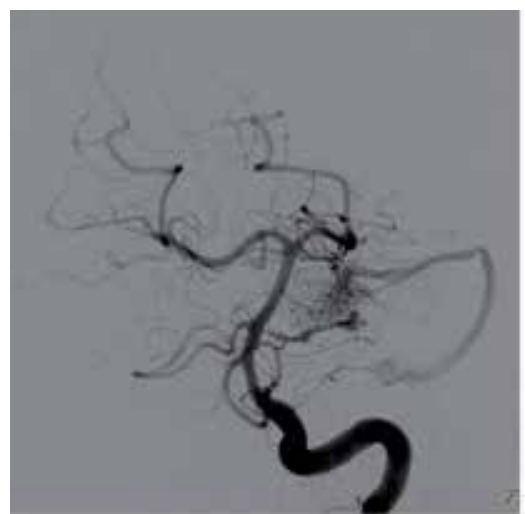

A

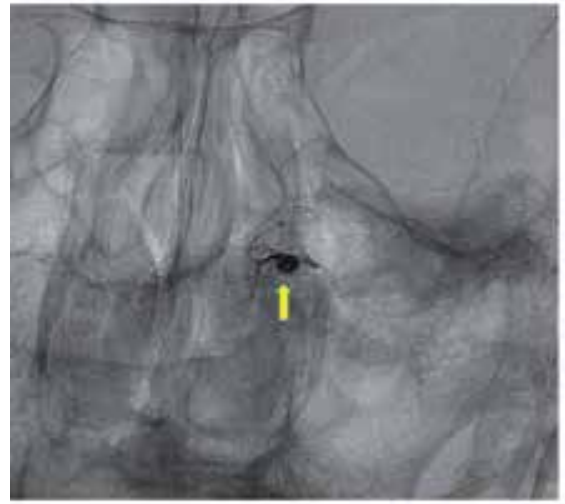

$\mathrm{C}$

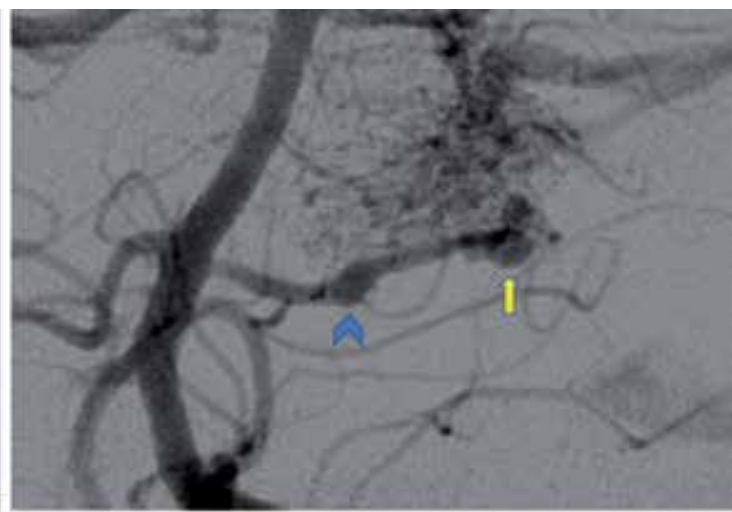

B

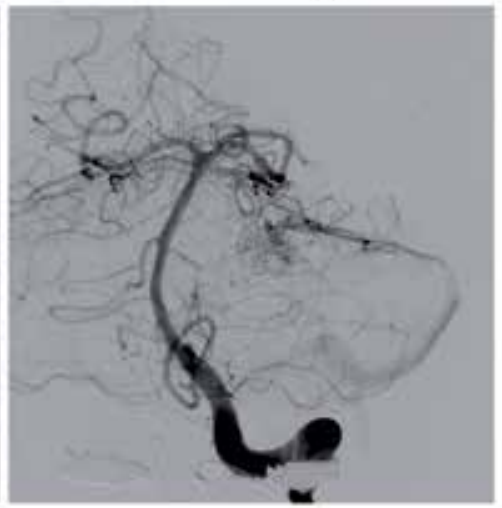

$\mathrm{D}$

Figure 4.

(A) Ruptured cerebellar AVM supplied by the left anterior inferior cerebellar artery (AICA) branches and with drainage into the left transverse sinus. (B) A flow-related aneurysm on the left AICA and multiple intranidal aneurysms. Unsubtracted angiogram (C) after targeted embolization of the left AICA shows NBCA cast within the aneurysm that was most likely the culprit for the previous rupture and hemorrhage. Postembolization angiogram before surgery $(D)$ shows occlusion of multiple aneurysms with residual AVM. 
24.1\% including hemorrhage occurring in $9.7 \%$ of patients and procedure-related mortality rate of $1.5 \%$. These rates are slightly higher compared with studies where the goal was adjunctive embolization before surgery or stereotactic radiotherapy. Even though stand-alone embolization with intent to cure has the potential to be a safe and efficient treatment, it must be preserved for select AVM cases or can be welcomed as an unanticipated result of an adjunctive endovascular treatment.

\subsection{Targeted embolization}

Intranidal aneurysm that may cause recurrent bleeding is the main target for embolization if a complete or near complete obliteration is not feasible (Figure 4). Aneurysms are more frequently seen in AVMs located in the frontal and occipital lobes. Deep arterial feeders not accessible by surgery are also a good target for embolization. Obliteration of the fistulous component of an AVM and decreasing AVM size by embolization may improve the cure rates after radiosurgery.

\subsection{Palliative embolization}

Although there is significant debate on the management of unruptured arteriovenous malformations, intractable seizures or intractable headaches may dictate further treatment rather than medical management. Embolization can decrease the severity of these symptoms by theoretically reducing the steal phenomenon and venous hypertension $[35,36]$. Embolization of the meningeal supply can relieve intractable headaches. Resolution of trigeminal neuralgia after embolization has been reported [37]. One must always consider risk-benefit balance when considering embolization for symptom relief because partial treatment of large AVMs by embolization or surgery may increase the risk of intracranial hemorrhage [27]. On the other hand, Meisel et al. [38] found that partially embolized AVMs have a lower risk of hemorrhage than the risk expected during the natural course of an untreated AVM.

\section{Endovascular technique}

\subsection{Embolization procedure}

Operator needs to know the goal of the endovascular treatment whether it is aiming a complete obliteration, presurgical grade reduction, size reduction before radiosurgery, or a targeted treatment to obliterate weak angioarchitectural points like flow-related aneurysms or intranidal aneurysm causing recurrent hemorrhage. Accordingly, with the specific aims, a game plan can be organized.

In most of the institutions, AVM embolization is performed under general anesthesia. Although some centers advocate conscious sedation over general anesthesia, we do not find it practical to perform embolization in awake patients considering potentially lengthy procedures and absolute need for immobilization during embolysate injection. Triaxial systems with intermediate distal access catheters provide the advantage of much needed stability and momentum in distal and tortuous arterial feeders. A distal access catheter can be navigated to the level of the supraclinoid internal carotid artery, basilar artery, and even in the middle cerebral artery $\mathrm{M} 1$ or posterior cerebral artery $\mathrm{P} 1$ segments to have a better support.

If the aim is cure or significant size reduction, the ideal position of the microcatheter will be as distal as possible and close to the nidus of the AVM. One must be very careful during microcatheter navigation within the arterial feeders that are prone to dissection or perforation. The safest navigation technique is pushing the 
leading microcatheter with a microwire inside and without microwire coming off. Microwire can lead the microcatheter if there is a kink or tortuosity preventing the microcatheter to move forward alone. As soon as the kink or tortuosity is overcome, microwire is withdrawn inside. Navigation technique is different for flowdependent microcatheters like Magic microcatheter (Balt, Montmorency, France). These microcatheters can be pushed with injection of saline and contrast mixtures and gentle pushing. One must pay attention to avoid kinking of these microcatheters because of poor visibility of the microcatheter shaft that tends to fold on itself when there is too much forward momentum. Gentle superselective microcatheter injections demonstrate the component supplied by the pedicle, venous drainage, and speed of shunting. If there is normal parenchymal blush from the same pedicle, another pedicle is chosen to prevent nontargeted embolization. En passage feeders are challenging because it can be difficult to choose them selectively, and distance to the normal main branch is usually very short for a safe injection. Microcatheter is flushed with saline after contrast injection and before introducing the D5 for NBCA or DMSO for $\mathrm{ETOH}$. The working angle with the longest and straightest view of the microcatheter avoiding overlapping of the nidus and microcatheter must be chosen for the superselective angiogram and liquid embolic injection.

Ideal liquid embolic injection aims to penetrate into the nidus, completely obliterate the nidus, and reach to the very proximal portion of the venous outflow (Figure 5). This can be challenging in AVMs with multiple feeders and single draining vein. If the venous outflow is occluded with remaining arterial feeders and a

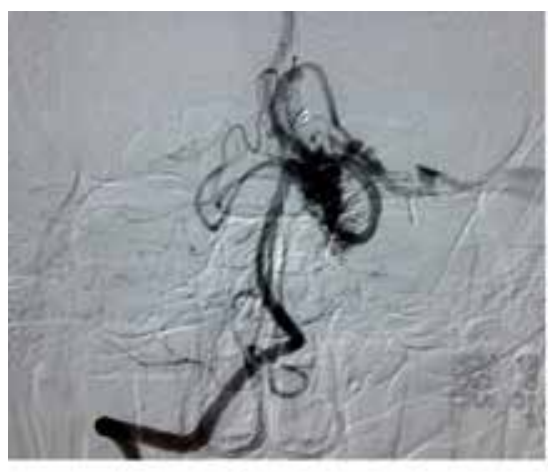

A

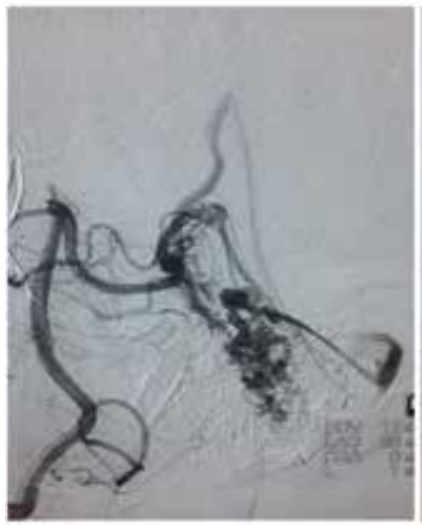

$\mathrm{C}$

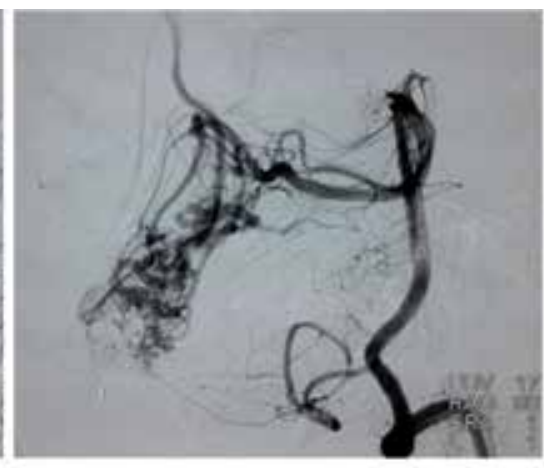

B

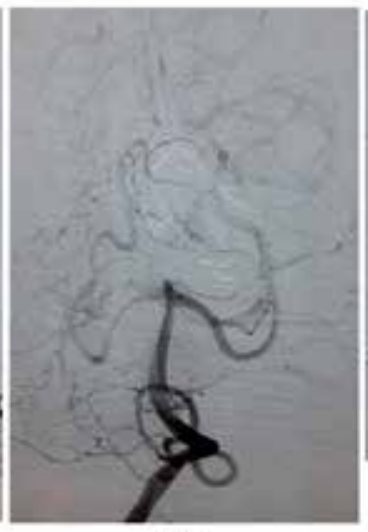

D

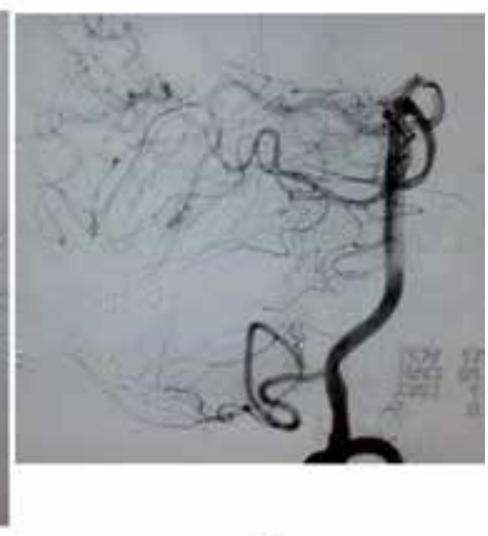

$\mathrm{E}$

Figure 5.

(A-C) A previously ruptured cerebellar AVM in a 57-year-old-male with prior history of surgery for hematoma evacuation and partial resection of the AVM. ( $D$ and $E$ ) Complete obliteration of the AVM after embolization. 


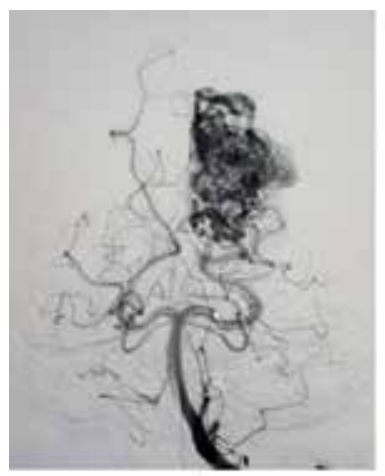

A

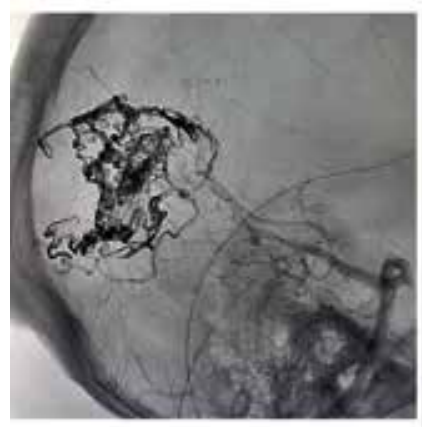

D

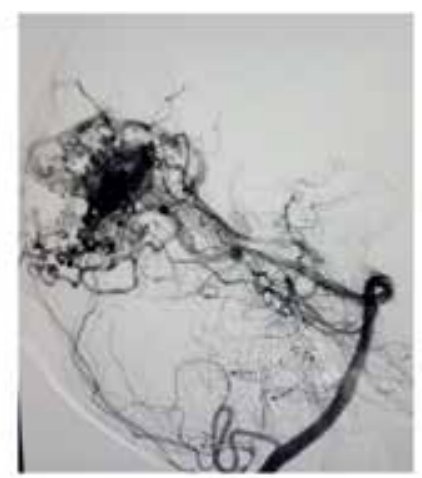

B

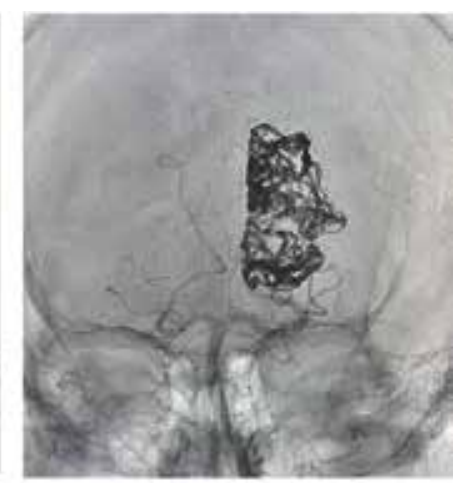

C

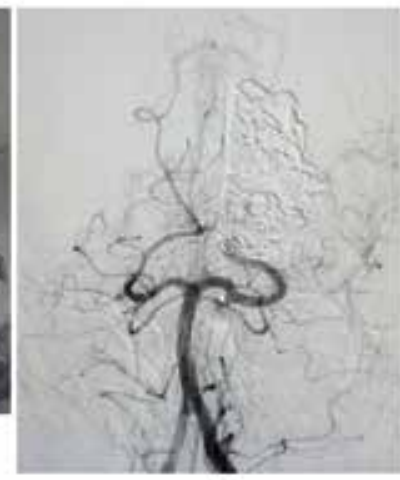

E

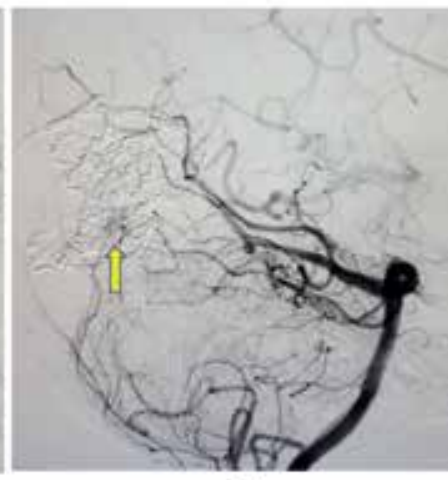

$\mathrm{F}$

Figure 6.

( $A$ and $B$ ) An unruptured posterior fossa AVM. Final angiogram (C and D) after balloon microcatheterassisted embolization shows the onyx cast almost entirely matching with the AVM. (E and F) A tiny residual filling (arrow) without obvious early venous filling. Patient had surgery following embolization for complete resection of the AVM.

large residual nidal component, a normal pressure breakthrough may occur leading to intracranial hemorrhage. Early venous stagnation with a residual persistent AVM can be an ominous sign and may require complete endovascular obliteration of the AVM if possible or immediate surgical resection (Figure 6).

Depending on the angioarchitectural characteristics and preferences and experience of the operator, EVOH or NBCA can be chosen. Although ETOH is used more frequently with the advantages of longer injection times or possibility of halting injections with intermittent angiographic control, NBCA is still preferred by some institutions [39]. Loh and Duckwiler [40] did not find significant differences between two agents in their capacity for at least $50 \%$ volume reduction, complication rates, and intraoperative blood loss. NBCA works through polymerization and requires meticulous preparation in order to prevent contamination with blood or saline that may cause premature polymerization. NBCA is prepared in a separate table or in an isolated part of the main table [40]. Operators change their gloves before preparation to prevent contamination and contact of blood with embolic agents. Microcatheter is flushed with D5 before NBCA injection. NBCA mixture can be tailored depending on the arteriovenous shunting. Most common mixture ratios of NBCA to Ethiodol are 1:3 or 1:2 (NBCA:Ethiodol). Denser mixtures can be used if there is a fistulous component with fast flow into the veins. More diluted mixtures can penetrate better into the nidus with the drawback of higher chance of reflux. Another technique to improve the NBCA penetration into the lesion is flushing the guiding catheter continuously with D5 dextrose solution while injecting the glue [41]. Penetration of the NCBA can be 
stopped by stopping the D5 perfusion. NBCA is an adhesive agent and microcatheter must be removed briskly as soon as the reflux begins. In both NBCA and ETOH injections, microcatheter is removed while applying negative pressure by gentle aspiration.

ETOH (Onyx, Medtronic, Irvine, CA, USA or Squid, Emboflu, Gland,

Switzerland) is a nonadhesive liquid embolic agent, which does not polymerize but precipitates when dissolved with dimethyl sulfoxide (DMSO). ETOH laminates along the venous wall without immediate occlusion of the vessel. Most frequently used ETOH-based liquid embolic agent is Onyx (Medtronic, Irvine, CA, USA). Dead space of the microcatheter is filled with the exact amount of DMSO before ETOH injection. Before injection, we usually mark the screen with a pen showing the landmarks including the most proximal tolerable reflux point on the microcatheter, boundaries of the AVM, and beginning of the venous drainage. Under blank roadmap, ETOH is injected slowly and reflux is watched. In the most popular "plug and push technique," reflux forms a plug of ETOH at the tip of the microcatheter. Some institutions prefer the denser version of ETOH like Onyx 34 (Medtronic, Irvine, CA, USA) to form a plug. After the precipitation of ETOH forming a plug, antegrade penetration into the nidus with new injections is expected. Whenever there is further reflux, penetration into another arterial branch, or early venous penetration, injection is stopped for approximately $90 \mathrm{~s}$. Halting injection for more than 2 min may cause clogging of the microcatheter. If there is no ETOH coming off the microcatheter tip or appearing under blank roadmap 60-90 s after starting injection, injection must be stopped and microcatheter is removed. Clogging of the microcatheter can result in rupture of the microcatheter shaft and extravasation of ETOH [42]. Intermittent angiograms can be done to control the obliteration of the AVM and the status of venous drainage while halting ETOH injections. After a satisfying obliteration is achieved, microcatheter is removed from the system. In ETOH cases, microcatheter is removed by applying constant gentle tension on the microcatheter. If there is a stubborn microcatheter stuck in the ETOH cast, patience with repetitive pulling with constant tension will remove the microcatheter in most of the cases.

Advances in microcatheter technology, development of detachable tip microcatheters, ETOH compatible dual lumen microballoon catheters, and evolution of liquid embolic agents have changed the paradigms in the endovascular treatment.

\subsection{New trends in embolization techniques}

\subsubsection{Balloon-assisted embolization}

DMSO compatible balloon microcatheters with double lumen (Scepter, Microvention Terumo, Aliso Viejo, CA, USA and Eclipse, Balt, Montmorency, France) allow injection of ETOH or NBCA while balloon is inflated within the feeding artery $[43,44]$. This creates a wedge positioning of the microcatheter like situation or forms a transient plug to increase the penetration of liquid embolic with minimal or no reflux. This will theoretically decrease the fluoroscopy time and radiation dose and increase the ease of microcatheter removal after injection. This technique is especially effective in fistulous AVMs with large caliber feeders. Balloon inflation prevents reflux and allows a more controlled injection in flow arrest conditions. Special attention must be paid during inflation in order to prevent rupture of the feeder. If there is a problem with balloon inflation, uninflated balloon should be replaced as it may increase the chance of catheter entrapment [45]. If there is reflux along the inflated balloon, further gentle inflation usually prevents reflux. Extreme caution must be paid during inflation of the balloon within the arterial feeders to prevent overinflation and rupture. 


\subsubsection{Transvenous embolization}

Although this technique recalls the venous outflow occlusion, one of the most fearful situations for a neuroendovascular specialist during AVM embolization, there is increasing use of transvenous embolization in selective cases [45-47]. Deeply located hemorrhagic AVMs with small tortuous feeders or en passage feeders may have complex anatomy precluding the use of transarterial embolization. Transvenous embolization have theoretical advantages of better penetration of the AVM nidus, less risk of ischemic events due to arterial occlusion, and relatively easier navigation through enlarged and usually straighter veins. Significantly high complete obliteration rates between 80 and $100 \%[47,48]$ were reported in the literature. A transjugular venous access with a triaxial system including a sturdy guiding system of an $8 \mathrm{~F}$ sheath and distal access catheter is crucial for safe and efficient practice of transvenous embolization. Alternatively, a $6 \mathrm{~F}$ transjugular access with a $6 \mathrm{~F}$ distal access catheter can be used. Detachable tip microcatheters or balloon microcatheters in feasible anatomy may prevent reflux and occlusion of the venous drainage. Some centers intentionally let an approximately $3 \mathrm{~cm}$ reflux after a longer injection and leave the microcatheter inside the system after cutting the microcatheter shaft at the skin incision site. Mendes et al. [47] did not report any thromboembolism associated with transvenous embolization cases with transjugular access where all microcatheters were left intentionally within the venous system. Arterial access and arterial flow arrest with transient balloon occlusion or simultaneous transarterial embolization should be used if technically feasible. Although there is no established standard practice of transvenous embolization, theoretical criteria to select cases include small lesions with a nidus of $<2 \mathrm{~cm}$, hemorrhagic AVMs, patients who are not good surgical candidates, arterial feeders that are not amenable to transarterial embolization like en passage feeders, lenticulostriate arteries or choroidal arteries, and AVMs preferably with a single drainage vein $[46,48]$. Nonadhesive ETOH should be used for transvenous embolization [49]. Systemic hypotension during and after transvenous embolization is also crucial for decreasing the arterial pressure that must be overcome for better nidal penetration. Another technical challenge for transvenous embolization arises from frequently encountered venous anatomical variations and fragile structure of the cerebral veins that may lead to disastrous consequences in case of vessel injury. One must pay extreme caution for safe transvenous navigation of the microcatheter without leading microwire. Aneurysms, ectasia, or stenosis in the draining vein may preclude transvenous embolization due to high risk of vessel injury during navigation.

\subsubsection{Pressure cooker technique}

Pressure cooker technique (PCT), first described by Chapot et al. [50], applies a plug composed of coils and glue instead of the usual ETOH plug used in the regular plug and push technique. Coils are deployed and NBCA is injected to form a plug. A microcatheter for ETOH injection is placed in the optimal position in the feeding artery followed by a second microcatheter placed in between the tip of the first microcatheter and detachment zone. As described by the sheeping technique, placement of the first microcatheter usually facilitates the navigation of the second security microcatheter [32, 51]. A 1.2F Magic (Balt, Montmorency, France) microcatheter is used in small caliber vessels and Echelon 10 (Medtronic, Irvine, CA, USA) is used as a second microcatheter in relatively larger caliber arterial feeders. Since detachable coils are not compatible with Magic 1.2F microcatheter, injectable flow coils (SPIF, Balt, Montmorency, France) are used. In case of larger caliber 
vessels with high flow arteriovenous shunts, injectable coils may flow unintentionally toward the tip of the first microcatheter. Detachable coils are deployed through a regular 1.7F like Echelon 10 (Medtronic, Irvine, CA, USA) for precise placement of the coil and glue plug. This type of plug is more resistant to ETOH reflux and facilitates a more forceful and continuous ETOH injection. It creates a wedge-like position similar to ETOH injection through the balloon microcatheters. The theoretical advantages compared to balloon-assisted embolization are better navigability of flow-directed microcatheters and avoidance of risk of vessel perforation during balloon inflation.

\section{Complications of the endovascular treatment}

Overall morbidity and mortality rates from the largest series of AVM embolization range from 0 to $22 \%$ and 0 to $3 \%$, respectively [52-56]. Intracranial hemorrhage during or after the embolization can be seen in between 2 and $4.7 \%$. Most common reason for hemorrhage is vessel perforation due to microcatheter or microwire manipulations. Venous outflow obstruction and normal perfusion pressure breakthrough are other mechanisms that may cause bleeding after embolization. Normal perfusion pressure breakthrough is believed to occur due to overdilated capillaries in the parenchyma surrounding the AVM in the setting of steal phenomenon or ischemia. After embolization or resection of the AVM, increased pressure or perfusion in the surrounding parenchyma may cause rupture of the maximally dilated capillaries [57]. Staged embolization is preferred in large AVMs in order to prevent normal perfusion breakthrough phenomenon [58]. If the surgical resection is not planned to immediately follow the embolization, a staged embolization in several sessions is performed. Although there is no established rule, less than $50 \%$ size reduction is aimed in every session in order to prevent postembolization hemorrhage. About 4-6 weeks can be waited in between every session for hemodynamic stabilization. If there is significant slowing of the venous drainage during any moment of embolization, either complete obliteration must be achieved by embolization or surgical resection must be performed urgently following embolization. Therefore, it is utmost important to evaluate the venous drainage pattern before and immediately after the embolization. In rare cases, perforation and bleeding may occur during superselective microcatheter injection in a small branch. If there is any concern for intraoperative rupture or postprocedural neurological deterioration happens, a brain computerized tomography must be obtained to rule out hemorrhage. In case of hematoma with mass effect or impending expansion risk, emergent craniotomy for hematoma evacuation helps minimize neurological deficit and may avoid mortal consequences [59].

Ischemic complications can occur due to thromboembolism from the embolic material or thrombus formation within or along the guiding catheter or microcatheter. Mechanisms or thromboembolism are reflux or nontargeted flow of the liquid embolic into a normal branch during embolization. Heparinization is routinely used during AVM embolizations. Other general complications related to angiographic procedure include groin hematoma, retroperitoneal hematoma, arteriovenous fistula, and contrast nephropathy with similar frequencies compared to other neurointerventional procedures.

Buffalo grading system [60] is used to stratify the risks associated with endovascular treatment with curative intent. Risk of the endovascular treatment increases with number of arterial feeders, smaller diameter of the feeders, and eloquent location. One point is given for AVMs with one or two arterial feeders, 2 points is given 
for three or four arterial feeders, and 3 points is assigned for four or more pedicles. If the diameter of the most feeders is less than $1 \mathrm{~mm}$ or AVM is in an eloquent location, an additional point is given. Starke et al. [61] proposed another scale to predict various factors increasing complication risk in AVM embolizations. This scale is very similar to Spetzler-Martin grading system for surgical morbidity-mortality and includes same factors including size ( 1 point for $<3 \mathrm{~cm}$ and 2 points for $>6 \mathrm{~cm}$ ), eloquent location, and deep venous drainage. They proposed the need for more than one session of embolization as an additional risk factor with increased complication rate. However, Crowley et al. [56] did not find any significant difference in the complication and morbidity/mortality rates depending on the Spetzler-Martin grades. The same study did neither show a significant difference in complication rates between the ETOH versus NBCA cases.

Microcatheter retention may occur between 3 and $8 \%$ of cases [42]. Risk of microcatheter retention increases after longer injections with long reflux, in smaller branches and branches with significant curves and tortuosity. If the vessel accommodating the microcatheter has tortuosity, long refluxes must be avoided. Safe reflux for an efficient push and plug technique is usually between 15 and $20 \mathrm{~mm}$. Smaller vessels with or without tortuosity require a smaller reflux of less than $10 \mathrm{~mm}$ [50]. Although ETOH is nonadhesive and initially microcatheter retention was expected to decrease with ETOH use, clinical practice and experience demonstrated the contrary. The risk increased due to longer injection times and the need for a significant reflux to form a plug for efficient ETOH injection. Loh and Duckwiler [40] found higher incidence of microcatheter retention and difficulty in removing the delivery microcatheter in the first trial comparing Onyx to NBCA, which led to FDA approval of the Onyx. ETOH is less thrombogenic than NBCA and does not cause immediate occlusion in case of an unintended flux.

Development of detachable tip microcatheters including Apollo (Medtronic, Irvine, CA, USA) and Sonic (Balt, Montmorency, France) has substantially decreased the incidences of microcatheter retention [62]. These microcatheters have variable lengths $(1.5$ or $3 \mathrm{~cm})$ of detachable tip segment that allows better estimate of tolerable reflux thanks to markers, and detachment will occur if there is enough reflux to create a tension point. Sonic (Balt, Montmorency, France) microcatheter usually detaches after a long injection with substantial reflux; whereas Apollo (Ev3) microcatheter may not detach in many cases and can be retrieved as a whole without detachment.

Introduction of distal access catheters helped better navigation of very distal tortuous feeders and facilitated retrieval of microcatheters after a long injection. The use of distal access catheters is almost the standard of practice especially for cases with superficial lesions with distal and tortuous feeders. Distal location of a guide catheter eliminates the need for repeated navigation of a microcatheter through a tortuous supraclinoid internal carotid, anterior, middle cerebral artery, or basilar artery.

Although recanalization after complete obliteration of an AVM is extremely rare, there is still risk of recanalization that mandates angiographic follow-up after complete obliteration. Potts et al. [63] in their extensive review of multiple series of AVMs cured with embolization found $4.5 \%$ recurrence rate on follow-up angiography. However, there was not any case of recurrence with rupture or any adverse event during follow-up period. Mechanisms that may lead to recurrence include incomplete embolization due to nonvisualization of a component during initial embolization, mass effect from a hematoma, or recanalization of an initially thrombosed compartment. 


\section{Author details}

Cagin Senturk ${ }^{1,2}$

1 University of California Irvine Medical Center, United States

2 St. Jude Medical Center, Fullerton, California, United States

*Address all correspondence to: caginsenturk@yahoo.com

\section{IntechOpen}

(C) 2019 The Author(s). Licensee IntechOpen. This chapter is distributed under the terms of the Creative Commons Attribution License (http://creativecommons.org/licenses/ by/3.0), which permits unrestricted use, distribution, and reproduction in any medium, provided the original work is properly cited. (cc) BY 


\section{References}

[1] Yasargil MG. AVM of the Brain, History, Embryology, Pathological Considerations, Hemodynamics, Diagnostic Studies, Microsurgical Anatomy. Stuttgart, Germany: George Thieme Verlag; 1987

[2] Jessurun GA, Kamphuis DJ, van der Zande FH, Nossent JC. Cerebral arteriovenous malformations in The Netherlands Antilles. High prevalence of hereditary hemorrhagic telangiectasiarelated single and multiple cerebral arteriovenous malformations. Clinical Neurology and Neurosurgery. 1993;95(3):193-198

[3] Brown RD Jr, Wiebers DO, Torner JC, O'Fallon WM. Incidence and prevalence of intracranial vascular malformations in Olmsted County, Minnesota, 1965 to 1992. Neurology. 1996;46:949-952

[4] Stapf C, Mast H, Sciacca RR, et al. The New York Islands AVM study design, study progress, and initial results. Stroke. 2003;34:e29-e33

[5] Kikuchi K, Kowada M, Sasajima H. Vascular malformations of the brain in hereditary hemorrhagic telangiectasia (Rendu-Osler-Weber disease). Surgical Neurology. 1994;41:374-380

[6] Laufer L, Cohen A. Sturge-Weber syndrome associated with a large left hemispheric arteriovenous malformation. Pediatric Radiology. 1994;24:272-273

[7] da Costa L, Wallace MC, Ter Brugge KG, O'Kelly C, Willinsky RA, Tymianski $M$. The natural history and predictive features of hemorrhage from brain arteriovenous malformations. Stroke. 2009;40:100-105

[8] Stapf C, Mast H, Sciacca RR, Choi JH, Khaw AV, Connolly ES, et al. Predictors of hemorrhage in patients with untreated brain arteriovenous malformation. Neurology. 2006;66(9):1350-1355
[9] Hurst R, Rosenwasser R.

Interventional neuroradiology. New

York, USA: Informa Healthcare; 2008

[10] Hernesniemi JA, Dashti R, Juvela S, Väärt K, Niemelä M, Laakso A. Natural history of brain arteriovenous malformations: A long-term follow-up study of risk of hemorrhage in 238 patients. Neurosurgery. 2008;63(5):823-829

[11] Mast H, Young WL, Koennecke HC, Osipov A, Pile-Spellman J,

Hacein-Bey L, et al. Risk of spontaneous haemorrhage after diagnosis of cerebral arteriovenous malformation. Lancet.

1997;350:1065-1068

[12] Spetzler RF, Hargraves RW, MccormickPW, ZabramskijM, Flom RA, Zimmermans RS. Relationship of perfusion pressure and size to risk of hemorrhage from arteriovenous malformations. Journal of Neurosurgery. 1992;76:918-923

[13] Ondra SL, Troupp H, George ED, Schwab K. The natural history of symptomatic arteriovenous malformations of the brain: A 24-year follow-up assessment. Journal of Neurosurgery. 1990;73:387-391

[14] Mohr JP, Parides MK, Stapf C, Moquete E, Moy CS, Overbey JR, et al. International ARUBA investigators. Medical management with or without interventional therapy for unruptured brain arteriovenous malformations (ARUBA): A multicentre, nonblinded, randomised trial. Lancet. 2014;383:614-621

[15] Derdeyn CP, Zipfel GJ, Albuquerque FC, Cooke DL, Feldmann E, Sheehan JP, et al. American Heart Association Stroke Council. Management of brain arteriovenous malformations: A scientific statement for healthcare professionals from 
the American Heart Association/ American Stroke Association. Stroke. 2017;48(8):e200-e224. DOI: 10.1161/ STR.0000000000000134

[16] Spetzler RF, Martin NA. A proposed grading system for arteriovenous malformations. Journal of Neurosurgery. 1986;65:476-483

[17] Spetzler RF, Ponce FA. A 3-tier classification of cerebral arteriovenous malformations. Journal of Neurosurgery. 2011;114:842-849

[18] Luessenhop AJ, Spence WT. Artificial embolization of cerebral arteries. Report of use in a case of arteriovenous malformation. Journal of the American Medical Association. 1960;172:1153-1155

[19] Serbinenko FA. Six hundred endovascular neurosurgical procedures in vascular pathology. A ten-year experience. Acta Neurochirurgica. Supplementum (Wien). 1979;28:310-311

[20] Drake CG. Cerebral arteriovenous malformations: Considerations for and experience with surgical treatment in 166 cases. Clinical Neurosurgery. 1979;26:145-208

[21] Debrun G, Vinuela F, Fox A, Drake CG. Embolization of cerebral arteriovenous malformations with bucrylate. Journal of Neurosurgery. 1982;56:615-627

[22] Lawton MT. Spetzler-Martin grade III arteriovenous malformations: Surgical results and a modification of the grading scale. Neurosurgery. 2003;52(4):740-749

[23] Pollock BE, Gorman DA, Schomberg PJ, Kline RW. The Mayo Clinic gamma knife experience: Indications and initial results. Mayo Clinic Proceedings. 1999;74(1):5-13
[24] Henkes H, Nahser HC, Berg-Dammer E, Weber W, Lange S, Kühne D. Endovascular therapy of brain AVMs prior to radiosurgery. Neurological Research. 1998;20(6):479-492

[25] Yuki I, Kim RH, Duckwiler G, Jahan R, Tateshima S, Gonzalez N, et al. Treatment of brain arteriovenous malformations with high-flow arteriovenous fistulas: Risk and complications associated with endovascular embolization in multimodality treatment. Journal of Neurosurgery. 2010;113(4):715-722. DOI: 10.3171/2009.9.JNS081588

[26] Blackburn SL, Ashley WW Jr, Rich KM, Simpson JR, Drzymala RE, Ray WZ, et al. Combined endovascular embolization and stereotactic radiosurgery in the treatment of large arteriovenous malformations. Journal of Neurosurgery. 2011;114(6):1758-1767

[27] Andrade-Souza YM, Ramani M, Scora D, Tsao MN, terBrugge K, Schwartz ML. Embolization before radiosurgery reduces the obliteration rate of arteriovenous malformations. Neurosurgery. 2007;60(3):443-451

[28] Schwyzer L, Yen CP, Evans A, Zavoian S, Steiner L. Long-term results of gamma knife surgery for partially embolized arteriovenous malformations. Neurosurgery. 2012;71(6):1139-1147

[29] Pierot L, Kadziolka K, Lanoix O, Rousseaux P. Combined treatment of brain arteriovenous malformations using Onyx embolization followed by radiosurgery. AJNR. American Journal of Neuroradiology. 2013 Jul;34(7):1395400. DOI: 10.3174/ajnr.A3409

[30] Elsenousi A, Aletich VA, Alaraj A. Neurological outcomes and cure rates of embolization of brain 
arteriovenous malformations with n-butyl cyanoacrylate or Onyx: A metaanalysis. Journal of Neurointerventional Surgery. 2016;8(3):265-272. DOI: 10.1136/neurintsurg-2014-011427

[31] Wu EM, El Ahmadieh TY, McDougall CM, Aoun SG, Mehta N, Neeley OJ, et al. Embolization of brain arteriovenous malformations with intent to cure: A systematic review. Journal of Neurosurgery. 2019;1:1-12. DOI: $10.3171 / 2018.10$. JNS181791

[32] Abud DG, Riva R, Nakiri GS, Padovani F, Khawaldeh M, Mounayer C. Treatment of brain arteriovenous malformations by double arterial catheterization with simultaneous injection of onyx: Retrospective series of 17 patients. AJNR. American Journal of Neuroradiology. 2011;32:152-158

[33] Strauss I, Frolov V, Buchbut D, Gonen L, Maimon S. Critical appraisal of endovascular treatment of brain arteriovenous malformation using onyx in a series of 92 consecutive patients. Acta Neurochirurgica. 2013;155:611-617

[34] Valavanis A, Yaşargil MG. The endovascular treatment of brain arteriovenous malformations. Advances and Technical Standards in Neurosurgery. 1998;24:131-214

[35] Mast H, Mohr JP, Osipov A, Pile-Spellman J, Marshall RS, Lazar RM, et al. 'Steal' is an unestablished mechanism for the clinical presentation of cerebral arteriovenous malformations. Stroke. 1995;26:1215-1220

[36] Rosenkranz M, Regelsberger J, Zeumer H, Grzyska U. Management of cerebral arteriovenous malformations associated with symptomatic congestive intracranial hypertension. European Neurology. 2008;59:62-66
[37] Simon SD, Yao TL, Rosenbaum BP, Reig A, Mericle RA. Resolution of trigeminal neuralgia after palliative embolization of a cerebellopontine angle arteriovenous malformation. Central European Neurosurgery. 2009;70:161-163

[38] Meisel HJ, Mansmann U, Alvarez H, Rodesch G, Brock M, Lasjaunias P. Effect of partial targeted N-butyl-cyanoacrylate embolization in brain AVM. Acta Neurochirurgica. 2002;144:879-887

[39] Howington JU, Kerber CW, Hopkins LN. Liquid embolic agents in the treatment of intracranial arteriovenous malformations. Neurosurgery Clinics of North America. 2005;16:355-363

[40] Loh Y, Duckwiler GR. A prospective, multicenter, randomized trial of the onyx liquid embolic system and N-butyl cyanoacrylate embolization of cerebral arteriovenous malformations. Journal of Neurosurgery. 2010;113:733-741

[41] Gailloud P. Endovascular treatment of cerebral arteriovenous malformations. Techniques in Vascular and Interventional Radiology. 2005;8(3):118-128

[42] Senturk C. Mechanical removal of migrated Onyx due to microcatheter rupture during AVM embolization: A technical case report. Cardiovascular and Interventional Radiology.

2015;38(6):1654-1657

[43] Jagadeesan BD, Grigoryan M, Hassan AE, Grande AW, Tummala RP. Endovascular balloonassisted embolization of intracranial and cervical arteriovenous malformations using dual-lumen coaxial balloon microcatheters and Onyx: Initial experience. Neurosurgery;73(2 Suppl Operative):238-243 
[44] Paramasivam S, Niimi Y, Fifi J, Berenstein A. Onyx embolization using dual-lumen balloon catheter: Initial experience and technical note. Journal of Neuroradiology. 2013;40(4):294-302. DOI: 10.1016/j.neurad.2013.08.001

[45] Kessler I, Riva R, Ruggiero M, Manisor M, Al-Khawaldeh M, Mounayer C. Successful transvenous embolization of brain arteriovenous malformations using Onyx in five consecutive patients. Neurosurgery. 2011;69(1):184-193

[46] Choudhri O, Ivan ME, Lawton MT. Transvenous approach to intracranial arteriovenous malformations: Challenging the axioms of arteriovenous malformation therapy? Neurosurgery. 2015;77(4):644-651

[47] Mendes GA, Iosif C, Silveira EP, Waihrich E, Saleme S, Mounayer C. Transvenous embolization in pediatric plexiform arteriovenous malformations. Neurosurgery. 2016;78(3):458-465. DOI: 10.1227/NEU.0000000000001057

[48] Consoli A, Renieri L, Nappini S, Limbucci N, Mangiafico S. Endovascular treatment of deep hemorrhagic brain arteriovenous malformations with transvenous onyx embolization. AJNR. American Journal of Neuroradiology. 2013;34(9):1805-1811

[49] Chen CJ, Norat P, Ding D, Mendes GAC, Tvrdik P, Park MS, et al. Transvenous embolization of brain arteriovenous malformations: A review of techniques, indications, and outcomes. Neurosurgical Focus. 2018;45(1):E13. DOI: 10.3171/2018.3.FOCUS18113

[50] Chapot R, Stracke P, Velasco A, Nordmeyer H, Heddier M, Stauder M, et al. The pressure cooker technique for the treatment of brain AVMs. Journal of Neuroradiology. 2014;41(1):87-91. DOI: 10.1016/j.neurad.2013.10.001
[51] Chapot R, Nordmeyer H, Heddier M, Velasco A, Schooss P, Stauder $\mathrm{M}$, et al. The sheeping technique or how to avoid exchange maneuvers. Neuroradiology. 2013;55(8):989-992

[52] Deruty R, Pelissou-Guyotat I, Amat D, Mottolese C, Bascoulergue Y, Turjman F, et al. Multidisciplinary treatment of cerebral arteriovenous malformations. Neurological Research. 1995;17(3):169-177

[53] Weber W, Kis B, Siekmann R, Kuehne D. Endovascular treatment of intracranial arteriovenous malformations with onyx: Technical aspects. AJNR. American Journal of Neuroradiology. 2007;28:371-377

[54] Van Rooij WJ, Sluzewski M, Beute GN. Brain AVM embolization with Onyx. AJNR. American Journal of Neuroradiology. 2007;28:172-177

[55] Saatci I, Geyik S, Yavuz K, Cekirge HS. Endovascular treatment of brain arteriovenous malformations with prolonged intranidal Onyx injection technique: Long-term results in 350 consecutive patients with completed endovascular treatment course. Journal of Neurosurgery. 2011;115:78-88

[56] Crowley RW, Ducruet AF, Kalani MY, Kim LJ, Albuquerque FC, McDougall CG. Neurological morbidity and mortality associated with the endovascular treatment of cerebral arteriovenous malformations before and during the Onyx era. Journal of Neurosurgery. 2015;122:1492-1497

[57] Sekhon LH, Morgan MK, Spence I. Normal perfusion pressure breakthrough: The role of capillaries. Journal of Neurosurgery. 1997;86:519-524

[58] Andrews BT, Wilson CB. Staged treatment of arteriovenous malformations of the brain. Neurosurgery. 1987;21:314-323 
[59] Iwama T, Yoshimura K, Keller E, ImhofHG, KhanN, Leblebicioglu-KönuD, et al. Emergency craniotomy for intraparenchymal massive hematoma after embolization of supratentorial arteriovenous malformations. Neurosurgery. 2003;53(6):1251-1258 discussion 1258-1260

[60] Dumont TM, Kan P, Snyder KV. A proposed grading system for endovascular treatment of cerebral arteriovenous malformations: Buffalo score. Surgical Neurology International. 2015;6:3. DOI: 10.4103/2152-7806.148847

[61] Starke RM, Komotar RJ, Otten ML, Hahn DK, Fischer LE, Hwang BY, et al. Adjuvant embolization with N-butyl cyanoacrylate in the treatment of cerebral arteriovenous malformations: Outcomes, complications, and predictors of neurologic deficits. Stroke. 2009;40:2783-2790

[62] Altschul D, Paramasivam S, Ortega-Gutierrez S, Fifi JT, Berenstein A. Safety and efficacy using a detachable tip microcatheter in the embolization of pediatric arteriovenous malformations. Child's Nervous System. 2014;30(6):1099-1107. DOI: 10.1007/ s00381-014-2404-9

[63] Potts MB, Zumofen DW, Raz E, Nelson PK, Riina HA. Curing arteriovenous malformations using embolization. Neurosurgical Focus. 2014;37(3):E19. DOI: 10.3171/2014.6.FOCUS1422 



\title{
Advocating Intraluminal Radiation Therapy in Cerebral Arteriovenous Malformation Treatment
}

\author{
Nitzan Hirsh, Amir Arthur and Saar Golan
}

\begin{abstract}
In 2014, ARUBA (a randomized trial on cerebral Arteriovenous Malformation AVM) found patients treated using prevalent interventional strategies are three times more likely to suffer a stroke/die compared with those treated conservatively (blood pressure reduction). Subsequent controversy led the European societies dealing with AVM to organize a consensus conference. Among the statements made was: “There may be indications for treating patients with higher Spetzler-Martin (SM) grades, based on a case-to-case consensus decision of the experienced team". Thus, a clear accord emerges. There is a lacuna/weakness of interventional modalities when addressing high SM grade AVMs. This lack of a clear treatment choice originated our review. We attempt to identify the advantages and challenges of each present treatment/evaluation modality and highlight core requirements for future strategies. We conclude that existing modalities provide substantial recent improvements, yet the core challenge persists. Finally, we advocate testing a novel modality - intraluminal radiotherapy (active implants) by exploiting the "candy wrapper" or edge effect. If proven effective, this approach could offer gradual vessel occlusion with minimal abrupt hemodynamic changes known to induce hemorrhage, the lowest recurring session number (reduced costs), minimally invasive attributes and very low radiation (dose/dose rate) kinetics minimizing potential Adverse Radiation Effects (AREs).
\end{abstract}

Keywords: arteriovenous malformation, hemorrhage, embolization, radiosurgery, gamma knife, surgical resection, intraluminal radiation therapy

\section{Introduction}

AVM is a tangled web of blood vessels, in which arteries directly transition into veins without intermediate microcirculation elements that provide perfusion to surrounding tissue. The AVM blood vessels are called a nidus and have little resistance to flow compared with a normal capillary bed. Such structure manifests violent flows which result in increased hemorrhage risks. Due to growing use of advanced imaging modalities, there has been increased incidental detection of cerebral AVMs. When detected, there is impending need for treatment since stroke 
chance for an unruptured and untreated AVM is $~ 20-40 \%$ per decade [1]. About $38-71 \%$ of patients presenting brain AVM suffer intracranial hemorrhage [2]. Given these statistics, interventional treatment appears vital. Currently, three prevalent modalities exist: endovascular embolization, stereotactic radiosurgery (SRS), and surgical resection. Each modality conveys a wide risk array (safety/efficacy). Thus, many AVMs ultimately remain untreated (see further data below). Unfortunately, these are typically the larger AVMs that could better benefit from intervention ( $76 \%$ of AVMs having a nidus $<30 \mathrm{~mm}$ are treated; compared with $\sim 57 \%$ of those having a nidus of $30-59 \mathrm{~mm}$ and only $\sim 14 \%$ of those having a nidus $>60 \mathrm{~mm}$ ) [3]. Moreover, embolization typically necessitates a series of interventional procedures/ sessions (up to 11, on average 2.6) [4]. Each procedure involves patient hospitalization, advanced imaging, general anesthesia, and a high-risk operation but, most importantly, exhibits a 3.2\% chance of significant complications [4]. Finally, due to a low obliteration likelihood (11-40\%), embolization is not recommended as a single-modality therapy and is usually combined with radiosurgery. Radiosurgery (if successful) takes 1-3 years to achieve obliteration. Thus, patients remain at hemorrhage risk for a lengthy treatment period [5]. To conclude, many patients with cerebral AVM benefit less from current prevalent treatment modalities that carry high risks, costs, and intensive procedures and, even if eventually effective, take years to complete. In 2014, the largest randomized trial on AVMs (ARUBA trial) found that patients treated using the prevalent interventional strategies were three times more likely to suffer a stroke or die compared with those treated only for blood pressure reduction [6]. ARUBA elicited a plethora of reactions. Some were relatively supportive, but many more criticized the study methods and outcomes. Eventually, the controversy led the European societies dealing with AVM to organize a consensus conference. A clear accord emerges. There is, indeed, a lacuna or at least weakness of interventional modalities when addressing high SM grade AVMs. The lack of clear treatment choice for a pathology with a point prevalence of $\sim 18 / 100,000$ in adults responsible for $4 \%$ of all primary intracerebral hemorrhages is the motivation for this review [7].

\subsection{Definitions and angioarchitecture}

AVM features a vascular region lacking transition hierarchy, where arteries change directly into veins (Figure 1) [13]. AVM physical appearance is a welldefined enclosed volume of entangled blood vessels mostly known as a "nidus." Arteries entering the nidus are termed "feeding arteries" and veins leaving it are termed "draining veins" (Figure 2) [9].

AVMs may appear in virtually every vascular body region. Clinically, significant AVMs are mainly classified as CNS (cerebral), pulmonary, abdominal, renal,
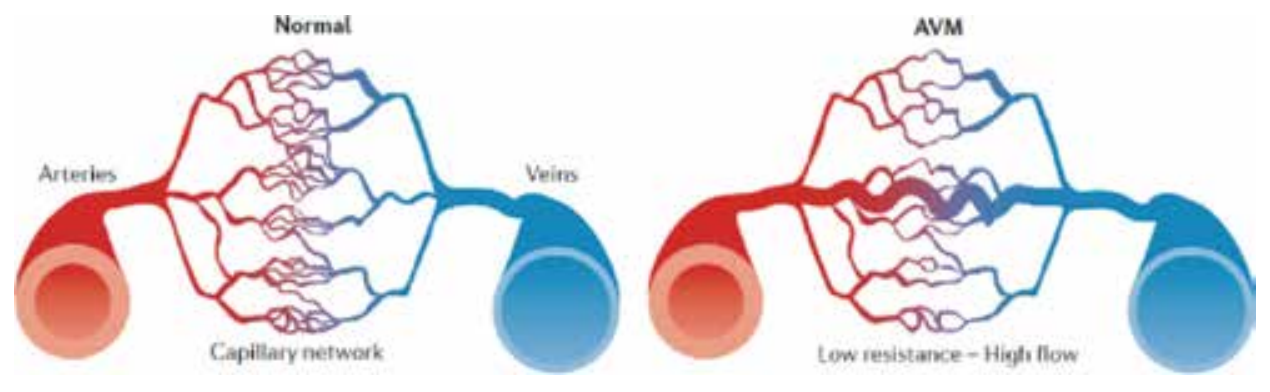

Figure 1.

AVM angioarchitecture [9]. 
hepatic, and peripheral [8-16]. This chapter focuses on cerebral AVMs due to the high risks and challenging treatment involved with this class. Nidus sizes vary in diameter between 1 and $10 \mathrm{~cm}$. AVMs $<3 \mathrm{~cm}$ are classified as "small." AVMs between 3 and $6 \mathrm{~cm}$ are classified as "medium." AVMs $>6 \mathrm{~cm}$ are considered as "large" [8]. The majority of AVMs are medium sized ( 55\%), followed by small $(\sim 38 \%)$ and then large $(\sim 7 \%)$ [17, 18]. The nidus structure does not typically obstruct blood flow; in fact, the opposite is true (see below). Thus, the organ function is usually preserved. However, in rare cases (especially large AVMs), the nidus is orientated or structured in a way that impedes perfusion (steal phenomena) [9]. Here, clinical features of ischemia or lack of brain function may be present [17].

\subsection{Pathophysiology}

AVMs are considered as a major cause of intracerebral hemorrhage, particularly in the young population (33.5\% for age<20years) [19]. From a fluid mechanics perspective, arterioles constitute the main flow resistance/drop of the vascular tree (Figure 3, OpenStax CNX) via a mechanism of rapid increase of surface contact between the blood and the endothelial surface and thus shear stress induction. Lack of arterioles causes minor pressure drops across the nidus. The mean arterial blood pressure difference between normal and AVM cases is $\sim 40 \mathrm{mmHg}$
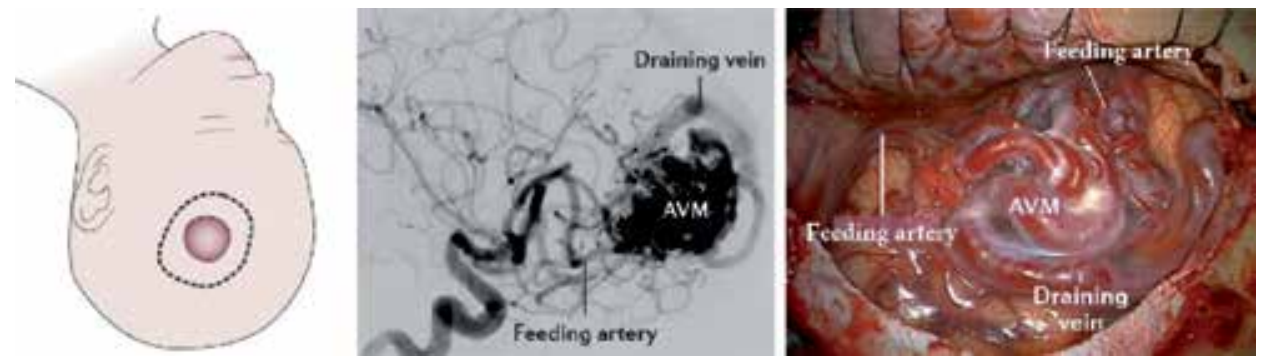

Figure 2.

AVM nidus. Physical location (left), angiogram (center), and pre-surgical anatomy (right) [9].

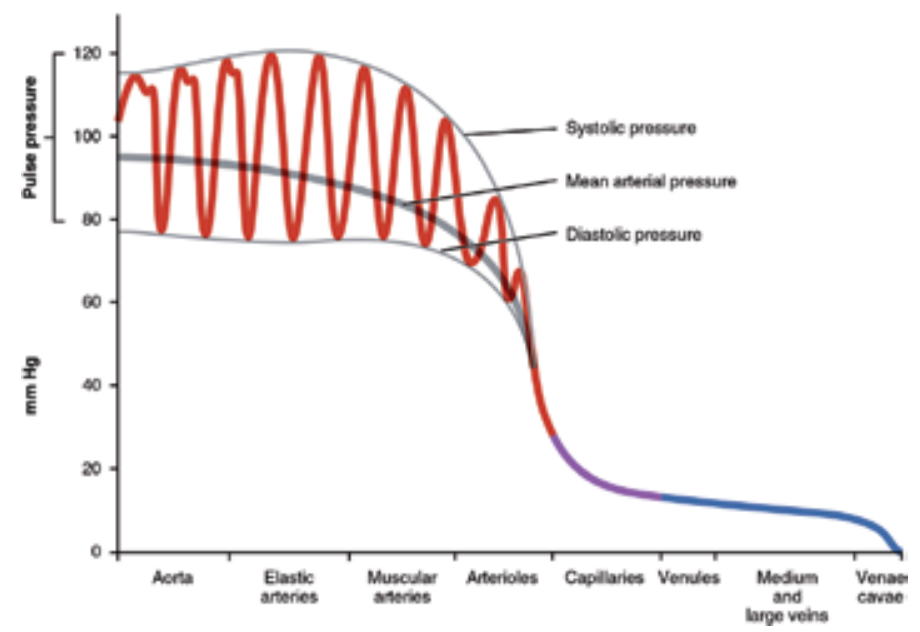

Figure 3.

Pressure drop along the vascular tree. 
and has become an indicator for potential hemorrhages [20]. These findings are confirmed by modern 4D flow magnetic resonance imaging (MRI) and highresolution magnetic resonance angiography (HR 3D MRA) [21, 22]. The CNS vascular network is not equipped to endure pressure drops exceeding $10 \mathrm{mmHg}$, particularly not the structurally thin veins. Thus, the biomechanical aspects of AVM pressures, flows, and shear stresses play a major part in the structural failure of its vessels' vascular walls.

\subsection{Pathogenesis}

AVM is generally considered congenital and/or of developmental origin. Beneš and Bradác describe several genetic pathways associated with AVM pathogenesis, such as hereditary hemorrhagic telangiectasia (HHT, an autosomal dominant genetic disease) [23]. Typically, the genes Endoglin, ALK-1 (ACVRL1, TGF- $\beta$ related), and SMAD4 are implicated [24]. These genes also participate in angiogenesis and vascular remodeling. The main signaling pathways disrupted are: (i) TGF- $\beta$-cellular growth, communication, and inflammatory processes; (ii) NOTCH—angiogenesis, considered critical for arterial fate determination; (iii) MAPK(1/3) — physiological and pathological cell proliferation; and (iv) PI3K/Akt—cell cycle regulation.

\subsection{Epidemiology, natural history, and clinical features}

Fast-flow lesions' prevalence is estimated to be 1-2 out of 10,000 people or $1.2-1.3$ in 100,000 per person-year [23]. AVMs are found in $0.05 \%$ of all brain MRIs [25]. In the USA and Canada, 5000 new cases are detected yearly [26]. Demographic, morphological, and clinical (particularly age-related) characteristics do vary, but many important pathology-related statistics (e.g., rate of incident hemorrhages) remain roughly similar worldwide [17]. In South Africa, male gender and African-black origin were found to be strong indicators for AVM seizure development [27]. Frontal (20.4\%), parietal (22.2\%), temporal $(16.6 \%)$, and cerebellar $(14.0 \%)$ are the most common anatomical brain regions to present AVMs [17, 27]. Supratentorial lesions account for $90 \%$ of brain AVMs [28]. Intracranial hemorrhage is the most common clinical presentation (30-82\% of lesions) [29]. AVMs account for $1-2 \%$ of all strokes and $2-4 \%$ of all non-traumatic intracerebral hemorrhages [23]. However, the unique age distribution among stroke etiologies (the median age for an AVM patient is 32 years old [30]) suggests that AVMs are responsible for more than a third of hemorrhagic strokes in young adults [19]. Furthermore, some studies suggest that higher hemorrhage risk values of $4.6 \%$ [31] and $30 \%$ of lesions are subarachnoid [32]. These are considered highly catastrophic and present the mortality rates of $25-50 \%$ [33]. Main factors influencing hemorrhage risk statistics are initial hemorrhagic $(7.48 \%)$ or seizure $(4.16 \%)$ presentations, associated aneurysms $(6.93 \%)$, and deep venous drainage $(5.42 \%)$ [31]. Other clinical representations are neurological deficits (24\%), chronic headaches (19\%), and focal or generalized seizures (46\%) [17]. The American Association of Neurological Surgeons (AANS) provides a wide symptoms list-seizures, muscle weakness or paralysis, loss of coordination, difficulties carrying out organizational tasks, dizziness, headaches, visual disturbances, language problems, abnormal sensations, memory deficits, mental confusion, hallucinations, and dementia [34]. Annual mortality rates vary between 0.7 and 2.9\% [23]. Considering an estimated number of 300,000 US patients, this results in $2100-8700$ annual mortalities in the USA alone. 


\subsection{Brief history}

Early AVM descriptions may date back to 1500 BC, evidenced in the Ebers Papyrus description of hemorrhoids, varicose veins, and aneurysms. Vessel malformations were apparently also familiar throughout the Roman and early Arabic empires. However, the first modern documented AVM procedure was performed by Hunter during the eighteenth century, the first official clinical diagnosis was described in 1895, and the first surgery (a feeding artery ligation) was performed by Giordano in 1889 and was immediately followed by a full AVM resection in Paris [23]. The early era of AVM microsurgery is traditionally attributed to Kunc et al. who suggested the whole AVM obliteration as a standalone fully efficient treatment strategy in 1965 [35]. The interventional radiology approach and radio-surgical techniques emerged during the 1980s and eventually paved the way for present treatment modalities. The 1980 s were also pivotal regarding treatment strategies. Up to then, recommendations for AVM treatment were mainly institution/physician based. However, during the 1980s, Spetzler and Martin (based on vast surgical work) developed the first well-established AVM classification and treatment scale. This grading scale is considered the "gold standard" decision-making tool in the field to date [36].

\section{Main diagnosis and imaging tools}

Though AVM prevalence is estimated to reach up to $0.2 \%$ of the population, actual confirmed diagnosis rate is only $\sim 0.02 \%[23,37]$. This significant gap is attributed to the fact that AVMs are congenital pathologies remaining generally mildly symptomatic until clinical presentation appears (unfortunately, this is typically hemorrhagic stroke) [23]. AVMs are mostly diagnosed and confirmed using radiology. The imaging findings also greatly influence treatment evaluations. Two strict AVM diagnostic criteria are: indication of a nidus and clear venous drainage [38]. Angiographic elements evaluated as indications for AVMs' clinical significance, and risk assessments include: arterial supply and venous drainage identification, nidus position, geometry, and size, presence of intracranial hemorrhage, and related pathologies [39]. The presence of aneurysms is associated with $26 \%$ of AVM patients [27].

\subsection{Digital subtraction angiography}

DSA is a vascular mapping technique based on conventional angiography. DSA involves an additional pre-processing step that removes non-vascular anatomical data [40]. Angiography is the gold standard methodology for the characterization and delineation of cerebral AVMs and in the evaluation of cerebrovascular diseases $[41,42]$. The method dynamically displays vascular transitions and is uniquely able to delineate the size and number of feeding arteries and their origins, measure the nidus size and compactness, and evaluate venous drainage locations [42]. In addition to its significant diagnostic role, angiography is the main technique used by interventional radiologists to accurately navigate through the neurovascular environment and assess treatment progression and related events through interventional sessions $[43,44]$. DSA is performed using selective (the catheter used for contrast administration is only advanced to the AVM vicinity) or super-selective (the catheter is further advanced to the AVM feeding arteries) approaches [23]. DSA is increasingly being supplanted by the less invasive computed tomography angiography (CTA) and the X-ray and nephrotoxic contrast-devoid MRA. 


\subsection{Computed tomography}

CT comprises a limited diagnostic tool for unruptured AVMs [45]. Lower density areas may (potentially) indicate the AVM in CT scans [46]. The great contribution of CT scanning in AVM diagnosis lies in its high sensitivity for acute bleeding detection, which makes it a front-end diagnostic tool when patients present acute symptoms related to the pathology [47]. CT is also used to assess efficacy and complications of embolization interventional treatments [47].

\subsection{Computed tomography angiography}

CTA combines the CT imaging procedure with contrast agent injection. This enables real-time (RT) structural presentation of neurovascular angioarchitecture (e.g., nidus, arterial feeders, and draining veins) and identifying associated pathologies such as fistulas and aneurysms [38]. Gupta et al. demonstrated that it is even possible to obtain good quality intranidal angiograms using RT CTA for anatomic localization of a specific catheterized (embolized) AVM region [48].

\subsection{Magnetic resonance imaging}

Smith et al. found MRI which is superior to CT and angiography in determining nidus size and location, detecting AVM effects on adjacent brain tissue, and showing the obliteration extent following embolization [49]. MRI also facilitates AVM classification into base categories (e.g., sulcal/gyral) [23]. Use of flow turbulence and velocities as benchmarks has been shown to increase MRI diagnosis efficacy $[23,50]$. MRI is considered highly efficient in detecting and delineating hemorrhagic episodes [23]. In addition, it can detect damage and atrophies in surrounding brain tissue and related pathologies such as aneurysms and fistulas. The latter are considered challenging for early detection and feature high annual risks for hemorrhagic events $(7.5 \%)$ [41].

\subsection{Functional MRI}

fMRI is used to detect abnormalities in brain areas adjacent to AVM borders or deficits in specific cognitive tasks correlated with regions affected by it [41]. fMRI can help to evaluate AVM margins and aid in preventing damage to surrounding brain tissue during interventional sessions. AVMs are congenital pathologies. Thus, it is suggested that the developing brain neuroplasticity will present different fMRI images for healthy and pathologic cases. Caramia et al. used fMRI and transcranial magnetic stimulation (TMS) - which directly explore cortical neurons' electrical activity-and showed cortical motor areas reorganized in the AVM containing hemisphere [51]. The main limitation of fMRI is that it does not directly detect AVMs as MRI does. Therefore, it should be used as a treatment planning tool rather than for standard diagnostics [23].

\subsection{Magnetic resonance angiography}

MRA is valuable in providing AVMs with 3D angiographic images and hemodynamic data [23]. MRA techniques are characterized by two main parameters-flow and contrast. State-of-the-art MRA techniques are:

- Time-of-flight (TOF) MRA: TOF-MRA or inflow angiography enhances blood flow areas by shortening the MRI echo time (TE) parameter, thus reducing the 
execution number in each flow-related voxel in comparison with stationary voxels. This results in flow patterns appearing brighter. The technique can also aid in finding residual AVMs [41]. However, Lee et al. report the accuracy and specificity rates of only $75-78$ and $68 \%$ respectively, which leaves DSA at the front-end [52].

- Phase contrast (PC) MRA (Figure 4): In PC-MRA, phase shift differences between stationary tissue and flowing blood results from the application of a bipolar phase-encoding gradient and a velocity-encoding factor [23]. Since phase change is coupled with velocity vectors, the technique is considered superior to TOF-MRA (better detecting flow directions, minor velocity changes, and slow regions). Chang et al. used PC-MRA post-processing computational fluid dynamics to calculate shear stresses at vascular walls [22]. Wu et al. used PC-MRA to identify peak flows, velocities, and their exact location within the nidus and feeders [53]. To conclude, PC-MRA is a promising technology but is still not comparable with conventional angiography [23].

- Time-resolved imaging of contrast kinetics (TRICKS) MRA: Here, continuous imaging is employed during the passage of a contrast agent yielding a movie with an acquisition rate of up to three frames per second [41]. These improvements enable the visualization and calculation of flow through regions of the AVM (also selectively), enabling confirmation of suspected findings on conventional imaging tools [41]. The technique findings correlate well with DSA with regard to nidus size and location, venous drainage pattern, and arterial feeders in multiple studies but still lag DSA in spatial and temporal resolutions.

\subsection{Diffusion-weighted MRI}

These methods are based on the diffusive anisotropy of water molecules along nerve axons and plexus, where axial motion takes precedence [41, 54]. Here, we can find techniques such as diffusion tensor imaging (DTI), diffusion spectrum imaging (DSI), and high angular resolution diffusion imaging (HARDI). These more recent techniques serve as a valuable non-invasive pre-operative and preradiosurgery tool to evaluate the involvement and proximity of cerebral AVMs to white matter tracts [41]. Using these methods, it provides clinicians with strong risk assessment capabilities, particularly regarding the microsurgery approach and track planning [55]. dwMRI can be used with other technologies for presenting a combined vascular and neuroanatomical image [55]. It might also enable very early identification of ischemic areas [46]. dwMRI is limited mainly to white matter tracts and presents high sensitivity to protocol operation parameters (e.g., TR/TE/ spatial resolution) and artifacts from inadvertent sources.
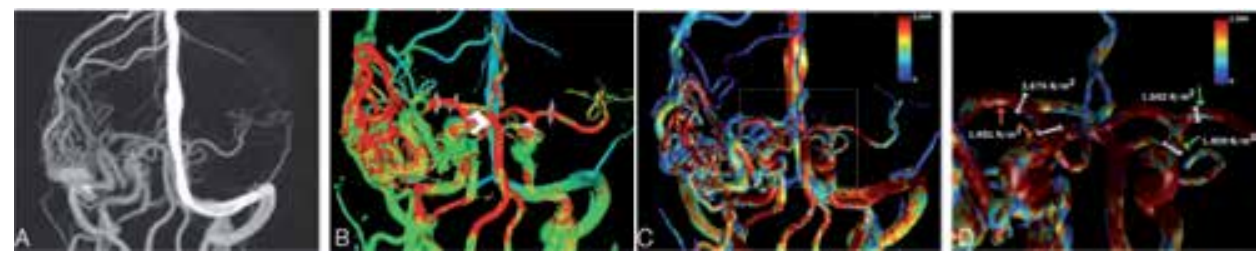

Figure 4.

(A) PC-MRA original image of right temporal AVM, with (B) velocity, $(C)$ whole brain, and (D) feeding arteries shear stresses data [22]. 


\subsection{Summary}

AVM analysis is based on the findings of multiple modalities - vascular anatomy of nidus and feeders, acute/chronic brain matter pathological presentations, flow and velocity rates, and surrounding tissue anatomical and functional attributes (e.g., connectometractography). Each modality presents specific strengths and weaknesses. CT is extremely sensitive to hematomas and offers several AVM anatomical finding capabilities. CTA is used to diagnose AVM in patients with acute hematomas. MRI presents improved topography and the detection of subacute and chronic hematomas and surrounding brain tissue pathologies. PC-MRA is used for high-resolution vascular imaging and provides biomechanical data (e.g., shear stresses), that is, highly beneficial in clinical assessment. Functional-anatomical relations data are effectively gathered using fMRI and dwMRI which provide strong clinical decision-making tools. Finally, DSA remains the gold standard for both diagnostic and procedural purposes due to its high-resolution real-time imaging of cerebral angioarchitecture. To conclude, brain imaging is a prolific field constantly presenting the development of promising improved and new modalities.

\section{Grading and classification}

\subsection{The SM grading system}

In 1986, Spetzler and Martin published a relatively simple AVM classification system called "SM Grading" [36]. SM is considered as the gold standard to date. The system requires three parameters, evaluated using angiography, CT, and MRI (Table 1): size, venous drainage pattern ("superficial" if all drainage is via cortical veins and "deep" if some or all drainage is via deep veins), and neurological eloquence of adjacent brain regions ("eloquent" regions are those well-defined by a neurological function, while the regions of less defined function or disabling effects when disrupted such as temporal lobes and cerebellar cortex are considered “non-eloquent”). SM eventually sorts AVMs into five grades. Inoperable AVMs are considered grade six. Spetzler and Martin correlated their grading with the surgical outcomes of 100 patients and found it to be well-correlated with both minor and major post-operative neurological deficits. Further validations were later performed by both the authors and peers [56].

\subsection{Modifications of SM}

Several modifications of the SM grading system have been suggested over the years. Following are some key examples. De Olivera et al. suggested including

\begin{tabular}{|c|c|c|c|c|c|c|c|}
\hline \multirow[t]{2}{*}{$\begin{array}{l}\text { Graded } \\
\text { feature }\end{array}$} & \multicolumn{3}{|c|}{ AVM size $(\mathrm{cm})$} & \multicolumn{2}{|c|}{$\begin{array}{c}\text { Eloquence of adjacent } \\
\text { brain }\end{array}$} & \multicolumn{2}{|c|}{$\begin{array}{l}\text { Pattern of venous } \\
\text { drainage }\end{array}$} \\
\hline & $\begin{array}{c}\text { Small } \\
(<3)\end{array}$ & $\begin{array}{l}\text { Medium } \\
(3-6)\end{array}$ & $\begin{array}{l}\text { Large } \\
(>6)\end{array}$ & $\begin{array}{c}\text { Non- } \\
\text { eloquent }\end{array}$ & Eloquent & Superficial & Deep \\
\hline $\begin{array}{l}\text { Points } \\
\text { assigned }\end{array}$ & 1 & 2 & 3 & 0 & 1 & 0 & 1 \\
\hline
\end{tabular}

Table 1.

Determination of AVM grade [36]. 
two Grade 3 subgroups with different patient management [57]: 3a (large size, pre-operative endovascular embolization followed by microsurgical resection) and $3 \mathrm{~b}$ (venous drainage and/or eloquence, generally treated with radiosurgery). They found their modified classification a useful guide for the best treatment but indicated that it has many exceptions. Lawton et al. suggested further subcategorizing Grade $3 \mathrm{AVMs}$ into four classes for better correlating the SM grading with associated surgical risks (- less risk, + more risk) [58]: Grade 3-AVMs (small nidus size, deep venous drainage, and eloquent adjacent brain tissue) have a surgical risk similar to that of low grade AVMs and can be safely treated with microsurgical resection, Grade 3+ AVMs (medium size, superficial drainage, and eloquent) have a surgical risk similar to that of high-grade AVMs and are best managed conservatively. Grade $3 \mathrm{AVMs}$ (medium, deep, and non-eloquent) have intermediate surgical risks and require judicious selection for surgery. Grade $3^{*}$ AVMs (large, superficial, and non-eloquent) are either exceedingly rare, with a surgical risk that is unclear, or theoretical lesions with no clinical relevance. Finally, fairly recently in 2016, Neidert et al. suggested a grading system for patients with ruptured AVMrelated IntraCerebral Hemorrhage (AVICH) to predict clinical outcome [59]. Their system extended SM with parameters such as age, diffuse nidus (from the LawtonYoung grading system, that added patient age, hemorrhagic presentation, nidal diffuseness, and deep perforating artery supply in 2010 to improve neurological outcome prediction and refine patient selection [60]), intracerebral hemorrhage volume (30 CC threshold), and intraventricular hemorrhage (derived from the intracerebral hemorrhage score). They demonstrated that their score predicts the outcome of patients with ruptured AVM and associated ICH better than previous grading systems (SM included). They cautioned that an external validation is needed before this score is tested in a prospective multicenter cohort. To date, no modified grading system has become as well-established as the SM grading.

\subsection{SRS grading scales}

Schwartz et al. tried to predict the AVM obliteration success of single-dose photon SRS for individual patients [61]. They defined the obliteration prediction index (OPI $\equiv$ marginal dose of radiation given at the edge of the target lesion in gray/lesion diameter in centimeters). They concluded that the exponential function $P=1-\mathrm{A} e^{-\mathrm{B} \cdot \mathrm{OPI}}$ (where $P$ is the obliteration probability and $\mathrm{A}, \mathrm{B}$ are constants) is well-correlated with successive chance, partly describes the biological effect of radiation, and is independent of the device (marginal dose) used. They suggested that radiosurgery centers use this model to facilitate successful treatment prediction.

Pollock-Flickinger developed a grading system to predict excellent patient outcome (complete AVM obliteration without any new neurological deficit) following single session AVM radiosurgery [62]:

$$
\begin{aligned}
\mathrm{AVM}_{\text {score }}= & \left.0.1 \mathrm{AVM}_{\text {volume }}\left[\mathrm{cm}^{3}\right]+0.02 \text { Patient age [years }\right] \\
& +0.3 \mathrm{AVM}_{\text {location }}^{*}
\end{aligned}
$$

where ${ }^{*}$ indicates: frontal or temporal $=0$; parietal, occipital, intraventricular, corpus callosum, or cerebellar $=1$; basal ganglia, thalamic, or brainstem $=2$.

They concluded that their proposed AVM grading system strongly correlates $\left(R^{2}=0.88\right)$ with patient outcomes but cautioned that further testing by independent centers using prospective methodology is still required.

In 2016, Pollock et al. compared five AVM grading scales-SM, radiosurgerybased AVM score (RBAS), Heidelberg score, Virginia Radiosurgery AVM Scale (VRAS), and proton radiosurgery AVM scale (PRAS) — at predicting SRS outcomes 


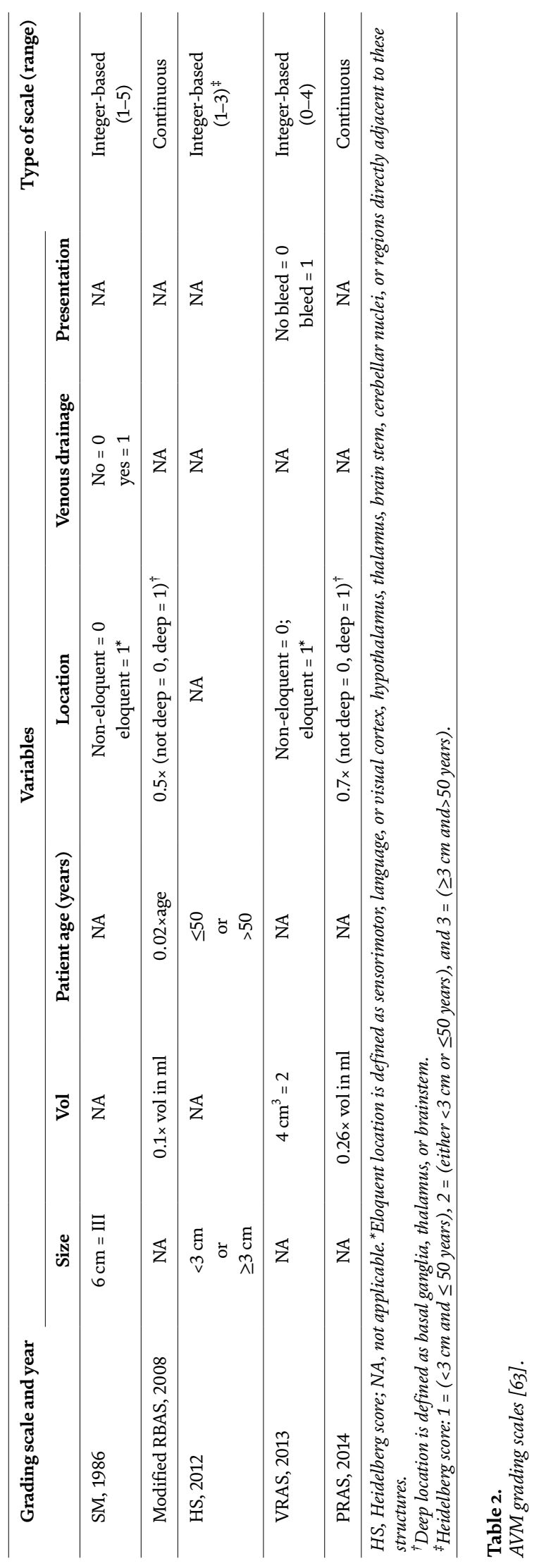




\begin{tabular}{lcc}
\hline AVM feature & & Points \\
\hline Number of feeding vessels & $<3$ & 1 \\
\cline { 2 - 3 } & $3-5$ & 2 \\
\cline { 2 - 3 } & Non-eloquent & 0 \\
\cline { 2 - 3 } & Eloquent & 1 \\
\hline Presence of AVF & No AVF & 0 \\
\hline${ }^{a} A V F=$ arteriovenous fistula or fistulous component. & AVF & 1 \\
\hline
\end{tabular}

Table 3.

Classification scheme for risk assessment during embolization procedures for brain AVMs [64].

(Table 2) [63]. Their criterion was AVM obliteration without a decline in modified Rankin Scale (mRS) score (excellent outcome). They concluded that continuous scores AVM grading scales (RBAS and PRAS) outperformed integer-based grading systems in the prediction of AVM obliteration outcomes since they directly correlate with patients' existing physical attributes.

\subsection{Grading systems for embolization}

Even though endovascular embolization widely differs from surgical or SRS approaches, dedicated grading was not considered as a broad/general tool until fairly recently. In 2010, Feliciano et al. conducted an extensive literature survey and correlated endovascular treatment with AVM characteristics. Points were given according to feeding vessels, eloquence, and fistulae presence (Table 3) [64]. They concluded that a grading scale similar to SM for use in risk assessment and outcome determination in brain AVM patients treated by endovascular techniques seems adequate and clinically feasible.

\subsection{Summary}

Though posited in 1986, the SM AVM grading system remains the gold standard in predicting surgical treatment success. The development and assimilation of SRS led to uniquely dedicated grading. The future probably lies with "continuous" grading, where scores are directly correlated with AVM and patients' actual features and properties. Endovascular dedicated grading has just recently emerged, mostly based on large literature surveys and meta-analyses, but apparently shows real promise (though it still necessitates firmer actual validation).

\section{Embolization}

Embolization is intended to physically block blood flow to the AVM. It is a minimally invasive endovascular procedure carried out by an interventional radiologist. AVM embolization is considered among the most challenging in the field due to the vasculature target tortuous hemodynamic formation but, more so, due to its high-pressure arteries directly connecting with low-pressure veins (AV shunts). If arteries are proximally occluded, anastomoses develop from nearby vessels creating new shunts. Thus, proximal arterial occlusion has no curative effect and is restricted to pre-surgical situations [23]. In contrast, direct AVM treatment requires 
distal (transarterial) embolization. First, navigation is performed all the way to the venous section. Then, an embolic agent is super-selectively introduced into the draining veins via microcatheters that are retracted backwards as the vasculature fills up (all the way to the arterial feeders). Vessel selection tract is traditionally based on DSA.

In 1995, Frizzel et al. reviewed the cure, morbidity, and mortality associated with the embolization of 1246 brain AVMs during the previous 35 years [65]. Cure rates were $4-5 \%$. Temporary and permanent morbidities were 10 and $8-9 \%$, respectively. Mortality was 1-2\%. These statistics improved over the years. However, to date, embolization is generally considered a pre-operative (pre-SRS) adjunctive procedure because: (I) as a sole modality, it is assumed effective only in a minority of cases [66]; (II) proximal occlusion of feeding arteries appears to be associated with recurrence [66]; and (III) it appears to increase hemorrhagic risk compared with conservative management, especially in unruptured AVMs [67].

Currently, the two most common embolization agents in cerebral AVM treatment are $N$-butyl cyanoacrylate (NBCA) and ethyl-vinyl alcohol copolymer (EVOH)-DMSO solvent (Onyx) $[68,69]$. These materials are delivered in liquid form and are, hence, injectable through very narrow diameter microcatheters.

\subsection{Cyanoacrylates}

Cyanoacrylates solidify by polymerization initiated once they contact an anionic environment such as blood [69]. The process is very rapid but can be delayed by dilution using Lipiodol (Ethiodol in the USA) vehicle retardant. The more Lipiodol the mixture contains, the longer the delay. Optimizing dilution is a very empirical process that greatly depends on operator experience level. Cyanoacrylates' main advantages are that they: facilitate nearly instant occlusion; induce an inflammatory response within the embolized vessel walls that are believed to play an important role in the occlusion durability; are compatible in case of vascular rupture; are injectable via many microcatheter types (even the thinnest and most flexible ones currently available); have a non-glued microcatheter withdrawal that gives rise to minimal vascular network traction, so they are highly compatible with narrow diameter arterial vessels (very sensitive to traction-induced mechanical trauma); and facilitate surgical resection by helping to identify embolized vessels (compressible and easily cut with micro-scissors). Cyanoacrylates' main drawbacks are: catheter can become entrapped in the occluded vessel; difficulty in controlling the occlusion position; highly local occlusion; they can only be used by operators with extensive training; must be opacified to monitor flow during injection; and catheter position must be abandoned.

\subsection{EVOH copolymer-DMSO solvent (Onyx)}

Here, small polymer particles are suspended in solution using a DMSO solvent $[69,70]$. Following mixture injection, DMSO diffuses to surrounding tissue, resulting in particle aggregation occluding the lumen. Flow is omnidirectional and typically includes artery reflux along the microcatheter tip. Following injections progressively colonize adjacent arteries, only then traveling towards the draining veins. Consequently, microcatheter tip trapping is a typical feature. This led manufacturers to develop catheters with detachable tips. Onyx's main advantages are: relatively high complete obliteration rate with the evidence of stability over time [70]; slow solidification facilitates prolonged/controlled injection with deeper nidus penetration; and mid-procedure angiography and reduced catheter adherence even during reflux. Onyx's main drawbacks are: DMSO is toxic_rapid injection can 
cause vasospasm, necrosis, and acute respiratory distress syndrome (ARDS) [71]; DMSO-compatible catheters and syringes must be used; high radiopacity causes poor visualization during reflux in very small vessels and masking by previously embolized regions potentially leading to subsequent healthy vasculature embolization; over-reflux can potentially harm adjacent functional healthy arteries; and mixture must be shaken for at least $20 \mathrm{~min}$ prior to usage in order to homogenize the tantalum powder used for opacity.

\subsection{Precipitating hydrophobic injectable liquid}

PHIL is a recent liquid agent composed of a non-adhesive copolymer (polylactide-co-glycolide and polyhydroxyethylmethacrylate) dissolved in DMSO. Triiodophenol is used as an iodine component, being covalently bound to the copolymer for radiopacity [72]. Initial studies show embolization characteristics, embolization extent, and biocompatibility to be comparable with those of Onyx [72-74]. However, further studies are required to fully evaluate its safety and efficacy [74]. PHIL main advantages are: shorter pause times that result in significantly higher embolization success compared with Onyx; lower volumes required for the same extent of embolization compared with Onyx; it comes ready for use (does not require preliminary preparation); and improved visibility compared with Onyx. PHIL's main drawbacks are: still necessitates DMSO; embolization performance (efficacy) is only comparable with that of Onyx but does not improve on it; and could result in the exertion of traction on the vascular network upon catheter extraction.

\subsection{AVM embolization complications}

Post-embolization hemorrhage is the most severe, dramatic, and morbiditymortality-related complication [75]. Up to $14 \%$ of patients exhibit neurological deficits $[75,76]$. The combined death and permanent disabling neurological deficit rate is below 3.9\% per patient [77, 78]. Risk predictors for endovascular treatment differ from those for AVM surgery [76]. Some studies report no morphological AVM characteristics test predict treatment complications [76]. Others suggest AVM location in an eloquent brain part, and fistula presence and a venous glue deposition are associated with complications [77]. Yet, others consider that basal ganglia location is weakly associated with new post-embolization neurologic deficits [78]. This topic is controversial. It appears that extensive devascularization and the absence of post-procedure hypotension increase hemorrhage risk [75]. Thus, partial (25-30\%) devascularization per session and post-procedure hypotension induction were recommended [75]. Overall, there is a consensus that brain AVMs' embolization is associated with low overall mortality and disabling morbidity rates [77, 78]. The hemorrhage mechanisms are typically: artery perforation by a microguide/ microcatheter during navigation; excessive pulling on a stuck microcatheter; and hemodynamics-related rupture due to changes in flow patterns and commination (size reduction) in venous drainage. These are typically the most severe and occur within $48 \mathrm{~h}$ following embolization. Finally, thrombus formation and its migration from the carrier catheter leading to ischemic complications is a feasible though non-frequent scenario.

Embolization complications dictate clear design recommendations for future endovascular devices: gradual blood vessel closure in which no abrupt flow changes take place inside the nidus; the ability to treat small and big blood vessels, easier to operate when treating patients-requiring reasonable training and experience (and skills); and avoiding exerting significant mechanical stresses on the delicate vasculature. 


\section{Stereotactic radiosurgery}

SRS is a leading alternative to surgery or embolization, especially for AVMs located in deep or eloquent brain regions, where invasive treatment is not optional [79]. SRS employs ionizing radiation for gradually occluding AVM blood vessels. Its application was adopted from oncology during 1970-1980s [80, 81]. During the next decades, SRS (or "gamma-knife") rapidly evolved as a standalone modality and following embolization. SRS systems typically comprise a spherical array of high-focused gamma ray generators (Figure 5), a mechanical system that precisely positions and immobilizes patients' heads and a 3D imaging, and tracking system for treatment (i.e., dose delivery) design and real-time management (Figure 6). The chief benefit of radiosurgery is that it can eliminate the threat of spontaneous intracranial hemorrhage by gradual obliteration of the AVM over 2-3 years [81].

\subsection{Histopathological response}

Radiation induces endothelial damage (lasting biochemical changes and apoptosis), thrombocyte aggregation/development of fibrin microthrombi, and subendothelial/ perivascular spindle cell proliferation (contractile myofibroblasts formed in vascular walls and AVM connective tissue-stroma) [23, 82, 83]. Both degenerative and proliferative changes are dose- and time-dependent. Degeneration expressions are tissue granulation and inflammatory cell presence in the stroma followed by type IV collagen-producing fibroblasts and fibrocytes and eventually hyaline phenotypics and obliterated vessels. Proliferation is expressed by the formation and accumulation of myofibroblasts (neointima) assumed as the canonical shrinking and occlusion factor in irradiated AVMs [23, 82, 83]. Importantly, normal vessels do not exhibit propensity to obliterate. Apparently, due to the connective tissue (stroma) surrounding the AVM nidus, pathological vessels playing a key role in the obliteration process $[23,84]$. Obliteration is often followed by new vessel formation, occasionally visible on MRI [23, 82]. Radiation-induced necrosis, neural loss, myelin fragmentation, and gliosis have been detected in the surrounding brain tissue 1-10 $\mathrm{mm}$ from the lesion

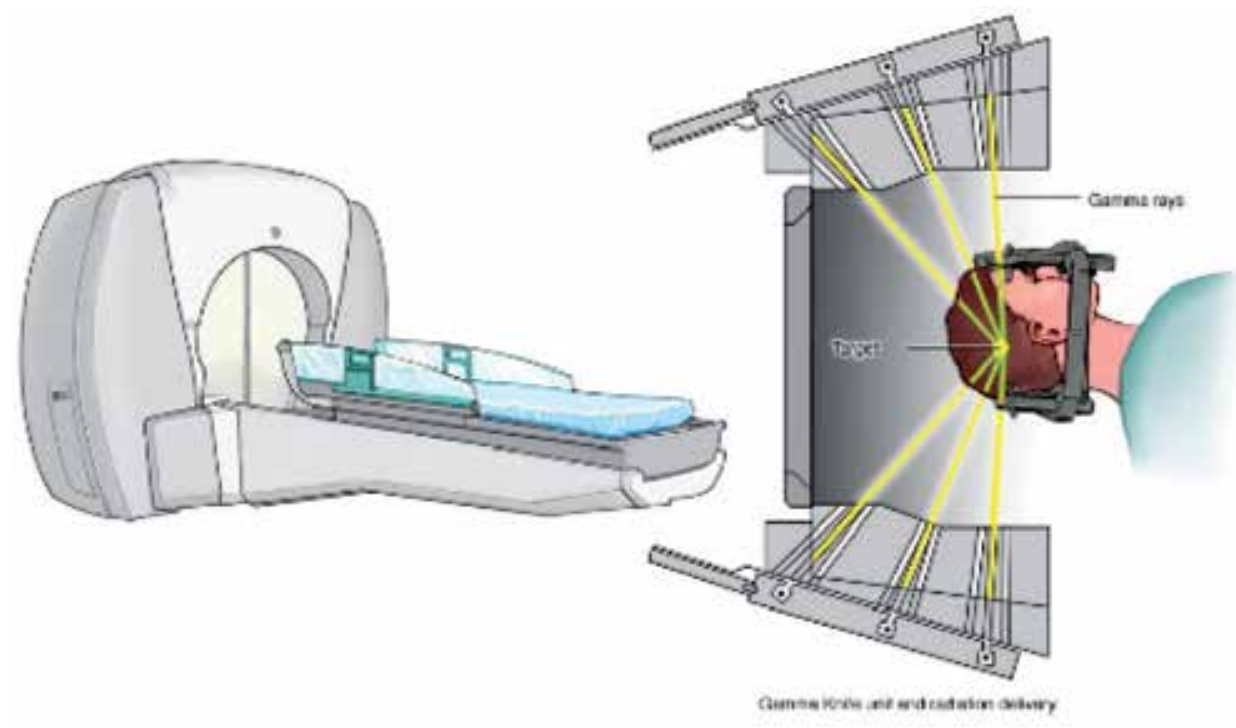

Figure 5.

Schematic illustration of a stereotactic radiosurgery procedure [(C) Mayo clinic]. 

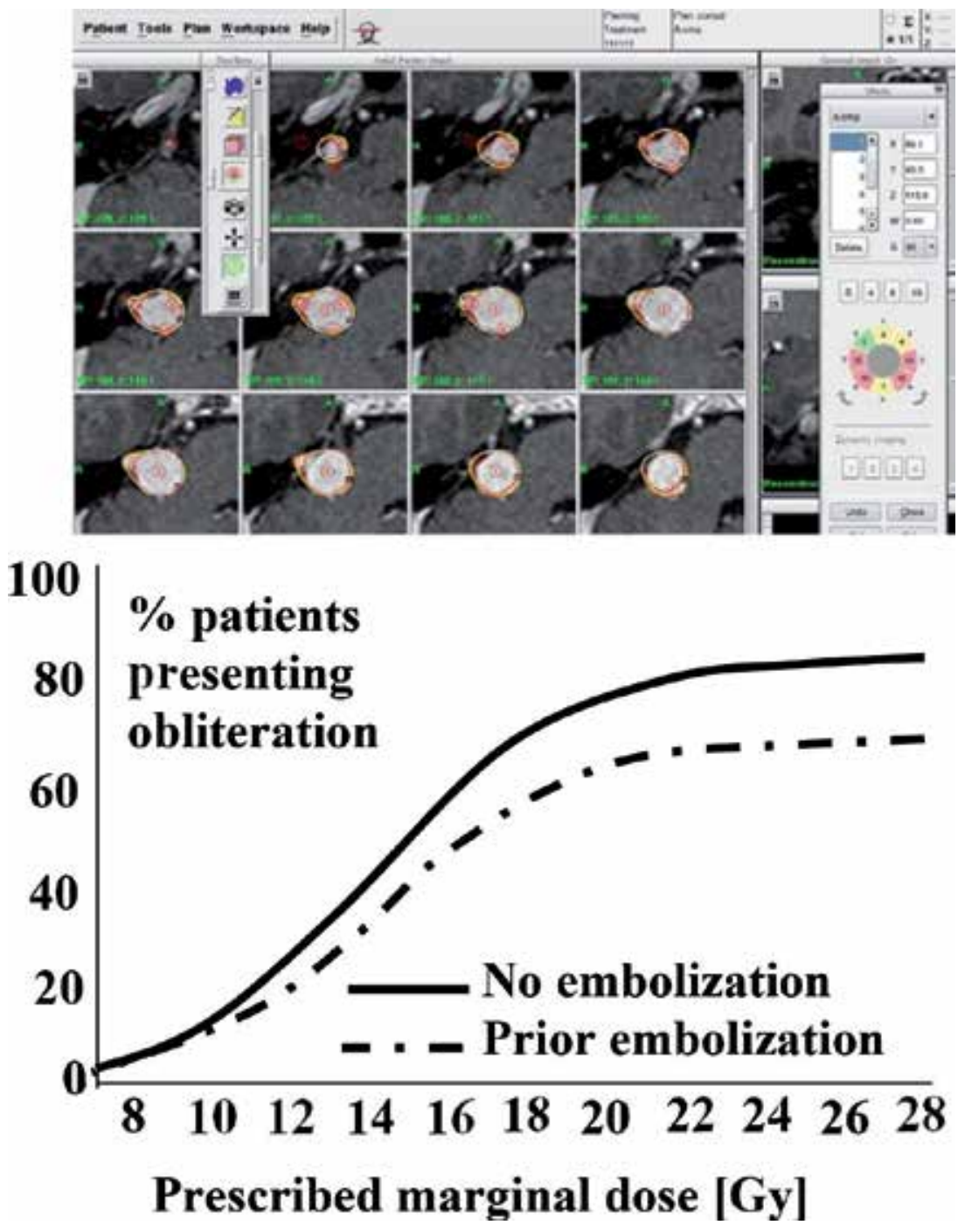

Figure 6.

Top: SRS treatment planning (dosimetry). Using imaging scans and specialized software, the treatment team determines the best combination of radiation beams to target the lesion [C Mayo clinic]. Bottom: marginal dose versus efficacy (following [81]).

border [23]. Histopathology is typically elicited and efficacy-controlled by focused irradiation of marginal doses of $\sim 10-35 \mathrm{~Gy}$ (median 20) (Figure 6) — delivered in a single or fractionated (higher doses) protocol $[81,82]$. It has become a consensus that a better understanding of irradiation response physiology may facilitate the targeting of individual enzyme systems and open up new SRS opportunities [84].

\subsection{Widely used systems}

Gamma-knife systems consist of up to 200 cobalt-60 sources arranged in circular arrays within a spherical mechanism [79]. The arrays' geometry and source strength (typically $\sim 30$ curies each) result in a 3D energy field of isodoses at any defined volume within the sphere. While each source beam dose is very low, the converged irradiation at any pre-chosen focus position adds up to clinical values. The targeted volume can be varied using different sized collimators. These systems 
traditionally required complete patient immobilization-which involves direct mechanical fixation of patients' skulls using a stereotactic frame. The fixation is an invasive procedure that can be highly painful and stressful. Newer models can perform frameless treatment (thermoplastic mask for immobilization and advanced imaging for stereotactic orientation), thus supporting a less invasive patient experience. Gantry and robotic arms systems employ gantry-mounted linear accelerators ("LINACS") to generate the energy beam and rely on either fixed circular or multi-leaf collimators (MLCs) for its shaping. This allows a conformal schemewhich aids in treating irregular nidus geometries. Alternatively, the system can be mounted on a robotic platform (arm vs. gantry) that adds mechanical degrees of freedom. Patient positioning can also be achieved using imaging (cone beam CT) or laser systems, so frame-based immobilization systems are becoming obsolete. Less common are proton beam systems that accelerate protons (using a synchrotron or cyclotron) in order to generate the therapeutic beam. An advantage of proton treatment is the minimal target-exiting dose safeguarding the lesion surrounding tissue. The proton beam is typically delivered to the target volume via a gantry mechanism. Patient immobilization can be achieved invasively and non-invasively. Proton systems' main disadvantage is that they are scarce and expensive.

\subsection{Treatment selection}

Since radiosurgery/therapy equipment is scarce and extremely expensive, many centers and physicians have adopted particular patient selection and treatment protocols. The availability of different systems per hospital/clinic has further contributed to this diversity. The result has been that there are many controversies regarding patient and modality selection in general, and with respect to radiosurgery/therapy in particular [85]. During the last two decades, this trend has been changing as more physicians and researchers are attempting to standardize scorebased radiotherapy grading and patient selection systems (see Section 4.3) [59]. A treatment selection scheme established at Pittsburgh University is presented in Figure 7 [81]. Selection relies on AVM volume and location as well as the existence of post-treatment (residual) lesions.

Unlike SM grading in surgery, to date, there has been no distinct established treatment selection criterion for radiosurgery/therapy. Even recent advanced grading systems are not widely considered a sufficiently standardized basis for designating a patient for radiation modality. Common factors are bleeding history, patient age, existing comorbidities, anatomical location, and clinical history [81].

\subsection{Outcomes and complications}

Standard protocol for AVM obliteration typically involves clinical diagnostics and a half-yearly MRI, followed by annual MRI imaging. Final validation (classically at 3 years) is DSA-based, as the latter constitutes the gold standard [81]. A meta-analysis, performed by Badra et al. in 2018 [79], found that: obliteration rates are $\sim 70-80 \%$ at $2-4$ years post-treatment; annual bleeding rates are $1.1-8 \%$; AREs are $12-38 \%$; and overall morbidity is $4-12 \%$. Another study showed [81]: the obliteration rates of $78 \%$ at 3-year follow-up, repeated radiosurgery needed in $12.5 \%$ of cases, post-surgery annual bleeding rates in $4.1 \%$ of cases, and AREs in $16.3 \%$. Thus, while radiation treatment is relatively effective, it is not risk- and complication-free. Several categories merit particular attention: acute complications are typically related to the post-procedural presentation of neurological deficits after 2 years or more. Yen et al. studied 1426 Gamma Knife Surgeries (GKS) and found radiationinduced imaging changes (RICs, visualized as increased T2 signal surrounding the 


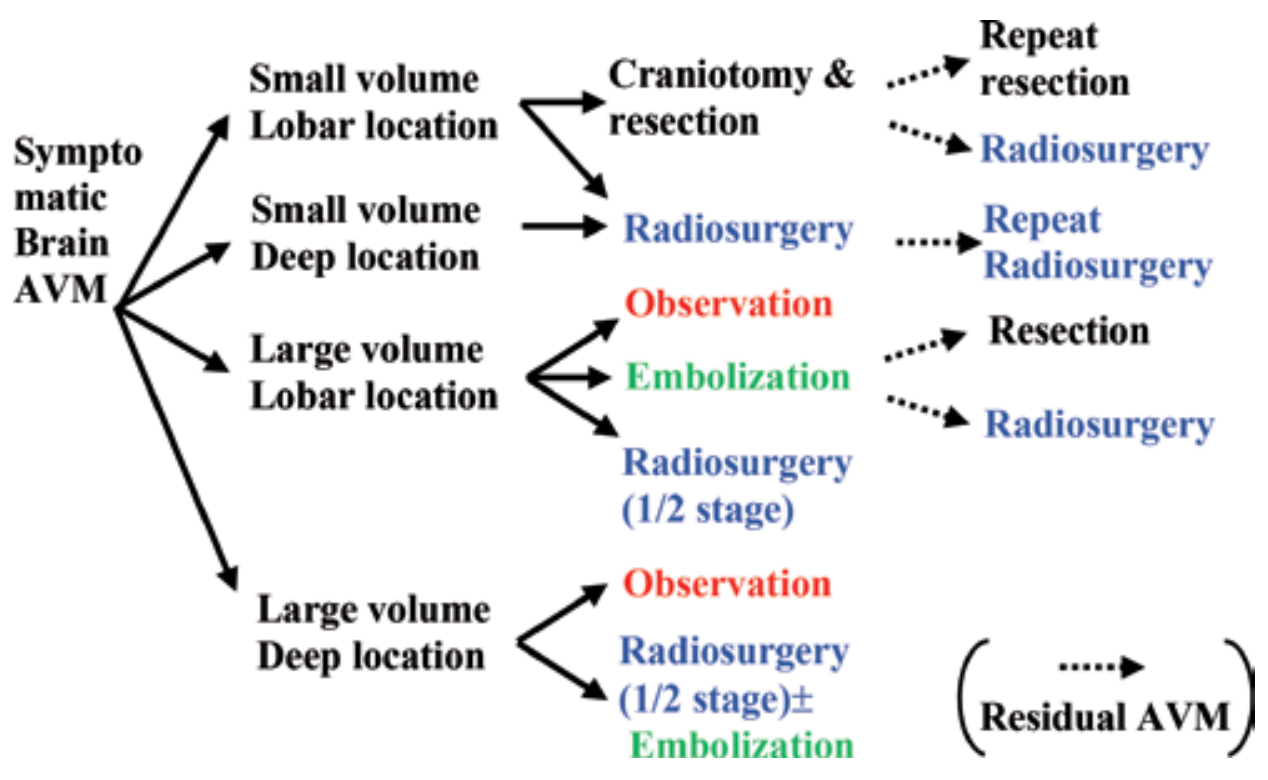

Figure 7 .

AVM treatment selection scheme (following [81]).

treated nidi on MRI) that are the most common adverse effects following GKS-in $33.8 \%$ of lesions [86]. Patients with a relatively healthy brain and large nidi or with a single draining vein were more susceptible to RICs. Few RICs were symptomatic (8.6\%), and most symptoms were reversible (only $1.8 \%$ had permanent deficit). About $7.7 \%$ of RICs were acute-causing a midline shift of the brain. Late AREs typically occur many years following SRS (6.9\% of patients after a median of 8.7 years. The 5-, $10-$, and 15 -year incidence is $0.4,7.7$, and $12.5 \%$, respectively). They are characterized by perilesional edema or cyst formation and are distinct from short-term (1-2 years post-surgery) AREs, often labeled as RICs [87]. About $3.4 \%$ of Late AREs are symptomatic at detection. Many that are asymptomatic later present with cyst progression. The overall symptomatic rate of late AREs is $4.7 \%$ [87]. Another study included radiation necrosis and cystic vessel formations in late ARE diagnosis and found the incidence at 2-6\% [79]. Finally, since radiotherapy for AVM is based on ionizing radiation, the concern for carcinogenicity arises. However, little evidence has been found to support radiation induced tumors (RITs). A late retrospective analysis performed by Pollock et al. found no RITs in 471 patients observed between 1990 and 2009 (with very few others out of the total 1837 patients presenting malignant transformation) [88]. The authors concluded that the risk of RITs or malignant transformation after SRS is very low and should not be used as a justification for choosing alternative treatments. Lunsford et al. corroborate these findings [81].

\subsection{Fractionated radiation/treatment}

FR strategy is intended for treating large lesions that carry increased mortality and morbidity using interventional methods and lower obliteration rates or longer obliteration time when treated using radiotherapy. Unfortunately, large AVM is not a well-defined or accepted criterion in the literature. SM refer to $>6 \mathrm{~cm}$ along the nidus largest diameter. Other studies define a volumetric condition $>10\left(\mathrm{~cm}^{3}\right)$ [62]. However, there is a consensus that high single-fraction doses as used in small AVMs increase radiation injury risks and associated neurological deficits when 
large normal brain volume is exposed to high doses [89]. Two approaches exist to improve safety and efficacy in these cases: (1) fractionation radiosurgery (FR) where total dose is equally divided into fractions delivered over multiple sessions (days scale), so brain tissue adjacent to the lesion can tolerate higher integral doses.

(2) Volume staged radiosurgery where the AVM is typically divided into 2-4 spatial regions each treated separately using a high single-fraction dose. Between treatments, a rest period (3-9 months) is imposed. Here, each AVM part is instantly treated with a clinically effective dose. Normal brain tissue recovers between treatments. This makes it possible to tackle the main disadvantage of FR-low fractional doses are relatively less effective at treating AVM and result in reduced obliteration rates unless the total dose is substantially increased [89]. However, a main concern of this approach is that partial AVM obliteration apparently alters blood flow patterns and increases hemorrhage risks [89]. Currently, there is a little evidence for the superiority of one approach over the other [89]. In general, it is highly recommended to minimize the integral dose to the normal brain of asymptomatic patients. Recently, Unkelbach et al. elegantly demonstrated that by boosting complementary parts of the target volume in different fractions, it may be possible to achieve a therapeutic advantage in FR since this approach facilitates mean dose reduction in the normal brain [89]. To date, FR has not been widely accepted as presenting a compelling advantage over multi-modality treatments such as initial size reduction using embolization.

\subsection{Summary}

While gaining popularity and presenting impressive treatment success rates of $\sim 80 \%$, radiosurgery still has distinct limitations that must be faced. Obliteration typically takes 2-3 years during which patients remain exposed to significant annual bleeding risks of $4-5 \%$ (twice that of conventional treatment) and overall morbidity rates reaching $12 \%$. Large (SM grade $\geq 3$ ) AVMs necessitate multi-modality or fractionated treatment. The latter shows promise but requires further study if efficient management is desired.

\section{Surgical resection}

\subsection{Patient selection and outcome predictors}

The SM grading system for predicting patient outcome is microsurgery-based and remains the gold standard in the field [36]. Though the physiological origins of each AVM's clinical presentation are unknown, SM and supplemental grading systems have been validated on numerous occasions (see above). These systems apply to both patient selection and outcome prediction. Recently, Kim et al. demonstrated, in a cohort study performed on 1009 AVM patients at four institutions, that supplemented SM grades (SM-Supp, also considering the patient age, bleeding history, and compact anatomy indication) have greater predictive accuracy than standalone SM grades [90]. Their findings are not unique in that they show improvement over SM. They also rightfully conclude that "current grading systems are imperfect and evolving and that as the pathophysiology, hemodynamics, and genetics of AVMs are elucidated through research, grading systems will incorporate these advances." We, however, believe that there is a great difference between academic and clinical settings. We acknowledge that complex grading schemes may present improved predictability and selection capability. However, we believe practitioners must be provided with simple, intuitive, and relatively easy-to-use schemes. Only 
such schemes will be readily accepted and rapidly/easily implemented in clinical settings, particularly in emergency cases. We, therefore, recommend that the factors incorporated into future grading schemes remain relatively accessible (direct) and limited in number.

\subsection{Standard microsurgery protocol}

We briefly (and humbly) review key steps involved with AVM microsurgery $[23,37]$. Once a patient is designated for microsurgery, treatment planning and evaluation begins. Often, additional imaging is performed to provide maximal data for a solid operating plan. Here, 3D reconstruction can prove very helpful and emphasis should be given to study the AVM main feeders. Pre-operative steps include patient positioning (one that provides a good venous return and preferably has the AVM surface aspect horizontal at the top of the approach and the longest axis of the nidus vertical) and craniotomy (removal of part of the skull to expose the brain), which provides exposure to the lesion area. Here, caution must be exerted to ensure the craniotomy is larger than the AVM surface while safeguarding the delicate dilated and exposed draining veins during dura opening (against adhesion). Only then can AVM resection be performed. The procedure in general is directed from the arterial towards the venous side of the nidus. All vessels must be coagulated or clipped. It is customary to leave true feeders to the end since their earlier occlusion can lead to the recruitment of blood flow from deep feeders and may cause their rupture [23]. It is important to ensure that draining veins present no evident flow. Eventually, the nidus is gently rolled out of the resection cavity. Post-operative management includes admitting the patient (while completely sedated) to the Intensive Care Unit for close observation for evidence of postoperative bleeding or swelling. A post-operative CT is typically performed shortly after the procedure to rule out any such complication followed by a mandatory angiography performed on day $2-3$ after surgery.

\subsection{Results and complications}

The main advantage of AVM microsurgery is its "straightforward" approach, which allows relatively definite lesion resection and rapid clear follow-up. Microsurgery is considered the gold standard in AVM obliteration. It presents a high success (cure) rate reaching 99\% in small SM 1-2 grades [91-93]. Treatment challenges include limitations regarding accessibility (deep locations) and a high risk of severe complications that contribute to the mortality and permanent morbidity rates of 3.3 and $8.6 \%$, respectively, as seen in a meta-analysis performed in 2425 patients between 1990 and 2000 [8]. A more recent study found permanent mortality and morbidity rates of 1.7 and 4.8\% (2.2\% permanent significant morbidity) [91], and yet, another study found the permanent mortality and morbidity rates of 7.9 and $14.8 \%$ (though obliteration rate was $87.2 \%$ ) [92]. Additional studies present early and permanent disabling deficits in 12.3 and $4.5 \%$, respectively, permanent neurological deficit in $16.1 \%$ [93], perioperative neurological deficits of $17 \%$, annual hemorrhage rate of $0.3 \%$, and a recurrence rate of $0.9 \%$ in children [94]. These findings and others clearly demonstrate the pronounced variations in the modality outcomes depending on surgeons' expertise, patients' AVM grading distribution (e.g., 7\% neurological deficits at SM Grade 1 compared with $50 \%$ at SM 5 in [90]), prior bleeding presentations, multi-modality protocol (e.g., surgery following embolization or radiosurgery as in [95]), and so forth. A review of the literature indicates that the leading risks of surgical resection are intraoperative rupture, post-operative hemorrhage, and post-operative edema. These hemodynamic events can become life threatening and disabling. Additional 
complications of reduced risk are neurologic deficits from over-dissection or ischemia, seizures, hydrocephalus, and infections [23, 37]. The subject of lesion recurrence following microsurgery has been controversial. Different centers report highly varied outcomes. Recently, Aboukaïs et al. analyzed the subject and reported the recurrence as a fairly rare case (7/138 cases) affecting mostly pediatric patients. They recommend particularly long-term angiographic follow-up in children to detect AVM recurrence or remnants [96].

\subsection{Emerging trends}

Brain microsurgery is a proliferating field presenting many interesting developments in imaging, treatment management, surgical approach, and so forth. We very briefly mention a few promising directions. Molecular imaging employs specific antibodies combined with detectable agents such as gadolinium [55]. This technique was used to image particular receptors on tumors and could non-invasively detect biological markers in AVM vessels. If an appropriate biomarker imaging probe for AVM is discovered, it will facilitate highly selective lesion diagnosis and analysis. Specific conditional biomarkers (activated by physiological occupancy of enzymes, ions, and metabolites) can possibly even support super-selective procedures. Rad et al. demonstrated that vessels within the mature rat AVM exhibit elevated phosphatidylserine (PS) externalization compared with normal vessels [97]. Ionizing radiation increased PS externalization in a time-dependent manner. They concluded that the AVM localization of PS externalization may function as a tool in future SRS treatment. Image guidance provides a promising technique, particularly when incorporated into patient intraoperative 3D viewing and simulating systems [98]. It facilitates improved AVM localization, clearer venous anatomy, better definition of craniotomy, and so forth for the surgeons, and may reduce intraoperative risks. Spetzler and Sanai used dynamic retraction (retractorless surgery) and a variety of advanced handheld instruments with considerable success and suggest that fixed retraction can be supplanted by this approach, thus limiting the risk of retractor-induced tissue edema and injury [99]. There are additional promising directions outside the scope of this chapter. However, most of those mentioned (as well as those not discussed here) still lack a large enough database for establishing clinical superiority.

\subsection{Summary}

The neurosurgical aspect of AVM treatment presents a versatile picture. While SM Grade 1-2 lesions are treated with good efficacy and low risks (as well as a few Grade 3 cases), successful outcome rapidly declines in medium-to-large lesions, demonstrating high risk for adverse outcomes (transient and permanent neurological deficits as well as mortality). These high risks do not markedly decline when addressing large AVMs using a multi-modality approach. Perhaps because such procedures are not yet sufficiently established, we cannot currently provide this statement with distinct supporting evidence. Small AVMs constitute a large portion ( $40 \%$ or so based on our literature observations) of all lesions. This leaves many cases without direct surgical solutions and greatly limits the use of this approach as a single modality. Future developments include further advancements in imaging methods, augmented reality and simulation systems, and innovative tools and approach methods. These will certainly facilitate continuous improvement in efficacy and safety with regard to small lesions and an increased ability to effectively treat medium lesions. However, we fear that treating the majority of medium-to-large lesions will continue to necessitate multi-modal, fractionated, or novel approaches. 


\section{Discussion and recommendations}

The ARUBA trial, published in 2014, compared interventional therapy with medical management of unruptured brain AVMs. It was a broad trial involving 39 active clinical sites in nine countries. The study recruited 223 patients during the period from April 2007 to April 2013. About 114 of the patients were assigned to interventional therapy and 109 to medical management. The conclusions were that the risk of death or stroke is significantly lower in the medical management group than in the interventional therapy group (hazard ratio 0.27 ). Naturally, the study elicited a plethora of reactions that we will not fully cover since they are outside the scope of this review. Some, that were relatively more supportive, identified ARUBA as the only randomized trial at that time (2010) with clear clinical outcomes comparing different interventional treatments for brain AVMs with conservative medical therapy [100]. More reactions were less supportive, criticizing the pragmatic design, the patients' heterogeneity, the lack of standardization of the treatment arm, the choice of outcome measures, the short follow-up period, the small population, and so forth $[87,101,102]$. The controversy also led the European societies dealing with the treatment of AVMs to conduct a consensus conference at the European level [104]. Among the statements made were two key points which we quote: "There are sufficient indications to treat unruptured AVMs Grade 1-2 SM" and "There may be indications for treating patients with higher SM grades, based on a case-to-case consensus decision of the experienced team." One clear consensus emerges-further research is advocated to delineate the optimal management of unruptured AVMs, particularly those with SM grade $\geq 3$ [103]. Furthermore, judicious observation of the literature since ARUBA indicates that there may be a lacuna or at least weakness in interventional modalities when addressing high SM grade AVMs. This fact has not traversed the community unnoticed. All three main interventional modalities present a similar line-advancement in existing treatment paradigms, treatment planning, and intra-operative measures. However, to date, the core challenge persists. We feel that one possible cause is the limitations inherent in present approaches that lead to diminishing returns with every new improvement (necessitating ever-increasing technological and financial investments). The two leading avenues of interventional choice for medium-to-large lesions are currently multi-modal and staged treatment. When considering multi-modal treatment, we must take into account other factors besides medical outcomes. Multi-disciplinary AVM treatment suffers from a fundamental market/commercial flaw. Every manufacturer focuses on its core technologies (whether they are embolic agents, radiation therapy equipment, etc.), and these typically do not complement one another. This considerably impedes R\&D of novel multi-modal techniques and protocols by leaving them in the hands of mostly research endeavors that lack the financial resources of commercial companies. Interestingly, this fact is clearly reflected in the literature. The number of papers we reviewed dealing with single modality treatment is an order of magnitude larger (speaking cautiously...) than those adopting a multi-modal approach. This trend continues for the number of patients treated. To conclude, we fear that multi-modal treatment faces inherent financial and technical limitations that strongly impede its chances of reaching full potential and will continue to do so in the near future. Fractionated radiation and multi-session (staged) embolization also suffer similar logistic and economic flaws. It is very challenging to repeatedly admit patients to very complex and expensive procedures also requiring highly experienced medical experts who are typically in "short supply," and advanced facilities, particularly in radiation treatment. Considering this state of affairs, we conclude that it could be advantageous to consider a treatment approach that has not been used to address AVM in the past-continuous mild 
irradiation provided via an implantable active source. The use of such implants in Brachytherapy is very well established and dates back decades (if not a century). Much knowledge has been accumulated in the field (we will not review the subject due to lack of space). Such an implant has the potential to elicit a hyperproliferative effect facilitating lumen closure by thickening of the vascular wall by exploiting the "candy wrapper" or edge effect (see further data below).

First, let us attempt to convince the readers that this approach merits medical investigation. In Section 6.1, we explained that there are two major changes in tissue when exposed to radiation-degeneration and proliferation. Both changes are dose- and time-dependent. Therefore, there is room for adjusting and augmenting each by controlling radiation kinetics and spatial distribution patterns. Active stent studies show proliferation and restenosis reduction (typically in-stent) but also induction (typically at the stent edges). Albiero et al. implanted 122 32P radioactive $\beta$-emitting stents (activity levels of $0.75-12.0 \mu \mathrm{Ci}$ ) in 91 lesions in 82 patients [105]. After 6-month follow-up, they found that intrastent restenosis was $0-16 \%$ (depending on implant activity; high activity stents showed no restenosis). However, they also found that restenosis at stent edges was 41-52\% (maximal for the lowest activity stents!). They concluded that the use of active stents in patients with coronary artery disease is feasible and named this edge effect as the "candy wrapper." They speculated that the effect was a result of low radiation at the stent edges combined with an aggressive approach to stenting (pre-dilatation with an oversized balloon). Shortly after, they also demonstrated that stents with higher initial activity levels of $12-21 \mu \mathrm{Ci}$ (54 lesions) reduced intrastent neointimal hyperplasia compared with stents of 3-12 $\mu \mathrm{Ci}$ (42 lesions) [106]. However, they did not eliminate edge restenosis (38\% for the lower activity stents were reduced just to $30 \%$ for the higher activity stents). Since they used a non-aggressive stent implantation strategy (pre-dilatation with a non-oversized balloon) in the second study, they also ruled out that the edge effect is attributable to the implantation procedure. Wardeh et al. implanted 31 stents in 26 patients [107]. They corroborated this deduction (also attributing the edge effect to low radiation levels) and concluded that the use of low activity radioactive stents is safe and feasible. Sianos et al. analyzed 175 human vessels (131 were eventually eligible) treated according to the beta-radiation in Europe (BRIE) study protocol [108]. They wanted to evaluate the impact of Geographical Miss (GM-a situation in which the radiation source does not fully cover the injured vessel segment) on edge restenosis after intracoronary beta-radiation therapy. The injured edges of the effective irradiated segment (EIRS) constituted the GM edges. Restenosis was defined as diameter stenosis $>50 \%$ at follow-up (6 months). They found GM affected $41.2 \%$ of the edges and significantly increased edge restenosis to $16.3 \%$ compared with $4.3 \%$ in non-GM edges (for both proximal and distal edges). GM associated with stent injury increased edge restenosis more than that associated with balloon injury (from 3.6\% with no GM to $18.75 \%$ compared with from 5.36 to $10.71 \%$, respectively). However, EIRS restenosis was similar between vessels with and without GM (24.3 and 21.6\%, respectively), thus indicating a dominant effect for radiation fall origins. Van der Giessen et al. investigated the edge effect in the coronary arteries of Yucatan micropigs [109]. They fabricated half radioactive and half non-radioactive stents ( $n=20$, with 10 regular stent controls). Their design introduced a mid-stent radioactive dose falloff zone next to a non-radioactive stent-artery transition at one side and a radioactive stent-artery transition at the other side. They demonstrated a significant mid-stent stenosis at 4 weeks followup. Two animals died suddenly because of coronary occlusion at this mid-zone at 8 and 10 weeks. At 12 weeks, there was a significant neointimal thickening at the mid-stent dose-falloff zone of the half-radioactive stents but not at the stent-toartery transitions at both extremities. No mid-stent response was observed in the 
non-radioactive stents. They concluded that the edge effect is associated with the combination of stent injury and radioactive dose falloff. Studies performed on both human subjects and animal models found a significant neointimal increase at low activity stents edges [110]. These findings seem clinically significant since low-dose-related neointimal hyperproliferation, when compared with conventional radiosurgery, does not appear to be associated with damage to the Tunica Adventitia or Vasa Vasorum, hyaline phenotypes, or any endothelial disfunction (degeneration traits). Desouky et al. recently reviewed the targeted and non-targeted effects of ionizing radiation [111]. They describe cell and tissue response to low-dose/dose rate ionizing radiation. Cells exposed to such radiation exhibit increased cellular communication and resistance to future irradiation. They conclude that exposure of human cells to low radiation induces molecular processes that are different from those induced by high dose radiation. Furthermore, the effects of ionizing radiation are not restricted to irradiated cells but also affect non-irradiated cells via mechanisms termed "Radiation Bystander Effects" and "Radioadaptive Response" [111]. This suggests that a low-dose/dose rate source can potentially affect a larger volume than that anticipated from direct energy absorbance evaluations based on our experience with high-energy treatments.

The edge effect has always been treated as an adverse phenomenon. However, in AVM treatment, the objective is precisely the opposite of most traditional vascular procedures-obliteration rather than revascularization. Here, we suggest that if exploited properly, this effect may prove highly beneficial. Let us explain its potential advantages:

- An implant can be introduced in a minimally invasive manner by an interventional neuroradiologist alongside embolization or standalone. The expertise required for these procedures overlaps considerably.

- Gradual closure of the blood vessels will not elicit rapid hemodynamic irregularities that are well known to induce adverse effects such as hemorrhage.

- A single implantation procedure yields short overall operative/hospitalization time with FT or staged embolization. No recurrent patient admittance also ensures adequate costs compared with staged interventional procedures (also considering hospitalization charges).

- The literature indicates that lumen occlusion in these settings requires months. This can possibly lead to a shorter overall treatment period compared with FT or staged embolization (that can take up to years) and thus reduce hemorrhage risks.

- Treatment time for SRS is typically up to $\sim 2 \mathrm{~h}$. For FT, let us multiply it by up to 5 which is a common session number-indicating $\sim 10 \mathrm{~h}$. Let us now assume that the continuous source is decaying over a period of just 3 months $\sim 3^{*} 30$ day $/$ month $24 \mathrm{~h} /$ day $=2160 \mathrm{~h}$. This indicates that a dose rate more than a 1000 times smaller compared with that which is used in SRS or more than 200 times smaller compared with FT could eventually provide the same overall absorbed energy. This is clearly not a sufficient medical proof since we know FT is less efficient than radiosurgery in obliteration rates and we certainly have no evidence of the effectiveness of such a source type and radiation levels in the AVM context. However, it does clearly indicate the potential for doserate reduction. Many AREs that originally motivated FT could be inherently circumvented when mild constant radiation is employed instead of short-lived 


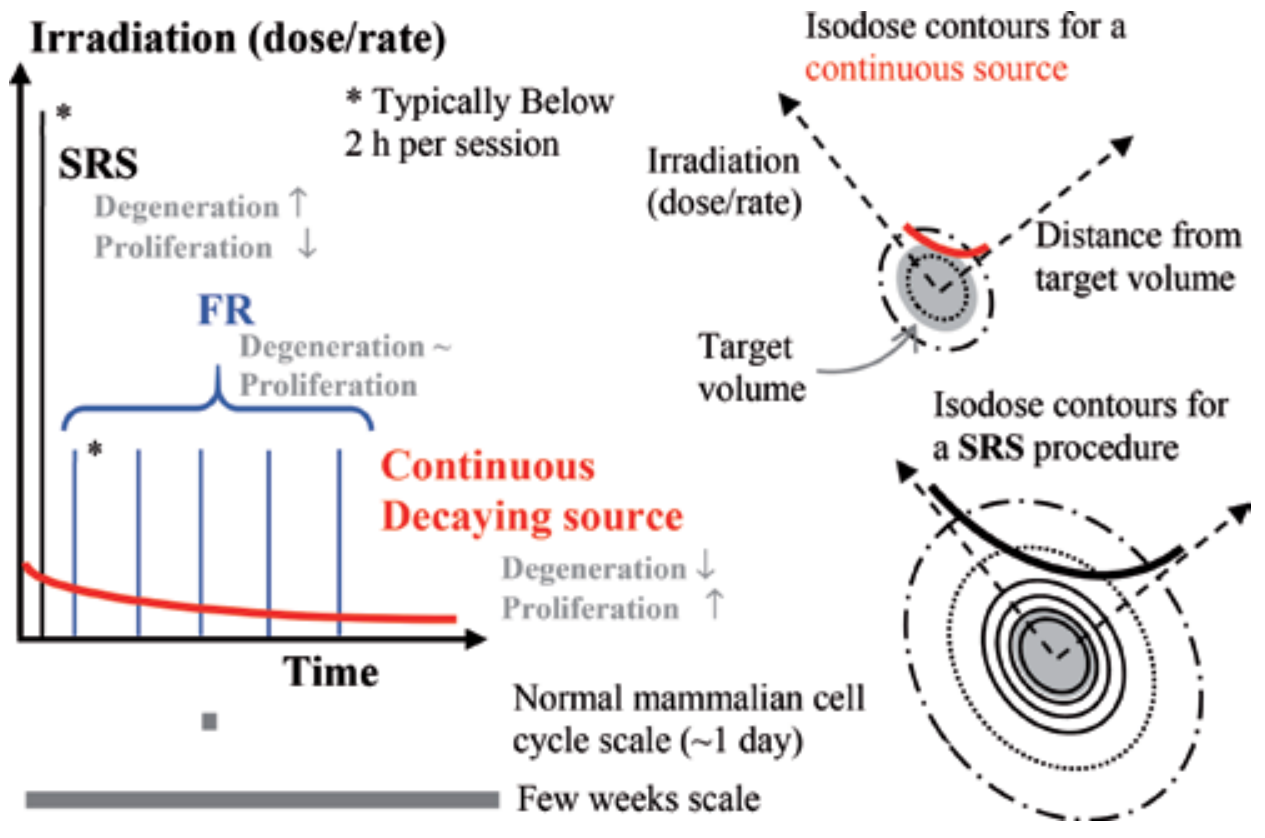

Figure 8.

Illustration of the conceptual differences between the presumed radiation traits required from a continuous source and those required from the current two predominant treatment modalities - radiosurgery and FT. Left: radiation dose/rate kinetics. Right: isodose map (the same dashing indicates equal radiation levels). The continuous source is constantly effective, thus necessitating significantly reduced doses/rates and facilitating a much better contained radiation distribution field, reduced affected brain volume, and potentially reduced AREs.

high-intensity energy doses/rates. Mild radiation also ensures a much better contained isodose distribution compared with the target volume and thus affected brain volume (Figure 8).

- A mammalian cell cycle typically lasts around a day ( $24 \mathrm{~h})$. A treatment lasting up to $2 \mathrm{~h}$ will not overlap with each of the cell cycle stages. However, a continuous source will expose the lesion cells to radiation during each of their cell cycle stages. We know from cancer treatment experience that this can prove highly beneficial. No data is available to indicate this also applies in the case of AVM treatment. However, this is certainly a point to consider.

- There is a mature and experienced medical device/implant industry that can reasonably rapidly adjust to develop and fine-tune such implants to AVM clinical requirements.

- Device/implant procedures are relatively easily attuned to qualify for reimbursement regulations, so insurance companies and medical centers can adopt the treatment modality.

- In contrast with embolic agents, a radiation source does not have to occupy each and every AVM blood vessel because it inherently supports a collateral effect. This leaves a very wide and flexible working range for the physicians regarding the final implantation position.

To conclude, if proven feasible, low-radiation implants could add several unique benefits to AVM treatment and we advocate studying their use. 


\section{Author details}

Nitzan Hirsh ${ }^{1 \dagger}$, Amir Arthur ${ }^{1 \dagger}$ and Saar Golan ${ }^{2 *}$

1 Luseed Vascular, Tel-Aviv, Israel

2 Chemical Engineering Department, Ariel University, Ariel, Israel

*Address all correspondence to: saarg@ariel.ac.il; thegolanfamily@gmail.com $\uparrow$ Equal contributors.

\section{IntechOpen}

(C) 2019 The Author(s). Licensee IntechOpen. This chapter is distributed under the terms of the Creative Commons Attribution License (http://creativecommons.org/licenses/ by/3.0), which permits unrestricted use, distribution, and reproduction in any medium, provided the original work is properly cited. (cc) BY 


\section{References}

[1] Ding D, Starke RM, Kano H, et al. Stereotactic radiosurgery for ARUBA (arandomized trial of unruptured brain arteriovenous malformations)eligible Spetzler-Martin grade I and II arteriovenous malformations: A multicenter study. World Neurosurgery. 2017;102:2-5

[2] Kim EJ, Vermeulen S, Li FJ, et al. A review of cerebral arteriovenous malformations and treatment with stereotactic radiosurgery. Translational Cancer Research. 2014;3:399-410

[3] Miller CE, Quayyum Z, McNamee P, et al. Economic burden of intracranial vascular malformations in adults prospective population-based study. Stroke. 2009;40:1973-1979

[4] Lv X, Wu Z, Jiang C, et al. Complication risk of endovascular embolization for cerebral arteriovenous malformation. European Journal of Radiology. 2011;80:776-779

[5] Yamamoto M, Kawabe T, Barfod BE. Long-term side effects of radiosurgery for arteriovenous malformations. Progress in Neurological Surgery. 2013;27:97-106

[6] Mohr JP, Parides MK, Stapf C, et al. Medical management with or without interventional therapy for unruptured brain arteriovenous malformations (ARUBA): A multicentre, nonblinded, randomised trial. The Lancet. 2014;383:614-621

[7] Al-Shahi R, Warlow C. A systematic review of the frequency and prognosis of arteriovenous malformations of the brain in adults. Brain. 2001;124(10):1900-1926

[8] Ajiboye N, Chalouhi N, Starke RM, et al. Cerebral arteriovenous malformations: evaluation and management. The Scientific World Journal. Hindawi Publishing Corporation. 2014: 6 p. Article ID 649036. DOI: $10.1155 / 2014 / 649036$

[9] Lawton MT, Rutledge WC, Helen K, et al. Brain arteriovenous malformations. Nature Reviews Disease Primers. 2015;1:1-20

[10] Collin A, Labeyrie MA, Lenck $S$, et al. Long term follow-up of endovascular management of spinal cord arteriovenous malformations with emphasis on particle embolization. Journal of NeuroInterventional Surgery. 2018. Available from: https://jnis.bmj. com/content/10/12/1183

[11] Chiang KH, Wang AH, Chang ET. Pulmonary arteriovenous malformation. American Journal of Respiratory and Critical Care Medicine. 2011;184:618

[12] Brown C, Subramanian V, Mel Wilcox C, Peter S. Somatostatin analogues in the treatment of recurrent bleeding from gastrointestinal vascular malformations: An overview and systematic review of prospective observational studies. Digestive Diseases and Sciences. 2010;55:2129-2134

[13] Fakhri A, Fishman EK, Mitchell SE, et al. The role of CT in the management of pelvic arteriovenous malformations. Cardiovascular and Interventional Radiology. 1987;10:96-99

[14] Gunterberg B. Renal arteriovenous malformation. Acta Radiologica. 1968;7:425-430

[15] Hashimoto M, Tate E, Nishii T, et al. Angiography of hepatic vascular malformations associated with hereditary hemorrhagic telangiectasia. CardioVascular and Interventional Radiology. 2003;26:177-180 
[16] Cho SK, Do YS, Shin SW, et al. Arteriovenous malformations of the body and extremities: Analysis of therapeutic outcomes and approaches according to a modified angiographic classification. Journal of Endovascular Therapy: An Official Journal of the International Society of Endovascular Specialists. 2006;13:527-538

[17] Hofmeister C, Stapf C, Hartmann A, et al. Demographic, morphological, and clinical characteristics of 1289 patients with brain arteriovenous malformation. Stroke. 2000;31:1307-1310

[18] Nagaraja S, Capener D, Coley SC, et al. Brain arteriovenous malformations: Measurement of nidal volume using a combination of static and dynamic magnetic resonance angiography techniques. Neuroradiology. 2005;47:387-392

[19] Ruíz-Sandoval JL, Cantú C, Barinagarrementeria $\mathrm{F}$. Intracerebral hemorrhage in young people. Stroke. 1999;30(3):537-541

[20] Spetzler RF, Hargraves RW, McCormick PW, et al. Relationship of perfusion pressure and size to risk of hemorrhage from arteriovenous malformations. Journal of Neurosurgery. 1992;76:918-923

[21] Markl M, Wu C, Hurley MC, et al. Cerebral arteriovenous malformation: Complex 3D hemodynamics and 3D blood flow alterations during staged embolization. Journal of Magnetic Resonance Imaging. 2013;38:946-950

[22] Chang W, Loecher MW, Wu Y, et al. Hemodynamic changes in patients with arteriovenous malformations assessed using high-resolution 3D radial phase-contrast MR angiography. American Journal of Neuroradiology. 2012;33:1565-1572

[23] Beneš V, Bradác O. Brain Arteriovenous Malformations-
Pathogenesis, Epidemiology, Diagnosis, Treatment and Outcome. Switzerland: Springer International Publishing AG; 2017

[24] Leblanc GG, Golanov E, Awad IA, Young WL. Biology of vascular malformations of the brain. Stroke. 2009;40(12):e694-e702

[25] Morris Z, Whiteley WN, Longstreth WT, et al. Incidental findings on brain magnetic resonance imaging: Systematic review and metaanalysis. British Medical Journal. 2009;339:b3016-b3016

[26] Stapf C, Overbey JR, Mohr JP, Moskowitz AJ, Vicaut E, Parides MK. New york: Université Paris Diderot. Available from: https://professional. heart.org/idc/groups/ahamah-public/@ wcm/@sop/@scon/documents/ downloadable/ucm_481657.pdf

[27] Motebejane MS, Royston D, Kabera G, et al. Demographic and angioarchitectural features associated with seizures presentation in patients with brain arteriovenous malformations in Durban, KwaZulu-Natal, South Africa. Interdisciplinary Neurosurgery: Advanced Techniques and Case Management. 2018;11:14-18

[28] Willinsky RA, Lasjaunias P, TerbruggeK, Burrows P. Multiplecerebral arteriovenous malformations (AVMs) Review of our experience from 203 patients with cerebral vascular lesions. Neuroradiology. 1990;32:207-210

[29] The Arteriovenous Malformation Study Group. Arteriovenous malformations of the brain in adults. The New England Journal of Medicine. 1999;340(23):1812-1818

[30] Stapf C, Mast H, Sciacca RR, et al. The New York Islands AVM study: Design, study Progress, and Initial Results. Stroke. 2003;34:e29-e33 
[31] Da Costa L, Wallace MC, Ter Brugge KG, O'Kelly C, Willinsky RA, Tymianski M. The natural history and predictive features of hemorrhage from brain arteriovenous malformations. Stroke. 2009;40:100-105

[32] Hartmann A, Mast H, Mohr JP, et al. Morbidity of intracranial hemorrhage in patients with cerebral arteriovenous malformation. Stroke. 1998;29(5):931-934

[33] Keedy A. An overview of intracranial aneurysms. McGill Journal of Medicine. 2006;9:141-146

[34] Arteriovenous MalformationsAANS Summary. Available from: https://www.aans. org/Patients/NeurosurgicalConditions-and-Treatments/ Arteriovenous-Malformations

[35] Kunc Z. The possibility of surgical treatment of arteriovenous malformations in anatomically important cortical regions of the brain. Acta Neurochirurgica. 1965;13:361-379

[36] Spetzler RF, Martin NA. A proposed grading system for arteriovenous malformations. Journal of Neurosurgery. 1986;65:476-483

[37] Conger A, Kulwin C, Lawton MT, et al. Endovascular and microsurgical treatment of cerebral arteriovenous malformations: Current recommendations. Surgical Neurology International. 2015;6:39

[38] Geibprasert S, Pongpech S, Jiarakongmun $\mathrm{P}$, et al. Radiologic assessment of brain arteriovenous malformations: What clinicians need to know. Radiographics. 2010;30:483-501

[39] Atlas SW. Magnetic Resonance Imaging of the Brain and Spine. 4th ed. Philadelphia: Lippincott Williams \& Wilkins; 2009
[40] Levin DC, Schapiro RM, Boxt LM, Dunham L, Harrington DP, Ergun DL. Digital subtraction angiography: Principles and pitfalls of image improvement techniques. American Journal of Roentgenology. 1984;143(3): 447-454

[41] Mossa-Basha M, Chen J, Gandhi D. Imaging of cerebral arteriovenous malformations and dural arteriovenous fistulas. Neurosurgery Clinics of North America. 2012;23:27-42

[42] Koenigsberg RA. Brain imaging in arteriovenous malformation. Medscape. 2017. Available from: https://emedicine. medscape.com/article/337220-overview

[43] Ellis JA, Lavine SD. Role of embolization for cerebral arteriovenous malformations. Methodist DeBakey Cardiovascular Journal. 2014;10(4):234-239

[44] Soltanolkotabi M, Schoeneman SE, Alden TD, et al. Onyx embolization of intracranial arteriovenous malformations in pediatric patients. JNS Pediatrics. 2013;11(4):431-437

[45] Derdeyn CP, Zipfel GJ, Albuquerque FC, et al. Management of brain arteriovenous malformations: A scientific statement for healthcare professionals from the American Heart Association/American Stroke Association. Stroke. 2017;48:e200-e224

[46] Forsting M, Wanke I. Intracranial Vascular Malformations and Aneurysms. Germany: Springer; 2008

[47] Jafar JJ, Awad IA, Rosenwasser RH, eds. Vascular Malformations of the Central Nervous System. Philadelphia: Lippincott Williams \& Wilkins; 1999

[48] Gupta V, Chugh M, Walia BS, Vaishya S, Jha AN. Use of CT angiography for anatomic localization of arteriovenous malformation nidal 
components. American Journal of

Neuroradiology. 2008;29:1837-1840

[49] Smith HJ, Strother CM,

Kikuchi Y, et al. MR imaging in

the management of supratentorial

intracranial AVMs. American

Journal of Roentgenology.

1988;150(5):1143-1153

[50] Lee BC, Herzberg L,

Zimmerman RD, Deck MD. MR imaging of cerebral vascular malformations.

American Journal of Neuroradiology.

$1985 ; 6: 863-870$

[51] Caramia F, Francia A,

Mainero C, et al. Neurophysiological

and functional MRI evidence of

reorganization of cortical motor areas

in cerebral arteriovenous malformation.

Magnetic Resonance Imaging.

2009;27:1360-1369

[52] Lee KE, Choi CG, Choi JW, et al. Detection of residual brain arteriovenous malformations after radiosurgery: Diagnostic accuracy of contrast-enhanced three-dimensional time of flight MR angiography at 3.0 tesla. Korean Journal of Radiology. 2009;10:333-339

[53] Wu C, Ansari SA, Honarmand AR, et al. Evaluation of 4D vascular flow and tissue perfusion in cerebral arteriovenous malformations: Influence of Spetzler-Martin grade, clinical presentation, and AVM risk factors. American Journal of Neuroradiology. 2015;36:1142-1149

[54] Alexander AL, Lee JE, Lazar M, et al. Diffusion tensor imaging of the brain. Neurotherapeutics.

2007;4:316-329

[55] Bendok BR, El Tecle NE, El Ahmadieh TY, et al. Advances and innovations in brain arteriovenous malformation surgery. Neurosurgery. 2014;74:S60-S73
[56] Steinmeier R, Schramm J, Müller $\mathrm{H}-\mathrm{G}$, et al. Evaluation of prognostic factors in cerebral arteriovenous malformations. Neurosurgery. 1989;24(2):193-200

[57] de Oliveira E, Tedeschi H, Raso J. Comprehensive management of arteriovenous malformations. Neurological Research. 1998; 20(8):673-683

[58] Lawton MT, Solomon RA, Raso J, et al. Spetzler-Martin grade III arteriovenous malformations: Surgical results and a modification of the grading scale. Neurosurgery. 2003;52:740-749

[59] Neidert MC, Lawton MT, Marius M, et al. The AVICH score: A novel grading system to predict clinical outcome in arteriovenous malformation-related intracerebral hemorrhage. World Neurosurgery. 2016;92:292-297

[60] Lawton MT, Kim H, McCulloch CE, et al. A supplementary grading scale for selecting patients with brain arteriovenous malformations for surgery. Neurosurgery. 2010;66(4):702-713

[61] Schwartz M, Sixel K, Young C, et al. Prediction of obliteration of arteriovenous malformations after radiosurgery: The obliteration prediction index. Canadian Journal of Neurological Sciences. 1997;24:106-109

[62] Pollock BE, Flickinger JC. A proposed radiosurgery-based grading system for arteriovenous malformations. Journal of Neurosurgery.

2002;96(1):79-85

[63] Pollock BE, Storlie CB, Link MJ, et al. Comparative analysis of arteriovenous malformation grading scales in predicting outcomes after stereotactic radiosurgery. Journal of Neurosurgery. 2016:1-7 
[64] Feliciano CE, de León-Berra R, Hernández-Gaitán MS, et al. A proposal for a new arteriovenous malformation grading scale for neuroendovascular procedures and literature review. PRHSJ. 2010;29(2):117-120

[65] Frizzel RT, Fisher WS. Cure, morbidity, and mortality associated with embolization of brain arteriovenous malformations: Areview of 1246 patients in 32 series over a 35-year period. Neurosurgery. 1995;37(6):1031-1040

[66] Reig AS, Rajaram R, Simon S, et al. Complete angiographic obliteration of intracranial AVMs with endovascular embolization: Incomplete embolic nidal opacification is associated with AVM recurrence. Journal of Neurosurgery. 2010;2:202-207

[67] Yang W, Porras JL, Xu R, et al. Comparison of hemorrhagic risk in intracranial arteriovenous malformations between conservative management and embolization as the single treatment modality. Neurosurgery. 2018;481-490(2018):82

[68] Bruno CA Jr, Meyers PM. Endovascular management of arteriovenous malformations of the brain. Interventional Neurology. 2013;1(3-4):109-123

[69] Vaidya S, Tozer KR, Chen J. An overview of embolic agents. Seminars in Interventional Radiology. 2008;25(3):204-215

[70] Panagiotopoulos V, Gizewski E, Asgari S, et al. Embolization of intracranial arteriovenous malformations with ethylenevinyl alcohol copolymer (Onyx). American Journal of Neuroradiology. 2009;30:99-106

[71] Tawil I, Carlson AP, Taylor CL. Acute respiratory distress syndrome after Onyx embolization of arteriovenous malformation. Critical Care Research and Practice. 2011;2011:1-5

[72] Vollherbst DF, Otto R, von Deimling A, et al. Evaluation of a novel liquid embolic agent (precipitating hydrophobic injectable liquid (PHIL)) in an animal endovascular embolization model. Journal of Neurosurgery. 2018;10:268-274

[73] Vollherbst DF, Sommer CM, Ulfert C, et al. Liquid embolic agents for endovascular embolization: Evaluation of an established (Onyx) and a novel (PHIL) embolic agent in an in vitro AVM model. American Journal of Neuroradiology. 2017;38(7):1377-1382

[74] Varadharajan S, Ramalingaiah A, Saini J, et al. Precipitating hydrophobic injectable liquid embolization of intracranial vascular shunts: Initial experience and technical note. Journal of Neurosurgery. 2017;129(5):1217-1222

[75] Jordan JA, Llibre JC, Vázquez F, et al. Predictors of hemorrhagic complications from endovascular treatment of cerebral arteriovenous malformations. Interventional Neuroradiology. 2014;20:74-82

[76] Hartmann A, Pile-Spellman J, Stapf C, et al. Risk of endovascular treatment of brain arteriovenous malformations. Stroke. 2002;33:1816-1820

[77] Haw CS, terBrugge K, Willinsky R, et al. Complications of embolization of arteriovenous malformations of the brain. Journal of Neurosurgery. 2006;104(2):226-232

[78] Jayaraman MV, Marcellus ML, Hamilton S, et al. Neurologic complications of arteriovenous malformation embolization using liquid 
embolic agents. American Journal of Neuroradiology. 2008;29(2):242-246

[79] Badra EV, Ermiş E, Mordasini P, et al. Radiosurgery and radiotherapy for arteriovenous malformations: Outcome predictors and review of the literature. Journal of Neurosurgery. 2018:490-504

[80] Greitz T, Lax I, Bergström M, et al. Stereotactic radiation therapy of intracranial lesions methodologic aspects. Acta Radiologica: Oncology. 1986;25(2):81-89

[81] Lunsford LD, Niranjan A, Kondziolka D, et al. Arteriovenous malformation radiosurgery: A twenty year perspective. Clinical Neurosurgery. 2008;55:108-119

[82] Powers BE, Thames HD, Gillette EL. Long-term adverse effects of radiation inhibition of restenosis: Radiation injury to the aorta and branch arteries in a canine model. International Journal of Radiation Oncology Biology Physics. 1999;45:753-759

[83] Szeifert GT, Levivier M, Lorenzoni J, et al. Morphological observations in brain arteriovenous malformations after gamma knife radiosurgery. Progress in Neurological Surgery. 2013;27:119-129

[84] Major O, Szeifert GT, Radatz MWR, et al. Experimental stereotactic gamma knife radiosurgery. Vascular contractility studies of the rat middle cerebral artery after chronic survival. Neurological Research. 2002;24:191-198

[85] Deruty R, Pelissou-guyotat I, Morel C, et al. Reflections on the management of cerebral arteriovenous malformations. Surgical Neurology. 1998;50:245-256

[86] Yen CP, Matsumoto JA, Wintermark M, et al. Radiationinduced imaging changes following gamma knife surgery for cerebral arteriovenous malformations. Journal of Neurosurgery. 2013;118:63-73

[87] Pollock BE, Link MJ, Branda ME, et al. Incidence and management of late adverse radiation effects after arteriovenous malformation radiosurgery. Neurosurgery. 2017;81(6):928-934

[88] Pollock BE, Link MJ, Stafford SL, et al. The risk of radiation-induced tumors or malignant transformation after single-fraction intracranial radiosurgery: Results based on a 25-year experience. International Journal of Radiation Oncology, Biology, Physics. 2017;97(5):919-923

[89] Unkelbach J, Bussière MR, Chapman $\mathrm{PH}$, et al. Spatiotemporal fractionation schemes for irradiating large cerebral arteriovenous malformations. International Journal of Radiation Oncology, Biology, Physics. 2016;95(3):1067-1074

[90] Kim H, Abla AA, Nelson J, et al. Validation of the supplemented Spetzler-Martin grading system for brain arteriovenous malformations in a multicenter cohort of 1009 surgical patients. Neurosurgery. 2015;76:25-31

[91] Schramm J, Schaller K, Esche J, et al. Microsurgery for cerebral arteriovenous malformations: Subgroup outcomes in a consecutive series of 288 cases. Journal of Neurosurgery. 2017;126(4):1056-1063

[92] Ren Q, He M, Zeng Y, et al. Microsurgery for intracranial arteriovenous malformation: Long-term outcomes in 445 patients. PLoS One. 2017;12(3):e0174325

[93] Wong J, Slomovic A, Ibrahim G, et al. Microsurgery for ARUBA trial (arandomized trial of unruptured brain arteriovenous malformation)eligible unruptured brain arteriovenous malformations. Stroke. 2017;48:136-144 
[94] Gross BA, Storey A, Orbach DB, et al. Microsurgical treatment of arteriovenous malformations in pediatric patients: The Boston Children's Hospital experience. JNS Pediatrics. 2015;15:71-77

[95] Abla AA, Rutledge WC, Seymour ZA, et al. A treatment paradigm for high-grade brain arteriovenous malformations: Volume-staged radiosurgical downgrading followed by microsurgical resection. Journal of Neurosurgery. 2015;122:419-432

[96] Aboukaïs R, Vinchon M, Quidet M, et al. Reappearance of arteriovenous malformations after complete resection of ruptured arteriovenous malformations: True recurrence or falsenegative early postoperative imaging result? Journal of Neurosurgery. 2017;126(4):1088-1093

[97] Rad NR, McRobb LS, Zhao Z, et al. Phosphatidylserine translocation after radiosurgery in an animal model of arteriovenous malformation. Radiation Research. 2017;187(6):701-707

[98] Kockro RA, Reisch R, Serra L, et al. Image-guided neurosurgery with 3-dimensional multimodal imaging data on a stereoscopic monitor. Neurosurgery. 2013;72:A78-A88

[99] Spetzler RF, Sanai N. The quiet revolution: Retractorless surgery for complex vascular and skull base lesions. Journal of Neurosurgery. 2012;116:291-300

[100] Ross J, Salman RA. Interventions for treating brain arteriovenous malformations in adults. Cochrane Database of Systematic Reviews. 2010;(7). Art. No.: CD003436. Available from: https://www.cochranelibrary. com/cdsr/doi/10.1002/14651858. CD003436.pub3/full\#

[101] Magro E, Gentric J, Darsaut TE, et al. Responses to ARUBA: A systematic review and critical analysis for the design of future arteriovenous malformation trials. Journal of Neurosurgery. 2017;126(2):486-494

[102] Cenzato M, Delitala A, Delfini R, et al. Position statement from the Italian Society of Neurosurgery on the ARUBA study. Journal of Neurosurgical Sciences. 2016;60(1):126-130

[103] Hong CS, Peterson EC, Ding D, et al. Intervention for a randomized trial of unruptured brain arteriovenous malformations (ARUBA)_Eligible patients: An evidence-based review. Clinical Neurology and Neurosurgery. 2016;150:133-138

[104] Cenzato M, Boccardi E, Beghi E, et al. European consensus conference on unruptured brain AVMs treatment (supported by EANS, ESMINT, EGKS, and SINCH). Acta Neurochirurgica. 2017;159:1059-1064

[105] Albiero R, Adamian M, Kobayashi N, et al. Short- and intermediate-term results of 32P radioactive $\beta$-emitting stent implantation in patients with coronary artery disease. Circulation. 2000;101:18-26

[106] Albiero R, Nishida T, Adamian M, et al. Edge restenosis after implantation of high activity $32 \mathrm{P}$ radioactive $\beta$-emitting stents. Circulation. 2000;101:2454-2457

[107] Wardeh AJ, Kay IP, Sabaté M, et al. $\beta$-Particle-emitting radioactive stent implantation asafety and feasibility study. Circulation. 1999;100:1684-1689

[108] Sianos G, Kay IP, Costa MA, et al. Geographical miss during catheter-based intracoronary betaradiation: Incidence and implications in the BRIE study. Journal of the American College of Cardiology. 2001;38(2):415-420 
[109] van der Giessen WJ, Regar E, Harteveld MS, et al. "Edge effect" of $32 \mathrm{P}$ radioactive stents is caused by the combination of chronic stent injury and radioactive dose falloff. Circulation. 2001;104:2236-2241

[110] Hansen A, Hehrlein C, Hardt S, et al. Is the "candy-wrapper" effect of $32 \mathrm{P}$ radioactive $\beta$-emitting stents due to remodeling or neointimal hyperplasia? Insights from intravascular ultrasound. Catheterization and Cardiovascular Interventions. 2001;54:41-48

[111] Desouky O, Ding N, Zhou G. Targeted and non-targeted effects of ionizing radiation. Journal of Radiation Research and Applied Sciences.

2015;8(2):247-254 

Section 3

\section{Blister Aneurysms}





\title{
Chapter 6
}

\section{Blister Aneurysms}

\author{
Themistoklis I. Papasilekas, Konstantinos M. Themistoklis, \\ Stefanos I. Korfias and Damianos E. Sakas
}

\begin{abstract}
Blister aneurysms are rare carotid lesions characterized by a thornlike appearance in combination with prominent fragility. Comprising less than $2 \%$ of all intracranial aneurysms, they are considered to be either dissecting or false aneurysms. Etiogenesis remains poorly understood, though atherosclerosis seems to be playing a prominent role. Although many approaches have been tried throughout the years, treatment of blister lesions remains debatable. Both surgical and endovascular modalities can be used, with every technique having though its own limitations and pitfalls. In this context, when confronted with such a lesion, physicians should consider all available alternatives in order to maximize the chances of a good outcome.
\end{abstract}

Keywords: blister aneurysms, blister-like aneurysms, carotid aneurysms

\section{Introduction}

Blister aneurysms are a rare but well-recognized form of cerebral vascular lesions. Comprising less than $2 \%$ of all intracranial aneurysms, they are typically found on the dorsal or dorsomedial wall of the internal carotid artery (ICA). With a characteristic thornlike appearance on angiography, blister aneurysms' most prominent feature is the fragility of their wall. This explains for their aggressive clinical course and grave prognosis.

Although most authors agree that blister aneurysms are either dissecting or false lesions, their exact nature as well as their optimal management remains unknown. Traditionally, surgery has been advocated as the first-line treatment. Primary clipping, wrapping, wrap-clipping or even carotid artery sacrifice (with or without a bypass) have all been tried. However, results have always been far from satisfying, often making neurosurgeons reluctant to operate on such cases.

During the past few years, clinicians' interest in blister aneurysm has been renewed with the introduction of endovascular modalities in everyday practice. Among all the different available approaches, flow diversion seems lately to be gaining ground, showing promising results. Of course, until consensus has been reached, blister aneurysms are still to be treated on a case-by-case basis.

\section{General features and clinical presentation}

Blister aneurysms are a distinct form of cerebral vascular lesions with often mixed characteristics and uncertain pathogenesis. They comprise less than $2 \%$ of all intracranial aneurysms [1]. Originally described by Sundt and Murphey more than 
40 years ago [2], the typical configuration of blister aneurysms consists of a shallow and broad-based or even semifusiform focal protrusion located on the internal carotid artery [3]. Arising from the anteromedial carotid wall in up to $65 \%$ of cases [4], these lesions seem to be unrelated to major arterial junctions, although very fine branches such as those supplying the optic nerve may sometimes be involved [5]. Rarely, blister aneurysms can be found on the anterior communicating artery or even the basilar trunk [6, 7], while in a recent case report, the posterior inferior cerebellar artery has also been implicated as another potential site of origin for such a lesion [8].

A prominent characteristic of blister aneurysms is the marked weakness of their wall, a feature that not only reflects the unique pathology of these lesions but also predetermines their high rupture risk, aggressive clinical course, and tendency for rapid growth and progression. In the most common scenario, a blister aneurysm will be diagnosed after an episode of subarachnoid hemorrhage (WFNS grade $>3$ in $68 \%$ of cases) [9]. Being initially small and sessile, it will substantially enlarge within days of presentation, reaching finally a shape much similar to that of its saccular counterparts [4]. Commonly, the end result is a rerupture with potentially catastrophic consequences for the patient.

On a side note, and regarding terminology, blister aneurysms were originally known as dorsal, anterior or superior carotid wall lesions, a nomenclature though that soon became obsolete failing to recognize the presence of such anomalies on the medial, posteromedial or lateral surface of the internal carotid artery [10]. Additionally, intracranial vessels have never-by tradition-been designated as dorsal or ventral, adding another reason to abandon at least the first of these denominations [11]. An alternative, broader term used mainly by Japanese authors was carotid trunk aneurysms [4]. Still, confusion remained since bleb-like but essentially stable carotid lesions have been encountered during surgery, an observation that clearly indicates that not all such morphological entities fulfill the requirements to be considered as true blister aneurysms, and as a matter of fact, some of them may even be the precursors of typical berry lesions $[12,13]$. Whatever the case, the term blister (or blister-like) aneurysms seems to be by now the dominant one within the relevant literature and as such will be used throughout the present manuscript.

\section{Epidemiology and demographics}

Blister aneurysms are rare lesions comprising less than $2 \%$ of all intracranial aneurysms [1] and 0.9-6.6\% of internal carotid artery lesions [14]. In the three largest series published to date, Yaşargil et al. reported 3 blister aneurysms in a total of 319 carotid lesions [15], Nakagawa et al. reported 8 cases in a series of 460 surgical patients [3] and Meling et al. reported 14 lesions in a total of 912 aneurysms [16].

Having a slight female preponderance and occurring more frequently on the right side, blister aneurysms tend to affect patients at a rather younger age than their saccular counterparts $[17,18]$. In a series of six patients, Abe et al. found a mean presentation age of 56 years [11], while Park et al. calculated this in their own cases at 35.4 years [19]. Risk factors include arterial hypertension [6] and atherosclerosis [16].

\section{Pathological considerations}

Due to the rarity of blister aneurysms, our understanding of the relevant pathology is only limited. For most authors these lesions are of a dissecting nature [20], and, as such, they are considered to be the consequence of a tear in the affected artery's inner wall followed by intramural hemorrhage [21, 22]. The resultant protrusion is 
covered by only the adventitial layer. At this point, it should be noted that the adventitia of intracranial arteries is well known to be thicker over bifurcation sites, partially compensating for the underlying medial defect. Arising exclusively at nonbranching arterial segments, blister aneurysms lack this relatively rigid coverage and are thus much more fragile than their saccular counterparts [23]. This difference between the two accounts for the prominently aggressive clinical course of blister lesions (i.e. increased risk of bleeding and tendency for rapid subsequent growth and rerupture). In their series of 40 blister aneurysms, Ogawa et al. found signs of dissection (double lumen, arterial narrowing or dilation, etc.) in 10 cases [4], while Satoh et al. reported a similar association in as many as 16 out of their 18 in total patients [13].

An alternative hypothesis on the pathology of blister aneurysms is that they are in fact false lesions, essentially representing a focal arterial wall defect [8]. In this direction, Abe et al. suggested a few years back that the characteristic bleb-like protrusion seen by surgeons in such cases is nothing more than an organized blood clot covering the diseased arterial site [11]. Removal of this clot during surgical preparation of the aneurysmal dome is highly likely to cause a rerupture [4]. The pseudoaneurysm theory was initially based on macroscopic intraoperative findings but was later confirmed in at least two cases reported by Charbel et al. [24]. According to these authors, pathological examination of the aneurysmal sacs, which were resected in both cases, in their entirety revealed only blood clot and no fibrous, elastic or smooth muscle tissue (Verhoeff-vanGieson staining plus immunostaining) [24].

The first-ever complete microscopic description of a blister aneurysm has been delivered by Ishikawa et al. in their, classic by now, publication in neurosurgery in 1997. To do this, the authors of the article applied an Elastica Masson stain on cross section specimens of the internal carotid artery of a patient who sustained a fatal rupture of such a lesion [25]. According to their report, the examined blood vessel was heavily atherosclerotic with prominent underlying thickening of the intima. Near the edge of the atheromatous plaque, both the internal elastic lamina and the tunica media were abruptly terminated, and the resultant gap (i.e. the aneurysm sac) was covered only with fibrinous tissue and adventitia. The latter was neither thickened nor rich in collagen as is usually seen in a saccular aneurysm. At the rupture point, the adventitia was lacerated and fragmented. Notably, and contrary to what it would be expected in a dissecting lesion, no inflammatory infiltration or dissection of the artery outside the actual aneurysm was observed.

In a more recent publication, Kim et al. reported a blister aneurysm with a typical clinical course whose dome was resected during surgery for histologic examination. Their conclusions, although interesting, seem to have only added to the confusion: immunohistochemical staining for smooth muscle actin proved to be positive, a finding compatible with an intact tunica media and thus a true lesion. Medial fibroblast proliferation and laminar thrombosis were also noted [26].

It is obvious that the literature on pathology of blister aneurysms has been, up until now, far from conclusive. Speculations stray widely with data supporting each theory being only limited. A possible explanation is that we are dealing with different stages of a rapidly evolving disease or, alternatively, that there is no single entity but a whole range of these. Our only certainty seems to be that morphological changes taking place in blister aneurysms are much more destructive than those seen in berry lesions.

\section{Etiogenesis}

Similar to their pathology, etiogenesis of blister aneurysms remains, despite our best efforts, unclear. Both Stehbens and Ohara have traditionally associated such 
lesions with atherosclerosis, a concept that has by now gained wide acceptance $[12,27]$. Atheromatous changes affecting the carotid artery lead to degeneration of the internal elastic lamina [28]. The latter is reported to be the major anatomic structure resisting the pressure of blood flow within a given vessel [29]. The end result is an overall weakening and subsequent laceration of the arterial wall (penetrating ulceration) [30]. Focal subadventitial dissection leads to the formulation of a blister aneurysm [14]. For reasons not yet fully understood, this whole process usually takes place at the periphery of an atherosclerotic plaque where its stiff degenerated wall borders that of a normal elastic vessel segment [25]. Further research is needed to explain carotid vulnerability at these locations.

Apart from atherosclerosis, another factor that seems to play an important role in the formation of blister aneurysms is hemodynamics. The anteromedial surface of the supraclinoid carotid segment, where most of blister lesions arise, is curved in such a way that blood flow directly impinges on the arterial wall $[4,11]$. In the most probable scenario, increased hemodynamic stress acts on an already diseased, sclerotic arterial segment, and it is the combination of these two that finally results in an aneurysm formation.

\section{Diagnosis}

Given that the vast majority of blister aneurysms presents with subarachnoid hemorrhage, the diagnostic evaluation of such a lesion usually starts with a brain CT scan. Hemorrhage is typically lateralized and mainly involves the carotid and suprasellar cisterns as well as the Sylvian fissure. Of note is that, when performed within 24 hours after ictus, CT scans detect subarachnoid blood with a sensitivity of up to $95 \%$, a figure though that quickly drops to less than $50 \%$ a week later [31]. This is attributed to the rapid decrease of the hemorrhage density due to dilution by cerebrospinal fluid [32].

The second step in the diagnostic triage of subarachnoid hemorrhage is, for most centers nowadays, a CT angiogram. Blister aneurysms appear, initially at least, as shallow, broad-based lesions, usually less than $2 \mathrm{~mm}$ and with a characteristic triangular or thornlike shape (Figure 1) [33,34]. Unfortunately, their small size in combination with their unusual location (i.e. nonbranching arterial sites) and close proximity to the skull base often makes detection of these lesions obscure [35]. Adding to the difficulty, the aneurysmal dome presumably collapses right after rupture, while its parent artery contracts as a reaction to the presence of subarachnoid blood. The end result can be a significant delay in diagnosis and initiation of treatment. Sensitivity of single slice CT angiography in the investigation of intracranial aneurysms smaller than $3 \mathrm{~mm}$ has been reported to be $25-64 \%$ [36]. Better results with improved image quality and spatial resolution have been achieved with the introduction of multidetector row technology [37]. Blister lesion diagnosis can be also greatly facilitated through the application of a meticulous technique (decreased section thickness, increased pitch, proper bolus timing and elimination of venous contamination) along with appropriate postprocessing of CTA scans (maximum intensity projections, multiplanar reconstructions and volume-rendered 3D images) [33]. Notably, most false-negative CT angiograms, when evaluated in retrospect, do reveal suspicious anomalies that could be well associated with a blister aneurysm. This observation underlines the significance in such cases of a high index of suspicion.

Despite advances in the field of CT angiography, conventional DSA with its excellent spatial resolution remains the gold standard for the detection of cerebral aneurysms, and, as such, it should be performed whenever initial investigations prove to be negative. The appearance of blister lesions on a DSA closely resembles that of their CTA counterparts, but luminar irregularities related to atherosclerosis of adjacent arterial segments may obscure the diagnosis (Figure 2) [35]. Multiple 


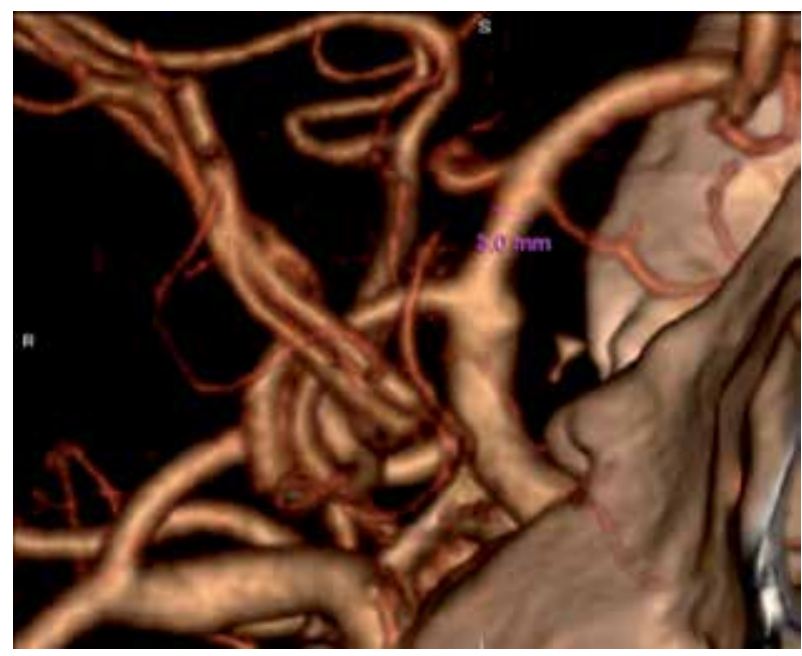

Figure 1.

Reconstructed CTA showing a blister-like aneurysm on the dorsal wall of the internal carotid artery (ICA).

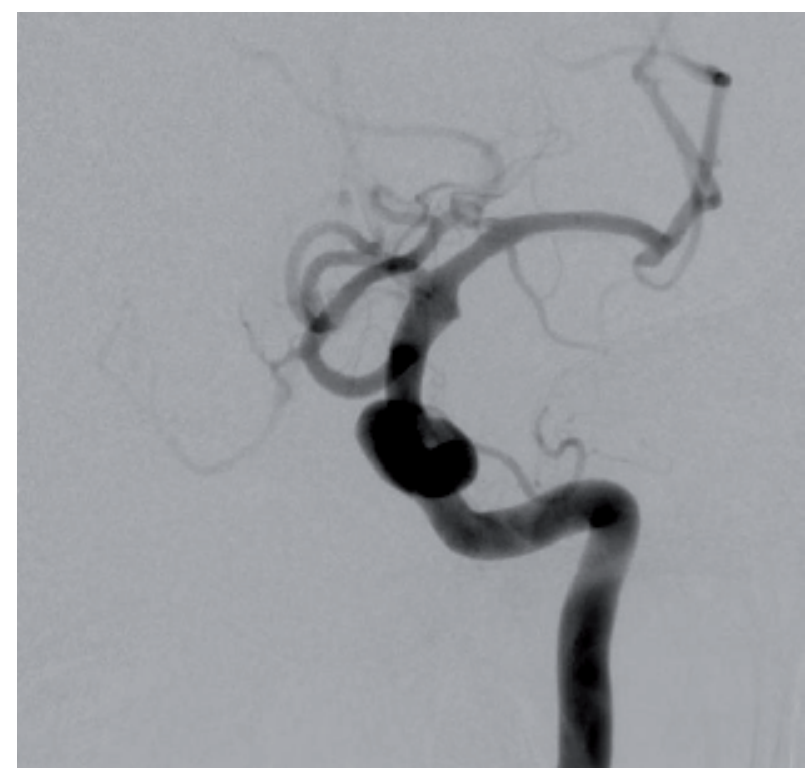

Figure 2.

Internal carotid injection, AP view. A characteristic thornlike blister aneurysm is noted opposite to the anterior choroidal artery origin. Large posterior communicating artery supplying the posterior circulation noted.

oblique views or even rotational 3D scans significantly increase the sensitivity of the method [38]. Signs of dissection have been reported in up to $89 \%$ of blister aneurysm cases and include a false lumen, an intimal flap, a filling defect or contrast pooling [39]. Should the presence of a blister aneurysm be suspected on the basis of DSA findings, the evaluation of collateral flow through the circle of Willis is always advisable in case an occlusion procedure is to be carried out $[18,33]$.

Cross-compression carotid injections may help demonstrate the anterior communicating artery, while patency, size and collateral potential of its posterior counterpart can be assessed through an Alcock test (vertebral injections with carotid artery compression). For a more detailed study, temporary balloon occlusion will be required [31]. 
Another important aspect of imaging in cases of blister aneurysms is that, on short-term follow-up angiography after initial presentation, these lesions usually show rapid growth to a saccular configuration [19]. Being at least partially related to lysis of an intra-aneurysmal clot [26], this progression is considered to be a good indicator of a blister lesion even though the only real way to authenticate such a diagnosis is through direct intraoperative inspection $[1,40]$.

\section{Treatment}

Management of blister aneurysms is associated with a high overall rate of mortality and morbidity [35]. The main causes for this include the small size and broad neck morphology along with the prominent fragility of such lesions, features that often lead to intraprocedural rupture when traditional surgical or endovascular techniques such as clipping or primary coiling are to be applied $[6,19]$. Additionally, and even if an initial intervention proves successful, subsequent regrowth requiring further treatment has been commonly reported [9]. Other factors contributing to the grim prognosis of blister aneurysms include a commonly grave clinical presentation as well as delays in an appropriate diagnosis.

Given the lack of universal consensus in blister aneurysm treatment, a wide variety of approaches and methods has been employed up to date and will be discussed below. These have included both reconstructive and deconstructive surgical and endovascular techniques, with different authors competing for best results in an ever-changing field. In any case, attention should be given to specific measures to prevent rerupture while awaiting final treatment (tight arterial blood pressure control, cautious cerebrospinal fluid drainage in patients with a ventricular drainage in place and selective use of aminocaproic acid) [41]. The latter must be instituted as soon as possible to secure the aneurysm and to allow aggressive management of subarachnoid hemorrhage-related complications such as vasospasm and hydrocephalus.

The alternative treatment modalities for blister aneurysms are:

a. Reconstructive techniques

- Surgery: primary clipping (including encircling clips), wrapping, clip-wrapping, wrap-clipping and direct suturing

- Endovascular therapy: primary coiling, stent-assisted coiling, telescopic stenting (stent-in-stent technique) and flow diverters

b.Deconstructive techniques

- Parent artery occlusion with surgical or endovascular means with or without bypass surgery

\subsection{Surgical treatment}

\subsubsection{Clipping procedures (primary clipping and wrap-clipping)}

\subsubsection{Technique}

Traditionally, surgical clipping has been the preferred mode of treatment for all forms of cerebral aneurysms, including blister lesions. In a typical case, the procedure starts with exposure of the cervical internal carotid artery to ensure 
proximal control in case of an intraprocedural rupture. This is usually achieved with an incision along the medial border of the ipsilateral sternocleidomastoid muscle [42]. Subsequently, a standard pterional craniotomy with generous sphenoid ridge drilling is carried out, and through this, the Sylvian fissure is opened widely. Gentle retraction of the frontal lobe provides access to the supraclinoid internal carotid artery which, in most cases, is found to be prominently sclerotic [43]. The aneurysm itself is usually seen protruding from the dorsomedial carotid wall. Careful preparation of the aneurysmal dome is crucial. As a matter of fact, should the frontal lobe be attached to it, most authors propose a subpial dissection in order to minimize direct manipulation of the lesion [44]. Special care is needed to avoid removing the platelet plug that typically covers the aneurysm as this may result in a large wall defect and uncontrollable bleeding [4].

Direct clipping of a blister aneurysm is performed under temporary trapping and in such a way that the blades of a usually angled or curved Sugita clip are parallel to the longitudinal axis of the carotid artery [19, 45]. This has been shown to lower the risk of intraprocedural rupture [17]. Given that the underlying pathological process seems to extend well beyond the limits of the aneurysm itself, the surgeon should try, when closing the clip, to include part of the "normal" arterial wall outside the lesion in order to avoid breakage of the transitional zone found in between them [4]. A valid alternative is to envelope the entire diseased arterial segment with a wrapping material such as gauze, cotton or Gore-Tex on top of which the clip can be applied to obliterate the lesion [46]. Wrap-clipping not only reinforces the carotid wall as a whole but also helps avoid slippage of the clip, a complication not uncommonly seen in blister aneurysms surgery [47]. One yet alternative is to wrap the lesion after the clip has been applied (clip-wrapping). Whatever the exact technique, induced hypotension [11], burst suppression with desflurane or thiopental, cooling of the patient or even transient flow arrest with adenosine can all prove useful adjuncts $[11,24,41,48]$.

\subsubsection{Results of clipping procedures, associated complications and rescue measures}

Primary clipping of blister lesions is known to carry an up to $30 \%$ risk of complications, both intraoperative and postoperative $[9,49]$. In the former group, aneurysm avulsion and internal carotid artery laceration seem to be our major concerns, while progression of the lesion often accompanied by rerupture seems to be the main danger during the postoperative period [50].

When confronted with a laceration of the carotid artery during surgery, the most commonly used rescue technique has traditionally been sacrifice of the vessel to control bleeding. As proven by numerous studies, this is associated with a high risk of cerebral ischemia, not only because collaterals may be inadequate in the first place but also due to hemorrhage-related vasospasm that often further aggravates the whole situation [16]. Other alternatives include direct suturing, placement of an encircling clip graft and reapplication of the originally placed clip to intentionally narrow the carotid artery and thus achieve hemostasis [4, 43]. In every case, surgeons must be always prepared for such an event, and large aspirators need to be available throughout the procedure. Initial hemorrhage control is usually achieved by applying direct pressure on the artery with oxidized cellulose and a small cottonoid. Brief periods of cardiac arrest with the aid of adenosine are also useful to improve visibility and help gain control of the situation [41].

Direct suturing of an arterial tear during surgery for a blister aneurysm is made possible because the edge of the lesion is relatively well outlined [51]. It entails removal of the original clip, trimming of the aneurysmal sac with microscissors (to prevent tissue buckling into the lumen) and repair of the arterial wall defect with 
$8 / 0$ nylon stitches [43]. Suturing in the acute stage is usually extremely difficult due to the deep and narrow surgical field. As a result, initial attempts at this required prolonged trapping of the diseased segment invariably led to cerebral ischemia [52, 53]. To avoid this, recent studies propose reformation of the arterial wall with only a few stitches rather than its complete restoration [43]. Subsequent circumferential wrapping of the artery or, alternatively, placement of an encircling clip graft should be enough to stop oozing and stabilize the end result [54].

Encircling clip grafts have been developed by Sundt almost 40 years ago and specifically to address the problem of repairing the wall defect associated with a blister aneurysm $[2,55]$. They can be used either as a primary modality (instead of the classic clip) or in cases of intraoperative rupture. Applied typically with the aid of a straight or right angle clip holder, Sundt clips provide a rigid sleeve and a soft woven fabric lining, allowing surgeons to essentially reconstruct the lacerated arterial wall [56]. Produced in various sizes, the appropriate clip for each case can be estimated on the basis of preoperative angiographic studies with a 3-4 mms diameter and a 3-5 mms length being the most widely used options [42]. Major disadvantages associated with these devices are that they cannot be used in cases of lesions close or opposite to carotid branches (i.e. posterior communicating and anterior choroidal arteries) and also that they come in certain fixed diameters that may or may not fit the vessel involved [48]. The latter limitation occasionally leads to postprocedural stenosis, a risk that surgeons must always be aware of and act accordingly [6]. Of note is a recent publication by Cho et al. who used a Sundt clip in combination with an endovascularly placed carotid stent to treat a ruptured blister aneurysm with good results [42]. Being a valuable adjunct in blister lesion surgery, encircling clip grafts should be readily available in all such procedures. Sadly, these adjuncts are rarely part of the modern day aneurysm clip tray, and young neurosurgeons are often not even aware of their existence.

Despite all efforts, intraoperative rupture of a blister aneurysm carries a mortality rate of up to $25 \%$ [57]. On this basis, and with surgeons trying for improved results, novel techniques continue to emerge. An interesting addition to our armatorium has been recently proposed by Kazumata et al. in the form of a protective bypass concept. According to these authors, when dealing with a blister aneurysm, and knowing the fragility of such a lesion, surgeons should at least contemplate an STA-MCA bypass prior to any attempted clipping. Ensuring adequate cerebral perfusion, this technique allows, in case of an intraoperative laceration, time for corrective measures to be applied accordingly. The duration of temporary occlusion for direct suturing or clip reapplication seizes to be a limiting factor, while, should the need for a carotid occlusion arise, this can be done safely, combined with a proper high-flow, radial artery graft bypass [58].

\subsubsection{Wrapping procedures}

In an effort to limit intraoperative manipulation of the lesion, neurosurgeons, especially in the past, have widely used wrapping of blister aneurysms as an alternative to clipping mode of treatment. In this context, wrapping is undertaken when a traditional clip cannot be properly applied due to either the morphology of the aneurysm itself or the underlying sclerotic changes of the parent vessel (i.e. the carotid artery) [46]. The technique entails encasing of the entire diseased segment of the carotid artery with an appropriately sized thin sheet of-usually-cotton. On occasions, cuts along its longitudinal axis are made to accommodate for branch vessels and perforators. Creation of ample room to allow free movement of surgical instruments and circumferential dissection of the aneurysm dome can prove dangerous but are, at the same time, essential. The wrapping material is applied with 
the aid of microforceps, and an angled clip is used to fit it snugly around the artery. Once the wrapping is secured, any excess length is cut and removed [39, 41].

The concept behind wrapping is that mechanical reinforcement of the diseased carotid artery provides protection from a potential rerupture during the immediate postoperative period while, in the long term, induced inflammatory changes eventually lead to connective tissue formation and remodeling of the weak arterial wall into a histologically competent structure $[47,59,60]$. Wrapping materials that have been tried out include muscle, muslin gauze, Teflon, silicon, collagen-impregnated Dacron fabric and cotton [46]. The latter seems to dominate today the relevant literature with most authors proposing it as the most effective option. Notably, Biobond, a cyanoacrylate glue widely used in the past, is by now obsolete due to its toxicity [60-62].

Complications associated with wrapping are rare. Perforator injury or parent vessel narrowing following placement of whichever material has been chosen make postoperative angiography an absolute necessity [63]. Delayed development of a granuloma or arachnoiditis has also been reported resulting in cranial neuropathies $[64,65]$, an observation that has led many authors to suggest that cotton must be used with the utmost care and never be placed in contact with adjacent cranial nerves, especially the chiasm $[46,63]$.

In the premicrosurgery era, numerous studies have been published on the efficacy and safety of wrapping. Among these, the largest series has been the one presented by Todd et al. in 1989. According to it, the risk of early (within 6 months) rehemorrhage after wrapping of an aneurysm has been calculated to be $8.6 \%$, while late incidents were at $1.5 \%$ per year [66]. Results seem to have been significantly better after the introduction of the surgical microscope although only few relevant articles exist. Characteristically, Cudlip et al. reported in 1998 a series of 15 wrapped aneurysms with no rehemorrhages within 1 year after surgery [67].

Wrapping is unquestionably inferior to clip ligation for the treatment of ruptured aneurysms. However, it does offer a degree of protection during the immediate postoperative period, and, by doing so, it remains today a valid—even though last resort - therapeutic option when confronted with a blister aneurysm [47].

\subsection{Endovascular treatment}

Initial attempts at endovascular treatment with primary coiling of blister aneurysms (Figure 3) have returned, in most cases, disappointing results [68]. A high risk of intraprocedural rupture and coil protrusion or migration were problems commonly encountered due to the small size and shallow morphology of such lesions, their fragile nature and their proximity to vascular curves on the carotid wall (the latter, in combination with the typical orientation of blister aneurysms, has been known to necessitate a difficult and extremely gentle catheterization maneuver in order to gain access to the sac while avoiding a potential perforation) $[19,35]$. Additionally, the lack, in many cases, of a true wall often allowed for posttreatment progression and-possibly—rebleeding [40]. As a consequence, most authors advocated that blister aneurysms are unsuitable for endovascular treatment and should therefore be left to surgery [69].

With the introduction of intracranial stents in clinical practice, our conception of blister lesion management has gradually changed. Surgical techniques are increasingly looked down upon as outdated and old-fashioned while stent-assisted coiling (Figure 4) became, initially at least, the new trend in the field. The procedure is carried out either by first placing the stent (e.g. Neuroform or Enterprise stents) and then introducing coils through its struts (trans-stent coiling) or by catheterizing the aneurysm sac and deploying the stent over the microcatheter 


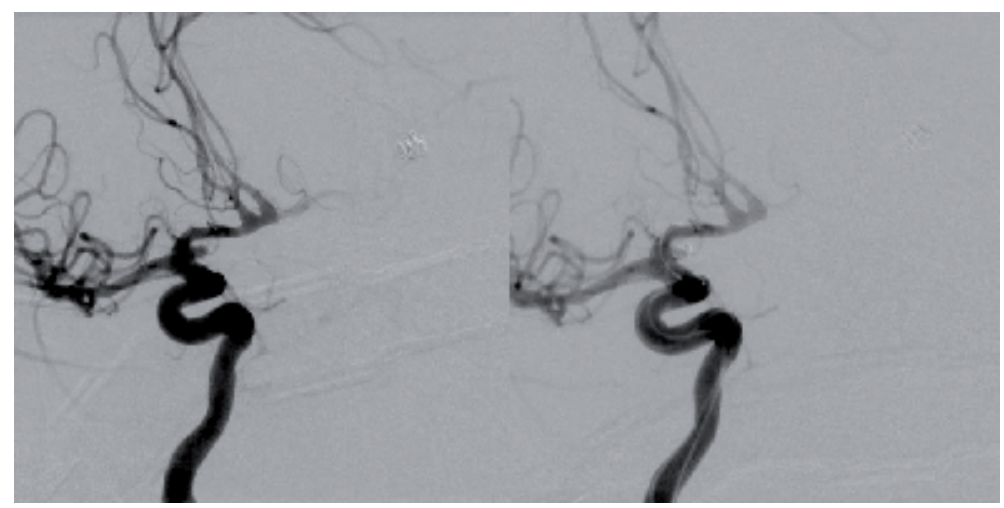

Figure 3.

Simple coiling of a right ICA blister aneurysm. Intraprocedural images.

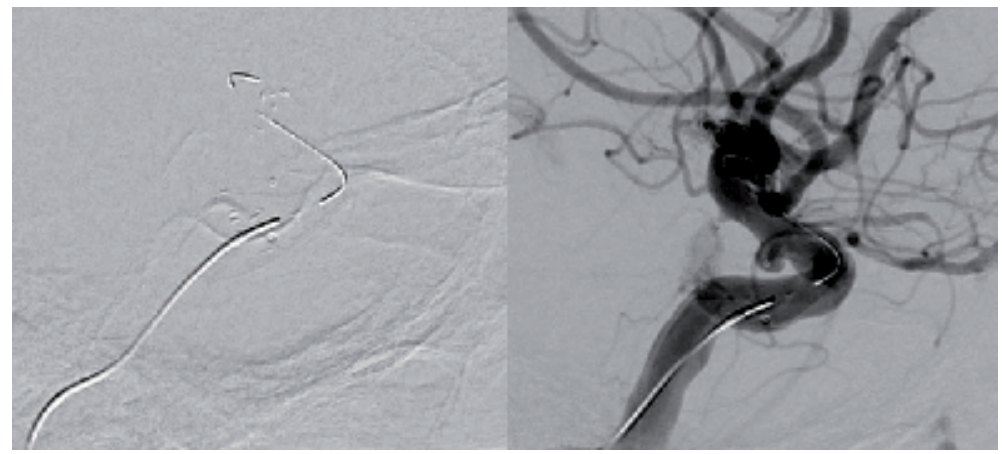

Figure 4.

Intraprocedural images showing stent-assisted coiling of a left ICA blister lesion.

prior to coiling (jailing technique). Facilitating stable intrasaccular coil deployment while at the same time reinforcing the underlying diseased arterial wall, stent-assisted coiling promised to provide a safe and reliable therapeutic alternative $[20,70]$. However, it was soon realized that results, even though better than those of surgery, were far from optimal. Intraoperative complications, mainly bleeding, were encountered in up to $17 \%$ of cases, while the risk for recurrence of the lesion, need for further treatment and postoperative repeat hemorrhage were reported at 65,50 and $13 \%$, respectively $[9,35,71]$. Notably, Meckel et al. found that the latter, a potentially catastrophic and fatal event, is seen only in cases that show incomplete occlusion (i.e. neck remnant or residual sac) by the end of the initial already procedure and especially if the patient receives full double antiplatelet treatment postoperatively or if the aneurysm in question is atypically large or partially thrombosed [35]. In this context, early and tight angiographic follow-up of partially obliterated lesions is essential, while any signs of regrowth should prompt complementary treatment. In the face of all these, most authors are by now moving away from stent-assisted coiling being the preferred primary and sole mode of treatment. Instead, they are using it, if possible, as a preliminary means to achieve a certain degree of protection until definite treatments, in the form of some other techniques, can be instituted.

Prior to moving on, and in order to close the discussion of stent-assisted coiling, it should be noted that one more major argument against it is the need for subsequent antiplatelet therapy [72]. This, combined with a potentially still unsecure aneurysm, requires careful consideration. Additional risks include the frequent 
need for a surgical intervention at a later time (e.g. extraventricular drainage or shunting procedures) $[73,74]$ and delayed spontaneous intraparenchymal bleeding with the rate of the later though not being affected by the initial subarachnoid hemorrhage $[75,76]$. The exact therapeutic antiplatelet regimen that should be used in such a setting is still debatable. Some authors suggest that a similar to elective case scheme should be followed: loading with full doses of aspirin and clopidogrel just prior to the procedure, double antiplatelets for 3-6 months (usually $75 \mathrm{mg}$ aspirin and $75 \mathrm{mg}$ clopidogrel daily) and then continuation of only aspirin for another 6 months to life [77]. Going even further, Lee et al. augmented this regimen by a full-dose heparinization for 24-48 hours postoperatively [1]. On the contrary, other authors favor a reduced or even single-drug scheme. The rationale for this is that in the presence of a high-flow state within the stented lumen of large-size vessels (e.g. internal carotid artery), fibrin formation or clotting is relatively unlikely. Moreover, the risk of and the consequences of a potential intraprocedural rerupture are minimized, while at the same time occlusion of the aneurysm through thrombus formation is significantly facilitated. Using such an approach, Meckel et al. reported in 2011 only 1 fatal rehemorrhage in between 11 patients who were primarily treated with stent-assisted coiling of blister aneurysms. In other reports, stent-assisted coiling under systemic heparinization and loading with dual antiplatelets only after the procedure was found to be associated with a cumulative risk of thrombotic and hemorrhagic complications as low as $2 \%$ [78], while the intraprocedural use of aspirin and later introduction of clopidogrel carried a $21 \%$ overall risk of perioperative complications [79]. In any case, and because of significant heterogeneity of response to antiplatelet drugs, platelet function should be performed prior to any intervention as well as during the postoperative period (a patient's response may be found to change over time) [80,81].

A recent development in blister aneurysm treatment and a welcome addition to our armatorium has been flow diversion. According to advocates of such a concept, and given that blister lesions are regarded by many as pseudoaneurysms, flow diversion is the only endovascular technique capable of actually reconstructing the vessel wall and sealing off any underlying defect $[8,82]$. This can be achieved either through a stent-in-stent technique or with the aid of devices such as the pipeline or the Silk flow diversion systems. The former typically entails telescopic deployment of multiple overlapping stents within the diseased vessel in an effort to increase the total mesh density and thus restore proper parent artery laminar flow [8]. This results in thrombosis of the lesion, an effect augmented by endothelial proliferation along the length of the implanted stents. In the same direction, and specifically designed with this in mind, proper flow diverters like the Silk or the pipeline systems provide significantly better hemodynamic results at the expense however of increased perioperative complications due to their stiffness and thrombogenicity [83-85]. Notably, and regardless of the technique selected, flow diversion allows for preservation of branching vessels, an important feature when it comes to blister aneurysms since such lesions are usually located close to the posterior communicating or the anterior choroidal arteries [86].

Major concerns with the use of flow diversion for the treatment of blister lesions include an even more prominent need for antiplatelets as well as the fact that such an approach does not guarantee protection from postoperative progression and rerupture. Regarding the latter, and despite reports of a marked decrease in intraaneurysmal flow on the intraoperative already angiogram, hemodynamic stress upon the lesion theoretically remains at least for a few days [40]. Rasskazoff et al. recently reported that even with the use of double SILK flow diverters, complete occlusion of a blister aneurysm they treated did not occur till 18 days after the intervention, while Consoli et al. verified obliteration of a similar lesion no less than 
6 months postprocedurally $[8,75]$. A valid alternative possibly addressing the whole issue is the combination of flow diversion with coiling. On this basis, Kim et al. have reported favorable results with stent-assisted coiling as a primary treatment augmented by deployment of a second flow diverting stent if needed (i.e. postoperative progression of the lesion) [39]. In cases with extremely small lesions where coil deployment is perceived as carrying a significant risk, the reverse route can also be followed: telescopic stenting and subsequent trans-stent coiling should the lesion further grow to allow that [40]. Another attractive option involves covered stents; their use however is still limited due-mainly—-to their stiffness, a feature that makes their intracranial delivery not only difficult but also dangerous since they may impinge on the fragile aneurysm neck portion resulting in intraoperative rupture. In addition, they often prove impossible to fully conform to the curved supraclinoid carotid wall, and, in this way, they can potentially leave an underlying aneurysm essentially open [39]. Whatever the case, further experience is needed should such devices gain a significant role in blister aneurysm treatment.

\subsection{Parent artery occlusion}

Although often considered as inherently inferior, deconstructive techniques allow for definitive occlusion of a blister aneurysm with minimal direct manipulation of its walls. In this context, carotid artery sacrifice and trapping of blister lesions significantly reduce the overall risk of rerupture during the perioperative period $[19,87]$.

Should carotid artery occlusion be contemplated as a final treatment, endovascular rather than surgical trapping is recommended due to its convenience, rapidity and safety. In an often cited article, Park et al. have described an elegant technique entailing the use of two microcatheters and a proximal balloon to control blood flow intraoperatively [19]. The first microcatheter is placed distal to the aneurysm, while the second is positioned just at the level of its neck. With the aid of the latter, and after inflation of the balloon, a framing coil is deployed inside the carotid artery. Prior to detachment, the distal microcatheter is retrieved and repositioned within the coil mess. Additional coils are deployed till a stable result is achieved. The use of trapping coils instead of detachable balloons is perceived as having a lesser risk of intraprocedural rupture.

When occlusion of the carotid artery is suggested, postoperative patency of its cardinal branches requires careful consideration. A detailed study of the ophthalmic, the posterior communicating and the anterior choroidal arteries as well as their collaterals is essential:

\section{i. Ophthalmic artery}

Blister aneurysms arise on the communicating segment of the internal carotid artery, and therefore the ophthalmic artery does not usually present a problem when dealing with such a lesion. If a choroidal blush via the external carotid circulation is visualized on preoperative angiograms, the trapped segment of the carotid artery is typically extended to include the origin of the ophthalmic artery as well, achieving thus a more robust result. As an additional precaution, proximal occlusion of the cervical internal carotid artery may also be performed. If, on the other hand, no ophthalmic collateral vessels are visualized, preservation of the ophthalmic artery is absolutely essential, and carotid trapping should be kept short. Accordingly, the proximal carotid artery cannot be occluded, and, as a consequence, close follow-up is mandatory to exclude recanalization of the trapped segment. 


\section{ii. Posterior communicating artery}

Due to its proximity to blister lesions, the posterior communicating artery often needs to be included in the trapped carotid segment. In most cases this is safe with the only exception being a fetal-type vessel. In the latter case, an alternative therapeutic strategy should be considered.

\section{iii. Anterior choroidal artery}

Preservation of the anterior choroidal artery is essential in order to avoid postoperative hemiplegia. On this basis, precision of segmental occlusion offers obvious advantages. Should the origin of the anterior choroidal artery prove impossible to save, the procedure should be abandoned.

Apart from carotid side branches, another important concern with all deconstructive procedures is postoperative early or late ischemia. Unselective, abrupt occlusion of the internal carotid artery is known to carry a $26 \%$ risk of cerebral infarction and $12 \%$ risk of death [88]. A balloon occlusion test should always be performed when such an approach is contemplated. This entails inflation of a nondetachable balloon at the site of the intended occlusion and subsequent clinical and angiographic evaluation of hemispheric collateral circulation [89, 90]. However, one should always keep in mind that -in the setting of a subarachnoid hemorrhage-results of a balloon occlusion test can be misleading. This is due to the fact that a balloon occlusion test does not take into account the hemodynamic effects of a posthemorrhagic vasospasm which may complicate such cases [16].

For patients who fail a balloon test occlusion, surgical bypass should be contemplated. Traditionally, this is in the form of an artificial communication between the superficial temporal and the middle cerebral artery with or without an interposed vascular graft (radial artery or saphenous vein) (STA-MCA bypass) $[16,58]$. In the case a graft is to be used, this is termed as a high-flow bypass and ensures significantly better results [91]. As a matter of fact, there are authors recommending high-flow bypasses as a primary mode of treatment for blister aneurysms. However, experts in the field seem skeptical advocating that a high-flow bypass should never be thought of as complete substitute for normal carotid artery supply, quoting an $80 \%$ rate of postoperative complications, including graft occlusion and vasospasmrelated cerebral infarction [41]. Additionally, surgical bypass in patients with severely atherosclerotic vessels typically requires antiplatelets, a feature that only adds to the overall risk.

\section{Conclusion}

Our understanding of blister aneurysms is still today incomplete. Having a relatively wide spectrum of pathological differentiations, they can be classified as either true or false lesions. Whether this represents consecutive stages of the same entity or a different, in each case, disease remains to be clarified. Further research on the field is an absolute necessity, and young physicians should thus be encouraged toward this direction by their senior colleagues.

A direct consequence of our limited knowledge on the nature of blister aneurysms is the lack of an established and universally accepted treatment modality. This lack of consensus has led to numerous attempts at novel and often promising therapies. However, and with the sole exception of the recently introduced flow diverters, all previous options have invariably failed to rise to initial expectations. 
Flow diversion, despite its limitations, arises today as probably the most attractive future prospect. However, until our technology reaches that point, blister aneurysms are still to be treated on a case-by-case basis. In this context, when confronted with such a lesion, physicians should consider all available alternatives, both surgical and endovascular, in order to maximize the chances of a good outcome.

Whichever end treatment modality is to be used, an important point when dealing with a patient that has suffered rupture of a blister aneurysm is amelioration of perihemorrhage management in its entity. Careful consideration of all measures to prevent and, if needed, manage rebleeding or any other related complications (i.e. vasospasm, hydrocephalus, seizures) is essential. All these issues would preferably be addressed within a multidisciplinary team consisting of neurosurgeons, interventionists and ICU specialists should the best possible result be achieved.

As with every other such case, and despite initial enthusiasm, each new therapeutic approach proposed for blister lesions needs careful consideration and long periods of follow-up to evaluate its efficacy, safety and durability of results. It is obvious that further research in the field is an absolute necessity and young physicians should be encouraged toward this direction.

\section{Author details}

Themistoklis I. Papasilekas*, Konstantinos M. Themistoklis, Stefanos I. Korfias and Damianos E. Sakas

Department of Neurosurgery, National and Kapodistrian University of Athens, Evaggelismos General Hospital, Athens, Greece

*Address all correspondence to: th.papasilekas@gmail.com

IntechOpen

(C) 2019 The Author(s). Licensee IntechOpen. This chapter is distributed under the terms of the Creative Commons Attribution License (http://creativecommons.org/licenses/ by/3.0), which permits unrestricted use, distribution, and reproduction in any medium, provided the original work is properly cited. (cc) BY 


\section{References}

[1] Lee B-H, Kim BM, Park MS, et al. Reconstructive endovascular treatment of ruptured blood blister-like aneurysms of the internal carotid artery. Journal of Neurosurgery. 2009;110:431-436

[2] Sundt TM, Murphey F. Clip-grafts for aneurysm and small vessel surgery. Journal of Neurosurgery. 1969;31:59-71

[3] Nakagawa F, Kobayashi S, Takemae T, et al. Aneurysms protruding from the dorsal wall of the internal carotid artery. Journal of Neurosurgery. 1986;65:303-308

[4] Ogawa A, Suzuki M, Ogasawara K. Aneurysms at nonbranching sites in the surpaclinoid portion of the internal carotid artery: Internal carotid artery trunk aneurysms. Neurosurgery. 2000;47:578-583; discussion 583-6

[5] Gibo H, Lenkey C, Rhoton AL. Microsurgical anatomy of the supraclinoid portion of the internal carotid artery. Journal of Neurosurgery. 1981;55:560-574

[6] Sim SY, Shin YS, Cho KG, et al. Blood blister-like aneurysms at nonbranching sites of the internal carotid artery. Journal of Neurosurgery. 2006;105:400-405

[7] Seo D-H, Lee W-C, Choe I-S, et al. Ruptured and unruptured aneurysms of the accessory anterior cerebral artery combined with a blood blister-like aneurysm of the anterior communicating artery. Neurology India. 2009;57:85-87

[8] Consoli A, Nappini S, Renieri L, et al. Treatment of two blood blister-like aneurysms with flow diverter stenting. Journal of NeuroInterventional Surgery. 2012;4:e4-e4

[9] Gonzalez AM, Narata AP, Yilmaz H, et al. Blood blister-like aneurysms:
Single center experience and systematic literature review. European Journal of Radiology. 2014;83:197-205

[10] Sano K. Concerning the nomenclature and classification of internal carotid aneurysms. Surgery for Cerebral Stroke. 2014;24:333-339

[11] Abe M, Tabuchi K, Yokoyama H, et al. Blood blisterlike aneurysms of the internal carotid artery. Journal of Neurosurgery. 1998;89:419-424

[12] Stehbens WE. Pathology of the Cerebral Blood Vessels. Saint Louis: C.V. Mosby; 1972

[13] Satoh A, Nakamura H, Odaki M, et al. High risk aneurysm of the internal carotid artery. Surgery for Cerebral Stroke. 2014;21:467-472

[14] Çinar C, Oran İ, Bozkaya H, et al. Endovascular treatment of ruptured blister-like aneurysms with special reference to the flow-diverting strategy. Neuroradiology. 2013;55:441-447

[15] Yaşargil MG. Microneurosurgery. Georg Thieme. 1984. Available from: http://www.thieme.co.uk/ microneurosurgery-volume-ii [Accessed: 06 August 2019]

[16] Meling TR, Sorteberg A, Bakke SJ, et al. Blood blister-like aneurysms of the internal carotid artery trunk causing subarachnoid hemorrhage: Treatment and outcome. Journal of Neurosurgery. 2008;108:662-671

[17] Shigeta H, Kyoshima K, Nakagawa F, et al. Dorsal internal carotid artery aneurysms with special reference to angiographic presentation and surgical management. Acta Neurochirurgica. 1992;119:42-48

[18] Jha AN, Gupta V. Blister aneurysms. Neurology India. 2009;57:2-3 
[19] Park JH, In Sung P, Han DH, et al. Endovascular treatment of blood blister-like aneurysms of the internal carotid artery. Journal of Neurosurgery. 2007;106:812-819

[20] Oktar N, Oran I, Özdamar N, et al. Blister aneurysms. Journal of the Neurological Sciences. 2009;26:115-119

[21] Bradley WG, Walter G. Neurology in Clinical Practice. Philadelphia:

Butterworth-Heinemann; 2000

[22] Shennan T. Dissecting Aneurysms. Special Report Series No. 193. London; 1934

[23] Suzuki J, Ohara H.

Clinicopathological study of cerebral aneurysms. Origin, rupture, repair, and growth. Journal of Neurosurgery. 1978;48:505-514

[24] Charbel FT, Gonzales-Portillo G, Hoffman W, et al. Distal internal carotid artery pseudoaneurysms: Technique and pitfalls of surgical management: Two technical case reports. Neurosurgery. 1999;45:643-648; discussion 648-9

[25] Ishikawa T, Nakamura N, Houkin K, et al. Pathological consideration of a "blister-like"; aneurysm at the superior wall of the internal carotid artery: Case report. Neurosurgery. 1997;40:403-405; discussion 405-6

[26] Kim J-H, Kwon T-H, Kim J-H, et al. Internal carotid artery dorsal wall aneurysm with configurational change: Are they all false aneurysms? Surgical Neurology. 2006;66:441-443; discussion 443

[27] Ohara H, Sakamoto T, Suzuki J. Clinical study of 11 cases of cerebral aneurysms diagnosed sclerotic origin--sclerotic cerebral aneurysms (author's transl). No Shinkei Geka. 1978;6:1057-1064
[28] Stehbens WE. Etiology of intracranial berry aneurysms. Journal of Neurosurgery. 1989;70:823-831

[29] Glynn LE. Medial defects in the circle of Willis and their relation to aneurysm formation. The Journal of Pathology and Bacteriology. 1940;51:213-222

[30] Stanson AW, Kazmier FJ, Hollier LH, et al. Penetrating atherosclerotic ulcers of the thoracic aorta: Natural history and clinicopathologic correlations. Annals of Vascular Surgery. 1986;1:15-23

[31] Forsting M, Wanke I, editors. Intracranial Vascular Malformations and Aneurysms. Berlin, Heidelberg: Springer Berlin Heidelberg; 2008. DOI: 10.1007/978-3-540-32920-6

[32] van der Wee N, Rinkel GJ, Hasan D, et al. Detection of subarachnoid haemorrhage on early CT: Is lumbar puncture still needed after a negative scan? Journal of Neurology, Neurosurgery, and Psychiatry. 1995;58:357-359

[33] Gaughen JR, Raghavan P, Jensen ME, et al. Utility of CT angiography in the identification and characterization of supraclinoid internal carotid artery blister aneurysms. AJNR. American Journal of Neuroradiology. 2010;31:640-644

[34] Le Feuvre DEJ, Taylor AG. The management of very small/blister internal carotid artery aneurysms. Interventional Neuroradiology. 2011;17:431-434

[35] Meckel S, Singh TP, Undrén P, et al. Endovascular treatment using predominantly stent-assisted coil embolization and antiplatelet and anticoagulation management of ruptured blood blister-like aneurysms. AJNR. American Journal of Neuroradiology. 2011;32:764-771 
[36] White PM, Wardlaw JM, Easton V. Can noninvasive imaging accurately depict intracranial aneurysms? A systematic review. Radiology. 2000;217:361-370

[37] Wintermark M, Uske A, Chalaron M, et al. Multislice computerized tomography angiography in the evaluation of intracranial aneurysms: A comparison with intraarterial digital subtraction angiography. Journal of Neurosurgery. 2003;98:828-836

[38] Tekkök IH, Bakar B. Ruptured blister-like aneurysm of distal internal carotid artery: A distinct entity. Turkish Neurosurgery. 2008;18:439-445

[39] Kim BM, Chung EC, Il PS, et al. Treatment of blood blister-like aneurysm of the internal carotid artery with stent-assisted coil embolization followed by stent-within-a-stent technique. Case report. Journal of Neurosurgery. 2007;107:1211-1213

[40] Gaughen JR, Hasan D, Dumont AS, et al. The efficacy of endovascular stenting in the treatment of supraclinoid internal carotid artery blister aneurysms using a stent-in-stent technique. AJNR. American Journal of Neuroradiology. 2010;31:1132-1138

[41] Garrett M, Spetzler RF. Surgical treatment of blister-like aneurysms. World Neurosurgery. 2012;77:76-77

[42] Cho TG, Hwang SN, Nam TK, et al. Salvage surgical treatment for failed endovascular procedure of a blood blister-like aneurysm. Journal of Cerebrovascular and Endovascular Neurosurgery. 2012;14:99-103

[43] Joo S-P, Kim T-S, Moon K-S, et al. Arterial suturing followed by clip reinforcement with circumferential wrapping for blister-like aneurysms of the internal carotid artery. Surgical
Neurology. 2006;66:424-428; discussion 428-9

[44] Karasawa H, Matsumoto H, Naito H, et al. Superior wall aneurysms of the internal carotid artery: Intraoperative findings and operative technique. Japanese Journal of Neurosurgery. 1996;5:273-280

[45] Papasilekas TI, Themistoklis KM, Andreou AA. Current trends in the surgical management of blister aneurysms. An illustrative case series. Clinical Neurology and Neurosurgery. 2018;168:54-59

[46] Deshmukh VR, Kakarla UK, Figueiredo EG, et al. Long-term clinical and angiographic follow-up of Unclippable wrapped intracranial aneurysms. Neurosurgery. 2006;58:434-442

[47] Kim YB, Hong CK, Chung J, et al. Long-term clinical and angiographic outcomes of wrap-clipping strategies for unclippable cerebral aneurysms. Yonsei Medical Journal. 2014;55:401-409

[48] Ogilvy CS. Repair of an arterial perforation of the internal carotid artery using Hemashield wrapping with aneurysm clip reinforcement: Technical note. Neurosurgery. 1997;40:1312-1314

[49] Takahashi A, Suzuki J, Fujiwara S, et al. Surgical treatment of Chimame (blood blister) like aneurysm at C2 portion of internal carotid artery. Surgery for Cerebral Stroke. 1988;16:72-77

[50] Regelsberger J, Matschke J, Grzyska U, et al. Blister-like aneurysms--a diagnostic and therapeutic challenge. Neurosurgical Review. 2011;34:409-416

[51] Sasaki O, Ogawa H, Koike T, et al. A clinicopathological study of dissecting aneurysms of the intracranial vertebral 
artery. Journal of Neurosurgery. 1991;75:874-882

[52] Takeshita M, Onda H, Tanikawa T, et al. Clinical analysis of the aneurysms of the Anterior Wall of the intracranial internal carotid artery. Surgery for Cerebral Stroke. 1997;25:134-139

[53] Okuchi K, Fujioka M, Maeda Y, et al. Surgical treatment of internal carotid artery Anterior Wall aneurysm with extravasation during angiography. Neurologia Medico-Chirurgica. 1999;39:40-44

[54] Yanaka K, Meguro K, Nose T. Repair of a tear at the base of a blisterlike aneurysm with suturing and an encircling clip: Technical note. Neurosurgery. 2002;50:218-221

[55] Sundt T. Surgical Techniques for Saccular and Giant Intracranial Aneurysms. Baltimore: Williams \& Wilkins; 1990. DOI: $10.1016 /$ s0025-6196(12)60963-x

[56] Sekula RF, Cohen DB, Quigley MR, et al. Primary treatment of a blisterlike aneurysm with an encircling clip graft: Technical case report. Operative Neurosurgery. 2006;59:ONSE168

[57] Satoh A, Hongo K, Sugiyama T, Ishihara S, Yamane F, Kakizawa Y. The Nationwide surveillance on the dorsal aneurysm of the internal carotid artery part 2: Study on the surgical treatment in hemorrhagic cases. Surgery for Cerebral Stroke. 2006;34:372-376

[58] Kazumata K, Nakayama N, Nakamura T, et al. Changing treatment strategy from clipping to radial artery graft bypass and parent artery sacrifice in patients with ruptured blister-like internal carotid artery aneurysms. Neurosurgery. 2014;10(Suppl 1):66-72; discussion 73

[59] Mount LA, Antunes JL. Results of treatment of intracranial aneurysms by wrapping and coating. Journal of Neurosurgery. 1975;42:189-193

[60] Herrera O, Kawamura S, Yasui N, et al. Histological changes in the rat common carotid artery induced by aneurysmal wrapping and coating materials. Neurologia Medico-Chirurgica. 1999;39:134-139; discussion 139-40

[61] Kurita H, Shiokawa Y, Segawa H, et al. Delayed parent artery narrowing occurring months after aneurysm surgery: A complication after aneurysm surgery--technical case report. Neurosurgery. 1995;36:1225-1229

[62] Juan GM, Kawamura S, Yasui N, et al. Histological changes in the rat common carotid artery following simultaneous topical application of cotton sheet and cyanoacrylate glue. Neurologia Medico-Chirurgica. 1999;39:908-911; discussion 912

[63] Haisa T, Matsumiya K, Yoshimasu N, et al. Foreign-body granuloma as a complication of wrapping and coating an intracranial aneurysm. Case report. Journal of Neurosurgery. 1990;72:292-294

[64] McFadzean RM, Hadley DM, McIlwaine GG. Optochiasmal arachnoiditis following muslin wrapping of ruptured anterior communicating artery aneurysms. Journal of Neurosurgery. 1991;75: 393-396

[65] Onoue H, Abe T, Tashibu K, et al. Two undesirable results of wrapping of an intracranial aneurysm. Neurosurgical Review. 1992;15:307-309

[66] Todd NV, Tocher JL, Jones PA, et al. Outcome following aneurysm wrapping: A 10-year follow-up review of clipped and wrapped aneurysms. Journal of Neurosurgery. 1989;70:841-846

[67] Cudlip SA, Kitchen ND, McKhahn GM, et al. Wrapping 
of solitary ruptured intracranial aneurysms, outcome at five years. Acta Neurochirurgica. 1998;140:1167-1170; discussion 1170-1

[68] McNeely PD, Clarke DB, Baxter B, et al. Endovascular treatment of a "blister-like" aneurysm of the internal carotid artery. The Canadian Journal of Neurological Sciences. 2000;27:247-250

[69] Greenberg MS. Handbook of Neurosurgery. Tampa: Greenberg Graphics; 1997

[70] Ahn JY, Cho JH, Jung JY, et al. Blister-like aneurysms of the supraclinoid internal carotid artery: Challenging endovascular treatment with stent-assisted coiling. Journal of Clinical Neuroscience. 2008;15:1058-1061

[71] Fang Y-B, Li Q, Yang P-F, et al. Treatment of blood blister-like aneurysms of the internal carotid artery with stent-assisted coil embolization. Clinical Neurology and Neurosurgery. 2013;115:920-925

[72] Ihn YK, Kim SH, Sung JH, et al. The efficacy of endovascular treatment of ruptured blood blister-like aneurysms using stent-assisted coil embolization. Interventional Neuroradiology. 2012;18:432-441

[73] Kung DK, Policeni BA, Capuano AW, et al. Risk of ventriculostomy-related hemorrhage in patients with acutely ruptured aneurysms treated using stent-assisted coiling. Journal of Neurosurgery. 2011;114:1021-1027

[74] Mahaney KB, Chalouhi N, Viljoen S, et al. Risk of hemorrhagic complication associated with ventriculoperitoneal shunt placement in aneurysmal subarachnoid hemorrhage patients on dual antiplatelet therapy. Journal of Neurosurgery. 2013;119:937-942
[75] Rasskazoff S, Silvaggio J, Brouwer P, et al. Endovascular treatment of a ruptured blood blister-like aneurysm with a flow-diverting stent. Interventional Neuroradiology. 2010;16:255-258

[76] Martin AR, Cruz JP, Matouk CC, et al. The pipeline flow-diverting stent for exclusion of ruptured intracranial aneurysms with difficult morphologies. Neurosurgery. 2012;70:21-28; discussion 28

[77] Faught RWF, Satti SR, Hurst RW, et al. Heterogeneous practice patterns regarding antiplatelet medications for neuroendovascular stenting in the USA: A multicenter survey. Journal of NeuroInterventional Surgery. 2014;6:774-779

[78] Katsaridis V, Papagiannaki C, Violaris C. Embolization of acutely ruptured and unruptured widenecked cerebral aneurysms using the neuroform 2 stent without pretreatment with antiplatelets: A single center experience. AJNR. American Journal of Neuroradiology. 2006;27:1123-1128

[79] Tähtinen OI, Vanninen RL, Manninen HI, et al. Wide-necked intracranial aneurysms: Treatment with stent-assisted coil embolization during acute. Radiology. 2009;253:199-208

[80] Delgado Almandoz JE, Crandall BM, Scholz JM, et al. Last-recorded $\mathrm{P} 2 \mathrm{Y} 12$ reaction units value is strongly associated with thromboembolic and hemorrhagic complications occurring up to 6 months after treatment in patients with cerebral aneurysms treated with the pipeline embolization device. American Journal of Neuroradiology. 2014;35:128-135

[81] Sibbing D, Byrne RA, Bernlochner I, et al. High platelet reactivity and clinical outcome - fact and fiction. Thrombosis and Haemostasis. 2011;106:191-202 
[82] Kulcsár Z, Wetzel SG, Augsburger L, et al. Effect of flow diversion treatment on very small ruptured aneurysms. Neurosurgery. 2010;67:789-793

[83] Wagner A, Cortsen M, Hauerberg J, et al. Treatment of intracranial aneurysms. Reconstruction of the parent artery with flowdiverting (silk) stent. Neuroradiology. 2012;54:709-718

[84] Cruz JP, O’Kelly C, Kelly M, et al. Pipeline embolization device in aneurysmal subarachnoid hemorrhage. American Journal of Neuroradiology. 2013;34:271-276

[85] Chalouhi N, Zanaty M, Tjoumakaris S, et al. Treatment of blister-like aneurysms with the pipeline embolization device. Neurosurgery. 2014;74:527-532

[86] Causin F, Pascarella R, Pavesi G, et al. Acute endovascular treatment (<48 hours) of uncoilable ruptured aneurysms at non-branching sites using silk flow-diverting devices. Interventional Neuroradiology. 2011;17:357-364

[87] Başkaya MK, Ahmed AS, Ateş Ö, et al. Surgical treatment of blood blister-like aneurysms of the supraclinoid internal carotid artery with extracranial-intracranial bypass and trapping. Neurosurgical Focus. 2008;24:E13

[88] Linskey ME, Jungreis CA, Yonas H, et al. Stroke risk after abrupt internal carotid artery sacrifice: Accuracy of preoperative assessment with balloon test occlusion and stable xenonenhanced CT. AJNR. American Journal of Neuroradiology. 1994;15:829-843

[89] Mathis JM, Barr JD, Jungreis CA, et al. Temporary balloon test occlusion of the internal carotid artery: Experience in 500 cases. AJNR. American Journal of Neuroradiology. 1995;16:749-754

[90] Abud DG, Spelle L, Piotin M, et al. Venous phase timing during balloon test occlusion as a criterion for permanent internal carotid artery sacrifice. AJNR. American Journal of Neuroradiology. 2005;26:2602-2609

[91] Murai Y, Mizunari T, Umeoka K, et al. Ischemic complications after radial artery grafting and aneurysmal trapping for ruptured internal carotid artery anterior wall aneurysm. World Neurosurgery. 2012;77:166-171 
Section 4

Arteriovenous Fistulas 



\title{
Arteriovenous Fistulas: The Pathological Bridge
}

\author{
Sara A. AbuSini, Nour A. Tashtush, Abdelwahab J. Aleshawi \\ and Amer A. Jaradat
}

\begin{abstract}
An intracranial dural arteriovenous fistula (DAVF) is a type of intracranial vascular malformation in which there is a connection between an intracranial artery and a dural venous sinus. It accounts for $10-15 \%$ of all intracranial arteriovenous malformations. This malformation derives its arterial supply primarily from meningeal vessels, and the venous drainage is either via dural venous sinuses or through the cortical veins. DAVFs have a reported association with dural sinus thrombosis, venous hypertension, previous craniotomy, and trauma, though many lesions are idiopathic. Digital subtraction angiography remains the gold standard for diagnosing these fistulas. Endovascular treatment is one of the first line options available for their management. In this chapter, we will discuss and review the etiopathogenesis, natural history, common classification systems, and various available diagnostic options.
\end{abstract}

Keywords: angiography, fistula, intervention, pathophysiology

\section{Introduction}

DAVFs are arteriovenous shunts from a dural arterial supply to a dural venous channel, typically supplied by pachy-meningeal arteries and located near a major venous sinus. The etiology of these lesions is not fully understood; some are congenital, and others are acquired. DAVFs in the pediatric population are associated with structural venous abnormalities [1], but most DAVFs are thought to be acquired. Different etiologies have been implicated in this phenomenon, namely sinus thrombosis, trauma, or surgery [2-6].

Yasargill noted that Rizzoli, in 1881, was the first to describe an arteriovenous malformation (AVM) that involved the dura mater and Sachs reported the first, angiographic description in 1931. Subsequently cranial dural fistulas have been most frequently described at the transverse sinus and cavernous sinus, although they occur at every cranial dural sinus. Dural arteriovenous fistulas (DAVFs) can occur anywhere within the intracranial dura mater. DAVFs are rare vascular abnormalities. They consist of numerous tiny connections between branches of dural arteries and veins or a venous sinus [7]. The true incidence of DAVFs is unknown [8]. However, the reported incidence of intracranial DAVFs is approximately $10-15 \%$ of all intracranial vascular abnormalities. A good percentage of DAVFs are asymptomatic or undergo spontaneous involution therefore the true incidence may be much more [9]. DAVFs are acquired lesions; that is why they present later in life than AVMs [10]. 


\section{Pathogenesis}

Many factors have been involved in the pathogenesis of DAVFs; however, the exact pathophysiology remains unclear.

Some patients with DAVFs have been found to have venous sinus occlusion, which was proposed to be the direct cause for the development of the fistula. While others were found to have venous sinus thrombosis which resulted in the formation of small dural arteriovenous shunts by the invasion of angiogenic factors, released from the organizing thrombus, to a group of small dural arteries. However, it has been noted that DAVF can precede either venous sinus occlusion or thrombosis, because of its association with venous hypertension $[11,12]$.

Venous hypertension may lead to chronic focal decrease in both regional cerebral blood flow and cerebral perfusion pressure. The decrease in the cerebral blood flow will cause cerebral ischemia, which increases the expression of vascular endothelial growth factor (VEGF) via hypoxia-inducible factor-1 $\alpha$ (HIF-1 $\alpha)$ upregulation, leading eventually to the formation of DAVF. Also, some theories suggest that if venous hypertension was present, a small arteriovenous anastomosis might open up and generates DAVF when the shunting enlarges [13].

Besides occurring incidentally, the development of DAVFs has been caused by a wide range of events with head trauma, with or without skull fractures, being the most common one. Other preceding events include surgical operations like craniotomy, hormonal alterations like what happens in cases of pregnancy and menopause, infections like cases of otitis and sinusitis, and tumors, particularly meningiomas [14].

\section{Classification}

The Borden-Shucart and Cognard systems are both known to be the most commonly used systems to classify DAVFs. However, there have been many classification schemes submitted for DAVF [10]. The Borden classification system (Table 1) classified DAVFs into three main types according to the presence or absence of cortical venous drainage (CVD) and the location of venous drainage [15]. In type I lesion, the drainage goes to the dural sinus or meningeal vein in an anterograde fashion. In type II lesion, the drainage to dural sinus goes in an anterograde fashion, however, the high pressure within the DAVF causes blood to flow in a retrograde way to subarachnoid veins. In type III lesions, the drainage goes directly and completely to subarachnoid veins [11].

The Cognard grading scale (Table 2) classifies DAVFs into five main types based on the direction of the flow whether anterograde or retrograde, presence or absence of a CVD, presence of venous ectasia whether ectatic or nonectatic cortical vein and the location of the fistula [14].

The presence of a CVD (Borden type II and III, Cognard types IIb-V) or absence (Borden type I, Cognard types I, IIa) and the pattern of the venous drainage all are considered major factors by the previous classification systems and were used to assess the risk of hemorrhage or any neurologic deficits [16]. The absence of a CVD

\begin{tabular}{cl}
\hline Type 1 & Venous drainage into a dural sinus without CVD \\
\hline Type 2 & Venous drainage into a dural sinus with associated CVD \\
\hline Type 3 & Drainage into cortical veins (CVD) \\
\hline
\end{tabular}

Table 1.

Borden classification of DAVF. 
Arteriovenous Fistulas: The Pathological Bridge

DOI: http://dx.doi.org/10.5772/intechopen.89724

\begin{tabular}{ll}
\hline Type I & Venous drainage into dural sinus with antegrade flow \\
\hline Type II a & Venous drainage into dural sinus with retrograde flow \\
\hline Type II b & Venous drainage into dural sinus with antegrade flow and CVD \\
\hline Type II a $+\mathrm{b}$ & Venous drainage into dural sinus with retrograde flow \& CVD \\
\hline Type III & Venous drainage into cortical veins (CVD) \\
\hline Type IV & CVD with associated venous ectasia(s) \\
\hline Type V & Venous drainage into spinal perimedullary veins \\
\hline
\end{tabular}

Table 2.

Cognard classification of DAVF.

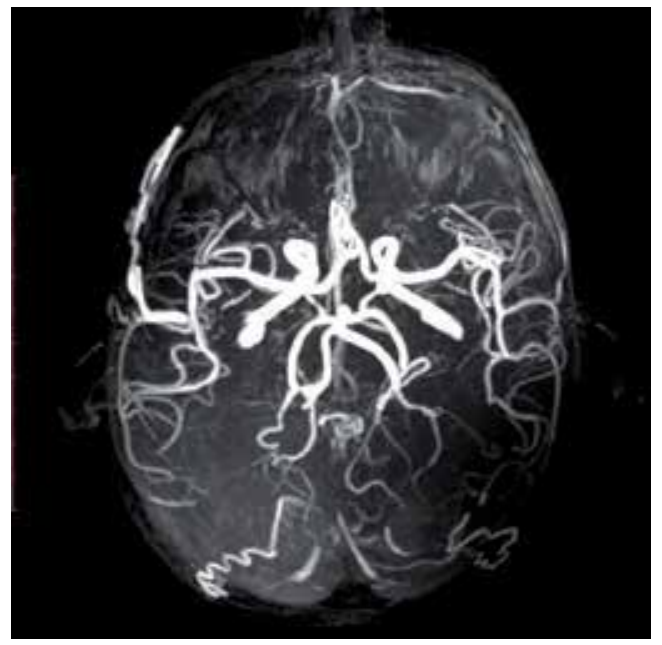

Figure 1.

Magnetic resonance angiography demonstrating the arteriovenous fistula.

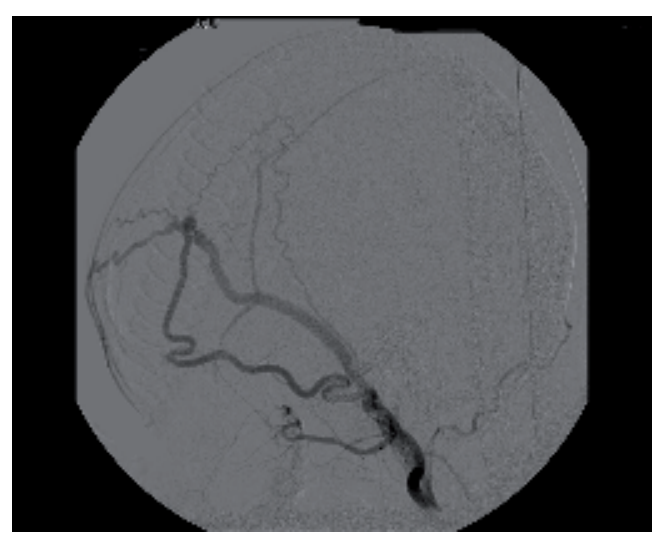

Figure 2.

Digital subtraction angiography showed dural arterio-venous fistula with pseudo aneurysm at the abnormal anastomoses between the right temporal artery and the superior sagittal sinus and the facial vein.

has been recognized as a favorable factor and the patients are usually asymptomatic or have symptoms like pulsatile tinnitus or exophthalmos [15]. On the other hand, presence of a CVD is an unfavorable factor that puts the dural fistula in a higher 
risk group. DAVF has a higher chance of being symptomatic if the type of either classification systems was higher. Also, symptomatic lesions have a higher risk of hemorrhage than asymptomatic lesions [16] (Figures 1 and 2).

\section{Clinical presentation}

Patients with DAVFs may be discovered incidentally and be totally asymptomatic. However, if symptoms are present, they can range widely from mild to fatal in severe cases. The site of DAVF and the direction of the venous drainage (anterograde or retrograde) are two of the factors that will determine the nature of symptoms [17].

Benign symptoms like headache and pulsatile tinnitus present when DAVF drains into dural sinus in an anterograde pattern without CVD. Aggressive symptoms of pseudotumor cerebri, papilledema and dementia present when DAVF drains in a retrograde pattern leading to an increase in the intracranial pressure, hence the severe presentation [11].

According to location, ophthalmic symptoms like chemosis, exophthalmos, double vision and low visual acuity occur if the DAVF is located in the cavernous sinus because the ophthalmic venous drainage may get compromised with subsequent increase in the intraocular pressure and periorbital edema [13]. Ear symptoms like pulsatile tinnitus and retroauricular pain present if DAVF involves transverse and sigmoid sinuses. Pulsatile tinnitus can be heard over the mastoid area with auscultation [16].

Tentorial and anterior cranial fossa DAVFs usually present with hemorrhage because both can obtain retrograde CVD. Exophthalmos and chemosis are also present in cases of anterior cranial fossa DAVFs if they obtain cavernous sinus drainage [11].

In severe cases, patients suffer from intracranial hemorrhage and neurological deficits like seizures, parkinsonism, and cerebellar symptoms [15].

Other presentations include brainstem infarction and cervical myelopathy if DAVF is located around the brainstem. Also, these lesions can cause cranial neuropathy because of the compression on cranial nerves [13].

\section{Diagnosis}

Catheter angiography is the gold standard technique for the diagnosis and classification of DAVFs. Based on the site of DAVF, this method is usually performed by using the four-vessel technique injections with an injection into the external carotid arteries. Several dural arterial feeders with early drainage into a dural sinus or cortical vein are mostly revealed on angiograms. Also, conventional angiograms are used to classify DAVFs based on the venous architecture and the extent of cortical venous reflux.

Among all the used methods for the diagnosis of DAVFs like CT, CT angiography, MRI and MR angiography, CT has been the initial neuroimaging method. Although it can reveal areas of ischemia, edema and $\mathrm{ICH}$, it could not identify DAVF ideally. On the other hand, CTA has been a better method for identifying DAVFs, because it can show a dilated vessel associated with an ICH or calcification with chronically congested veins. Also, MRA has the same ability of CTA of showing dilated vessels.

As both MRA and CTA can show the site of DAVFs in relation to normal brain structures, they are both used as adjuvant methods to catheter angiography, which allows both techniques to be used in intraoperative navigation $[14,15]$. 


\section{Rare cases}

\subsection{Case 1}

This is the first case of a DAVF causing isolated intraventricular hemorrhage with radiographic evidence of several cortical venous refluxes into the subependymal venous network. Also, it is the first case of DAVF that was successfully cured using multimodality transvenous and trans-arterial embolization techniques. A hypertensive, diabetic 84-year-old woman whose modified Rankin scale score is 0 , presented to the emergency room complaining of headache for 4 days, nausea, vomiting and worsening confusion with episodes of severe drowsiness. Noncontrast CT scan of the head, CT angiogram of the brain, and digital subtraction cerebral angiogram were performed. She was treated by transvenous coil embolization of the right transverse/sigmoid sinus using POD5 and penumbra -400 coils and trans-arterial onyx- 18 embolization of the right middle meningeal artery. She had 4- and 6-week follow-up by diagnostic angiogram and 6-week follow-up by clinical examination. The angiogram 6-week follow-up revealed that the DAVF went through complete resolution; the clinical 6-week follow-up showed that the patient mental status returned to normal and her headache resolved [18].

\subsection{Case 2}

A rare case of dural arteriovenous fistula at the cranio-cervical junction presented as cerebellar hemorrhage, which made physicians struggle with the diagnosis. A 77-year-old woman came to the emergency department complaining of dizziness, she had no history of arterial hypertension. A small cerebellar hemorrhage was revealed on imaging studies. Her diagnosis was hypertensive intracerebral hemorrhage and was treated conservatively. She developed subarachnoid hemorrhage 4 months later, and imaging studies detected dural arteriovenous fistula at the left C1 dural sleeve. Intracerebral hemorrhage and subarachnoid hemorrhage both occurred because of the disruption of a distended cerebellar vein due to venous hypertension caused by the dural arteriovenous fistula. Surgical ligation of a feeding artery and a draining vein was performed which cured the dural arteriovenous fistula [19].

\subsection{Case 3}

A report of delayed and progressive spontaneous closure of a DAVF after massive intracerebral hemorrhage documented by angiographic studies before and after bleeding. This is the first report to document gradual closure of a DAVF by serial angiographic studies. A 73-year-old woman who had an attack of generalized epileptic seizure was referred to a local hospital. Investigations like MRI and transfemoral cerebral angiography were done and revealed a DAVF Cognard type II $+\mathrm{b}$ of the left transverse sinus with feeders from the left occipital artery, left pharyngeal artery, left middle meningeal artery, and branches of the meningohypophyseal artery. There was reflux into the cerebral veins and the ipsilateral sigmoid sinus was not filled. She had conservative treatment and was discharged home on antiepileptic medications 3 days after admission. Also, she was planned a future endovascular therapy. She was admitted 1 week later after an episode of severe headache. Her neurological examination showed that her pupils were asymmetric and she was somnolent. A large and disseminated intracerebral hemorrhage in the left temporal, occipital, and parietal lobes was revealed on CT that was done upon admission. Her hematoma was evacuated surgically and she went through 
decompressive craniotomy 2 days later to treat her generalized brain edema and increased intracerebral pressure refractory to conservative therapy. The patient was sent for rehabilitation for hemiparesis and her recovery was slow. The DAVF closed completely 8 weeks after the hemorrhage. One year after the bleeding the patient was followed up, she was independent and had recovered but with mild aphasia and a slight hemiparesis on the right side [20].

\section{Conclusion}

Much has been learned in recent years about the pathoanatomy, pathophysiology, natural history, and therapeutic options for DAVFs. A better understanding of these lesions has allowed more prompt and precise diagnosis, in addition, a realistic assessment of features predisposing to aggressive clinical course. Clinical symptoms other than hemorrhage and progressive neurological deficits rarely warrants aggressive treatment of a DAVF, unless the lesion is particularly accessible or is associated with features predisposing to subsequent aggressive clinical behavior. Patient reassurance, symptomatic treatment, or palliative therapy is frequently sufficient. In DAVFs with features predisposing to an aggressive clinical course, a more definitive treatment strategy should be adopted. It is obvious that the myriad of clinical manifestations of DAVFs and the wide spectrum of possible angiographic and pathophysiologic scenarios call for highly individualized management strategies. The diagnostic investigation should be thorough to identify DAVFs with features predisposing to aggressive clinical behavior such as retrograde and leptomeningeal CVD, associated aneurysms, or venous ectasia.

\section{Conflict of interest}

There are no conflicts of interest to declare.

\section{Author details}

Sara A. AbuSini, Nour A. Tashtush, Abdelwahab J. Aleshawi and Amer A. Jaradat* Faculty of Medicine, Jordan University of Science and Technology, Irbid, Jordan

*Address all correspondence to: amerakramjaradat@yahoo.com

IntechOpen

(C) 2019 The Author(s). Licensee IntechOpen. This chapter is distributed under the terms of the Creative Commons Attribution License (http://creativecommons.org/licenses/ by/3.0), which permits unrestricted use, distribution, and reproduction in any medium, provided the original work is properly cited. (cc) BY 


\section{References}

[1] Jafar J, Awad I, Huang P. Intracranial vascular malformations: Clinical decisions and multimodality management strategies. In: Jafar J, Awad I, Rosenwasser R, editors. Vascular Malformations of the Central Nervous System. Philadelphia: Lippincott Williams \& Wilkins; 1999. pp. 219-232

[2] Awad I. The diagnosis and management of intracranial dural arteriovenous malformations. Contemporary Neurosurgery. 1991;13:1-6

[3] Awad I. Dural arteriovenous malformations. In: Carter L, Spetzler R, editors. Neurovascular Surgery. New York: McGraw-Hill; 1994. pp. 905-932

[4] Soderman M, Pavic L, Edner G, Holmin S, Andersson T. Natural history of dural arteriovenous shunts. Stroke. 2008;39:1735-1739

[5] Davies MA, Saleh J, Ter Brugge K, Willinsky R, Wallace MC. The natural history and management of intracranial dural arteriovenous fistulae: Part 1: Benign lesions. Interventional Neuroradiology. 1997;3:295-302

[6] Davies MA, Ter Brugge K, Willinsky R, Wallace MC. The natural history and management of intracranial dural arteriovenous fistulae: Part 2: Aggressive lesions. Interventional Neuroradiology. 1997;3:303-311

[7] HalbachVV,HigashidaRT,HieshimaGB, Mehninger CM, Hardin CW. Transvenous embolization of dural fistulas involving the transverse and sigmoid sinuses. AJNR. American Journal of Neuroradiology. 1989;10:385-392

[8] Newton TH, Cronqvist S. Involvement of dural arteries in intracranial arteriovenous malformations. Radiology. 1969;93:1071-1078

[9] Luciani A, Houdart E, Mounayer C, Saint MauriceJP, MerlandJJ. Spontaneous closure of dural arteriovenous fistulas: Report of three cases and review of the literature. AJNR. American Journal of Neuroradiology. 2001;22:992-996

[10] Borden JA, Wu JK, Shucart WA. A proposed classification for spinal and cranial dural arteriovenous fistulous malformations and implications for treatment. Journal of Neurosurgery. 1995;82:166-179

[11] Elhammady MS, Ambekar S, Heros RC. Epidemiology, clinical presentation, diagnostic evaluation and prognosis of cerebral dural arteriovenous fistulas. Handbook of Clinical Neurology. 2017;143:99-105

[12] Miller TR, Gandhi D. Intracranial dural arteriovenous fistulae clinical presentation and management strategies. Stroke. 2015;46:2017-2025

[13] Tsai LK, Liu HM, Jeng JS. Diagnosis and management of intracranial dural arteriovenous fistulas. Expert Review of Neurotherapeutics. 2016;16:307-318

[14] Chaichana KL, Coon AL, Tamargo RJ, Huang J. Dural arteriovenous fistulas: Epidemiology and clinical presentation. Neurosurgery Clinics of North America. 2012;23:7-13

[15] Gandhi D, Chen J, Pearl M, Huang J, Gemmete JJ, Kathuria S. Intracranial dural arteriovenous fistulas: Classification, imaging findings, and treatment. AJNR. American Journal of Neuroradiology. 2012;33:1007-1013

[16] Serulle Y, Miller TR, Gandhi D. Dural arteriovenous fistulae imaging and management. Neuroimaging Clinics of North America. 2016;26:247-258 
[17] Gupta A, Periakaruppan A.

Intracranial dural arteriovenous fistulas:

A review. Indian Journal of Radiology

and Imaging. 2009;19:43-48

[18] Amuluru K, Al-Mufti F, Romero C.

Isolated intraventricular hemorrhage

secondary to dural arteriovenous fistula.

Journal of NeuroInterventional Surgery.

2018;10:e7

[19] Inamasu J, Tanaka R, Nakahara I, Hirose Y. Dural arteriovenous fistula of the craniocervical junction

manifesting as cerebellar hemorrhage. The Neuroradiology Journal.

2016;29:356-360

[20] Al-Afif S, Nakamura M, Götz F, Krauss JK. Spontaneous closure of a dural arteriovenous fistula. BML Case Reports. 2014. pii: bcr2014011255 
Section 5

Moyamoya Disease 



\title{
Chapter 8
}

\section{Moyamoya Disease: A Rare Vascular Disease of the CNS}

\author{
Abdulgafoor M. Tharayil, Adel E. Ahmed Ganaw, \\ Nissar Shaikh, Sujith M. Prabhakaran, Arshad H. Chanda, \\ Simi Praveen, Ajith Kumar Choran and Qazi Zeeshan ul Haq
}

\begin{abstract}
Moyamoya disease (MMD) is a rare disease affecting the cerebral vasculature of the central nervous system (CNS) with a reported incidence of 0.35-0.94 per 100,000 populations. It was first reported from Japan and later from other parts of the world. The pathology is narrowing of blood vessels supplying anterior circulation and rarely posterior circulation. It was believed that the disease is genetic in origin, but environmental factors also play a role. Patients with this rare disease may present with ischemic or hemorrhagic symptoms. Ischemic symptoms account for the disease in most of the pediatric patients, whereas in adults, hemorrhage is more common. Diagnostic imaging like CT angiogram and magnetic resonance angiogram helps in demonstrating the narrowing or the collateral vessels like "a puff of smoke" (moyamoya) formed at the base of the brain. Moyamoya disease is treated medically and/or surgically. Aspirin is the main medication used. Surgical options are direct or indirect revascularization techniques to bypass the stenosis. The disease is progressive in majority of the patients, but if treated early, they can have good prognosis especially children.
\end{abstract}

Keywords: moyamoya, vascular, CNS, MMD, MMS

\section{Introduction}

Moyamoya disease (MMD) is a rare disease of the central nervous system (CNS). It was first reported in Japan in the year 1957 and later reported from other Asian countries. "Moyamoya" in Japanese means "a puff of smoke" alluding to the characteristic angiographic appearance of the collateral circulation in the brain of the patient with moyamoya disease [1]. It can present either as an isolated condition (moyamoya disease) or as a part of a syndrome associated with other conditions. In the latter case, it is called moyamoya syndrome (MMS). Moyamoya disease usually manifests bilaterally [2], and moyamoya syndrome manifests, unilaterally, to begin with, which may progress to become bilateral disease [3].

\section{Etiology}

Ten to fifteen percent of moyamoya disease (MMD) is familial in origin which indicates a genetic association. East Asian populations with RNF213 gene on chromosome 
17q25.3 are susceptible to MMD [4]. In another report from Japan, it was observed that a variant of RNF213 (c.14576G) was present in 41 patients with familial MMD (95\%), 163 patients with sporadic MMD (79\%), and 283 normal control subjects (2\%) [5]. In a study in Chinese Han population, Wu et al. demonstrated that mutations in RNF213 gene were associated with increased susceptibility to MMD. In further analysis, they observed that ischemic MMD was related to the R4810K mutation, and hemorrhagic MMD was associated with the A4399T mutation [6]. Mineharu et al. suggested that MMD is an autosomal dominant disease with incomplete penetrance [7]. Inoue et al. observed that different alleles of genes of HLA antigen have been found to be associated with MMD [8]. Several inducers of angiogenesis such as fibroblast growth factor, transforming growth factor beta1, and hepatocyte growth factor which promote neovascularization were found in high levels in patients with MMD [9-11].

Moyamoya syndrome (MMS) is a different entity when the disease is associated with some other conditions such as [12]:

\section{Atherosclerosis}

2. Infectious diseases: meningitis and other viral or bacterial infections

3. Hematologic conditions: sickle cell disease, beta thalassemia, Fanconi anemia, hereditary spherocytosis, homocystinuria and hyper-homocysteinemia, factor XII deficiency, essential thrombocythemia

4. Vasculitis and autoimmune diseases: systemic lupus erythematosus, polyarteritis nodosa postinfectious vasculopathy, Graves' disease thyroiditis, Sneddon syndrome, antiphospholipid antibody syndrome, anti-Ro and anti-La antibodies

\section{Type 1 diabetes mellitus}

6. Connective tissue disorders and neurocutaneous syndromes: neurofibromatosis type 1 (NF1), tuberous sclerosis, Sturge-Weber syndrome, phakomatosis pigmentovascularis type IIIb, hypomelanosis of Ito, pseudoxanthoma elasticum, Marfan syndrome, chromosomal disorders

7. Chromosomal disorders: down syndrome, turner syndrome, Alagille syndrome

8. Other vasculopathies: vasospasm after subarachnoid hemorrhage, radiation therapy to the base of the brain, fibromuscular dysplasia

9. Other extracranial cardiovascular diseases: congenital heart disease, Williams syndrome, coarctation of the aorta, renal artery stenosis

10. Metabolic diseases: type I glycogenosis, hyperphosphatasia, primary oxalosis

11. Cranial trauma

12. Brain tumors

13. Cavernous malformation

14. Pulmonary sarcoidosis 
15. Hereditary multisystem disorder with short stature, hypergonadotropic hypogonadism, and dysmorphism

\section{Polycystic kidney disease}

There are reports of identical twins with only one among them affected by MMD, which question the genetic basis and focus on environmental factors for the condition [13].

\section{Epidemiology}

Although originally reported in the Asian population, moyamoya disease has also been reported from Europe and America [1].

In an epidemiological study by Baba et al. [14], MMD was found to have the following characteristics in the Japanese population:

- Annual incidence of $0.35-0.94$ per 100,000 populations

- A prevalence of $3.2-10.5$ per 100,000 populations

- A male-to-female ratio of 1:1.8-1:2.2 reflecting a female predilection

- A family history of MMS in $10.0-15.4 \%$ of patients

Although the reported incidence of MMS from Washington and California was as low as 0.086 per 100,000 populations generally, in ethnic groups, it was found to be as high as 0.28 per 10,000 populations close to the incidence in Japan [15].

MMS shows a bimodal distribution in the Chinese population with a major peak in the 5-9-year-old group and another peak in the 35-39-year-old group [16]. In the Japanese population, there are two conflicting reports of higher peaks, one in childhood and another in adults [14, 17].

\section{Clinical presentation}

Patients affected by MMD present with ischemia like transient ischemic attack (TIA) and stroke and seizures or with intraparenchymal bleeds due to the rupture of fragile collateral vessels formed to compensate for the ischemia. They may also present with headache due to dilated transdural collateral blood vessels. A 2012 systematic review of population-based studies by Kleinloog et al. found that the predominant mode of presentation was ischemia, especially in children [18]. Ninety percent of children present with stroke and 7.5\% present with TIA as per International Pediatric Stroke Study published in 2017 [19]. In children, exercise, crying, coughing, straining, fever, or hyperventilation can trigger symptomatic episodes of ischemia.

Adult patients in Japan with MMS mostly present with hemorrhagic stroke in some old reports [20] in contrast to patients from the United States in whom the ischemic stroke was found to be more common [21]. In a study done on 88 Korean patients, $45 \%$ of them had only a single ischemic or hemorrhagic stroke, $55 \%$ recurrent attacks mostly ischemic, and $64 \%$ of them presented with hemiplegia [22].

Ischemic symptoms of MMD are typically associated with the anterior circulation regions like frontal, parietal, and temporal lobes supplied by internal carotid 
(ICA) and middle cerebral artery (MCA). Commonly the symptoms are hemiparesis, dysarthria, aphasia, and cognitive impairment [23]. Some patients may present with seizures, defective vision, syncopal attacks, or personality changes and can mimic psychiatric disorders [24]. Surgical patients undergoing minor procedures under anesthesia may present with perioperative stroke due to hyperventilationinduced cerebral vasoconstriction of the already compromised cerebral vasculature of the ischemic penumbra [25]. Patients with MMD can develop cerebral aneurysms at the tip of the basilar artery and the posterior communicating artery and present with subarachnoid hemorrhage [26].

Choreiform movements are another group of symptoms with which children can present due to the moyamoya-associated dilated collateral vessels in the basal ganglia [27]. Occasionally a characteristic ophthalmologic finding is seen in association with MMD identified as "morning glory disk," an optic disk enlargement with concomitant retinovascular anomalies [28].

\section{Pathophysiology}

In patients with this condition, large intracerebral arteries show a variable degree of stenosis and occlusions. Vascular intima shows fibrocellular thickening; elastic internal lamina will be tortuous, duplicate or triplicate; and the media show attenuation. Vessels may be dilated and thin-walled or thick-walled and stenosed in different regions. The hallmark of MMD is the meshwork of dilated vessels called moyamoya vessels (Figure 1). Aneurysms can form in some parts of cerebral vasculature as well.

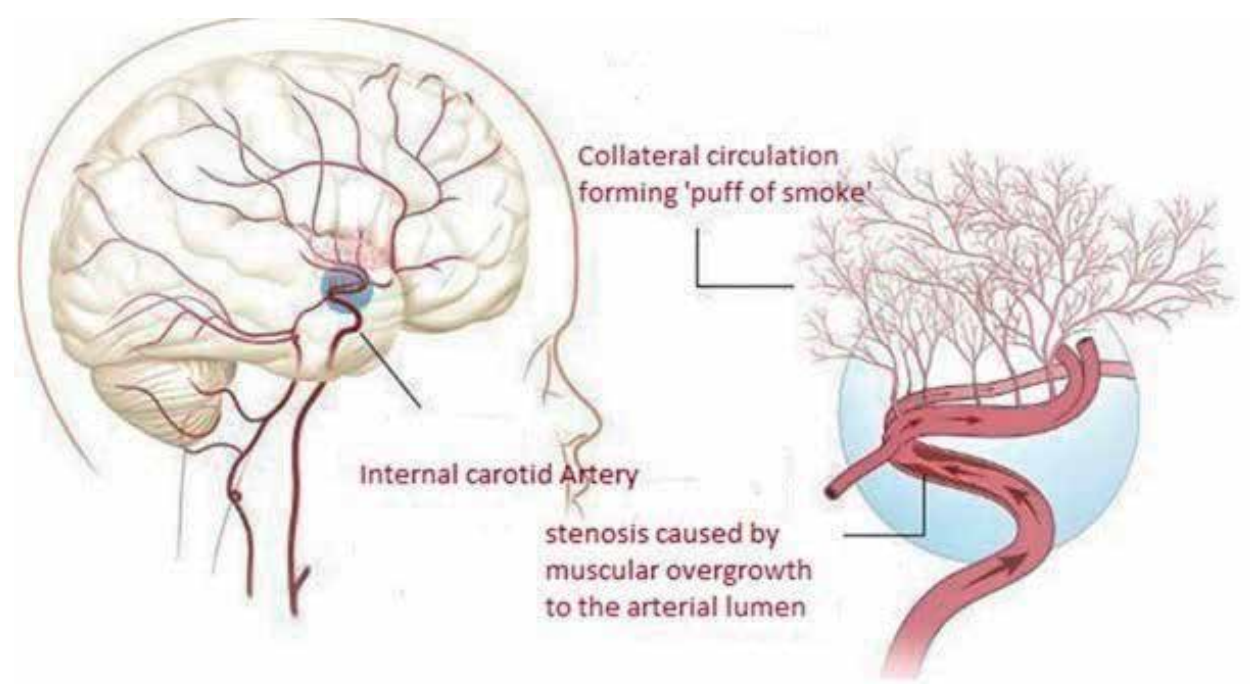

Figure 1.

Pathophysiology of moyamoya disease (modified from [29]).

\section{Diagnostic radiology}

Diagnosis can be established by invasive and semi-invasive radiological studies. At the bedside, transcranial Doppler (TCD) can detect stenosis of major arteries. Conventional CT or MRI helps to detect ischemic and hemorrhagic signs of MMD. CT angiography and conventional angiography are important to establish the exact point of narrowing in the circle of Willis. 


\begin{tabular}{ll}
\hline Stage & Characteristics \\
\hline Stage 1 & - Narrowing of carotid fork only \\
& - Narrowed ICA bifurcation \\
\hline Stage 2 & - Initiation of basal moyamoya \\
& - Dilated ACA, MCA, and narrowed ICA bifurcation with moyamoya change \\
\hline Stage 3 & - Intensification of the "moyamoya" \\
& - Further increase in moyamoya change of the ICA bifurcation and narrowed ACA and MCA \\
& together with the reduction of flow \\
\hline Stage 4 & - Minimization of moyamoya vessels \\
& - Moyamoya change reducing with occlusive changes in ICA and tenuous ACA and MCA \\
\hline Stage 5 & Reduction of moyamoya \\
& - Further decrease in moyamoya change with occlusion of ICA, ACA, and MCA (no flow) \\
\hline Stage 6 & Disappearance of moyamoya vessels \\
& The cerebral circulation is supplied only by the external carotid system
\end{tabular}

Table 1.

Suzuki staging system for moyamoya.

Suzuki and coworkers studied the angiographic progression of MMD and identified six stages (Table 1) of progression $[1,2]$.

This staging neither correlates with disease severity nor allows therapeutic risk stratification.

\subsection{CT head}

Cortical and/or subcortical infarction can be seen in the early stages, but in the later stages, dilatation of sulci or ventricles due to loss of volume can be seen. In a retrospective case series of 32 patients by Kim et al. [30], early stages (stages 1 and 2 of MMD) had mainly subcortical ischemic changes, whereas later stages (stage 3 and above) had cortical ischemic changes.

\subsection{Magnetic resonance imaging}

Diffusion and perfusion MR techniques are superior to CT scan for detection of subtle ischemic brain lesions especially in the acute condition. MRI was sensitive to detect watershed infarction in $50 \%$ of children as per a study done in Canada [31]. Dilated collateral vessels at the base of the brain are considered pathognomonic of MMD but seen only in some cases. In T2 sequences asymptomatic microbleeds may be seen. FLAIR images and T1 sequences may show a characteristic sign called ivy sign as in Figure 2 (a linear pattern of increased signal in the leptomeninges and perivascular spaces) [33]. This is due to retrograde flow in leptomeninges resembling ivy creeping a stone. Magnetic resonance angiography (MRA) has surpassed conventional angiography in some centers as the primary imaging method although the latter is still considered as the gold standard.

\subsection{Angiography}

Conventional cerebral angiography is the gold standard for the diagnosis of MMD as it can clearly demonstrate the stenotic vessels in the anterior circulation as well as the collateral tuft of vessels formed (Figure 3). Apart from this, the small aneurysms which may be associated with MMD also can be detected in cerebral angiography. 


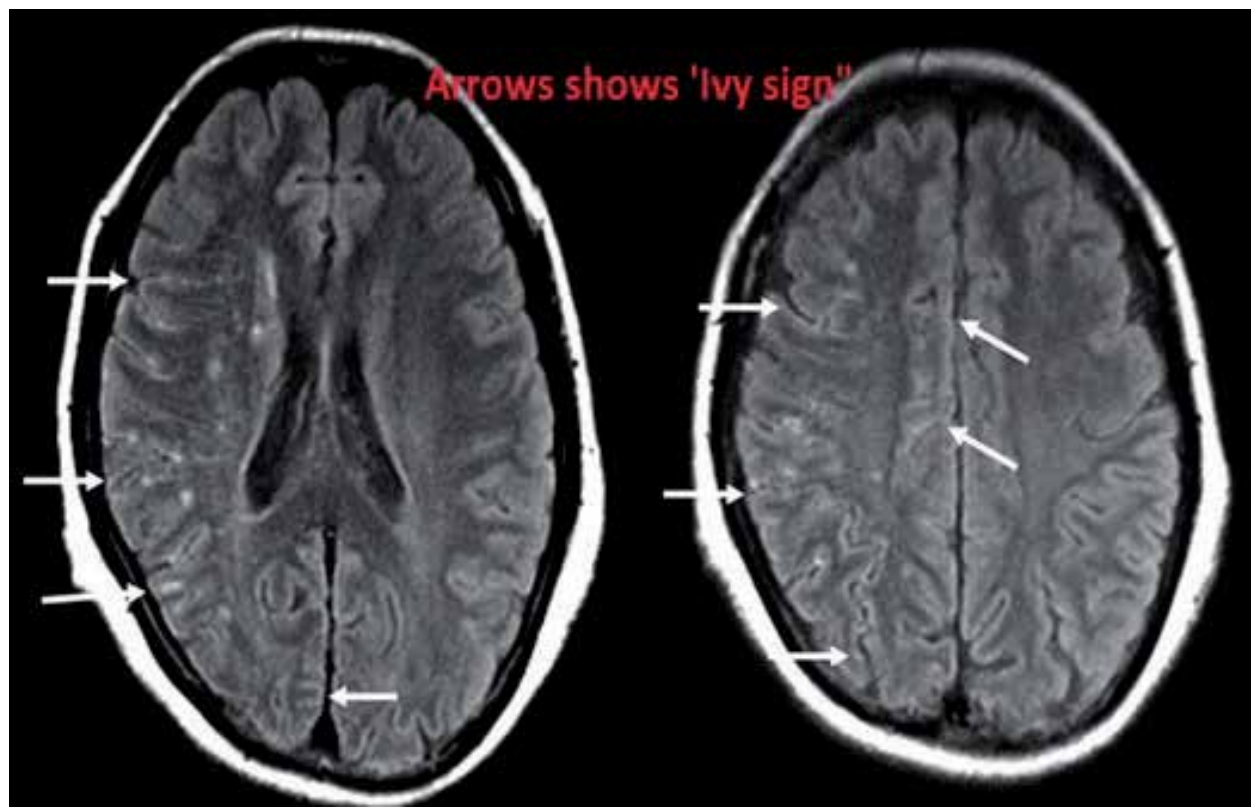

Figure 2.

FLAIR sequence showing ivy sign (modified from [32]).

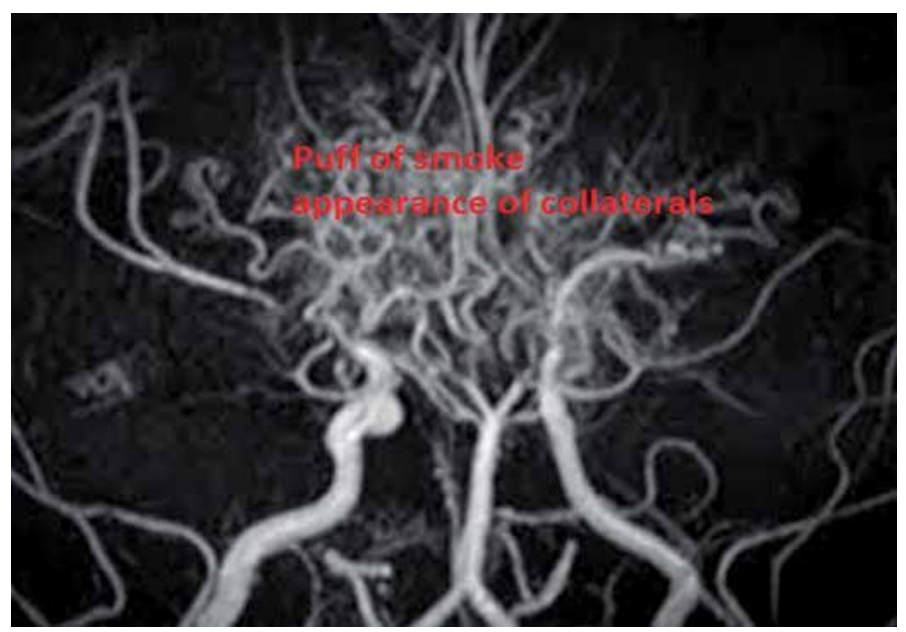

Figure 3.

Characteristic "puff of smoke" appearance in cerebral angiography.

\subsection{Transcranial Doppler (TCD)}

Stenosed vessels will have increased flow velocity proportional to the degree of stenosis, which can be detected noninvasively by TCD.

\subsection{Electro encephalogram (EEG)}

A specific pattern of hyperventilation-induced diffuse monophasic slow waves called "build-up" and post-hyperventilation "rebuild-up" waves similar to build-up waves in patients without MMD may be observed in patients with MMD [34]. 
Perfusion CT, xenon-enhanced CT, perfusion-weighted MRI, positron emission tomography (PET), and single-photon emission CT (SPECT) with acetazolamide challenge are other modalities rarely used in diagnosis [12].

\section{Diagnostic criteria for MMD}

A Japanese research group has put forward the following diagnostic criteria for the diagnosis of MMD [35]:

- Stenosis or occlusion at the terminal portion of the internal carotid artery and at the proximal portion of the anterior and middle cerebral arteries on magnetic resonance angiography (MRA).

- Abnormal vascular networks in the basal ganglia on MRA; these networks can also be diagnosed by the presence of multiple flow voids on brain MRI.

- Bilateral angiographic findings; although unilateral angiographic findings are considered probable.

- Conditions to be excluded:

$\circ$ Arteriosclerosis

$\circ$ Autoimmune disease

$\circ$ Brain neoplasm

$\circ$ A history of cranial irradiation

$\circ$ Down syndrome

$\circ$ Head trauma

- Neurofibromatosis

$\circ$ Meningitis

\section{Treatment of MMD}

In the acute stage, treatment is symptomatic for those patients with ischemic or hemorrhagic stroke as per acceptable guidelines. It should be focused on treating elevated intracranial pressure, evacuation of hematoma, draining intraventricular hemorrhage by external ventricular drain (EVD), control of seizures, glycemic control, and treatment of fever.

In children diagnosed with MMD, crying and hyperventilation should be avoided as it can induce cerebral vasoconstriction. Pain management also should be taken care of. Supplemental oxygen also helps in avoiding hypoxia. Generally, hypotension, hyperthermia, hypoxia, hypocarbia, and hypovolemia should be avoided.

There are no interventions proved to improve outcome in MMD. The utility of thrombolysis has not been studied yet and may be risky due to the possibility 
of development of hemorrhage from the fragile collateral vessels [36]. Aspirin has been recommended by the American College of Chest Physicians (ACCP) for children with ischemic stroke [37].

There were no significant differences in outcome between medically and surgically treated patients with MMD as per a large survey from Japan, but a later review revealed that $38 \%$ of 651 patients with MMD who initially underwent medical management ultimately had to go for surgery because of progressive symptoms [38].

\subsection{Secondary stroke prevention}

Treatment of underlying diseases like sickle cell disease is helpful in secondary prevention. Surgical revascularization is the mainstay of secondary prevention. Antiplatelet medications like aspirin have an important role in preventing stroke in mild disease or asymptomatic patients who are at high risk for surgery [39]. Oral anticoagulants are not helpful as they can increase the chance of hemorrhage. Calcium channel blockers were used in some reports [40]. Endovascular embolization has been used for the obliteration of aneurysm associated with MMD.

\section{Surgical management of moyamoya disease}

The aim of surgical management of moyamoya disease is to vascularize and restore the blood supply by bypassing the stenosed area. It will stabilize the cerebrovascular hemodynamics. It prevents bleeding by preventing the progression of the thin moyamoya vessels. Improvement and or normalization of cerebral hemodynamics by the surgical intervention will also prevent secondary stroke prevention and improve the neurological and neurocognitive outcome.

Revascularization surgery is the most effective treatment for hemorrhagic MMD [41] and can be effective in preventing future stroke events in adults [42].

The main indications for the surgical interventions are cerebral hemodynamic impairment and repeat ischemic symptoms. The severity of the disease to assign a patient for surgical intervention can be made by the Berlin moyamoya grading system (Table 2). But the literature has not mentioned the severity at which there is an indication for surgical intervention.

\begin{tabular}{lcc}
\hline Variable & Characteristics & Points \\
\hline $\begin{array}{l}\text { Digital subtraction } \\
\text { angiography }\end{array}$ & Steno-occlusive lesion + moyamoya vessels & 1 \\
\cline { 2 - 3 } & $\begin{array}{c}\text { Steno-occlusive lesion + moyamoya } \\
\text { vessels + intracranial compensation routes }\end{array}$ & 2 \\
\cline { 2 - 3 } & Steno-occlusive lesion + extracranial compensation \\
routes & 3 \\
\hline MRI & No signs of ischemia, hemorrhage, or atrophy & 0 \\
\hline Cerebrovascular reserve & Signs of ischemia, hemorrhage, or atrophy & 1 \\
\cline { 2 - 3 } capacity & No steal phenomenon $(\geq 5 \%)$ & 0 \\
\hline
\end{tabular}

Table 2.

The Berlin moyamoya grading [43]. 


\section{The Berlin moyamoya grading}

It incorporates digital subtraction angiography (DSA), MRI, and cerebrovascular reserve capacity (CVRC) which gives the information of the functional cerebrovascular assessment of hemodynamic impairment [43].

It was proposed by Czabanka et al. According to the grading system, there are three grades:

1. Grades 1-2: mild

2. Grades 3-4: moderate

3. Grades 5-6: severe

\section{Indications for surgical revascularization}

Patients with ischemic symptoms and progressive disease with impairment in cognition benefit from surgical revascularization methods. It can be achieved by direct and indirect methods.

\subsection{Direct surgical treatment}

A branch of the external carotid artery is connected to the branch of the internal carotid artery beyond the stenosis to bypass the stenosed segment. Superficial temporal artery to middle cerebral artery (STA-MCA) (Figure 4) bypass is the commonly used technique [45]. Other less common direct techniques are STA-ACA, STA-PCA, and occipital artery-PCA anastomosis. These direct techniques are used when an immediate recirculation is indicated. The major drawback is that this cannot be easily done in children with MMD because of the smaller size of donor and recipient arteries.

\subsection{Indirect surgical treatment}

In this technique, a vascularized tissue supplied by the external carotid artery (e.g., dura, temporalis muscle, or the superficial temporal artery itself) is placed in direct contact with the brain surface, which results in ingrowth of new blood vessels to the underlying cerebral cortex (Figure 5). In this technique, good cerebral blood flow is established after a few weeks and so not suitable for emergency cases [46], but good for pediatric patients with MMD because of the lower technical difficulty. The following are the options for indirect revascularization [47]:

- Encephaloduroarteriosynangiosis (EDAS) and its modification called pial synangiosis

- Encephalomyosynangiosis (EMS)

- Encephalo-duro-arterio-myosynangiosis (EDAMS)

- Encephaloarteriosynangiosis

- Encephaloduroarteriosynangiosis

- Omental transplantation 
- Craniotomy with inversion of the dura

- Multiple burr holes without vessel synangiosis

- Cervical sympathectomy

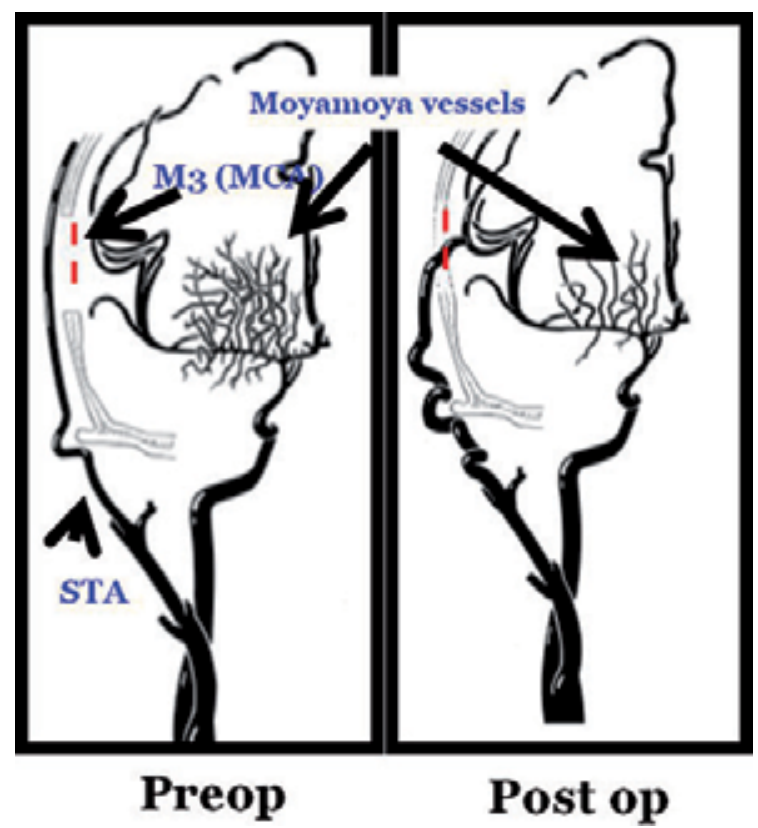

Figure 4.

Direct bypass procedure of superficial temporal artery (STA) and $M_{3}$ branches of the middle cerebral artery (MCA) (modified from Acker et al. [44]).

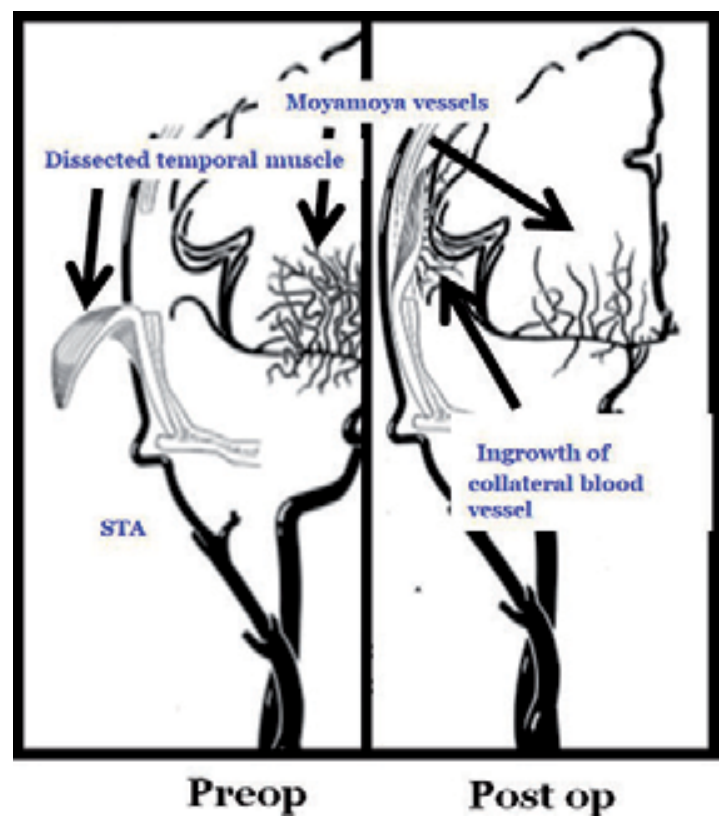

Figure 5.

Indirect bypass procedure of indirect bypass procedure: dissected temporal muscle on the brain surface (modified from Acker et al. [44]). 


\subsection{Combined techniques}

Houkin et al. first performed a combined revascularization surgery. It combined the superficial temporal artery and M3 branches of the middle cerebral artery (STA-MCA) bypass and encephalo-duro-arterio-myosynangiosis (EDAMS) (Figure 6). The aim of the combined procedure is to improve the results to serve as an alternative in case the direct bypass fails and prevents second surgery. The direct bypass immediately improves the hemodynamics [44]. A 2005 systematic review from Japan compared direct, indirect, and combined techniques and found that there is no statistically significant difference between all these techniques in terms of outcome during 58 months of follow-up period [48]. Efficacy of medical and surgical management was also compared in a large survey conducted in Japan and concluded that there is no significant difference between the two [49]. However, in another study, it was found that children initially underwent medical therapy had to ultimately go for revascularization surgery more than the children who underwent surgery in the first instance [38].

\subsection{The complication of the surgical revascularization}

Surgical revascularization is not risk-free. This can range from a transient increase in intracranial pressure resulting in headache, seizures, and reversible neurological deficits to perioperative stroke. The mechanism is cerebral hyperperfusion syndrome because of the revascularization-induced hyperperfusion of the area supplied by the chronically vasoconstricted blood vessels, where the autoregulation is impaired. Incidence of this phenomenon was found to be up to $47 \%$ [50].

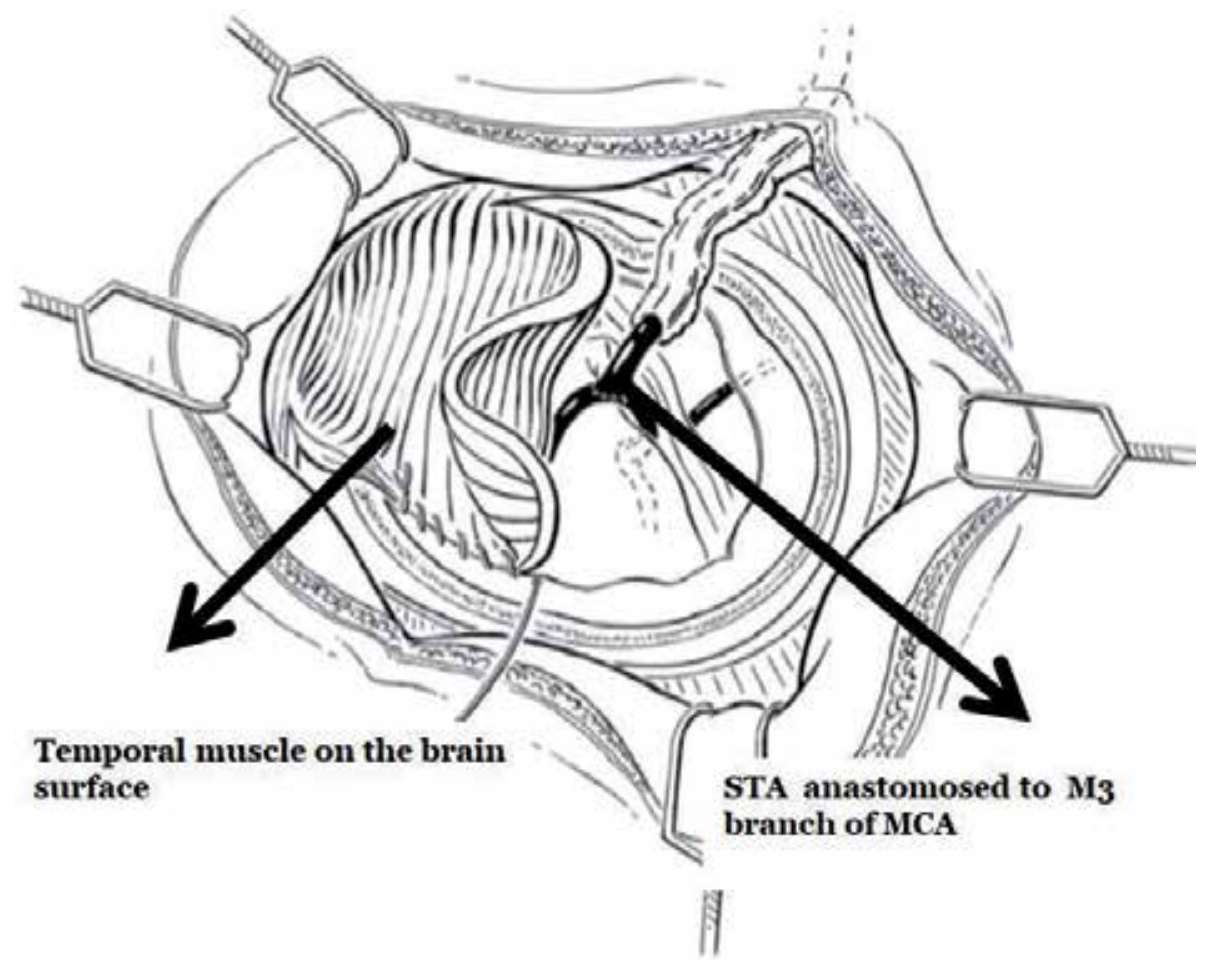

Figure 6.

Combined revascularization procedure. The temporal muscle is positioned on the brain surface, and the STA is a bypass graft to the M3 branch of MCA (modified from Acker et al. [44]). 
All adults and children with ischemic MMD or MMS should have aspirin for a long term. If there are no contraindication and significant ischemic symptoms, surgical revascularization is a reasonable option [47]. AHA guidelines recommend it for patients with compromised cerebrovascular perfusion according to blood flow studies [39]. As mentioned before there is no evidence for opting for direct or indirect revascularization or vice versa, though the latter is preferred in the pediatric age group. Evidence for postoperative use of aspirin is limited. In a recent retrospective study, patients treated with postoperative aspirin had better outcome $[44,51]$.

\section{Prognosis}

Prognosis depends on how fast the blockage occurs and how effective the collateral circulation is established. Fifty to sixty-six percent of patients will have the progression of the disease, and they have a poor outcome. Pediatric patients who were surgically treated were found to have disease progression in an estimated $2.6 \%$ of patients only. Among untreated asymptomatic patients, the annual stroke rate was found to be $3.2 \%$ and disease progression in $80 \%[52,53]$.

\section{Author details}

Abdulgafoor M. Tharayil ${ }^{1,2 *}$, Adel E. Ahmed Ganaw ${ }^{2}$, Nissar Shaikh ${ }^{1,2}$, Sujith M. Prabhakaran ${ }^{2}$, Arshad H. Chanda ${ }^{2}$, Simi Praveen ${ }^{2}$, Ajith Kumar Choran ${ }^{2}$ and Qazi Zeeshan ul Haq ${ }^{2}$

1 Weill-Cornell Medicine, Qatar

2 Hamad Medical Corporation, Qatar

*Address all correspondence to: atharayil@hamad.qa

IntechOpen

(C) 2019 The Author(s). Licensee IntechOpen. This chapter is distributed under the terms of the Creative Commons Attribution License (http://creativecommons.org/licenses/ by/3.0), which permits unrestricted use, distribution, and reproduction in any medium, provided the original work is properly cited. (cc) BY 


\section{References}

[1] Suzuki J, Kodama N. Moyamoya disease-A review. Stroke. 1983;14:104-109

[2] Suzuki J, Takaku A. Cerebrovascular "moyamoya" disease: Disease showing abnormal net-like vessels in base of brain. Archives of Neurology. 1969;20:288-299

[3] Fukui M. Guidelines for the diagnosis and treatment of spontaneous occlusion of the circle of Willis ('moyamoya' disease). Clinical Neurology and Neurosurgery. 1997;99(Suppl 2): S238-S240

[4] Miyawaki S, Imai H, Takayanagi S, et al. Identification of a genetic variant common to moyamoya disease and intracranial major artery stenosis/ occlusion. Stroke. 2012;43:3371.9

[5] Miyatake S, Miyake N, Touho H, et al. Homozygous c. $14576 \mathrm{G}>\mathrm{A}$ variant of RNF213 predicts early-onset and severe form of moyamoya disease. Neurology. 2012;78:803

[6] Wu Z, Jiang H, Zhang L, et al. Molecular analysis of RNF213 gene for moyamoya disease in the Chinese Han population. PLoS ONE. 2012;7:e48179

[7] Mineharu Y, Takenaka K, Yamakawa $\mathrm{H}$, et al. Inheritance pattern of familial moyamoya disease: Autosomal dominant mode and genomic imprinting. Journal of Neurology, Neurosurgery, and Psychiatry. 2006;77:1025

[8] Inoue TK, Ikezaki K, Sasazuki T, et al. Analysis of class II genes of human leukocyte antigen in patients with moyamoya disease. Clinical Neurology and Neurosurgery. 1997;99(Suppl 2):S234

[9] Yamamoto M, Aoyagi M, Tajima S, et al. Increase in elastin gene expression and protein synthesis in arterial smooth muscle cells derived from patients with Moyamoya disease. Stroke. 1997;28:1733

[10] Hojo M, Hoshimaru M, Miyamoto S, et al. Role of transforming growth factor-beta 1 in the pathogenesis of moyamoya disease. Journal of Neurosurgery. 1998;89:623

[11] Nanba R, Kuroda S, Ishikawa T, et al. Increased expression of hepatocyte growth factor in cerebrospinal fluid and intracranial artery in moyamoya disease. Stroke. 2004;35:2837

[12] Suwanwela NC. Moyamoya Disease: Etiology, Clinical Features, and Diagnosis. Post TW, editor, UpToDate. Waltham, MA: UpToDate Inc. Available from: https://www.uptodate.com [Accessed: 28 May 2019]

[13] Tanghetti B, Capra R, Giunta F, Marini G, Orlandini A. Moyamoya syndrome in only one of two identical twins: Case report. Journal of Neurosurgery. 1983;59:1092-1094

[14] Baba T, Houkin K, Kuroda S. Novel epidemiological features of moyamoya disease. Journal of Neurology, Neurosurgery, and Psychiatry. 2008;79:900

[15] Uchino K, Johnston SC, Becker KJ, Tirschwell DL. Moyamoya disease in Washington state and California. Neurology. 2005;65:956

[16] Duan L, Bao XY, Yang WZ, et al. Moyamoya disease in China: Its clinical features and outcomes. Stroke. 2012;43:56

[17] Wakai K, Tamakoshi A, Ikezaki K, et al. Epidemiological features of moyamoya disease in Japan: Findings from a nationwide survey. Clinical Neurology and Neurosurgery. 1997;99(Suppl 2):S1 
[18] Kleinloog R, Regli L, Rinkel GJ, Klijn CJ. Regional differences in incidence and patient characteristics of moyamoya disease: A systematic review. Journal of Neurology, Neurosurgery, and Psychiatry. 2012;83:531

[19] Lee S, Rivkin MJ, Kirton A, et al. Moyamoya disease in children: Results from the international pediatric stroke study. Journal of Child Neurology. 2017;32:924

[20] Kitamura K, Fukui M, Oka K. Moyamoya disease. In: Handbook of Clinical Neurology. Vol. 2. Amsterdam: Elsevier; 1989. p. 293

[21] Zafar SF, Bershad EM, Gildersleeve KL, et al. Adult moyamoya disease in an urban center in the United States is associated with a high burden of watershed ischemia. Journal of the American Heart Association. Aug 2014;3(4):e001123

[22] Choi JU, Kim DS, Kim EY, Lee KC. Natural history of moyamoya disease: Comparison of activity of daily living in surgery and nonsurgery groups. Clinical Neurology and Neurosurgery. 1997;99(Suppl 2):S11

[23] Scott RM, Smith JL, Robertson RL, Madsen JR, Soriano SG, Rockoff MA. Long-term outcome in children with moyamoya syndrome after cranial revascularization by pial synangiosis. Journal of Neurosurgery. 2004;100(Suppl):142-149

[24] Lubman DI, Pantelis C, Desmond P, Proffitt TM, Velakoulis D. Moyamoya disease in a patient with schizophrenia. Journal of the International Neuropsychological Society. 2003;9:806-810

[25] Tagawa T, Naritomi H, Mimaki T, Yabuuchi H, Sawada T. Regional cerebral blood flow, clinical manifestations, and age in children with moyamoya disease. Stroke. 1987;18:906-910
[26] Kawaguchi S, Sakaki T, Morimoto T, Kakizaki T, Kamada K. Characteristics of intracranial aneurysms associated with moyamoya disease: A review of 111 cases. Acta Neurochirurgica. 1996;138:1287-1294

[27] Parmar RC, Bavdekar SB, Muranjan MN, Limaye U. Chorea: An unusual presenting feature in pediatric moyamoya disease. Indian Pediatrics. 2000;37:1005-1009

[28] Massaro M, Thorarensen O, Liu GT, Maguire AM, Zimmerman RA, Brodsky MC. Morning glory disc anomaly and moyamoya vessels. Archives of Ophthalmology. 1998;116:253-254

[29] Available from: http:// strokeconnection.strokeassociation.org/ Fall-2014/Understanding-MoyamoyaDisease-in-Children/

[30] Kim JM, Lee SH, Roh JK. Changing ischaemic lesion patterns in adult moyamoya disease. Journal of Neurology, Neurosurgery, and Psychiatry. 2009;80:36

[31] Rafay MF, Armstrong D, Dirks P, et al. Patterns of cerebral ischemia in children with moyamoya. Pediatric Neurology. 2015;52:65

[32] Mori N, Mugikura S, Higano S, Kaneta T, Fujimura M, Umetsu A, et al. The leptomeningeal "ivy sign" on fluid-attenuated inversion recovery $\mathrm{mr}$ imaging in Moyamoya disease: A sign of decreased cerebral vascular reserve? American Journal of Neuroradiology. 2009;30(5):930-935

[33] Ohta T, Tanaka H, Kuroiwa T. Diffuse leptomeningeal enhancement, "ivy sign," in magnetic resonance images of moyamoya disease in childhood: Case report. Neurosurgery. 1995;37:1009

[34] Kodama N, AokiY, Hiraga H, Wada T, Suzuki J. Electroencephalographic 
findings in children with moyamoya disease. Archives of Neurology. 1979;36:16-19

[35] Fukui M. Guidelines for the diagnosis and treatment of spontaneous occlusion of the circle of Willis ('moyamoya' disease). Research committee on spontaneous occlusion of the circle of Willis (moyamoya disease) of the Ministry of Health and Welfare, Japan. Clinical Neurology and Neurosurgery. 1997;99(Suppl 2):S238

[36] Pollak L. Moyamoya disease and moyamoya syndrome. The New England Journal of Medicine. 2009;361:98. author reply 98

[37] Monagle P, Chan AK, Goldenberg NA, et al. Antithrombotic therapy in neonates and children: Antithrombotic therapy and prevention of thrombosis, 9th ed: American College of Chest Physicians Evidence-Based Clinical Practice Guidelines. Chest. 2012;141:e737S

[38] Ikezaki K. Rational approach to treatment of moyamoya disease in childhood. Journal of Child Neurology. 2000;15:350-356

[39] Roach ES, Golomb MR, Adams R, et al. Management of stroke in infants and children: A scientific statement from a special writing Group of the American Heart Association Stroke Council and the council on cardiovascular disease in the young. Stroke. 2008;39:2644

[40] Hosain SA, Hughes JT, Forem SL, et al. Use of a calcium channel blocker (nicardipine $\mathrm{HCl}$ ) in the treatment of childhood moyamoya disease. Journal of Child Neurology. 1994;9:378

[41] Miyamoto S, Yoshimoto T, Hashimoto N, Okada Y, Tsuji I, Tominaga T, et al., Effects of extracranial-intracranial bypass for patients with hemorrhagic moyamoya disease: results of the Japan Adult Moyamoya Trial. JAM Trial Investigators. Stroke. 2014;45(5): 1415-1421

[42] Jeon J, Kim J, Cho W, Bang J, Son Y, Oh C. Meta-analysis of the surgical outcomes of symptomatic moyamoya disease in adults. Journal of Neurosurgery. 2018;128(3):793-799

[43] Czabanka M, Peña-Tapia P, Schubert GA, Heppner FL, Martus P, Horn P, et al. Proposal for a new grading of moyamoya disease in adult patients. Cerebrovascular Diseases. 2011;32:4150. DOI: $10.1159 / 000326077$

[44] Acker G, Fekonja L, Vajkoczy P. Surgical management of moyamoya disease. Stroke. 2018;49:476-482. DOI: 10.1161/strokeaha.117.018563

[45] Golby AJ, Marks MP, Thompson RC, Steinberg GK. Direct and combined revascularization in pediatric moyamoya disease. Neurosurgery. 1999;45:50

[46] Scott RM, Smith ER. Moyamoya disease and moyamoya syndrome. The New England Journal of Medicine. 2009;360:1226-1237

[47] Suwanwela NC. Moyamoya Disease: Treatment and Prognosis. Post TW, editor. UpToDate. Waltham, MA: UpToDate Inc. Available from: https:// www.uptodate.com [Accessed: 29 May 2019]

[48] Fung LW, Thompson D, Ganesan V. Revascularisation surgery for paediatric moyamoya: A review of the literature. Child's Nervous System. 2005;21:358

[49] Fukui M. Current state of study on moyamoya disease in Japan. Surgical Neurology. 1997;47:138

[50] Hwang JW, Yang HM, Lee H, et al. Predictive factors of symptomatic 
cerebral hyperperfusion after superficial temporal artery-middle cerebral artery anastomosis in adult patients with moyamoya disease. British Journal of Anaesthesia. 2013;110:773

[51] Zhao Y, Zhang Q, Zhang D, Zhao Y. The effect of aspirin in the postoperative management of adult ischemic moyamoya disease. World Neurosurgery. 2017;105:728-731. DOI: 10.1016/j.wneu.2017.06.057

[52] Guzman R, Lee M, Achrol A, et al. Clinical outcome after 450 revascularization procedures for moyamoya disease. Journal of Neurosurgery. Nov 2009;111(5):927-935

[53] Kuroda S, Houkin K. Moyamoya disease: Current concepts and future perspectives. Lancet Neurology.

2008;7:1056-1066 
Section 6

\section{Spinal Epidural Haematomas}





\title{
Chapter 9
}

\section{Spontaneous Spinal Epidural Haematomas}

\author{
David Kieser, Scheherezade Soltani, Michael Wyatt, \\ Khoon Lim and Sandra Kieser
}

\begin{abstract}
Spinal epidural haematomas (SEH) occur from extra-dural bleeding, most commonly after trauma, epidural anaesthesia or operative intervention. However, these also occur in arterio-vascular malformations or spontaneously without an obvious inciting event. Spontaneous spinal epidural haematomas (SSEH) are rare, with a quoted incidence of 0.1 cases per 100,000 population annually. However, these haematomas carry a significant risk of spinal cord or nerve root compression potentially resulting in permanent neurological dysfunction or death. This chapter reviews the presentation, diagnosis and treatment of SSEH in adults, pregnancy and children in order to provide clinicians with an understanding of their typical presentation, required investigations and treatment algorithms.
\end{abstract}

Keywords: epidural, haematoma, spine

\section{Introduction}

Spinal epidural haematomas (SEH) occur from extra-dural bleeding, most commonly after trauma, epidural anaesthesia or operative intervention [1, 2]. These haematomas are rare but carry a significant risk of spinal cord or nerve root compression potentially resulting in permanent neurological dysfunction and death [3, 4].

Spontaneous spinal epidural haematomas (SSEH) occur without an obvious inciting event, although they may be associated with vascular malformations of the spinal canal. These haematomas are rare with a quoted incidence of approximately 0.1 cases per 100,000 population annually [5-7]. When they occur they often mimic other conditions such as cerebro-vascular accidents $[8,9]$, which may make them a difficult condition to diagnose, resulting in a delayed diagnosis and potentially mismanagement with anticoagulation therapy $[9,7,10]$. However, the consequences of SSEH can be devastating with progressive neurological decline, permanent cord injury and even death $[3,11,12]$. Thus, early and accurate diagnosis and management is essential to preserve neurological function and optimise the chance of recovery [13].

The aims of treatment in SSEH are to restore normal neurological function with minimal risk to the patient, however the method of achieving this remains uncertain [13]. Surgical decompression, typically through a partial or complete laminectomy or laminoplasty, allows direct decompression of the neural elements and evacuation of the haematoma but carries both anaesthetic and surgical risk. Less invasive options include radiologically guided aspiration of the haematoma, however this also carries 
risks of neural injury and further bleeding, often without complete evacuation of the haematoma. Medical therapy with steroids and/or pro-coagulation therapy is aimed at reducing local swelling and preventing further bleeding but fails to remove the compressive effects of the haematoma. Similarly, embolisation of the bleeding vessel may prevent further bleeding, but fails to remove the haematoma. The treatment approach is therefore complex and depends on the patient's presentation. Three defined groups of patients where the management varies are adults, pregnant women and children.

\section{Adults}

\subsection{Demographics and risk factors}

SSEH can affect all adult age groups, but most reported cases are aged between 50 and 80 years [14]. There is no obvious gender association, although pregnancy is an independent risk factor [14]. In most adult patients no known risk factors are identified, but according to the most recent review $19 \%$ had hypertension, $14 \%$ had cardiovascular disease, $3 \%$ had bleeding disorders and $6 \%$ had diabetes or obesity [14]. Interestingly, bleeding disorders did not specifically affect a younger population, but as expected by their cumulative medical comorbidities, patients on anticoagulation therapy were generally older. The most common anticoagulants identified in adults with SSEH were Warfarin (43.9\%), Aspirin (36.8\%) or Clopidogrel (10.5\%).

\subsection{Clinical presentation}

At presentation, most adults (98\%) complain of back and radicular pain [14]. Most haematomas reported in the literature cause spinal canal compression with severe caudal motor (58\% Medical Research Council grade 0 ) and sensory dysfunction (70\% American Spinal Injury Association (ASIA) A-C) [14]. In contrast, relatively few patients presented with compression of a nerve root in the neuroforamen, and in these cases most patients (63\%) maintain normal or near normal motor (70\% MRC grade 4 or 5) and sensory function (63\%) [14].

Of concern, up to $15 \%$ of adults with SSEH are suspected of having an alternative diagnosis, half of which are thought to be affected by an ischaemic incident for which anticoagulation therapy is considered or administered. The most common diagnoses that SSEH mimic are cerebro-vascular accidents (CVA), myocardial infarctions, spinal tumours and disc prolapses. Thus, clinicians should be aware of this condition and its ability to mimic alternative diagnoses, prior to considering anticoagulation therapy.

\subsection{Investigations}

The diagnosis is easily confirmed on cross-sectional imaging, most commonly an MRI scan or CT myelogram. An MRI is preferential as it offers a clear localisation and cause of compression (Figure 1). In contrast to a CT which accurately localises compression but is less accurate in determining the underlying cause (Figure 2 ).

In adults most SSEH affect the cervico-thoracic region between C3 and T3. This is attributed to this region being the only area of the spinal canal with a compact continuous epidural venous network [15]. These haematomas can be large and affect up to 28 spinal levels, with the average number of levels affected being 5.4 vertebral levels. Furthermore, although rare, multi-site haematomas can occur, therefore whole spine imaging is advocated. 


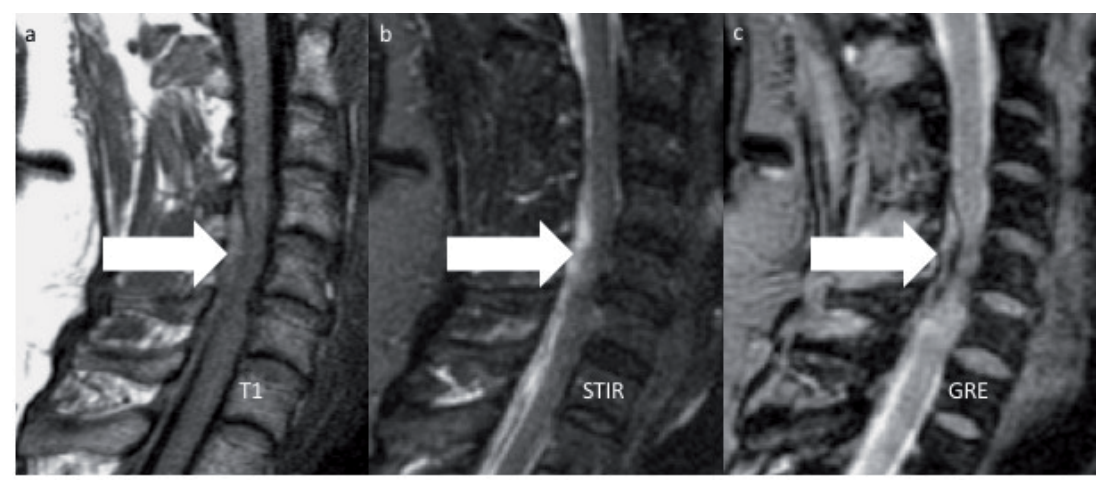

Figure 1.

A T1 (a), STIR (b) and GRE (c) sagittal MRI sequence of a C4-6 SSEH compressing the spinal cord.

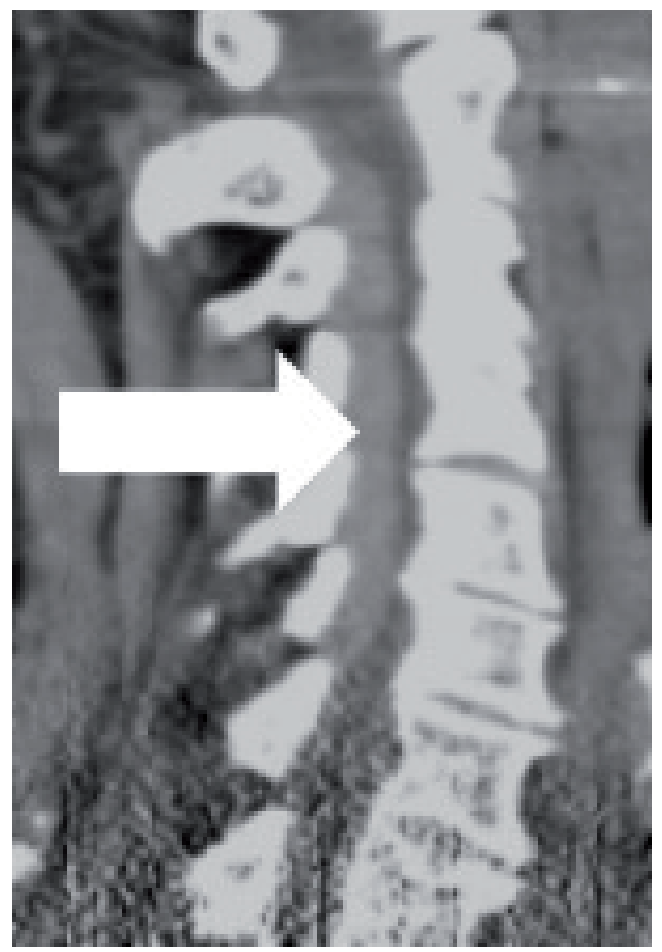

Figure 2.

A sagittal non-contrast CT scan of a C2-6 SSEH compressing the spinal cord.

\subsection{Treatment}

The gold standard of treatment for patients with SSEH and neurological dysfunction is expeditious surgical decompression. However, in patients with no neurology or with improving neurology, non-operative treatment with close neurological monitoring is the treatment of choice. There is no evidence to support operative intervention based on the presence of back pain or the size of the haematoma, rather, neurological dysfunction guides the decision to operatively intervene. In patients with a bleeding tendency, systemic pro-coagulant therapy should also be used.

With this approach to treatment, $85 \%$ of patients can be expected to make a complete or partial recovery [14]. This recovery is usually rapid, but in some patients this may take a number of years, which results in a reported average time to 
recovery of 52.4 days [14]. These outcomes are unrelated to patient age, sex, haematoma size or location.

According to the current literature, $5 \%$ of patients worsen their neurological function despite treatment and the mortality rate is 3.5\% [14]. The most common cause of death is cardiac disease rather than neurological dysfunction from SSEH.

\section{Pregnancy}

Unlike in the general population, SSEH carry the additional concern for the well-being of the foetus. Thus, the goal of treatment is to restore normal neurological function to the mother, but with minimal risk to the foetus [13].

Like the general adult population, the treatment options include surgical decompression, but this carries surgical and anaesthetic risks; radiological aspiration with or without embolisation, but this too carries anaesthetic risks, rarely evacuates the haematoma completely, risks neuro-vascular injury and exposes the mother and foetus to radiation; or chemotherapy with steroids and/or coagulation therapy, but this fails to remove the compressive effects of the haematoma and requires systemic administration of the drug. Thus, the treatment approach is complex and requires consideration of both the mother and foetus.

\subsection{Demographics and risk factors}

In pregnancy, an increased blood volume and pressure predisposes to rupture of vascular walls and therefore SSEH [16]. Thus, it is rare for SSEH to occur in the first trimester but the risk increases with the duration of pregnancy with an average gestational age of 34.3 weeks being reported in the literature [17]. No cases have been reported during labour, although some have been reported in the days and weeks following delivery, which suggests that the pregnancy-induced dilation of epidural vessels, rather than the mechanical straining of labour, is the predominant cause of SSEH in pregnancy.

There does not appear to be a relationship between the gravid status and the risk of SSEH [17]. Furthermore, most patients do not have any known risk factors, although pre-eclampsia has been reported in two cases. No pregnant patients with SSEH have been reported to be on anticoagulation therapy [17].

\subsection{Clinical presentation}

Like the general population, patients present with back or neck pain, usually with neurological dysfunction [17]. Within the English literature, most patients have severe spinal cord dysfunction with gait disturbance, caudal weakness and sensory disturbance. Only one reported patient has presented without neurological dysfunction [17].

\subsection{Investigations}

In pregnancy, limited radiation exposure to the foetus is necessary, thus an MRI is the investigation of choice. No patients have been reported to have had a CT scan, although, if an MRI is not possible and the diagnosis is critical then a CT or CT myelogram is necessary with protection of the pelvis [7].

Like the general adult population, the typical location of the SSEH is the cervico-thoracic region [17]. The size of the haematoma has been reported to span two to six spinal levels [17]. 


\subsection{Treatment}

In mothers with neurological dysfunction and less than 32 weeks of gestation, surgical decompression with foetal monitoring is advocated. In contrast, in mothers with neurological dysfunction and more than 32 weeks of gestation, a caesarean section followed by surgical decompression is advocated. Like in the general population, neurological dysfunction guides surgical intervention, not the presence of back pain or the size of the haematoma. Thus, in mothers without neurological dysfunction, close monitoring without surgical intervention is appropriate [17]. With such treatment most mothers are expected to make a complete (56\%) or partial (38\%) recovery.

\section{Children}

SSEH can occur in children. However, in contrast to adults the difficulty conversing with children and attaining investigations, such as cross-sectional imaging, makes the diagnosis difficult. In addition, their rarity and occasional atypical presentations mimicking alternative diagnoses compounds the challenges of diagnosis $[9,8]$. Yet, the consequences of this condition can be devastating.

\subsection{Demographics and risk factors}

Children of all ages (range 3 months - 18 years) have been identified with SSEH [18]. There does not appear to be a gender relationship to the risk of developing SSEH, however bleeding tendencies, such as haemophilia, which do construe a risk ( $8 \%$ of reported cases), are clearly more common in males [18]. One case was reported to be on anti-coagulation therapy and two have been reported to have an arterio-venous malformation.

\subsection{Clinical presentation}

Due to the variance in patient age, young children cannot report the location of pain, however, in those that are conversant most complain of back or neck pain, with radicular pain being less common [18]. Similarly, the neurological dysfunction, particularly sensory disturbance, is harder to define than in an adult population. However, most children are affected by neurological dysfunction (97\%), most commonly spinal cord dysfunction, although isolated nerve root dysfunction is also reported [18].

Nearly one third of all reported cases are initially suspected of having an alternative diagnosis, most commonly Guillain-Barre syndrome, meningitis, cerebral palsy, Grisel's syndrome or an acute abdomen [18].

\subsection{Investigations}

A whole spine MRI scan is the investigation of choice. A CT myelogram or noncontrast CT may be required depending on the patient's compliance and compatibility with an MRI scan, although the ionising radiation risk should be recognised and exposure limited.

As with adults the typical location of these haematomas are the cervico-thoracic region, and the size of the haematoma may span one to 22 spinal levels [18]. 


\subsection{Treatment}

Similar to adults, neurological dysfunction determines surgical intervention, not the presence of back pain or the size of the haematoma. Thus, in patients with neurological dysfunction, the gold standard treatment is surgical decompression and evacuation of the haematoma. The surgical approach to achieve this depends on the haematoma size, location, consistency and surgeon familiarity. While partial or complete laminectomies as well as laminoplasties have been reported, the consequences of each approach on the long-term consequences on spinal growth and function in these patients remain unknown. Thus, the least invasive approach to achieve decompression and haematoma evacuation should be utilised, but patients should also be counselled of the longer-term risks that surgical intervention may impose.

In patients without neurological dysfunction or with improving neurology, non-operative care with close neurological monitoring is appropriate. In patients with a bleeding tendency, systemic pro-coagulant therapy should be initiated, and if neurological improvement rapidly occurs, then non-operative treatment should be continued, but if no improvement occurs, then surgical intervention should be undertaken [18].

With this approach most children (83\%) are expected to make a complete (69\%) or partial (14\%) neurological recovery [18]. However, this condition can result in death, with two children having been reported to have died following a SSEH. One child died from respiratory failure after operative intervention and the other without a cause for death being defined, but with evidence of progressive neurological dysfunction after surgical decompression $[3,4]$.

\section{Vascular malformations}

While most SSEH are believed to occur in the absence of local vascular malformations, there remains debate as to the number of these haematomas that have a predisposing vascular pathology, most notably arterio-venous fistulas, malformations and aneurysms. This is predominantly due to a poor specificity of initial imaging modalities to identify small vascular pathologies in the presence of a epidural haematoma, the rarity of sending pathological specimens of the local vasculature for histological assessment and the lack of follow-up imaging. However, local vascular pathologies have been identified in spinal epidural haematomas that develop without an inciting event in all age groups, although it is more commonly reported in children [18].

Clearly, an area of vascular fragility, due to an underlying malformation can predispose to spontaneous bleeding. The location of the subsequent haematoma from the bleed depends on the location of the vascular disruption. In the case of an epidural haematoma, the pressure exerted on the neural structures may cause neurological dysfunction for which the treatment is the same as that of a SSEH. If surgical intervention is necessary then coagulation of the vascular anomaly at the time of surgery to prevent further bleeding is necessary. This is often inadvertently performed during the coagulation manoeuvres undertaken to stop bleeding at the time of surgery. Fortuitously, this typically treats the underlying vascular pathology and avoids recurrence.

\section{Summary}

To date, the major limitations of the literature reporting on SSEH are the low numbers, publication bias, the variance in imaging and reporting of vascular 
malformations and the lack of randomised control trials assessing outcome. However, it is clear that all age groups can be affected by SSEH and that three subsets of patients exist, namely adults, pregnant women and children.

In all groups, SSEH can present with variable symptoms and signs but most have neck or back pain with neurological dysfunction. In adults, SSEHs can mimic ischaemic events and lead to inappropriate anticoagulation therapy, which can be avoided by clinical awareness of this condition and whole spine cross-sectional imaging. Similarly, in children the diagnosis can be challenging due to the problems conversing with children and the variable presentations, often mimicking neuromuscular disorders. It is further compounded by the challenges of attaining crosssectional imaging, which is again diagnostic. In pregnancy, the further difficulties lie in the protection of the unborn foetus, with the avoidance of ionising radiation, but cross-sectional imaging remains diagnostic.

In all groups, most SSEH affect the cervico-thoracic region and span multiple spinal segments. The gold standard of treatment for patients with neurological dysfunction is surgical decompression and haematoma evacuation. However, in patients without neurological dysfunction, close neurological monitoring without surgical intervention is appropriate. In adults with bleeding disorders, procoagulant therapy should be used as an adjunct to treatment, however in children with bleeding disorders pro-coagulant therapy may be trialled prior to surgical intervention. In pregnancy, surgical intervention involves surgical decompression with foetal monitoring in those of gestational age less than 32 weeks and a caesarean section followed by decompression in those of gestational age greater than 32 weeks. In children, the long-term effect of surgical intervention on spinal growth and function should be recognised.

\section{Author details}

David Kieser ${ }^{1 *}$, Scheherezade Soltani ${ }^{2}$, Michael Wyatt ${ }^{1}$, Khoon Lim ${ }^{1}$ and Sandra Kieser ${ }^{1}$

1 School of Medicine, Department of Orthopaedics and Musculoskeletal Medicine, University of Otago, Christchurch, Christchurch, New Zealand

2 Division of Spinal Surgery, Oxford University NHS Foundation Trust, Oxford, England

*Address all correspondence to: kieserdavid@gmail.com

IntechOpen

(C) 2019 The Author(s). Licensee IntechOpen. This chapter is distributed under the terms of the Creative Commons Attribution License (http://creativecommons.org/licenses/ by/3.0), which permits unrestricted use, distribution, and reproduction in any medium, provided the original work is properly cited. (cc) BY 


\section{References}

[1] Wulf H. Epidural anaesthesia and spinal haematoma. Canadian Journal of Anaesthesia. 1996;43(12):1260-1271

[2] Awad JN, Kebaish KM, Donigan J, et al. Analysis of the risk factors for the development of post-operative spinal epidural haematoma. Journal of Bone and Joint Surgery. British Volume (London). 2005;87(9):1248-1252

[3] Hejazi N, Thaper PY, Hassler W. Nine cases of nontraumatic spinal epidural hematoma. Neurologia MedicoChirurgica (Tokyo). 1998;38(11):718-723

[4] Endo T, Suzuki S, Inoue T, et al. Prediction of neurological recovery in spontaneous spinal epidural hematoma using apparent diffusion coefficient values. Spinal Cord. 2014;52(10):729-733

[5] Aksay E, Kiyan S, Yuruktumen A, et al. A rare diagnosis in emergency department: Spontaneous spinal epidural hematoma. The American Journal of Emergency Medicine. 2008;26(7):835 e833-e835

[6] Huh J, Kwak HY, Chung YN, et al. Acute cervical spontaneous spinal epidural hematoma presenting with minimal neurological deficits: A case report. Anesthesiology and Pain Medicine. 2016;6(5):e40067

[7] Shima H, Yasuda M, Nomura M, et al. A spinal epidural hematoma with symptoms mimicking cerebral stroke. Nagoya Journal of Medical Science. 2012;74(1-2):207-210

[8] Deger SM, Emmez H, Bahadirli K, et al. A spontaneous spinal epidural hematoma in a hemodialysis patient: A rare entity. Internal Medicine. 2009;48(24):2115-2118

[9] Liou KC, Chen LA, Lin YJ. Cervical spinal epidural hematoma mimics acute ischemic stroke. The American Journal of Emergency Medicine. 2012;30(7):1321-1323

[10] Hsieh CF, Tung CS, Shih PY, et al. Cerebral hemosiderosis as a causative factor of vascular parkinsonism. Acta Neurologica Taiwanica. 2005;14(4):201-207

[11] Aristedis R, Dimitrios B. Spinal epidural hematoma mimicking subarachnoid hemorrhage: A case study. Surgical Neurology International. 2017;8:182

[12] Duffill J, Sparrow OC, Millar J, et al. Can spontaneous spinal epidural haematoma be managed safely without operation? A report of four cases. Journal of Neurology, Neurosurgery, and Psychiatry. 2000;69(6):816-819

[13] Xian H, Xu LW, Li CH, et al. Spontaneous spinal epidural hematomas: One case report and rehabilitation outcome. Medicine (Baltimore). 2017;96(44):8473

[14] Soltani S, Nogaro MC, Haleem S, Rupani N, Pyrovolou N, Kieser DC. Spontaneous spinal epidural haematomas in adults: A systematic review. JSNS. 2018;7:4

[15] Groen RJ, Begeer JH, Wilmink JT, et al. Acute cerebellar ataxia in a child with transient pontine lesions demonstrated by MRI. Neuropediatrics. 1991;22(4):225-227

[16] Yonekawa Y, Mehdorn HM, Nishikawa M. Spontaneous spinal epidural hematoma during pregnancy. Surgical Neurology. 1975;3(6):327-328

[17] Soltani S, Nogaro MC, Kieser SCJ, Wyatt MC, Kieser DC. Spontaneous spinal epidural haematomas in pregnancy-A systematic review. World Neurosurgery. (epub ahead of print) 
Spontaneous Spinal Epidural Haematomas

DOI: http://dx.doi.org/10.5772/intechopen.89175

[18] Soltani S, Nogaro MC,

Rougelot C, Newell N, Lim K,

Kieser DC. Spontaneous spinal epidural

haematomas in children. European

Spine Journal. (epub ahead of print) 


\section{Edited by Bora Gürer and Pinar Kuru Bektaşoğlu}

Vascular malformations of the central nervous system are important pathologies that could present with abrupt onset hemorrhage resulting in devastating neurological deficits. Current knowledge of their biology and natural history is increasing.

Diagnostic modalities help clinicians to better evaluate the individual cases, and to decide the best treatment options. Treatment alternatives are various and all treatment options should be evaluated before choosing the final therapeutic modality. The purpose of this book is to review the current knowledge about vascular malformations of the central nervous system and to evaluate the treatment alternatives.

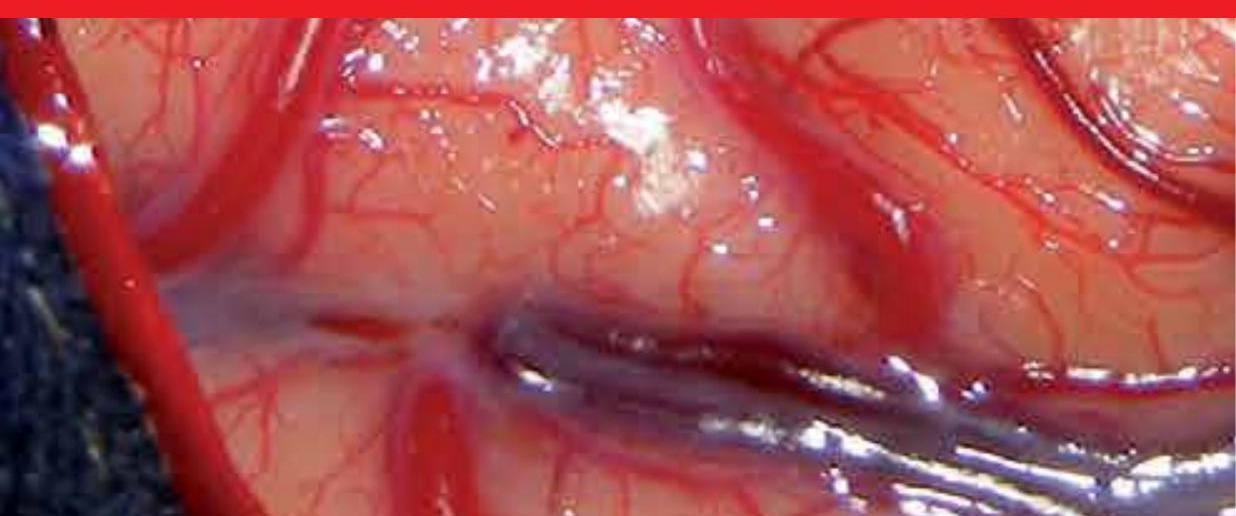

